\title{
Parametric Modeling and Optimization of
}

\section{Electromagnetic and Multiphysics Behaviors of \\ Microwave Components}

by

Wei Zhang, B.Eng

A thesis submitted to the Faculty of Graduate and Postdoctoral Affairs in partial fulfillment of the requirements for the degree of

\section{Doctor of Philosophy}

Electromagnetic Field and Microwave Technology, School of Microelectronics Tianjin University, Tianjin, China

Ottawa-Carleton Institute for Electrical and Computer Engineering Carleton University, Ottawa, Ontario, Canada

(C) 2019

Wei Zhang 


\begin{abstract}
Parametric modeling and optimization of electromagnetic (EM) and multiphysics behaviors are very essential parts of the design process of microwave components. For EM/multiphysics design, the computational cost of directly using EM/multiphysics simulator is very expensive because the simulation-driven design requires repetitive $\mathrm{EM} /$ multiphysics evaluations due to the adjustments of the values of design parameters. This thesis proposes new techniques to speed up the parametric modeling and design optimization of EM and multiphysics behaviors.

In the first part of this thesis, a novel technique is proposed to develop a lowcost EM centric multiphysics parametric model for microwave components. In the proposed method, we use space mapping (SM) techniques to combine the computational efficiency of EM single physics (EM only) simulation with the accuracy of the multiphysics simulation. The proposed model includes the EM domain coarse model and two mapping neural networks to map the EM domain (single physics) to the multiphysics domain. Our proposed technique can achieve good accuracy for multiphysics parametric modeling with fewer multiphysics training data and less computational cost.
\end{abstract}

As a further advancement, to accelerate the multiphysics design circle, we develop a novel parallel EM centric multiphysics optimization technique. The poleresidue-based transfer function is exploited to build an effective and robust surrogate model. A group of modified quadratic mapping functions is formulated to map the relationships between pole/residues of the transfer function and the design variables. Multiple EM centric multiphysics evaluations are performed in parallel 
to generate the training samples for establishing the surrogate model. Using our proposed technique, the surrogate model can be valid in a relatively large neighborhood which makes an effective and large optimization update in each optimization iteration. The trust region algorithm is performed to guarantee the convergence of the proposed multiphysics optimization algorithm. Our proposed multiphysics optimization technique takes a small number of iterations to obtain the optimal EM centric multiphysics response.

For the design of microwave components with various specifications, the geometrical parameters need to be explored in a large range. In the third part of this thesis, a novel decomposition technique is proposed to address the challenges of EM parametric modeling with geometrical changes in a large range. A systematic and automatic algorithm based on second-order derivative information is proposed to decompose the modeling problem with a wide geometrical range into a set of subrange modeling problems. Multiple artificial neural network (ANN) models, hereby referred to as sub-models are independently developed with geometrical parameters as variables in their own sub-ranges. A new technique is proposed to combine the developed sub-models to obtain a continuous overall model by solving the multidimensional discontinuity problem. The proposed technique provides an efficient mathematical methodology to perform the decomposition so that the cumbersome process can be done systematically and automatically. Compared with standard modeling methods using one model to cover the entire wide geometrical range, the proposed method can obtain better model-accuracy with short model-development time. The proposed parametric modeling and optimization techniques in this thesis 
are illustrated by several microwave examples. 
To my parents

Chunxiang $X u$ and Guili Zhang

To my wife

Kaige Qu 


\section{Acknowledgements}

I would like to express my sincere appreciation to my supervisor, Prof. Qi-Jun Zhang for his professional guidance, constant support, invaluable inspiration, and suggestions throughout the research work and preparation of this thesis. His encouragement, motivation and thoughtful insights during my research made my journey in realizing my goal a memorable one. It was my honor to work under his supervision and guidance.

I would also like to express my appreciation to my co-supervisor, Prof. Jianguo Ma for the encouragement and knowledgeable instruction. His professional suggestions provided a great help for me on my way pursuing my $\mathrm{Ph} . \mathrm{D}$ degree. I would like to thank Tianjin University, where I have stayed for six years accomplishing my Ph.D studies. I would also like to thank Carleton University for the Cotutelle program for my Ph.D study.

I would like to thank all my present and former colleagues especially Dr. Lin Zhu, Dr. Shuxia Yan, Dr. Venu-Madhav-Reddy Gongal-Reddy, Dr. Feng Feng, Dr. Chao Zhang, Dr. Weicong Na, Dr. Wenyuan Liu, Chuan Zhang, Shunlu Zhang, Kai Ma, Bo Peng, Jianan Zhang, Zhihao Zhao and Jing Jin in our research group for their enthusiasm and valuable discussions.

Many thanks to Blazenka Power, Anna Lee, Valerie Daley, Scott Bruce, Stephen Maclaurin, and other staff in the Department of Electronics, Carleton University, for offering a helpful and friendly yet professional support. Many thanks to Jia Liu, Shan Zhang and other staff in the Department of Microelectronics, Tianjin University, for all the support and suggestions. 
I would like to thank my friends Zhimin Jiang, Xiaolin Qiu, Hui Zong, Jiarong Zhang, Shuonan Hou, Xiao Jin, Wen Wen, Junhao Chen, Ruixue Wang, Ao Zhang, and Zhaopeng Wang for the helps and memorable experiences during my Ph.D period.

Finally, I wish to thank my parents, my father-in-law, my mother-in-law, my uncles and aunts for their love, belief, support and encouragement. A special thank to my dear wife Kaige Qu for her constant accompany, belief, encouragement, understanding and love throughout my life. 


\section{Table of Contents}

Abstract $\quad$ i

Acknowledgements $\quad$ V

List of Figures $\quad$ xii

List of Tables $\quad$ xxix

1 Introduction 1

1.1 Background and Motivation . . . . . . . . . . . . . . 1

1.2 List of Contributions . . . . . . . . . . . . . . . . 5

1.3 Thesis Organization . . . . . . . . . . . . . . . 7

2 Literature Review $\quad 10$

2.1 Artificial Neural Network . . . . . . . . . . . . . . . . . . 10

2.1.1 Neural Network Structures . . . . . . . . . . . . . . . . . 12

2.1.2 Neural Network Training . . . . . . . . . . . . . 15

2.2 Knowledge-Based Neural Network . . . . . . . . . . . . . . . 17 
2.3 Combined Neural Networks and Transfer Function . . . . . . . . . . 19

2.4 Space Mapping . . . . . . . . . . . . . . . . . . . . 22

2.4.1 Space Mapping Concept . . . . . . . . . . . . . . . . 24

2.4.2 Input Space Mapping . . . . . . . . . . . . . . . . . . 25

2.4.3 Implicit Space Mapping . . . . . . . . . . . . . . 26

2.4.4 Output Space Mapping . . . . . . . . . . . . . 27

2.4.5 Tuning Space Mapping . . . . . . . . . . . . . . 28

2.4.6 Neuro Space Mapping . . . . . . . . . . . . . . . . . . 29

2.4.7 Parallel Space Mapping _. . . . . . . . . . . . . . . . . 29

2.5 EM Centric Multiphysics Simulations . . . . . . . . . . . . . 30

2.5.1 Description of the EM centric Multiphysics Problem for Microwave Components . . . . . . . . . . . . . . 32

2.6 Decomposition Techniques . . . . . . . . . . . . . . . . 34

2.7 Conclusion . . . . . . . . . . . . . . . . . . . . 34

\section{Space Mapping Approach to Electromagnetic Centric Multiphysics} Parametric Modeling of Microwave Components 36

3.1 Introduction . . . . . . . . . . . . . . . . . . 37

3.2 Proposed EM centric Multiphysics Parametric Modeling Technique 39

3.2.1 Structure of the Proposed Space Mapped EM Centric Multiphysics Parametric Model . . . . . . . . . . . . . . . . . 39

3.2.2 EM Domain Coarse Model Construction . . . . . . . . . . 46 
3.2.3 Proposed Space Mapped EM Centric Multiphysics Parametric Model Training Process . . . . . . . . . . . . . 47

3.2.4 Use of Proposed Model for Multiphysics Design Optimization 53

3.3 Numerical Examples . . . . . . . . . . . . . . . . . . 56

3.3.1 Multiphysics Parametric Modeling of Tunable Four-Pole Waveguide Filter Using Piezo Actuator . . . . . . . . . . . . . 56

3.3.2 Multiphysics Parametric Modeling of an Iris Coupled Microwave Cavity Filter . . . . . . . . . . . . . . . . . . 72

3.4 Conclusion ........................... 82

\section{EM Centric Multiphysics Optimization of Microwave Components} Using Parallel Computational Approach $\quad 85$

4.1 Introduction . . . . . . . . . . . . . . . . 86

4.2 Parallel EM Centric Multiphysics Optimization . . . . . . . . . 87

4.2.1 Parallel EM Centric Multiphysics Evaluation . . . . . . . . . 88

4.2.2 Proposed Surrogate Model for EM Centric Multiphysics Optimization ....................... 90

4.2.3 Proposed Parallel EM Centric Multiphysics Optimization . . 96

4.3 Numerical Examples . . . . . . . . . . . . . . . . . . . . . 102

4.3.1 Multiphysics Optimization of Tunable Evanescent Mode Cavity Filter . . . . . . . . . . . . . . . . . . . 102

4.3.2 Multiphysics Optimization of Tunable Four-Pole Waveguide Filter Using Piezo Actuator . . . . . . . . . . . . . 107 
4.4 Conclusion . . . . . . . . . . . . . . . . . . . . . . . . 115

5 Parallel Decomposition Approach for Wide Range Parametric Mod$\begin{array}{ll}\text { eling of Microwave Components } & 117\end{array}$

5.1 Introduction . . . . . . . . . . . . . . . . . 118

5.2 Proposed Decomposition Technique for Wide Range Parametric Mod-

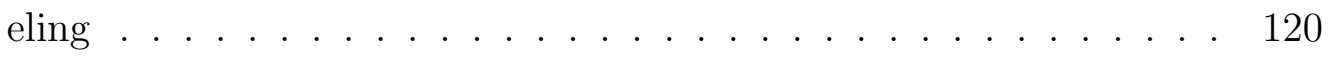

5.2.1 Description of Wide Range Problem . . . . . . . . . . . 120

5.2.2 Formulation of Second-Order Derivative . . . . . . . . . 122

5.2.3 Proposed Decomposition Technique Using Second-Order Derivative . . . . . . . . . . . . . . 126

5.2.4 Parallel Sub-Model Training Process _. . . . . . . . . . 135

5.3 Proposed Combination Technique for Wide Range Parametric Modeling 136

5.3.1 The Discontinuity Problem for Adjacent Sub-models . . . 137

5.3.2 Proposed Technique to Solve the Discontinuity Problem . . . 138

5.4 Numerical Examples . . . . . . . . . . . . . . . . . . . 147

5.4.1 Parametric Modeling of a Bandstop Microstrip Filter with Open Stubs Incorporating Decomposition Technique . . . 147

5.4.2 Parametric Modeling of an Inter-Digital Bandpass Filter Incorporating Decomposition Technique . . . . . . . . . 158

5.4.3 Parametric Modeling of a Four-Pole Waveguide Filter Incorporating Decomposition Technique . . . . . . . . . . 169

5.5 Conclusion . . . . . . . . . . . . . . . 180 
6 Conclusions and Future Research 182

6.1 Conclusions ............................ 182

6.2 Future Research . . . . . . . . . . . . . . . . . . . 184

Bibliography

195 


\section{List of Figures}

2.1 The multilayer perceptron (MLP) structure cosisting of one input layer, one or more hidden layers, and one output layer. . . . . . . . 13

2.2 A recurrent neural network structure with feedback of delayed neural network output. . . . . . . . . . . . . . . . . 15

2.3 Illustration of the structure of knowledge-based neural network (KBNN)

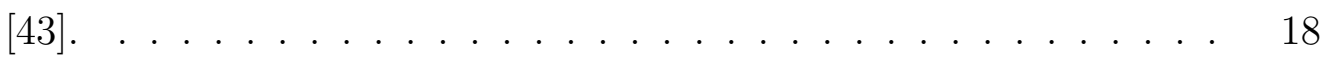

2.4 The structure of the pole-residue-based neuro-TF model. $\boldsymbol{x}$ represents the geometrical variables. $\boldsymbol{y}$ represents real and imaginary parts of the outputs of the pole-residue-based transfer function (e.g., S-parameters). $\boldsymbol{d}$ represents the outputs of the EM simulations. . . 20

2.5 The illustration of space mapping concept. . . . . . . . . . 23

2.6 The mathematical representation of the space mapping methodology. 25

2.7 The mathematical representation of the implicit space mapping methodology. . . . . . . . . . . . . . . . . . . 27 27

2.8 The illustration of neuro space mapping concept . . . . . . . . 30 
2.9 The iterative process of multiphysics analysis of the microwave structure. This multiphysics analysis includes three physics domains, EM, thermal, and structure mechanical. . . . . . . . . . . . 32

3.1 Simple illustration of the idea of using space mapping techniques to build the mapping relationship between the multiphysics domain and single physics domain. (a) Original structure of a film capacitor, (b) Structure for electric analysis, i.e., the coarse model, (c) Deformed structure due to high power at one side of the capacitor used for multiphysics analysis, i.e., the fine model and (d) Electric analysis with a different width such that the capacitance of this non-deformed structure (i.e., the mapped coarse model) is the same as the capacitance of deformed structure shown in (c). $W$ is changed from $12 \mathrm{~mm}$ to 15 mm by mapping. . . . . . . . . . . . . . . . . . . . . . . . . . . . . 40 
3.2 Structure of the proposed space mapped multiphysics parametric model exploiting coarse model and space mapping techniques. $\boldsymbol{R}_{s}$ represents the real and imaginary parts of the outputs of the overall multiphysics model (e.g., S-parameters); $\boldsymbol{R}_{f}$ represents the outputs of fine model multiphysics analysis. The first mapping module represents the relationship between the multiphysics domain design parameters and EM domain design parameters. The second mapping module represents space mapping between the non-geometrical input parameters along with frequency parameter of the multiphysics model and the frequency parameter of the EM domain coarse model.

3.3 Structure of the proposed adjoint multiphysics model including the adjoint EM domain coarse model and two adjoint neural network models. The adjoint model of the first mapping module is the adjoint neural network of $f_{A N N 1}$ and the adjoint model of the second mapping module is the adjoint neural network of $f_{A N N 2}$. The purpose of this adjoint model is to privide the derivative information to guide the training and optimization process. . . . . . . . . . . . .

3.4 The detailed training mechanism of the overall multiphysics parametric model exploiting space mapping technique. The objective is to minimize the training error between the proposed model and multiphysics data. The variables of this training process are the weighting parameters $\boldsymbol{w}_{1}$ and $\boldsymbol{w}_{2}$ of the two mapping modules between the multiphysics domain and the single physics domain. . . . . . . . . 
3.5 The flowchart of the development process of the multiphysics parametric model exploiting the EM domain coarse model and space mapping between the multiphysics domain and the single physics EM domain. ......................... 54

3.6 Structure of the four-pole waveguide filter using piezo actuator with multiphysics model design variables $\boldsymbol{x}=\left[\begin{array}{llllll}h_{1} & h_{2} & h_{c 1} & h_{c 2} & V_{1} & V_{2}\end{array}\right]^{T}$. The input and output waveguides, as well as the resonant cavities, are standard WR-75 waveguides (width $=19.050 \mathrm{~mm}$, height $=9.525$ $\mathrm{mm})$. The length of the structure is $77.6 \mathrm{~mm}$. The thickness of all the coupling windows is set to $2 \mathrm{~mm} . \quad \ldots . . . . . .557$

3.7 The actual process of the multiphysics problem for the four-pole waveguide filter example using the COMSOL software. . . . . . .

3.8 The structural deformation in the four-pole waveguide filter caused by the input voltages. . . . . . . . . . . . . . . .

3.9 Comparison of the magnitude in decibels of $S_{11}$ of the EM single physics (EM only) responses and multiphysics analysis responses using the same geometrical parameters for the four-pole waveguide filter. From the figure we can see that without mapping, the single physics analysis is not accurate enough to represent the multiphysics responses. . . . . . . . . . . . . . . . . 
3.10 Comparison of the magnitude in decibels of $S_{11}$ of the overall multiphysics models developed using different modeling methods and COMSOL MULTIPHYSICS data: (a) Test sample \#1 and (b) Test sample \#2 for the four-pole waveguide example. In the figure, NeuroTF model (less data) means the Neuro-TF model with correlating mapping trained with less multiphysics data. Neuro-TF model (more data) means the Neuro-TF model with correlating mapping trained with more multiphysics data. Proposed model (less data) means the proposed model trained with less multiphysics data. . . . . . . . .

3.11 The proposed parametric model is used for optimization w.r.t. two separate cavity filters with two different specifications for the fourpole waveguide filter. The optimal solution is found by our model and verified by the COMSOL MULTIPHYSICS. The magnitude in decibels of $S_{11}$ and $S_{21}$ of COMSOL MULTIPHYSICS data at (a) Optimized design solution for filter 1 and (b) Optimized design solution for filter 2. As shown in the figure, the proposed model behaves well in design optimization with different specifications. . . . . . . . 
3.12 The proposed parametric model is used for filter tuning w.r.t. two different specifications for the four-pole waveguide filter. The optimal tuning solution is found by our model and verified by the COMSOL MULTIPHYSICS. The magnitude in decibels of $S_{11}$ of COMSOL MULTIPHYSICS data at (a) Initial point: $V_{1}=0 \mathrm{~V}$ and $V_{2}=0 \mathrm{~V}$, (b) Optimized tuning solution for specification 1: $V_{1}=230.429 \mathrm{~V}$ and $V_{2}=233.428 \mathrm{~V}$ and (c) Optimized tuning solution for specification 2: $V_{1}=-244.4 \mathrm{~V}$ and $V_{2}=-236.589 \mathrm{~V}$. As shown in the figure, the proposed model behaves well in tuning optimization with different specifications. . . . . . . . . . . . . . . .

3.13 Extrapolation results of the test sample slightly beyond the training ranges for the four-pole waveguide filter example. We can see that the approximate results can be obtained by our proposed model. . .

3.14 Frequency extrapolation results of the test sample in the frequency range from $9.5 \mathrm{GHz}$ to $12 \mathrm{GHz}$ for the four-pole waveguide filter example. . . . . . . . . . . . . . . . . .

3.15 (a) Structure of the iris coupled waveguide filter where a high input power is supplied to port 1 . The design variables are $\boldsymbol{x}=$ $\left[\begin{array}{lllll}w_{1} & w_{2} & w_{3} & w_{4} & P_{i n}\end{array}\right]^{T}$, (b) Temperature distribution in the iris coupled waveguide filter caused by the large input power and (c) Structural deformation in the iris coupled waveguide filter caused by the temperature distribution. . . . . . . . . . . . . . . 
3.16 The actual process of the multiphysics simulation for the iris coupled waveguide filter example using the ANSYS WORKBENCH software.

3.17 Comparison of the magnitude in decibels of $S_{11}$ of the EM single physics (EM only) response and multiphysics analysis response using the same geometrical parameters for the iris coupled waveguide filter. From the figure we can see that without mapping, the single physics analysis is not accurate enough to represent the multiphysics responses. 76

3.18 Comparison of the magnitude in decibels of $S_{11}$ of the models developed using different modeling methods and ANSYS WORKBENCH data: (a) Test sample \#1 and (b) Test sample \#2 for the iris waveguide filter. In the figure, ANN model (less data) means the ANN model trained with less multiphysics data. ANN model (more data) means the ANN model trained with more multiphysics data. Proposed model (less data) means the proposed model trained with less multiphysics data. . . . . . . . . . . . . . . . . 83

4.1 Illustration of multiple samples used to train the surrogate model. The orthogonal distribution is used to generate the multiple samples $\left(\widehat{\boldsymbol{x}}_{1}^{k}, \widehat{\boldsymbol{x}}_{2}^{k}, \cdots, \widehat{\boldsymbol{x}}_{n_{s}}^{k}\right)$ around the central point $\boldsymbol{x}_{c}^{k}$. The central point $\boldsymbol{x}_{c}^{k}$ is updated after each iteration of the proposed optimization method. The updated central point $\boldsymbol{x}_{c}^{k+1}$ and the orthogonal samples around the new central point move as the proposed optimization progresses from the $k$ th iteration to the $(k+1)$ th iteration. . . . . . . . 
4.2 The structure of the proposed surrogate model with pole/residue based transfer function. The proposed model consists of a transfer function and $n_{3}+1$ quadratic functions, where $\boldsymbol{w}$ represents the weighting parameters in the quadratic functions; $\boldsymbol{x}$ represents the design parameters; $y_{s}$ represents the surrogate model outputs; $\widehat{y}_{m}$ represents the data from EM centric multiphysics simulation. . . . .

4.3 Flowchart of the proposed parallel EM centric multiphysics surrogate optimization process. . . . . . . . . . . . . . . 100

4.4 The tunable cavity filter structure for multiphysics optimization with four design parameters. . . . . . . . . . . . . . . 103

4.5 The practical process of the multiphysics analysis for the tunable evanescent mode cavity filter example. . . . . . . . . . . . . . . 103

4.6 The deformed structure of the tunable cavity filter example due to the piezo electric effects. . . . . . . . . . . . . . . . . . . . 104

4.7 The structure of the proposed surrogate model for the cavity filter example..................... 106

4.8 EM centric multiphysics responses for the tunable evanescent mode cavity filter example. (a) Multiphysics response at the starting point. (b) Multiphysics response using the proposed optimization techniques (after four iterations). The interval indicates the design specification for this cavity filter example. . . . . . . . . . . . . . . . . 107 
4.9 Objective function values of the tunable evanescent mode cavity filter example using the proposed multiphysics optimization method. The proposed optimization can converge in four iterations to reach the optimal solution. . . . . . . . . . . . . . . . . . . . . . 109

4.10 Four-pole waveguide filter structure using piezo actuator for multiphysics optimization with six design parameters. . . . . . . . . . 109

4.11 The practical process of the multiphysics analysis for the four-pole waveguide filter example. . . . . . . . . . . . . . . . 110

4.12 The deformed structure of the four pole waveguide filter due to the piezo electric effects. . . . . . . . . . . . . . . . . 11

4.13 The structure of the surrogate model for the tunable evanescent mode cavity filter example. . . . . . . . . . . . . . . .

4.14 EM centric multiphysics responses for the four-pole waveguide filter example. (a) Multiphysics response at the starting point. (b) Multiphysics response using the proposed optimization techniques (after five iterations). The interval indicates the design specification for this waveguide filter example. . . . . . . . . . . . . .

4.15 Objective function values of the four pole wavaguide filter example using the proposed multiphysics optimization method. The proposed optimization can converge in five iterations to reach the optimal so-

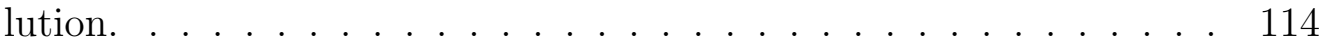


5.1 The proposed decomposition process to decompose the entire range $\boldsymbol{X}$ dimension by dimension for one training stage. The decomposition process starts from the first design variable $x_{1}$. When the last design variable $x_{N}$ is decomposed, we can automatically obtain the subranges for all the sub-regions. . . . . . . . . . . . . . . . . . . 134

5.2 (a) The four sub-models after the training process for the $2 \mathrm{D}$ example with four sub-ranges. (b) The sub-model responses along the diagonal line of $x_{1}$ and $x_{2}$ going through the sub-model 1 and sub-model 4 . The outputs of the adjacent sub-models at the boundary are different and the overall model is not continuous. . . . . . . . . . . . . . 138

5.3 The demonstration of the sigmoid function with different values of $a$ and $c$. We can observe the relationship between the parameter $a$ and the transition range $W \ldots \ldots \ldots$. . . . . . . . . . . . 140

5.4 The flowchart for calculating the total modification function of $\Psi^{t}$ for the $t^{t h}$ sub-model. . . . . . . . . . . . . . . . . . . . . 144

5.5 The flowchart for developing the proposed parametric model using decomposition technique. The proposed technique takes several training stages to obtain an accurate and continuous overall model. Each training stage includes performing the decomposition for all the geometrical parameters, training the ANN sub-models using parallel training, and combining the developed sub-models into overall model. 147 
5.6 (a) The overall model function after the proposed modification process for the 2D example with four sub-ranges. (b) The connection result along the diagonal line of $x_{1}$ and $x_{2}$ going through the submodel 1 and sub-model 4. After the modification process, a continuous model over the entire region is obtained. Our proposed technique can successfully solve the discontinuity problem. . . . . . . . . 148

5.7 The bandstop microstrip filter with quarter-wave resonant open stubs. The design variables for this example are $\boldsymbol{x}=\left[\begin{array}{ll}l_{0} & l_{1} l_{2}\end{array}\right] \ldots \ldots 149$

5.8 The sub-range for each sub-model after decomposition process at training stage two for the bandstop microstrip filter example. The design parameters for this example are $\boldsymbol{x}=\left[l_{0} l_{1} l_{2}\right]$. At the second training stage, the number of division $\boldsymbol{L}$ is calculated as [2 32 2]. At the first level decomposition, the range of geometrical parameter $l_{0}$ is decomposed into 2 sub-ranges. At the second level decomposition, the range of geometrical parameter $l_{1}$ is decomposed into 3 sub-ranges. At the third level decomposition, the range geometrical parameter $l_{2}$ is decomposed into 2 sub-ranges. The total number of sub-models is $n=2 \cdot 3 \cdot 2=12 \ldots \ldots \ldots \ldots \ldots \ldots \ldots \ldots \ldots \ldots$ 
5.9 Comparison of the magnitude of $S_{11}$ of the overall parametric model developed using different modeling methods and HFSS simulation data: (a) test sample \#1 and (b) test sample \#2 for the bandstop microstrip example. Our proposed model combining many sub-models is more accurate than standard ANN model using a single ANN for the entire wide range modeling because the proposed technique focuses on learning each small sub-region by a separate sub-model, while the standard modeling technique using a single ANN for the entire wide range modeling needs to compromise the ANN accuracy between different regions. . . . . . . . . . . . . . . . . . . 156

5.10 Demonstration of the continuity of the proposed overall model with design variables $\left[\begin{array}{lll}l_{0} & l_{1} & l_{2}\end{array}\right]$. The real and imaginary parts of $S_{11}$ (a) along the direction of $l_{2}$ going through sub-models $33,34,35$, and 36 ; (b) along the diagonal direction of $l_{1}$ and $l_{2}$ going through submodels 33, 37, 38, 42, 43, 47, and 48; and (c) along the diagonal direction of $l_{0}, l_{1}$, and $l_{2}$ going through sub-models $1,5,6,22,23,26$, 27, 43, 47, and 48. The proposed technique provides accurate and also continuous solutions along different directions. Our solutions are verified with HFSS simulation results. . . . . . . . . . . . . . 157 
5.11 The proposed parametric model is used for design optimization for the bandstop microstrip filter. The optimal solution is found by optimizing design variables $\left[\begin{array}{lll}l_{0} & l_{1} & l_{2}\end{array}\right]$ using our proposed model. The optimal solution is verified by HFSS simulation. The magnitude of $S_{11}$ of HFSS simulation data at (a) initial point in sub-model 23 and (b) optimal solution point in sub-model 1. Our proposed model behaves well in design optimization where the optimization moves freely and continuously between different sub-models. . . . . . . . . .

5.12 Structure of the inter-digital bandpass filter. The design variables for

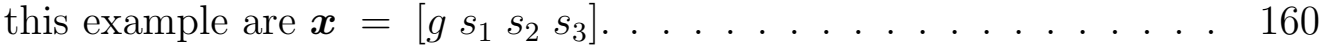

5.13 The sub-range for each sub-model after decomposition process at first training stage for the inter-digital bandpass filter example. The

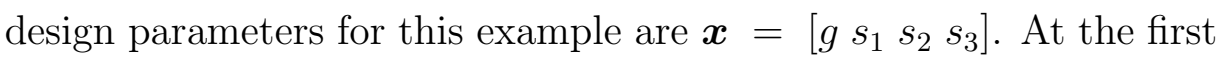
training stage, the number of division $\boldsymbol{L}$ is calculated as $\left[\begin{array}{lll}1 & 1 & 2\end{array}\right]$. This means only the geometrical parameters $s_{2}$ and $s_{3}$ are decomposed at this stage. At the first and second level decomposition, the entire wide range is not decomposed. At the third level decomposition, the range geometrical parameter $s_{2}$ is decomposed into 2 sub-ranges. At the fourth level decomposition, the range geometrical parameter $s_{3}$ is decomposed into 2 sub-ranges. The total number of sub-models is $n=1 \cdot 1 \cdot 2 \cdot 2=4 \ldots \ldots \ldots 162$ 
5.14 Comparison of the magnitude (in decibels) of $S_{11}$ of the overall parametric models developed using different modeling methods and HFSS simulation data: (a) test sample \#1 and (b) test sample \#2 for the inter-digital bandpass filter example. Our proposed model combining many sub-models is more accurate than standard ANN model using a single ANN for the entire wide range modeling because the proposed technique focuses on learning each small sub-region by a separate sub-model, while the standard modeling technique using a single ANN for the entire wide range modeling needs to compromise the ANN accuracy between different regions. . . . . . . . . . . . . 167

5.15 Demonstration of the continuity of the proposed overall model with design variables $\boldsymbol{x}=\left[\begin{array}{llll}g & s_{1} & s_{2} & s_{3}\end{array}\right]$. The real and imaginary parts of $S_{11}$ (a) along the direction of $g$ going through sub-models 11 and 59; (b) along the diagonal direction of $g$ and $s_{3}$ going through sub-models 10, 11, 12, 59, and 60; (c) along the diagonal direction of $g, s_{2}$, and $s_{3}$ going through sub-models $1,4,5,8,56$, and 60; and (d) along the diagonal direction of $g, s_{1}, s_{2}$, and $s_{3}$ going through sub-models 1, 4, $5,8,20,56,60$, and 72 . The proposed technique provides accurate and also continuous solutions along different directions. Our solutions are verified with HFSS simulation results. . . . . . . . . . . . 168 
5.16 The proposed parametric model is used for design optimization for the inter-digital bandpass filter. The optimal solution is found by optimizing design variables $\left[\begin{array}{llll}g & s_{1} & s_{2} & s_{3}\end{array}\right]$ using our proposed model. The optimal solution is verified by HFSS simulation. The magnitude (in decibels) of $S_{11}$ of HFSS simulation data at (a) initial point in submodel 2 and (b) optimal solution point in sub-model 8. Our proposed model behaves well in design optimization where the optimization moves freely and continuously between different sub-models. . . .

5.17 Structure of the four-pole waveguide filter. The design variables for

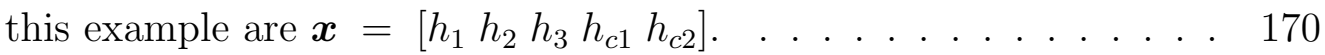

5.18 The sub-range for each sub-model after decomposition process at first training stage for the four-pole waveguide filter example. The design parameters for this example are $\boldsymbol{x}=\left[\begin{array}{lllll}h_{1} & h_{2} & h_{3} & h_{c 1} & h_{c 2}\end{array}\right]$. At the first training stage, the number of division $\boldsymbol{L}$ is calculated as $\left[\begin{array}{lllll}1 & 1 & 1 & 2 & 3\end{array}\right]$. This means only the geometrical parameters $h_{c 1}$ and $h_{c 2}$ are decomposed at this stage. At the first, second, and third level decomposition, the entire wide range is not decomposed. At the fourth level decomposition, the range geometrical parameter $h_{c 1}$ is decomposed into 2 sub-ranges. At the fifth level decomposition, the range geometrical parameter $h_{c 2}$ is decomposed into 3 sub-ranges. The total number of sub-models is $n=1 \cdot 1 \cdot 1 \cdot 2 \cdot 3=6$. . . . . . 173 
5.19 Comparison of the magnitude (in decibels) of $S_{11}$ of the overall parametric models developed using different modeling methods and HFSS simulation data: (a) test sample \#1 and (b) test sample \#2 for the four-pole waveguide filter example. Our proposed model combining many sub-models is more accurate than standard ANN model using a single ANN for the entire wide range modeling because the proposed technique focuses on learning each small sub-region by a separate sub-model, while the standard modeling technique using a single ANN for the entire wide range modeling needs to compromise the ANN accuracy between different regions. . . . . . . . . . . . . 177

5.20 Demonstration of the continuity of the proposed overall model with design variables $\boldsymbol{x}=\left[\begin{array}{lllll}h_{1} & h_{2} & h_{3} & h_{c 1} & h_{c 2}\end{array}\right]$. The real and imaginary parts of $S_{11}$ (a) along the direction of $h_{1}$ going through sub-models 52 and 112; (b) along the diagonal direction of $h_{1}$ and $h_{2}$ going through sub-models 23 and 112; (c) along the diagonal direction of $h_{1}, h_{2}$, and $h_{3}$ going through sub-models 7,97 , and $112 ;(d)$ along the diagonal direction of $h_{1}, h_{2}, h_{3}$, and $h_{c 1}$ going through sub-models $2,7,97$, 112, and 117; and (e) along the diagonal direction of $h_{1}, h_{2}, h_{3}$, $h_{c 1}$, and $h_{c 2}$ going through sub-models $1,7,97,113,118,119$, and 120. The proposed technique provides accurate and also continuous solutions along different directions. Our solutions are verified with HFSS simulation results. . . . . . . . . . . . . . . . . . 179 
5.21 The proposed parametric model is used for design optimization for the four-pole waveguide filter. The optimal solution is found by optimizing design variables $\left[h_{1} h_{2} h_{3} h_{c 1} h_{c 2}\right]$ using our proposed model. The optimal solution is verified by HFSS simulation. The magnitude (in decibels) of $S_{11}$ of HFSS simulation data at (a) initial point in sub-model 52 and (b) optimal solution point in sub-model 103. Our proposed model behaves well in design optimization where the optimization moves freely and continuously between different sub-models. 181 


\section{List of Tables}

3.1 Definition of Training and Testing Data for EM Domain (Single Physics) Coarse Model and Multiphysics Domain Overall Model for the Four-Pole Waveguide Filter Example . . . . . . . . . . . . . . . 61

3.2 Accuracy Comparisons of Different Methods for Parametric Modeling of the Four-Pole Waveguide Filter Example . . . . . . . . . . . . . . 64

3.3 CPU Comparisons of Different Methods for Parametric Modeling of the Four-Pole Waveguide Filter Example . . . . . . . . . . . . . . . 65

3.4 Comparison of Computation Time Between Multiphysics Non-parametric Simulation and Proposed Multiphysics Parametric Model of the FourPole Waveguide Filter Example . . . . . . . . . . . . . . . . . . . . 66

3.5 Definition of Training and Testing Data for EM Domain Coarse Model and Overall Multiphysics Fine Model For the Iris Coupled Waveguide Filter . . . . . . . . . . . . . . . . . . . . 77

3.6 Accuracy Comparisons of Different Methods for Parametric Modeling of the Iris Coupled Waveguide Filter _ . . . . . . . . . . . . 80

xxix 
3.7 CPU Comparisons of Different Methods for Parametric Modeling of the Iris Coupled Waveguide Filter . . . . . . . . . . . . . . . . . 81

3.8 Comparison of Computation Time Between Multiphysics Non-parametric Simulation and Proposed Multiphysics Parametric Model of the Iris Coupled Waveguide Filter . . . . . . . . . . . . . . . . . 82

4.1 Comparisons of Two Optimization Algorithms for the Tunable Evanescent Mode Cavity Filter Example . . . . . . . . . . . . . . . . . 108

4.2 Comparisons of Two Optimization Algorithms for the Four-Pole Waveguide Filter Example . . . . . . . . . . . . . . . . . . . . . 115

5.1 Comparisons of Different Methods for Parametric Modeling of the Bandstop Filter Example . . . . . . . . . . . . . . . . . 154

5.2 Comparisons of Different Methods for Parametric Modeling of the Inter-Digital Bandpass Example . . . . . . . . . . . . . . . 165

5.3 Comparisons of Different Methods for Parametric Modeling of the Four-Pole Waveguide Filter Example . . . . . . . . . . . . . 176 


\section{List of Symbols}

$a$

$a_{i}^{t, l}$

$a_{i}^{t, u}$

$\boldsymbol{A}^{k}$

$\boldsymbol{B}_{S M}$

$\boldsymbol{b}^{k}$
The coefficient of sigmoid function which controls the width of the transition range of the sigmoid function

The coefficient of sigmoid function for modification of sub-model $t$ at the lower boundary along the dimension of $x_{i}$, which controls the width of the transition range

The coefficient of sigmoid function for modification of sub-model $t$ at the upper boundary along the dimension of $x_{i}$, which controls the width of the transition range

A $N_{t} \times N_{u}$ matrix for calculating second order derivative of EM responses w.r.t. $k^{\text {th }}$ training sample

The scaling coefficients of a linear space mapping function

A $N_{t} \times 1$ vector for calculating second order derivative of EM responses w.r.t. $k^{\text {th }}$ training sample 
$C_{i}^{t}$

c

$c$

$c_{i}$

$c_{s}^{i,(l)}$

$\boldsymbol{c}_{S M}$

$c_{i}^{t, u}$

$c_{i}^{t, l}$

$D_{i}$

E

$E_{T e}$
The total value of the second-order derivative w.r.t. $x_{i}$ at all $N_{i}$ training samples in sub-region t

The coefficient of sigmoid function which controls the center point of the transition of the sigmoid function

A vector including all the poles and residues of the transfer function

One of the element in $\boldsymbol{c}$, which is also the output response of the modified quadratic mapping function

One of the quadratic functions

The shifting coefficients of a linear space mapping function

The coefficient of sigmoid function for modification of sub-model

$t$ at the lower boundary along the dimension of $x_{i}$, which controls the center point of the transition

The coefficient of sigmoid function for modification of sub-model $t$ at the upper boundary along the dimension of $x_{i}$, which controls the center point of the transition

The range of the geometrical parameter $x_{i}$

The error function of the refinement training of the surrogate model Testing error of EM centric multiphysics model 
$E_{T r}$

$E^{t}$

$\boldsymbol{F}_{E M}$

$\boldsymbol{F}_{M P}$

$f$

$\boldsymbol{f}_{A N N}$

$\boldsymbol{f}_{A N N 1}$

$\boldsymbol{f}_{A N N 2}$

$f_{c}$

$\boldsymbol{G}_{E M}$

$\boldsymbol{G}_{M P}$

$g_{i}{ }^{k}$

$\boldsymbol{g}^{k}$
Training error of EM centric multiphysics model

The the error function for $t^{t h}$ sub-model

The derivative information of the EM domain outputs w.r.t. the EM domain frequency parameter

The derivative information of the EM domain frequency parameter w.r.t. the multi-physics domain non-geometrical parameters

The frequency parameter

The neural network function

The neural network mapping function for first mapping module

The neural network mapping function for second mapping module

The frequency parameter of EM domain

The derivative information of the EM domain outputs w.r.t. the EM domain design parameters

The derivative information of the EM domain design parameters w.r.t. the multi-physics domain geometrical parameters

First order derivative of EM response w.r.t. the geometrical parameter $x_{i}$ at $k^{\text {th }}$ training sample

A vector containing all the values of $g_{i}{ }^{k}$ for $i=1,2, \cdots, N$ 


\begin{tabular}{|c|c|}
\hline$H_{i, i, q}^{k}$ & $\begin{array}{l}\text { The second-order derivative w.r.t. the geometrical parameter } x_{i} \text { at } \\
\text { the } k^{t h} \text { training sample and } q^{t h} \text { frequency point }\end{array}$ \\
\hline$H_{i, i}^{k}$ & $\begin{array}{l}\text { Second-order derivative of the EM response w.r. t. the geometrical } \\
\text { parameter } x_{i} \text { for } k^{\text {th }} \text { training sample }\end{array}$ \\
\hline $\boldsymbol{h}^{k}$ & $\begin{array}{l}\text { A vector containing all the unknown elements in the Hessian matrix } \\
\text { at } k^{t h} \text { training sample }\end{array}$ \\
\hline $\boldsymbol{I}_{k}$ & an index set containing the indices of the $N_{t}$ samples \\
\hline$i$ & Index number of the geometrical parameters \\
\hline$K$ & The stage index which is set to be 1 at the first training stage \\
\hline$k$ & $\begin{array}{l}\text { Index number of the training samples for modeling problem, the } \\
\text { index of optimization iterations for optimization problem }\end{array}$ \\
\hline$L_{i}$ & The number of divisions for the geometrical parameter $x_{i}$ \\
\hline $\boldsymbol{L}$ & $\begin{array}{l}\text { A vector representing the number of divisions (i.e., number of seg- } \\
\text { ments) for all the geometrical parameters }\end{array}$ \\
\hline $\boldsymbol{M}_{M P}$ & $\begin{array}{l}\text { The derivative information of the EM domain design parameters } \\
\text { w.r.t. the multi-physics domain non-geometrical parameters }\end{array}$ \\
\hline$m$ & Index number of the training samples \\
\hline$N$ & number of geometrical design parameters \\
\hline
\end{tabular}




\begin{tabular}{|c|c|}
\hline$N_{f}$ & The number of frequency points \\
\hline$N_{i}$ & The number of training samples that lie within the range of $\boldsymbol{X}^{t}$ \\
\hline$N_{s}$ & Number of training samples \\
\hline$N_{h}$ & The number of samples used to calculate the Hessian matrix \\
\hline$N_{t}$ & The number of samples in sub-range $\boldsymbol{X}^{t}$ \\
\hline$N_{o}$ & The order of the transfer function \\
\hline$N_{u}$ & The number of unknown elements in the Hessian matrix \\
\hline$n_{1}$ & The number of variables in $\boldsymbol{x}_{g}$ \\
\hline$n_{2}$ & The number of variables in $\boldsymbol{x}_{g m}$ \\
\hline$n_{3}$ & The number of variables in $\boldsymbol{x}_{m}$ \\
\hline$n$ & The total number of sub-models \\
\hline$n_{s}$ & The total number of the multiphysics training data \\
\hline$n_{w}$ & The number of unknowns of the weighting parameters \\
\hline$O_{k, m}$ & The distance between $\boldsymbol{x}^{k}$ and another training sample $\boldsymbol{x}^{m}$ \\
\hline$P_{i}$ & $\begin{array}{l}\text { The total value of the second-order derivative w.r.t. } x_{i} \text { at all train- } \\
\text { ing samples }\end{array}$ \\
\hline $\boldsymbol{P}_{S M}$ & The space mapping function \\
\hline
\end{tabular}


$p$

$p$

$p_{i}$

$\boldsymbol{p}_{c}$

$Q_{i}$

$\boldsymbol{Q}_{S M}$

$q$

$\boldsymbol{R}_{c}$

$R_{f}(\boldsymbol{x}, f)$

$R_{s}^{t}$

$R_{s}^{t, \text { new }}$

$R_{s}(\boldsymbol{x})$

$r_{i}$

$r$
Index number of $L$

The geometrical parameters of the fine model

The poles of the transfer function

The geometrical parameters of EM domain

The average value of the second-order derivative w.r.t. $x_{i}$ at all training samples

The implicit space mapping function

Other physics domain parameters which are considered as nongeometrical design variables

The response of the EM domain coarse model

Fine model response, such as EM simulation response, multi-physics

simulation response

The response of the $t^{\text {th }}$ sub-model after sub-model training

The revised response of the $t^{t h}$ sub-model after modification

The overall model response

The residues of the transfer function

$r^{\text {th }}$ value of $x_{j}$ in the reordered manner 
$r_{s} \quad$ An adjustment control index parameter

$S_{i}^{t, l} \quad$ The sigmoid function for modification at the upper boundary along the dimension of $x_{i}$ The sigmoid function for modification at the upper boundary along the dimension of $x_{i}$

$\boldsymbol{T} \quad$ A vector representing the average variation of the sensitivity of EM response w.r.t. all the geometrical parameters

$T_{i} \quad$ Average variation of sensitivity of EM response w.r.t. geometrical parameter $x_{i}$ at ranges of $D_{i}$

$T_{\min } \quad$ The minimum value of the vector $\boldsymbol{T}$

$T_{r} \quad$ The index set of training samples

$t_{l} \quad$ The index number of the neighboring sub-model bordering at the upper side of the $t^{\text {th }}$ sub-model along the direction of $x_{i}$

$t_{p} \quad$ The index number of the sub-models where $t_{p}=(t-1) L_{i}+p$, $p \in\left\{1,2, \cdots L_{i}\right\}$ 
$t_{u}$

$V_{j}^{r}$

$v$

$W$

$\boldsymbol{w}_{1}$

$\boldsymbol{w}_{2}$

$\boldsymbol{w}_{1}^{*}$

$\boldsymbol{w}_{2}^{*}$

$\boldsymbol{w}_{t}$

$\boldsymbol{w}_{i}$

$X_{i}^{\min }$

$X_{i}^{\max }$

$\boldsymbol{X}$

$\boldsymbol{X}_{s}^{k}$
The index number of the neighboring sub-model bordering at the upper side of the $t^{t h}$ sub-model along the direction of $x_{i}$

The corresponding $r^{t h}$ value of $x_{j}$ in the reordered manner

Index number of the training samples

The transition range of the sigmoid function

The internal weighting parameters of $\boldsymbol{f}_{A N N 1}$

The internal weighting parameters of $\boldsymbol{f}_{A N N 2}$

The internal weighting parameters of $\boldsymbol{f}_{A N N 1}$ after training process

The internal weighting parameters of $\boldsymbol{f}_{A N N 2}$ after training process

The ANN internal weighting parameters of the $t^{\text {th }}$ sub-model

The vector containing all the weighting parameters in the quadratic function $c_{i}$

Minimum value of the geometrical parameter $x_{i}$

Maximum value of the geometrical parameter $x_{i}$

A matrix of the wide geometrical range

The trained region of geometrical parameters of the surrogate model in the $k$ th iteration using the trust region algorithm 


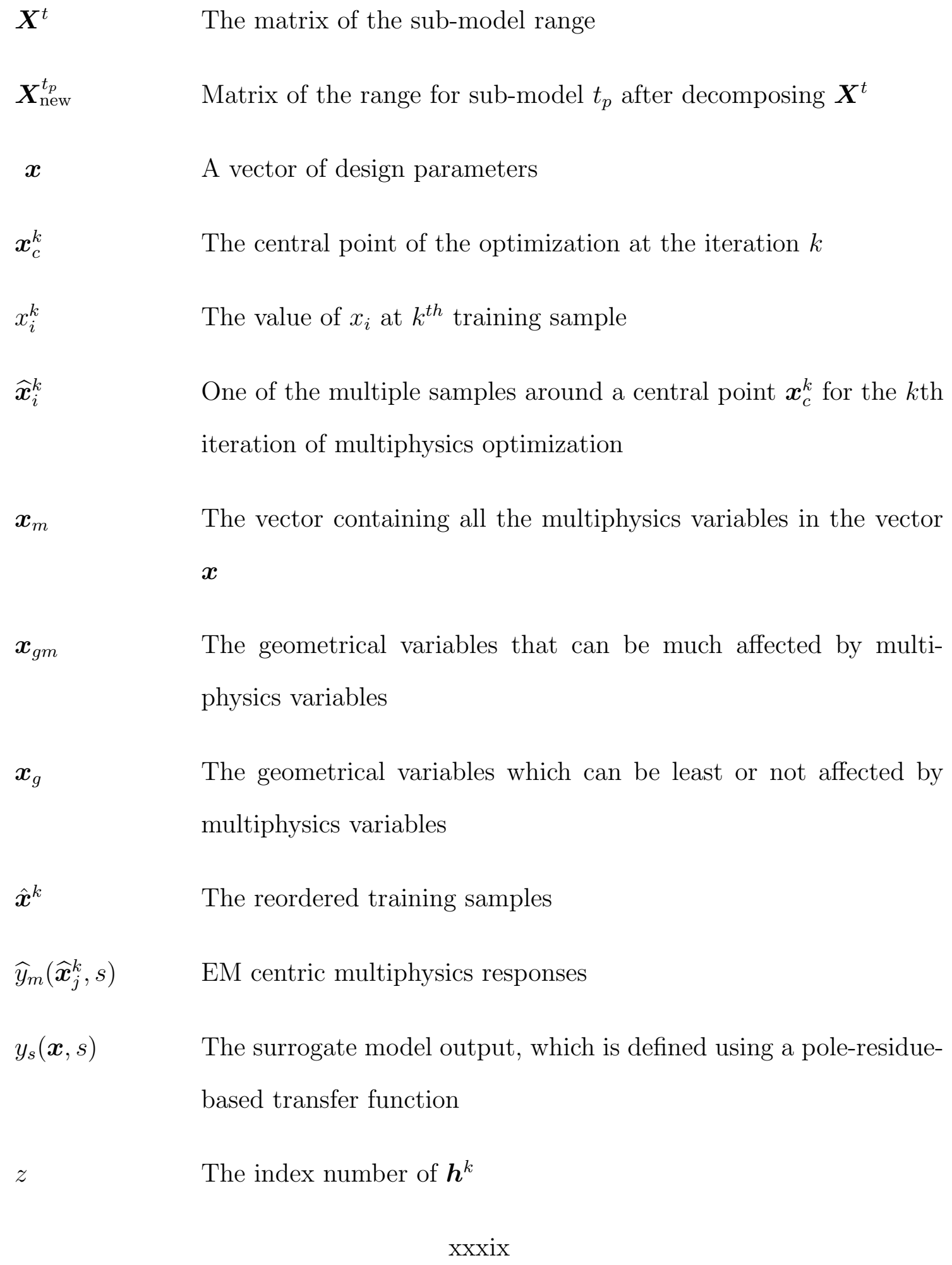

The index number of $\boldsymbol{h}^{k}$ 
$U\left(\widehat{y}_{m}\left(\boldsymbol{x}^{k}\right)\right)$

$U\left(y_{s}\left(\boldsymbol{x}^{k}\right)\right)$

$\eta_{e}$

$\eta_{c}$
User-defined threshold for testing error

A user-defined overlapping percentage

A index vector of the $t^{t h}$ sub-model

The index of divisions at the $i^{\text {th }}$ geometrical parameter for the submodel $t$ i.e., $\lambda_{i}^{t} \in\left\{1,2, \cdots, L_{i}\right\}$

The modification function along the direction of $x_{i}, i=1,2, \cdots, N$

The total modification function for the $t^{\text {th }}$ sub-model

The index set of the frequency samples

The trust radius in the $k$ th iteration

The range of each design variable in the $k$ th iteration

The objective function responses calculated using EM centric mul-

tiphysics simulation at $\boldsymbol{x}^{k}$

The objective function responses calculated using the surrogate

model at $\boldsymbol{x}^{k}$

The coefficients for the trust radius update when $r_{s}>0.85$

The coefficients for the trust radius update when $r_{s}<0.4$ 


\section{Chapter 1}

\section{Introduction}

\subsection{Background and Motivation}

For today's high performance radio frequency $(\mathrm{RF}) /$ microwave component and system design, we face the challenges of increasing complexity, tighter component tolerances, and shorter design cycles. To achieve more comprehensive and accurate microwave solutions, instead of equivalent circuit based simulation, it is desired to employ electromagnetic (EM) based simulation or even multiphysics based simulation. However, such simulations need to solve more complex equations and in general are more computationally expensive. While for EM/multiphysics simulation driven design, the computational cost of directly using EM/multiphysics simulator is even more expensive because EM/multiphysics design requires repetitive $\mathrm{EM} /$ multiphysics evaluations due to the adjustments of the values of design parameters. The demand for faster, more accurate and efficient computer-aided design $(\mathrm{CAD})$ techniques in the $\mathrm{RF} /$ microwave area becomes more and more ur-

gent. Parametric modeling [1] - [4] and surrogate based optimization techniques [5] 
- [8] can be used to efficiently and accurately perform the design of microwave components and devices. Parametric models can be developed using the information of EM/multiphysics responses as functions of design parameters. The developed parametric models allow faster simulations and optimizations with varying values of design parameters and subsequently can be implemented in high-level circuit and system designs [9] - [13].

In recent years, artificial neural networks (ANNs) have been recognized as a powerful technique for parametric modeling and design optimization of microwave components [14]-[18]. ANNs can be trained efficiently to learn the EM behaviors of microwave devices. The trained model provides an accurate and fast prediction of the EM behavior of microwave components with geometrical parameters as variables. ANNs can be used over and over again to increase microwave device design efficiency by avoiding repetitive and time-consuming EM simulations [16], [17].

As a further approach, the knowledge-based neural network has been introduced to improve the accuracy and reliability of modeling and design optimization [19] [22]. In the knowledge-based approach, models are developed using neural networks combined with prior knowledge such as analytical expressions, empirical models or equivalent circuits. These knowledge can help enhance the capability for learning and generalization of the overall parametric models [19], [20]. As a further development of ANN parametric modeling, sensitivity analysis has been used to speed up the model development process by utilizing EM sensitivity information to reduce the number of training data required for developing the model [23].

An advanced modeling approach, which combines neural networks and trans- 
fer functions (Neuro-TF), has been used to perform parametric modeling of EM responses [85], [116]. This approach can be used even if equivalent circuits or empirical models are unavailable. In [86], [126], a novel parametric modeling technique combining neural networks and pole-residue-based transfer functions has been used to develop parametric models of EM behaviors for microwave components. This method addresses the discontinuity issue of the pole-residue-based transfer function.

Space mapping (SM) technique [28] - [31] has gained recognition in CAD area. SM considers the existence of coarse models and fine models. The coarse models usually represent the equivalent circuits or the empirical functions. They are computationally very efficient while not accurate enough. The fine models, such as 3D EM simulator, are typically very accurate but computationally very intensive. By using SM techniques, we can combine the accuracy of the fine models with the computational efficiency of the coarse models. SM aims to use the fewest possible fine model evaluations by exploiting coarse models during the optimization process, thus accelerating the overall optimization. Efforts on space mapping have focused on several areas, such as implicit SM [28], aggressive SM [29], tuning SM [30], parallel SM [31] and SM with adjoint sensitivities [7].

Recently, parallel computation has been applied to EM optimization to speed up the intensive computational process. A parallel SM approach presented in [31], uses parallel data generation and parallel training to speed up the optimization. In [32], [33], alternative parallel approaches to gradient-based EM optimization are discussed. 
Multiphysics analysis has become a hot topic in recent years [34] - [42]. For high performance $\mathrm{RF} /$ microwave component and system design, besides the EM domain (single physics), we often require considerations of the operation in a real world multiphysics environment [34], [35] which includes other physics domains. Understanding the interaction between multiple physics domains is essential for an accurate system analysis. The EM centric multiphysics problem involves EM analysis coupled with the effects of other physics domains such as thermal and structural mechanics. EM centric multiphysics simulation of microwave components involves the simultaneous solutions of EM and other physics domains which can provide the accurate evaluation of EM behavior. The EM centric multiphysics analysis becomes necessary for a growing number of microwave components because EM single physics (EM only) analysis may not be sufficiently accurate in a real world [35], [41]. The computational cost of the multiphysics simulations is very expensive because it involves multiple domains, coupling between domains and often deals with the deformed structure. Design optimization of EM centric multiphysics behaviors of the microwave components is even more time-consuming since it requires repetitively multiphysics evaluations due to the adjustments of the values of design variables.

In this thesis, we propose new techniques to speed up the parametric modeling and design optimization of EM and multiphysics behaviors. A novel technique is proposed to develop a low-cost EM centric multiphysics parametric model for microwave components. To accelerate the multiphysics optimization process, we develop a novel parallel EM centric multiphysics optimization technique. As a further advancement, a novel decomposition technique to address the challenges of 
EM parametric modeling with geometrical changes in a large range.

\subsection{List of Contributions}

The main objective of this thesis is to speed up the parametric modeling and design optimization of EM and multiphysics behaviors of microwave components. Towards this objective, we have made several technical contributions in this thesis, listed as:

- A novel technique is proposed to develop a low-cost EM centric multiphysics parametric model for microwave components [34]. In the proposed method, we use SM techniques to combine the computational efficiency of EM single physics (EM only) simulation with the accuracy of the multiphysics simulation. The EM responses with respect to different values of geometrical parameters in non-deformed structures without considering other physics domains are regarded as coarse model. The coarse model is developed using the parametric modeling methods such as artificial neural networks (ANNs) or neuro-transfer function (Neuro-TF) techniques. The EM responses with geometrical and nongeometrical design parameters as variables in the practical deformed structures due to thermal and structural mechanical stress factors are regarded as fine model. The fine model represents the behavior of EM centric multiphysics responses. The proposed model includes the EM domain coarse model and two mapping neural networks to map the EM domain (single physics) to the multiphysics domain. Our proposed technique can achieve good accuracy for multiphysics parametric modeling with fewer multiphysics training data and less computational cost. 
- For the first time, a novel parallel EM centric multiphysics optimization technique is developed. In our proposed technique, the pole-residue-based transfer function is exploited to build an effective and robust surrogate model [42]. A group of modified quadratic mapping functions is formulated to map the relationships between pole/residues of the transfer function and the design variables. Multiple EM centric multiphysics evaluations are performed in parallel to generate the training samples for establishing the surrogate model. Using our proposed technique, the surrogate model can be valid in a relatively large neighborhood which makes an effective and large optimization update in each optimization iteration. The trust region algorithm is performed to guarantee the convergence of the proposed multiphysics optimization algorithm. Our proposed multiphysics optimization technique takes a small number of iterations to obtain the optimal EM centric multiphysics response.

- A novel decomposition technique to address the challenges of electromagnetic (EM) parametric modeling where the values of geometrical parameters change in a large range [60]. In this method, a systematic and automated algorithm based on second-order derivative information is proposed to decompose the overall geometrical range into a set of sub-ranges. Using the proposed technique, a smooth region is decomposed into a few large sub-regions while a highly nonlinear region is decomposed into many small sub-regions. The proposed technique provides an efficient mathematical methodology to perform the decomposition systematically and automatically. An artificial neural network (ANN) model with simple structure, hereby referred to as a sub-model, 
is developed with geometrical parameters as variables in each sub-region. When the values of geometrical parameters change from the region of one submodel to another sub-model, the discontinuity of EM responses is observed at the boundary between the adjacent sub-models. There are many sub-model boundaries in the overall model resulting in the complex multi-dimensional discontinuity problem. Thereby the overall model provided by directly combining all the trained sub-models cannot be used for design optimization. A sub-model modification process is proposed to solve this multi-dimensional discontinuity problem to obtain a continuous model over the entire region. Parallel data generation, parallel sub-model training, and parallel sub-model modification are proposed to speed up the model development process. Compared with standard modeling methods using a single model to cover the entire wide geometrical range, the proposed method can obtain better modelaccuracy with short model-development time. Three microwave examples are used to illustrate the validity of the proposed technique.

\subsection{Thesis Organization}

The rest of the thesis is organized as follows:

Chapter 2 presents a literature review of popularly used parametric modeling and optimization techniques for EM and multiphysics behaviors of microwave com-

ponents. An overview of ANN structure, ANN training, and knowledge based neural network techniques are presented. The pole-residue-based transfer function is reviewed and recent Neuro-TF parametric modeling method are also discussed. An 
overview of classical space mapping methods such as implicit space mapping, output space mapping, tuning space mapping and the recently developed coarse and fine mesh space mapping is reviewed. EM centric multiphysics simulation of microwave components with multiple physics domain analysis is presented. The decomposition techniques for wide range problem are reviewed.

Chapter 3 presents a novel technique to develop a low-cost EM centric multiphysics parametric model for microwave components. The proposed model includes the EM domain coarse model and two mapping neural networks to map the EM domain (single physics) to the multiphysics domain. Our proposed technique can achieve good accuracy for multiphysics parametric modeling with fewer multiphysics training data and less computational cost. This proposed technique is demonstrated by two microwave examples.

Chapter 4 presents a novel parallel EM centric multiphysics optimization technique. The pole-residue-based transfer function is exploited to build an effective and robust surrogate model. Our proposed multiphysics optimization technique takes a small number of iterations to obtain the optimal EM centric multiphysics response. Two microwave filter examples are used to demonstrate the validity of the proposed technique.

Chapter 5 presents a novel decomposition technique to address the challenges of EM parametric modeling with geometrical changes in a large range. The proposed method can obtain better model-accuracy with short model-development time. Three microwave examples are used to illustrate the validity of the proposed technique. 
Finally, Chapter 6 presents the conclusions and discussions on possible directions for future work. 


\section{Chapter 2}

\section{Literature Review}

For electromagnetic (EM) and multiphysics design, the computational cost of directly using EM/multiphysics simulator is very expensive because EM/multiphysics simulation-driven design requires repetitive EM/multiphysics evaluations due to the adjustments of the values of design parameters. Parametric modeling and optimization of EM and multiphysics behaviors are very essential parts of the design process of microwave components. A variety of parametric modeling and optimization techniques have been introduced for modeling EM/multiphysics behaviors of passive and active components. This chapter reviews frequently used parametric modeling and optimization techniques.

\subsection{Artificial Neural Network}

In recent years, artificial neural networks (ANNs) have emerged as powerful techniques for parametric modeling and design optimization of EM based microwave components with geometrical parameters as variables [14] - [6]. For EM-simulationdriven design, the computational cost of directly using fine models can be very 
expensive because EM-simulation-driven design requires repetitive EM evaluations due to the adjustments of the values of the geometrical parameters. Neural networks are trained to learn the relationships between EM responses and geometrical parameters. The trained model provides accurate and fast prediction of the EM behavior of microwave components with geometrical parameters as variables, and can be subsequently implemented in high-level circuit and system design [16] - [18].

In the past decades, significant advances have been made in development of accurate and efficient ANNs to overcome RF/microwave modeling and design difficulties [6]. Researchers have investigated a variety of important techniques utilizing the ability of ANNs to perform parametric modeling and optimization of microwave components and circuits, such as microwave FETs [43], [44], HBTs [45], [46], HEMTs [47], amplifiers [48], [49], high-speed VLSI interconnects [50], [51], multilayer circuit packages [52], embedded passives [20], [53], antennas [54], coplanar waveguide (CPW) components [55], [56], filters [57]-[59], mixers [49], laser diodes [61], waveguides [62], tunable cavity filters [34], high power filters [35].

A variety of ANN structures have been developed during past several years. Pure ANNs such as multilayer perceptron (MLP) neural networks [63], dynamic neural networks [64], [65], time-delay neural networks [66], recurrent neural networks [49], and the recently introduced deep neural networks [67] have been used for directly modeling the linear/nonlinear relationships between the model inputs and outputs. 


\subsubsection{Neural Network Structures}

Multilayer Perceptrons (MLP) are the most popular type of neural networks, which are used in many different microwave applications [43], [63]. In the structure of MLP, the neurons are grouped into layers. The first layer is called input layer and

the last layer is called output layer, because they represent the inputs and outputs of the overall neural network function. The remaining layers in between are called hidden layers. As described, the MLP neural networks consists of one input layer, one or more hidden layers, and one output layer, demonstrated in Fig. 2.1.

The MLP neural networks are the feed-forward neural networks where the external inputs are fed to the first layer input neurons, the outputs of the first layer input neurons are fed to the second layer hidden neurons, and so on, and until the output neurons obtain the final solutions which are the model outputs.

Based on the universal approximation theorem for MLP neuron networks was proved in 1989 by Cybenko and Hornik et al [68], [69] , a three layer perceptron can approximate any arbitrary nonlinear, continuous, multi-dimensional function for any desired accuracy. When designing an ANN structure, there are typically two important factors needed to be considered, one is the number of hidden layers and the other one is number of hidden neurons in each hidden layer. The ongoing research for determining the number of hidden neurons includes algorithms such as constructive algorithms [70], network pruning [71], regularization [72], and automatic model generation [73], to match the neural network model non-linearity with problem non-linearity. For obtaining the optimal number of hidden layers, structural optimization algorithms based on the training data have been investigated. 


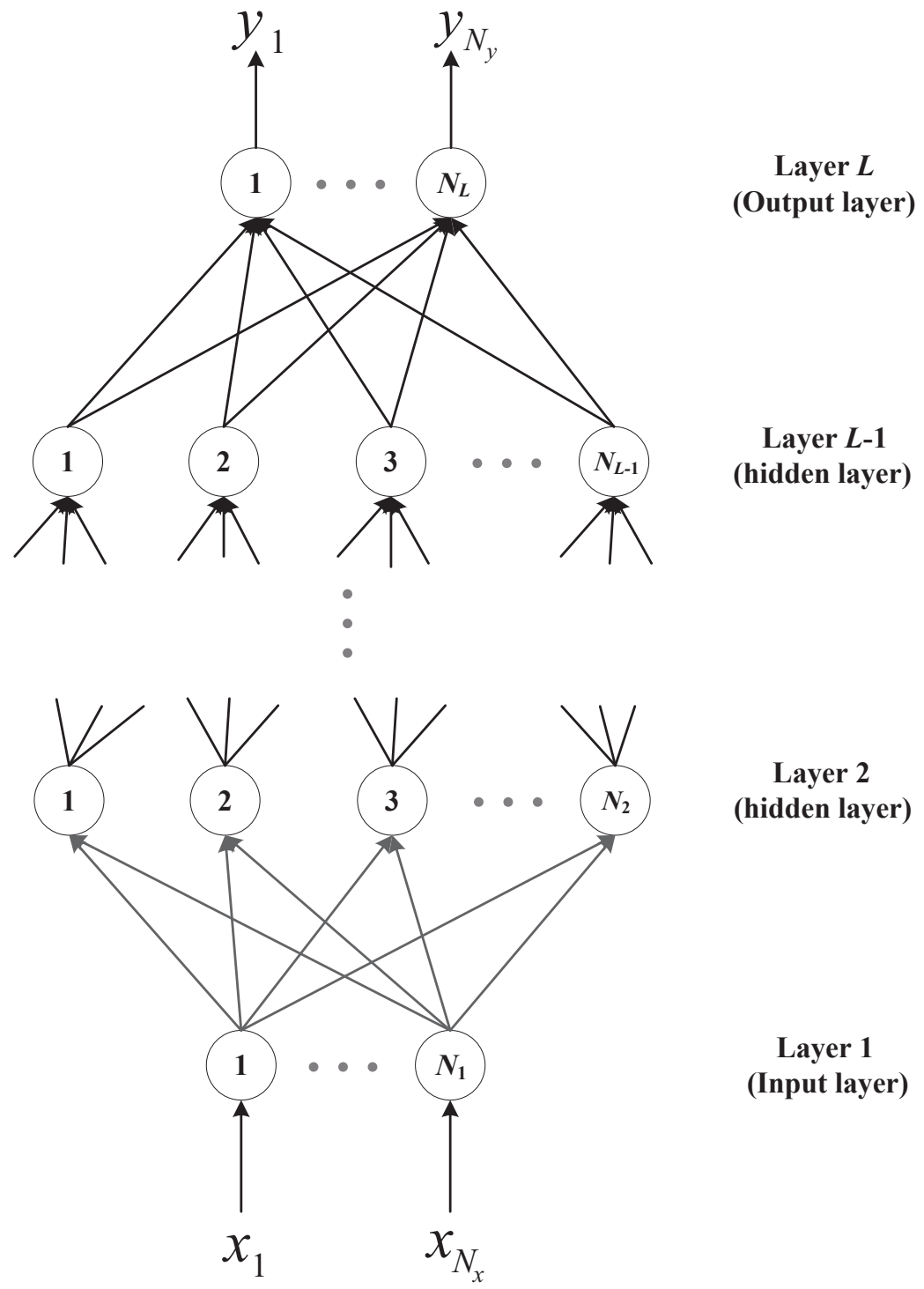

Figure 2.1: The multilayer perceptron (MLP) structure cosisting of one input layer, one or more hidden layers, and one output layer.

Typically, for microwave passive and active device parametric modeling problem, a three or four layer perceptrons are most commonly used. Three-layer perceptrons 
are usually preferred when generalization capability of the function approximation is a major concern [74], because fewer hidden neurons are needed in the resulting neural network. On the other hand, considering four layer perceptrons have better mapping capability, they are preferred for pattern classification tasks where decision boundaries need to be defined [75].

Radial basis function (RBF) neural network is another popular neural network structure which has a single hidden layer using radial basis activation functions for hidden neurons [76], [77]. RBF neurons networks have been applied to many $\mathrm{RF} /$ microwave modeling applications such as modeling of inter-modulation distortion behaviors of transistors.

Wavelet neural networks inspired by combining wavelet theory with neural networks use wavelet functions as hidden neuron activation functions. The wavelet network has been used in modeling passive and active microwave components for microwave circuit design $[78]-[80]$.

Recurrent neural network (RNN) is a new type of neural network structure that allows time-domain behaviors of a dynamic system is described. This type of neural network is established depending not only on the present inputs but also on the history of the system states and inputs [81]-[83]. A recurrent neural network structure with feedback of delayed neural network is shown in Fig. 2.2. The RNN structure suits the time domain modeling problems such as FDTD solutions in EM modeling and dynamic system control. 


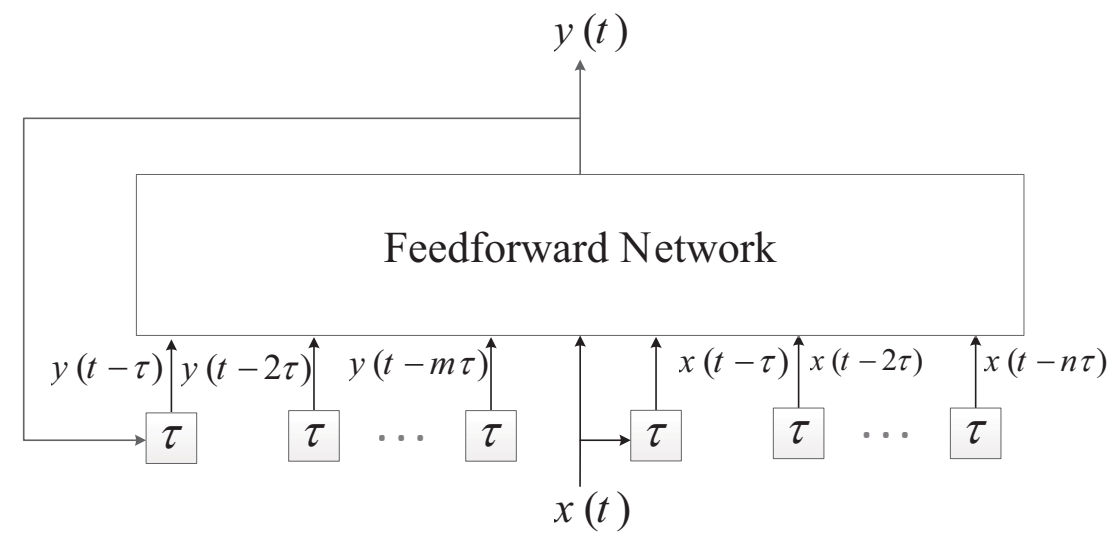

Figure 2.2: A recurrent neural network structure with feedback of delayed neural network output.

\subsubsection{Neural Network Training}

A neural network in the beginning stage cannot represent any $\mathrm{RF} /$ microwave behavior unless it is well trained with $\mathrm{RF} /$ microwave data. To develop an accurate neural network model, the typical model development process consists of problem statement, data generation (including training, validation, and testing data), data scaling, training, validation, and testing.

Let $\boldsymbol{x}$ represent a vector containing the design parameters of a $\mathrm{RF} /$ microwave component. Let $\boldsymbol{y}$ represent a vector containing the EM/multiphysics behavior of the component. The relationship between $\boldsymbol{x}$ and $\boldsymbol{y}$ can be represent by a neural network function, formulated as

$$
\boldsymbol{y}=\boldsymbol{f}_{A N N}(\boldsymbol{x}, \boldsymbol{w})
$$

where $\boldsymbol{w}$ represents a vector containing all neural network weighting parameters. 
Once we define the input and output of the neural networks, the next step toward neural network development is data generation. The data can be generated either from measurements or simulations based on the modeling problem. There are several data sampling methods such as uniform grid distribution, non-grid distribution, design of experiments (DOE), star distribution and random distribution. The DOE sampling method provides a reasonable distribution of simulation points by spreading the information throughout the region of the input variables.

After data generation, a fast and accurate neural model is developed by training the neural network function $\boldsymbol{f}_{A N N}$ with the training samples. The training data is denoted by input-output sample pairs $\left\{\left(\boldsymbol{x}_{k}, \boldsymbol{d}_{k}\right), k \in T_{r}\right\}$, where $\boldsymbol{d}_{k}$ represents the measured/simulated output data for the input $\boldsymbol{x}_{k}$, and $T_{r}$ represents the index set of training data.

For training purpose, the error function $E(\boldsymbol{w})$ is formulated as,

$$
\left.E(\boldsymbol{w})=\frac{1}{2} \sum_{k \in T_{r}} \| \boldsymbol{f}_{A N N}\left(\boldsymbol{x}_{k}, \boldsymbol{w}\right)-\boldsymbol{d}_{k}\right) \|^{2}
$$

The primary objective of neural network training is to minimize $E(\boldsymbol{w})$ by adjusting the weighting parameters $\boldsymbol{w}$.

A variety of training algorithms have been developed during past years. The commonly used neural network training algorithms in RF/microwave applications include gradient-based training algorithm such as back propagation (BP), conjugate gradient, Quasi-Newton, Levenberg-Marquardt. Global training methods such as genetic algorithms and simulated annealing can be used for minimizing the training and testing error of the neural network model but at the cost of increasing the 
training time. In general, we can train the neural network by two categories training process, one is sample-by-sample training which is also called online training where the weighting parameters are updated each time a training sample is fed to the neural network; the other one is batch-model training process which is also known as offline training where the weighting parameters are updated after all the training samples are used. In microwave passive and active device modeling and optimization case, the batch-mode is preferred for its higher efficiency.

After training, an independent set of data which is never used in training process is used to test the accuracy of the neural network model. In general, the test data should be generated within the same input range as the training data. If both training and testing results are satisfied with the user desired accuracy, the neural network model training process is finished and the well trained model can be used for higher level design. The trained ANN model can provide fast and accurate model evaluation replacing the original detailed slow EM/multiphysics simulators. The benefit of the neural network model is more significant when the model is used in design process such as design optimization, Monte Carlo analysis and yield optimization [84]. The more we use the model, the more benefits we have.

\subsection{Knowledge-Based Neural Network}

As a further approach, the knowledge-based neural network (KBNN) is also studied where microwave empirical or semi-analytical information is incorporated into the model structure. The microwave knowledge complements the capability of learning and generalization of neural networks by providing additional information such as 


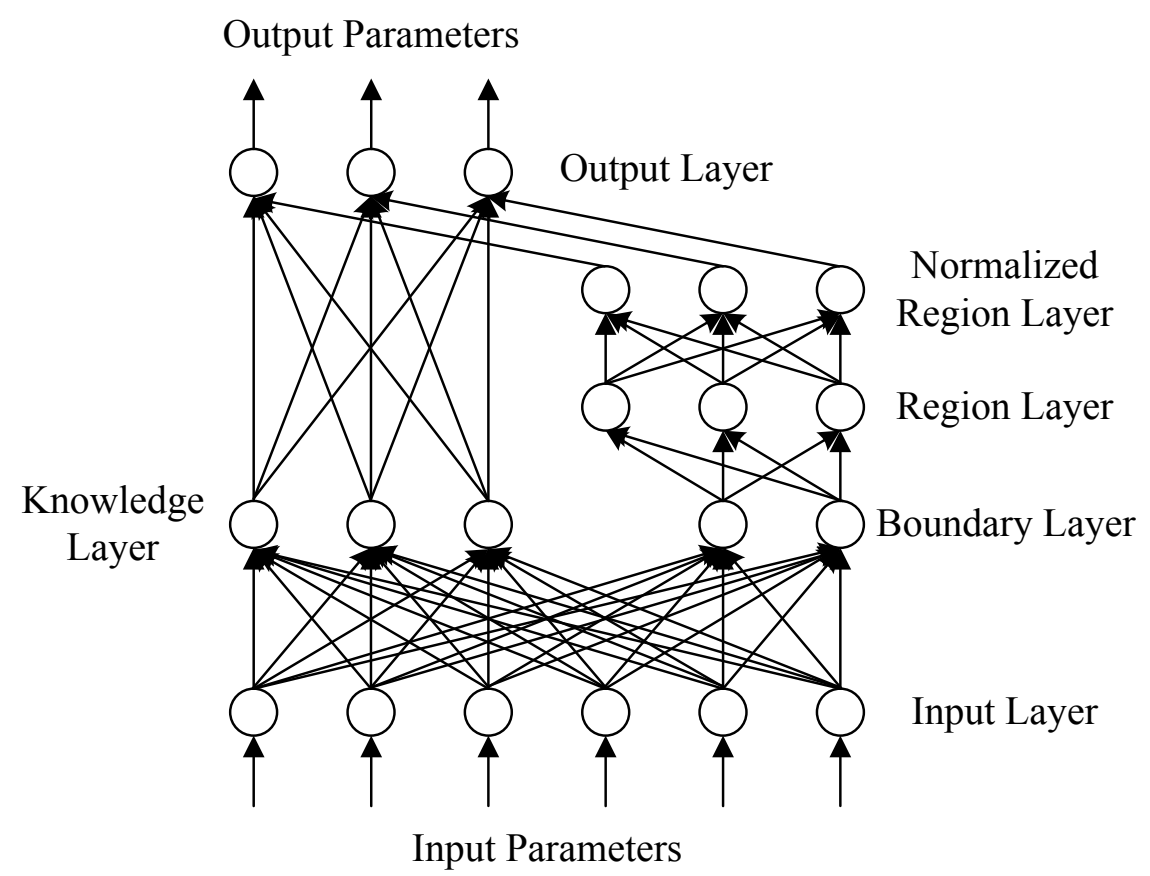

Figure 2.3: Illustration of the structure of knowledge-based neural network (KBNN) $[43]$.

analytical expressions [19], empirical models [20], or equivalent circuits [21], [22]. The idea of the knowledge-based model is to exploit existing knowledge in the form of empirical or equivalent circuit models together with neural networks to develop a faster and more accurate model. The structure of knowledge-based neural network is illustrated in Fig. 2.3.

In the KBNN structure, the prior $\mathrm{RF} /$ microwave information is embedded into the overall neural network internal section which consists of six layers, i.e., the input layer, the knowledge layer, the boundary layer, the region layer, the normalized region layer, and the output layer. The knowledge layer contains the microwave knowledge which provides the additional information to the original modeling prob- 
lem, which may not be adequately represented by limited training data. The boundary layer can incorporate knowledge in the form of problem dependent boundary functions. The region layer is used to construct regions from boundary neurons. The normalized region layer contains rational function based neurons to normalize the outputs of the region layer [43].

\subsection{Combined Neural Networks and Transfer Func- tion}

Recently, advanced modeling approach, which combines neural networks and transfer functions (neuro-TF), was developed to perform parametric modeling of EM responses [20], [85], [15]. This approach can be used even if accurate equivalent circuits or empirical models are unavailable. In this method, transfer functions are used to represent the EM responses of passive components versus frequency.

The model consists of transfer functions and neural networks. The outputs of the overall model are the $S$-parameters of the EM behavior of microwave components and the inputs of the model are geometrical variables of the EM structure and frequency. As the values of geometrical parameters change, the coefficients of transfer functions change accordingly. Due to the relationship between the coefficients of transfer functions and the geometrical parameters is nonlinear and unknown, neural networks are used to learn and represent this nonlinear relationship. The initial training data of neural networks are obtained by the vector fitting technique [87]. With vector fitting, the coefficients of transfer functions corresponding to a given set of EM responses are obtained. The neural networks are trained to learn the 


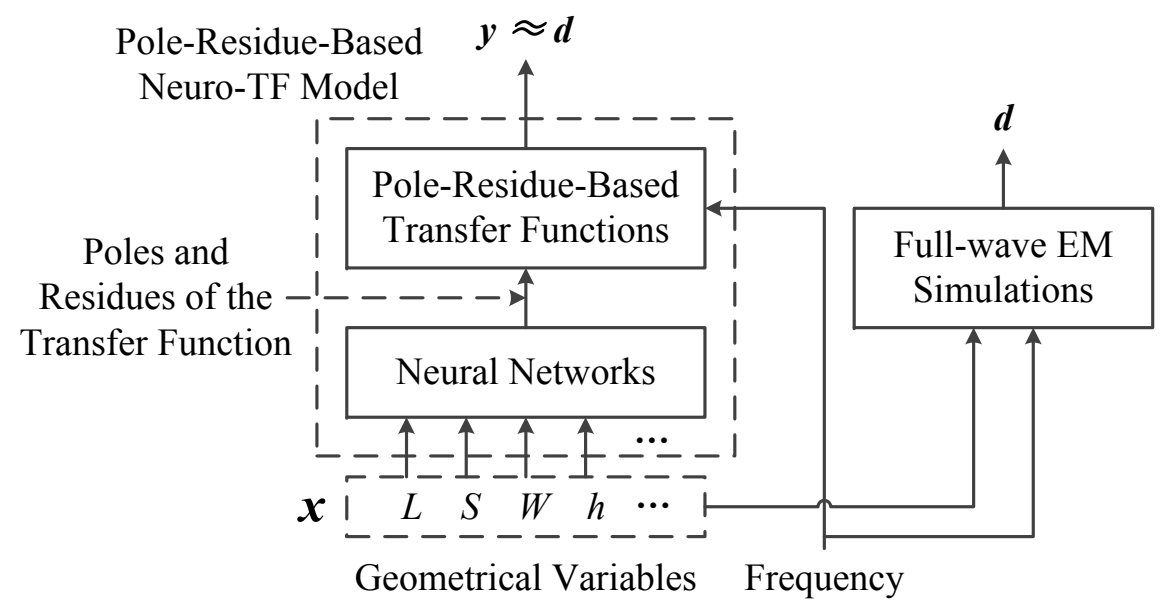

Figure 2.4: The structure of the pole-residue-based neuro-TF model. $\boldsymbol{x}$ represents the geometrical variables. $\boldsymbol{y}$ represents real and imaginary parts of the outputs of the pole-residue-based transfer function (e.g., S-parameters). $\boldsymbol{d}$ represents the outputs of the EM simulations.

nonlinear mapping between geometrical parameters and the coefficients of transfer functions.

In [86], a combined neural network and pole-residue-based transfer function models for parametric modeling of electromagnetic (EM) behavior of microwave components is presented. The structure of the pole-residue-based neuro-TF model is illustrated in Fig. 2.4. The model consists of pole-residue-based transfer functions and neural networks. The outputs of the overall model are the $S$-parameters of the EM behavior of microwave components and the inputs of the model are geometrical variables of the EM structure and frequency. Let $\boldsymbol{x}$ be a vector containing the geometrical variables, representing the inputs of the overall model. Let the frequency response $H(s)$ be a function of pole/residues, which is defined using a 
pole-residue-based transfer function as follows,

$$
H(s)=\sum_{i=1}^{N} \frac{r_{i}}{s-p_{i}}
$$

where $p_{i}$ and $r_{i}$ represent the poles and residues of the transfer function respectively, and $N$ represents the order of the transfer function.

As the values of geometrical parameters change, the pole/residues change accordingly. Due to the relationship between the pole/residues and the geometrical parameters is nonlinear and unknown, neural networks are used to learn and represent the nonlinear relationships. The three layer MLP is used as the neural network structure with linear functions as the output layer activation functions and sigmoid functions as the hidden layer activation functions. The initial training data of neural networks are obtained by the vector fitting technique [87]. With vector fitting, the poles and residues of the transfer function corresponding to a given set of EM responses is obtained. The neural networks are trained to learn the nonlinear mapping between $\boldsymbol{x}$ and the pole/residues. Let $\boldsymbol{y}$ be a vector representing real and imaginary parts of the outputs of the pole-residue-based transfer function. Let $\boldsymbol{d}$ be a vector representing the outputs of the EM simulations (e.g., real and imaginary parts of $S$-parameters). The objective here is to minimize the error between $\boldsymbol{y}$ and $\boldsymbol{d}$ for different $\boldsymbol{x}$, by adjusting the neural network internal weights. This method can obtain better accuracy in challenging applications involving high dimension of geometrical parameter space and large geometrical variations.

For constructing the combined neural networks and transfer function model, one of the major issue is the discontinuity of pole/residues in transfer functions with 
respect to the geometrical variables. When geometrical variations are large, the corresponding EM responses will lead to different orders of transfer functions. In [86], a pole-residue tracking technique solving the problem due to the change in the order of transfer functions has been presented. The main purpose for this technique is to add groups of new pole/residues to bridge the gap of transfer function orders between different geometrical samples while keeping the responses of the transfer functions unchanged. In this way, the transfer functions of constant order with respect to all geometrical samples are finally obtained, the non-uniqueness problem of pole/residues is solved.

\subsection{Space Mapping}

Space mapping (SM) techniques [28]-[31] have gained recognition in microwave computer-aided design (CAD) area addressing the growing computational challenges in 3D field optimization with geometrical parameters as variables. Space mapping assumes the existence of fine and coarse models [24], [28]. The fine models are usually very accurate but CPU intensive such as 3D field electromagnetic (EM) simulations, while the coarse models are typically empirical functions or equivalent circuits, which are computationally very efficient but not very accurate. Space mapping technique allows expensive EM optimizations to be performed efficiently with the help of fast and approximate surrogates [30], [31]. Fig. 2.5 shows the illustration of the space mapping concept. Typically, space mapping algorithms provide excellent results after a few evaluations of the fine model.

Efforts on space mapping have focused on several areas, such as implicit space 


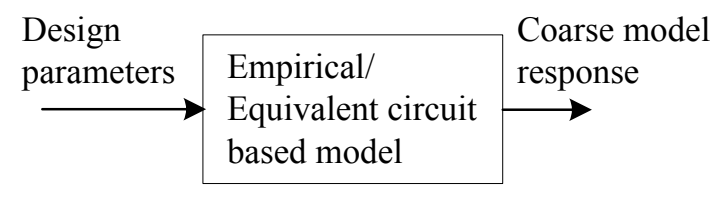

Coarse model

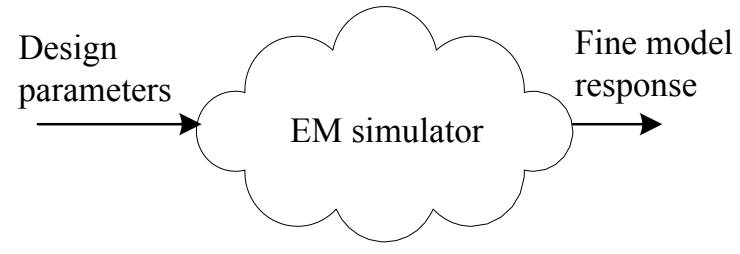

Fine model

\section{Parameter extraction}

(Extract mapping to match fine model and coarse model)
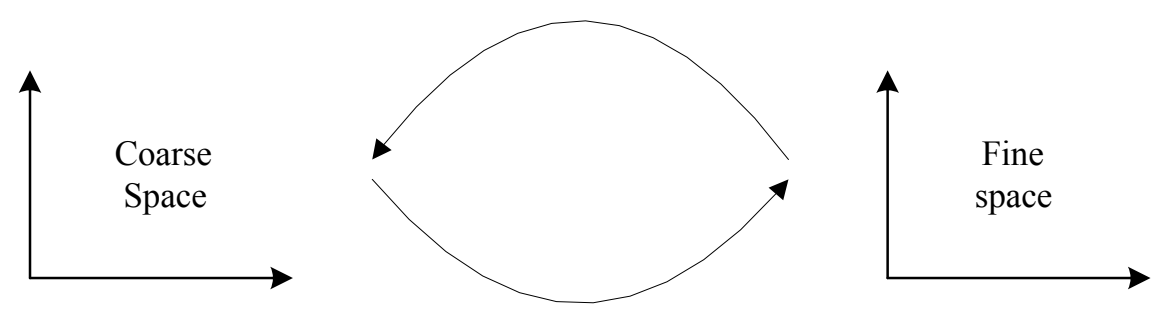

\section{Surrogate Optimization}

(Optimization update)

Figure 2.5: The illustration of space mapping concept.

mapping [88], [89], output space mapping [90],[8], neural space mapping [91]-[95], generalized space mapping [96], tuning space mapping [30], [97], portable space mapping [98], parallel space mapping [31], coarse and fine mesh space mapping [99], [100]. Recent improvements in space mapping such as constrained parameter extraction using implicit space mapping [28], space mapping optimization using EMbased adjoint sensitivity [7], and fast EM modeling using shape-preserving response prediction and space mapping [101] focus on reducing the number of fine model evaluations. 


\subsubsection{Space Mapping Concept}

Let $\boldsymbol{R}_{f}\left(\boldsymbol{x}_{f}\right)$ denotes the response vector of a fine model corresponding to a vector of design variables $\boldsymbol{x}_{f}$. The original optimization problem is formulated as follows

$$
\boldsymbol{x}_{f}^{*}=\arg \min _{\boldsymbol{x}_{f}} U\left(\boldsymbol{R}_{f}\left(\boldsymbol{x}_{f}\right)\right)
$$

where $U$ is a suitable objective function, which represents the error function of $\boldsymbol{R}_{f}\left(\boldsymbol{x}_{f}\right)$ with respect to the design specifications; $\boldsymbol{x}_{f}^{*}$ is the optimal fine model design to be found. We assume that solving problem (2.4) by means of direct EM optimization is computationally expensive. Instead, we exploit an inexpensive surrogate model; e.g., we establish a surrogate model which combines the coarse model with the input mapping function as

$$
\boldsymbol{x}_{c}=\boldsymbol{P}_{S M}\left(\boldsymbol{x}_{f}\right)
$$

such that

$$
\boldsymbol{R}_{s}\left(\boldsymbol{x}_{f}\right)=\boldsymbol{R}_{c}\left(\boldsymbol{x}_{c}\right)=\boldsymbol{R}_{c}\left(\boldsymbol{P}_{S M}\left(\boldsymbol{x}_{f}\right)\right)
$$

where $\boldsymbol{x}_{c}$ is a vector of design variables of the coarse model and $\boldsymbol{R}_{c}\left(\boldsymbol{x}_{c}\right)$ represents the

response vector of the coarse model corresponding to $\boldsymbol{x}_{c} . \boldsymbol{R}_{s}\left(\boldsymbol{x}_{f}\right)$ is a response vector of the surrogate model and $\boldsymbol{x}_{f}$ is a vector containing all the design optimization variables. $\boldsymbol{P}_{S M}$ represents the space mapping function. The surrogate is trained to be very close to the fine model as

$$
\boldsymbol{R}_{c}\left(\boldsymbol{P}_{S M}\left(\boldsymbol{x}_{f}\right)\right) \approx \boldsymbol{R}_{f}\left(\boldsymbol{x}_{f}\right)
$$



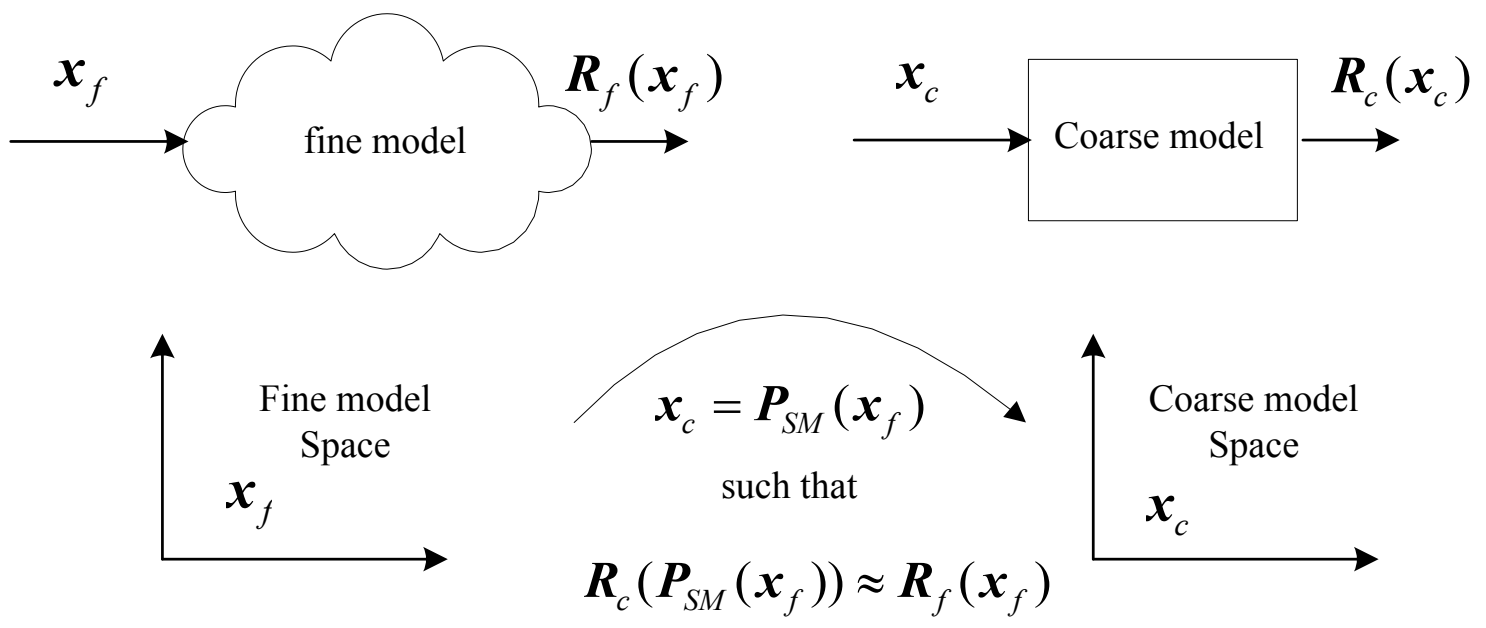

Figure 2.6: The mathematical representation of the space mapping methodology.

Thus, the design optimization using surrogate model can represent that using fine model described in (2.4). The optimal solution of the surrogate model is denoted as

$$
\boldsymbol{x}_{f}^{*}=\arg \min _{\boldsymbol{x}_{f}} U\left(\boldsymbol{R}_{c}\left(\boldsymbol{P}_{S M}\left(\boldsymbol{x}_{f}\right)\right)\right)
$$

Fig. 2.6 shows the mathematical representation of the space mapping methodology presented in the reference [102].

\subsubsection{Input Space Mapping}

At the beginning of the space mapping concept is presented, input space mapping is introduced as the most standard space mapping methodology [25], [102]. Input space mapping focuses on reducing the misalignment between the fine and coarse models by establishing a mapping between the input spaces (e.g., design parameter spaces) of the fine and coarse models. Input mapping with the linear mapping 
function is also called as original space mapping, defined as

$$
\boldsymbol{x}_{c}=\boldsymbol{P}_{S M}\left(\boldsymbol{x}_{f}\right)=\boldsymbol{B}_{S M} \boldsymbol{x}_{f}+\boldsymbol{c}_{S M}
$$

where $\boldsymbol{B}_{S M}$ and $\boldsymbol{c}_{S M}$ represent the coefficients of a linear mapping function.

\subsubsection{Implicit Space Mapping}

Implicit space mapping [88], [89] explores the flexibility of the preassigned parameter such as dielectric constant, substrate height in the design optimization process. Selected preassigned parameters (e.g., dielectric constant and substrate height) are extracted to match the coarse and fine models. The idea of using preassigned parameters was introduced in the reference [89] within an expanded space mapping design framework. This method selects certain key preassigned parameters based on sensitivity analysis of the coarse model. These parameters are extracted to match corresponding coarse and fine models. A mapping from optimization parameters to preassigned parameters is then established. Let $\boldsymbol{x}_{\text {aux }}$ represent the auxiliary parameters (i.e., preassigned parameters) and $\boldsymbol{R}_{c}\left(\boldsymbol{x}_{c}, \boldsymbol{x}_{\text {aux }}\right)$ represent the coarse model response. As illustrated in Fig. 2.7, implicit space mapping aims at establishing an implicit mapping $\boldsymbol{Q}_{S M}$ between the spaces $\boldsymbol{x}_{f}, \boldsymbol{x}_{c}$, and $\boldsymbol{x}_{a u x}$,

$$
\boldsymbol{Q}_{S M}\left(\boldsymbol{x}_{f}, \boldsymbol{x}_{c}, \boldsymbol{x}_{a u x}\right)=0
$$

such that

$$
\boldsymbol{R}_{f}\left(\boldsymbol{x}_{f}\right) \approx \boldsymbol{R}_{c}\left(\boldsymbol{x}_{c}, \boldsymbol{x}_{a u x}\right)
$$




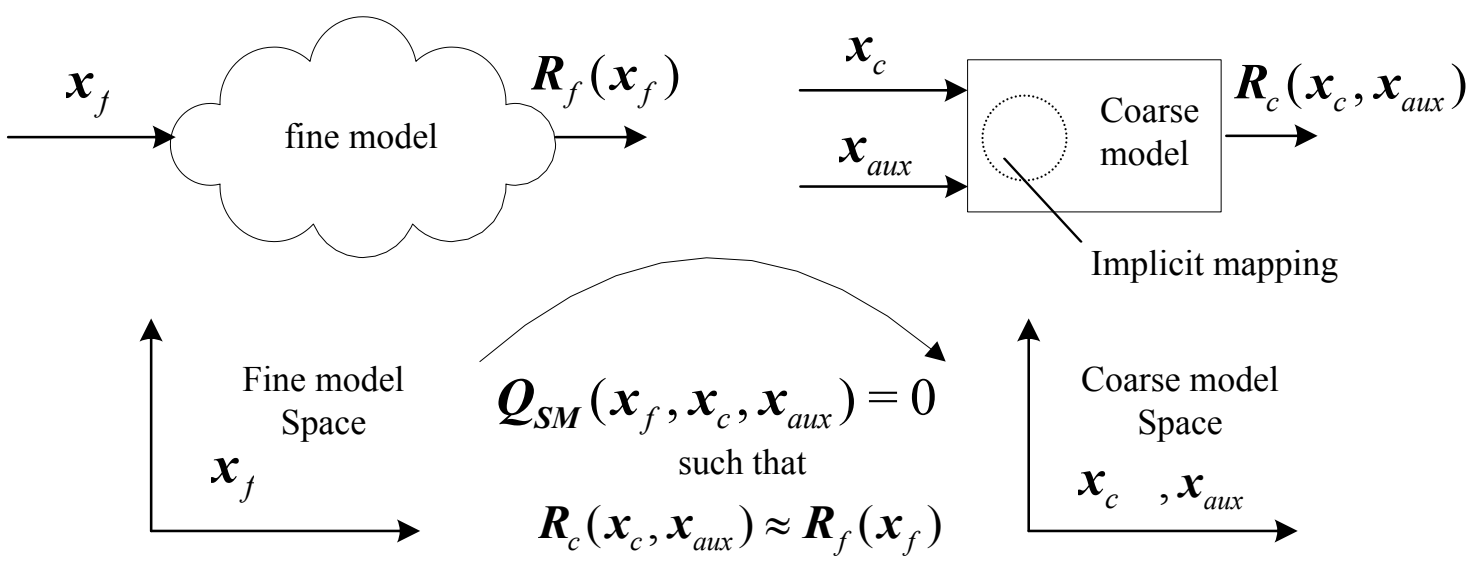

Figure 2.7: The mathematical representation of the implicit space mapping methodology.

Implicit mapping produces a good match between the coarse and fine models in the first iteration when input space mapping alone cannot obtain a good match.

\subsubsection{Output Space Mapping}

It is important for the performance of space mapping that the surrogate model response can represent the fine model response well with a proper input or implicit mapping. However, with only input or implicit space mapping, the surrogate model response may not be enough to precisely represent the fine model response. Therefore, a so-called output space mapping has been introduced [96], [103]. Output space mapping enhances the surrogate model by a correction term which is the difference between the fine model and the original space mapping response at the current iteration, [104]-[106], formulated as,

$$
\boldsymbol{R}_{s}\left(\boldsymbol{x}_{f}\right)=\boldsymbol{P}_{\text {corr }}\left(\boldsymbol{x}_{f}\right)+\boldsymbol{R}_{c}\left(\boldsymbol{P}_{S M}\left(\boldsymbol{x}_{f}\right)\right)
$$


where $\boldsymbol{P}_{\text {corr }}\left(\boldsymbol{x}_{f}\right)$ represents the added correction term at the output space in surrogate model.

The surrogate model is further enhanced by using the Jacobian of the surrogate model (to satisfy first order consistency between the surrogate model and fine model at the current design). If the misalignment between the fine and coarse models is not significant, SM-based optimization algorithms typically provide excellent results after only a few evaluations of the fine model. Having a large number of space mapping types results in a even larger number of combinations for space mapping. So the need to choose the suitable coarse model and SM approach for a design problem is crucial. A suitable choice of SM approach requires both knowledge of the problem and engineering experience.

\subsubsection{Tuning Space Mapping}

Tuning space mapping (TSM) [26], [97] is a special type of SM technique that caters to tuning of EM structures. The surrogate model is replaced by a tuning model which introduces circuit components in to the fine model structure. The tuning model is optimized within a circuit simulator. With the optimal tuning parameters, thus obtained, they are mapped or transformed into the design variables using fast space-mapping surrogate or analytical formulas if available. Tuning models require a significant engineering expertise for a successful implementation of the optimization process using TSM approach. 


\subsubsection{Neuro Space Mapping}

The most frequently used space mapping technique is developed to use linear mappings to establish a mathematical link between the coarse model and the fine data. However, when the modeling range becomes large, linear mappings only are not enough. Neuro space mapping is presented to solve this problem [18]. The neural networks are used to provide a nonlinear computational approach to bridge the gap between the empirical/equivalent circuit model and the new EM simulation data. This is achieved with the space mapping concept using neural networks to represent the nonlinear mappings between the empirical/equivalent circuit model and the EM data. Fig. 2.8 illustrate the neuro space mapping concept. Extrapolation capability is also enhanced because of the embedded knowledge in the model [43]. Several approaches for the structure selection of the neuro space mapping model are described in the existing literature [91]-[95].

\subsubsection{Parallel Space Mapping}

Parallel computation is a powerful method to speed up intensive computational processes and utilize computer's number crunching ability more effectively [107]. Many researches on parallel method have been done in several areas [108]-[115]. Parallel automatic model generation technique is proposed in the reference [108], using parallel adaptive sampling and parallel data generation to save the model development

time. In the reference [113], the EM data is generated by running multiple EM simulations in parallel on a multi-processor environment. This technique is used to speed up the design optimization of microwave circuits. In the references [114], 


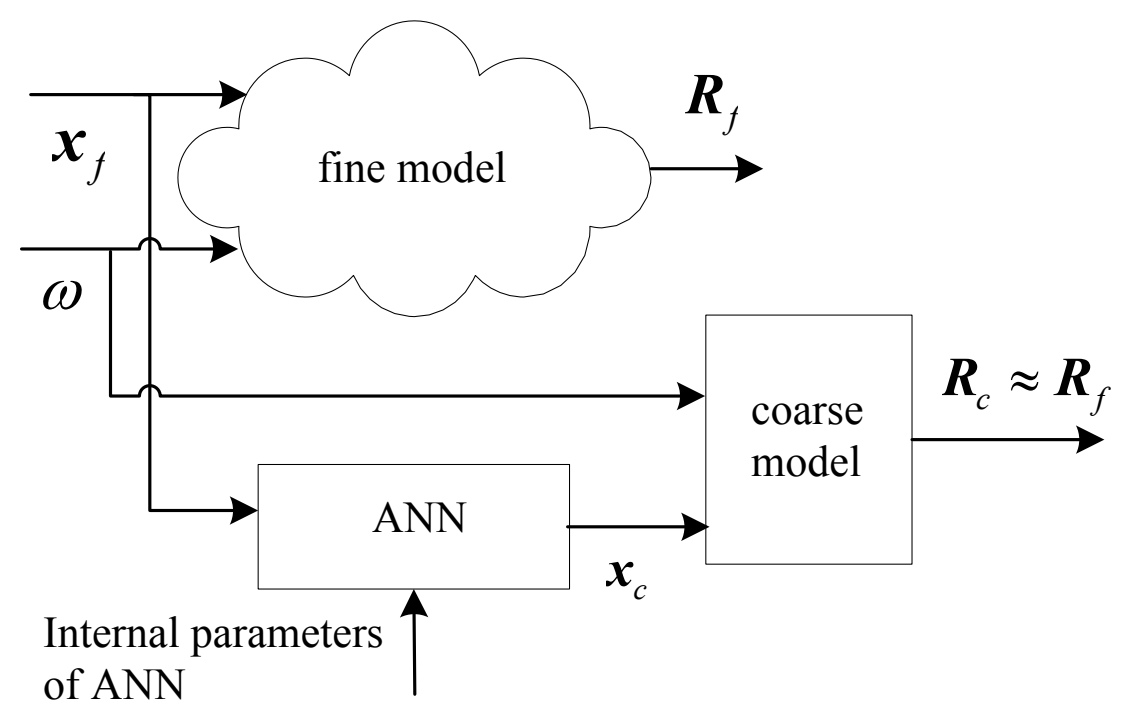

Figure 2.8: The illustration of neuro space mapping concept

[115], distributed fine model evaluation technique has been presented.

In the reference [31], a parallel space mapping optimization algorithm is presented. The surrogate model developed in each iteration is trained to match the fine model at multiple points, thereby making the surrogate model to be valid in a larger neighborhood. The formulation of multi-point surrogate model training is inherently suited to and implemented through parallel computation. This includes multiple fine model evaluation in parallel and multi-point surrogate training using parallel algorithm.

\subsection{EM Centric Multiphysics Simulations}

For high performance RF/microwave component and system design, besides the EM domain (single physics), we often require considerations of the operation in a 
real world multiphysics environment [116] - [120] which includes other physics domains. Understanding the interaction between multiple physics domains is essential for an accurate system analysis. We consider the EM centric multiphysics problem which involves EM analysis coupled with the effects of other physics domains such as thermal and structural mechanics. EM centric multiphysics simulation of microwave components involves the simultaneous solutions of EM and other physics domains which can provide the accurate evaluation of EM behavior. The EM centric multiphysics analysis becomes necessary for a growing number of microwave components and systems because EM single physics (EM only) analysis may not be sufficiently accurate in a real world. As examples of multiphysics related research, recently a 3D electromagnetic-thermal-mechanical coupling finite element model of a gas-insulated bus plug-in connector is studied in [40]. In [37], the effects of the input power on microwave planar devices are studied involving the electro-thermal-mechanical coupling which shows that for moderate input powers the device transfer function can be altered by increasing the losses and frequency shift. In [38], the electromagnetic-thermal characteristics of interconnects are investigated. A set of modified formulas and appropriate thermal models are presented to consider the thermal effects. The computational cost of the multiphysics simulations is very expensive because it involves multiple domains, coupling between domains and often deals with the deformed structure. This problem becomes even more challenging when repetitive multiphysics evaluations are required due to adjustments of the physical geometrical design parameters of the structure. To address this problem, a recent work on multiphysics parametric modeling using the Neuro- 


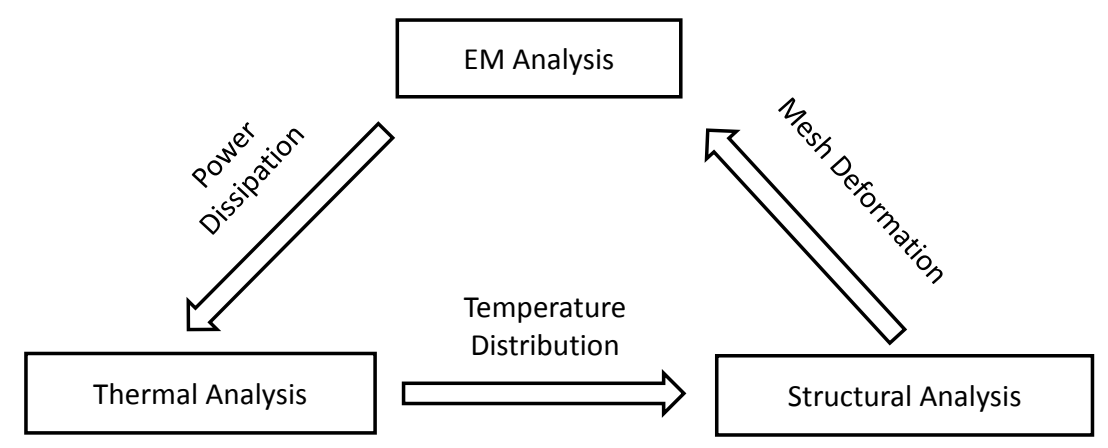

Figure 2.9: The iterative process of multiphysics analysis of the microwave structure. This multiphysics analysis includes three physics domains, EM, thermal, and structure mechanical.

TF modeling method is presented in [116]. The input classification and correlating mapping are introduced to map the multiphysics input parameters onto geometrical input parameters. The parametric model in [116] is much faster than directly using the multiphysics simulator for highly repetitive multiphysics evaluations due to the adjustments of the values of design parameters.

\subsubsection{Description of the EM centric Multiphysics Problem for Microwave Components}

Multiphysics analysis usually involves multiple physics domain analysis such as EM, thermal and structural mechanics [27], [34]. Multiphysics analysis can mimic the behaviors of the EM structures in the real world environment including thermal and other effects. Multiphysics analysis for microwave components is essential for RF designers to gain a better understanding of entire system performance.

To accurately predict the EM behavior in a real world environment, a two- 
way feedback between multiple physics domains is required. Fig. 2.9 shows an illustration of an iterative process of a multiphysics simulation for a microwave structure. S-parameters are the output responses with respect to (w.r.t.) different values of geometrical parameters for the filter example.

In EM single physics (EM only) analysis, i.e., single physics analysis, the Sparameters are independent of the input power. Therefore, for the EM single physics (EM only) analysis, the S-parameter is not affected by the change of the value of the input power. However, during the multiphysics analysis, the input power will be considered by the other physics domains and thereby influence the EM responses. The input power to the filter generates the RF losses in the structure. These RF losses are evaluated using the electric and magnetic fields computed over the entire surface or volume of the device. The RF losses become the heat source which will create the temperature distribution. Thermal analysis is used to calculate the temperature distribution in the structure. Different temperature at different positions in the structure will create the thermal stress and cause the deformation of the structure. Further, the structural analysis is used to calculate the deformation based on the temperature distribution. The resultant structural deformation of the microwave device is looped back to the EM simulator to re-perform meshing and analyze the structure again. Therefore, the S-parameter computation is affected by the input power in the multiphysics environment. This process is repeated iteratively until a steady state final solution is obtained, i.e., until the amount of deformation or changes in temperature between two consecutive iterations are less than a user defined threshold. 
This iterative process takes several iterations to converge to a steady solution. For each iteration we perform the analysis in multiple physics domains and deal with the deformed structure. This makes the multiphysics simulation very timeconsuming and computationally expensive. However, for the EM single physics (EM only) simulation (single physics), it is a one time EM analysis in non-deformed structure, which makes it much cheaper than the multiphysics simulation.

\subsection{Decomposition Techniques}

Decomposition is an important vehicle to solve large-scale problems. The topological decomposition of circuits [121] has been used to address the challenges in large-scale circuit simulations. For EM, structural domain decomposition methods (DDM) [122] - [1] have been used extensively to address EM simulation challenges with complex geometries and large mesh problems. In [123], [1], modular neural networks are investigated for microwave filter design by incorporating a filter equivalent circuit model as the interface for mapping the sub-models. In [124], an automated decomposition approach for the optimization of large microwave systems is introduced with manageable computational CPU time. The overall problem with many design variables is separated into a sequence of sub-problems with a subset of design variables.

\subsection{Conclusion}

In this chapter, a literature review of popularly used EM/multiphysics parametric modeling and design optimization techniques has been discussed. An overview of 
artificial neural network for parametric modeling has been introduced. The structure of artificial neural network and the training methodologies have been reviewed. The knowledge-based neural network has also been discussed as an advanced type of neural networks with prior knowledge as part of internal neural network structure. A novel parametric modeling of EM behavior of microwave components using combined neural networks and pole-residue-based transfer functions is discussed. An overview of space mapping techniques such as input space mapping, implicit space mapping, output space mapping, tuning space mapping, neural space mapping, and recently developed parallel space mapping has also been presented. Further, the EM centric multiphysics simulation has been introduced and the iterative process of multiphysics analysis including EM, thermal, and structure mechanical has been presented. Popular decomposition techniques which are important vehicles to solve large-scale problems has been discussed. 


\section{Chapter 3}

\section{Space Mapping Approach to Electromagnetic Centric Multiphysics Parametric Modeling of Microwave Components}

In this chapter, a novel technique is proposed to develop a low-cost EM centric multi-

physics parametric model for microwave components [34]. In the proposed method, we use SM techniques to combine the computational efficiency of EM single physics (EM only) simulation with the accuracy of the multiphysics simulation. The EM responses with respect to different values of geometrical parameters in non-deformed structures without considering other physics domains are regarded as coarse model. The coarse model is developed using the parametric modeling methods such as artificial neural networks (ANNs) or neuro-transfer function (Neuro-TF) techniques. The EM responses with geometrical and non-geometrical design parameters as variables in the practical deformed structures due to thermal and structural mechanical stress factors are regarded as fine model. The fine model represents the behavior of 
EM centric multiphysics responses. The proposed model includes the EM domain coarse model and two mapping neural networks to map the EM domain (single physics) to the multiphysics domain. Our proposed technique can achieve good accuracy for multiphysics parametric modeling with fewer multiphysics training data and less computational cost.

\subsection{Introduction}

The computational cost of the multiphysics simulations is very expensive because it involves multiple domains, coupling between domains and often deals with the deformed structure. This problem becomes even more challenging when repetitive multiphysics evaluations are required due to adjustments of the physical geometrical design parameters of the structure. To address this problem, a recent work on multiphysics parametric modeling using the Neuro-TF modeling method is presented in [116]. The input classification and correlating mapping are introduced to map the multiphysics input parameters onto geometrical input parameters. The parametric model in [116] is much faster than directly using the multiphysics simulator for highly repetitive multiphysics evaluations due to the adjustments of the values of design parameters.

In this chapter, we propose a new technique which is a significant advance over the work of [116] in an effort to further improve the efficiency of the parametric multiphysics modeling by reducing the number of multiphysics training data. A new space mapping technique is introduced to map the EM domain to the multiphysics domain, as opposed to use direct modeling method in [116]. Our proposed 
technique can work well even when the correlating information needed in [116] is not available. For the first time we elevate the space mapping techniques from solving EM modeling problem to solving the multiphysics modeling problem. We propose to formulate space mapping techniques to build the mapping between the multiphysics domain and EM domain (single physics) considering that EM domain responses are approximate solutions to the EM centric multiphysics responses but much faster than the multiphysics simulations. In our proposed technique, the EM data from EM single physics (EM only) simulation are used to construct a coarse model. The coarse model with geometrical parameters as variables is represented either by ANN model or Neuro-TF model. The fine model represents the behaviors of EM centric multiphysics responses. The inputs of the fine model include the geometrical parameters and non-geometrical parameters. Two mapping modules are proposed to map the EM domain responses to the multiphysics domain responses. One module is the mapping between the multiphysics domain design parameters and EM domain design parameters, and the other module represents the mapping relationship between the non-geometrical design parameters along with frequency parameter of the multiphysics model and the frequency parameter of the coarse model. An adjoint multiphysics model is proposed to guide the gradient-based training and optimization process. Our proposed technique can achieve good accuracy of the EM centric multiphysics model using fewer multiphysics training data compared to the direct parametric modeling method. Thereby the proposed method can reduce the design cycle and increase design efficiency. Once an accurate overall model is developed, it can be used to provide accurate and fast prediction of EM centric multiphysics 
responses with geometrical parameters of microwave components as variables and can be used for higher level design. A tunable four-pole waveguide filter example and an iris coupled microwave cavity filter example are used to demonstrate the efficiency of the proposed parametric modeling technique.

\subsection{Proposed EM centric Multiphysics Paramet- ric Modeling Technique}

In this section, we propose the structure of the multiphysics model which contains the EM domain coarse model and two mapping functions. We propose to formulate the space mapping techniques to establish the relationships between the EM domain coarse model and multiphysics domain fine model. We develop the EM single

physics (EM only) domain coarse model which can be used as the prior knowledge to establish the proposed multiphysics model. We propose the multiphysics model training process w.r.t. different values of geometrical and non-geometrical input parameters and formulate the equations of the adjoint model which can be used to guide the training and optimization process.

\subsubsection{Structure of the Proposed Space Mapped EM Centric Multiphysics Parametric Model}

Here we use a simple fictitious example to illustrate the idea. Fig. 3.1 (a) shows a film capacitor with the length of $10 \mathrm{~mm}$, height of $2 \mathrm{~mm}$ and width $(W)$ of 12 $\mathrm{mm}$. The length and height of the structure are fixed in this example. The relative permittivity is 8 . The width $(W)$ of the capacitor is the geometrical variable in 
Capacitance $4.25 \mathrm{pF}$

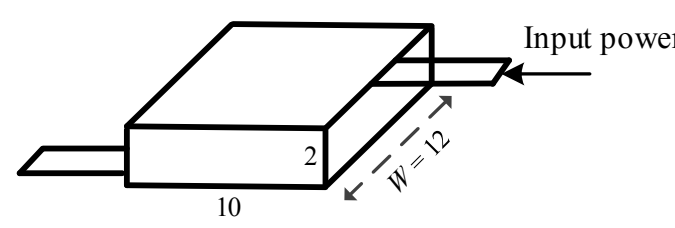

(a)

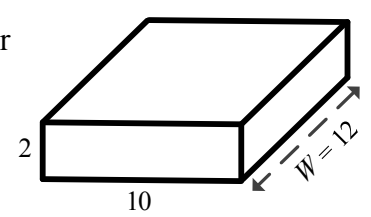

(b)

Capacitance $5.3 \mathrm{pF}$

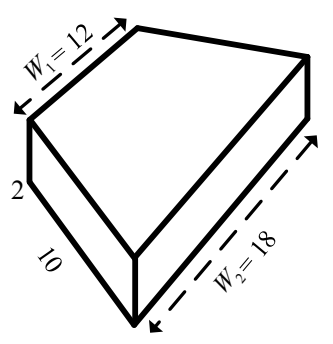

(c)

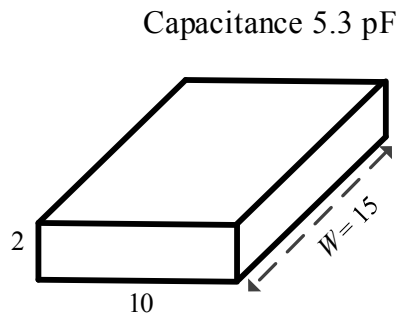

$(d)$

Figure 3.1: Simple illustration of the idea of using space mapping techniques to build the mapping relationship between the multiphysics domain and single physics domain. (a) Original structure of a film capacitor, (b) Structure for electric analysis, i.e., the coarse model, (c) Deformed structure due to high power at one side of the capacitor used for multiphysics analysis, i.e., the fine model and (d) Electric analysis with a different width such that the capacitance of this non-deformed structure (i.e., the mapped coarse model) is the same as the capacitance of deformed structure shown in (c). $W$ is changed from $12 \mathrm{~mm}$ to $15 \mathrm{~mm}$ by mapping.

this example. A high input power is given from the upper plate to the lower plate. Suppose our model output is the capacitance of the structure.

In pure electric analysis, the capacitance is independent of the input power and the capacitance of the device is $4.25 \mathrm{pF}$ shown in Fig. 3.1 (b). While for multiphysics analysis, we include the electrical, thermal, and structural analysis in this example. 


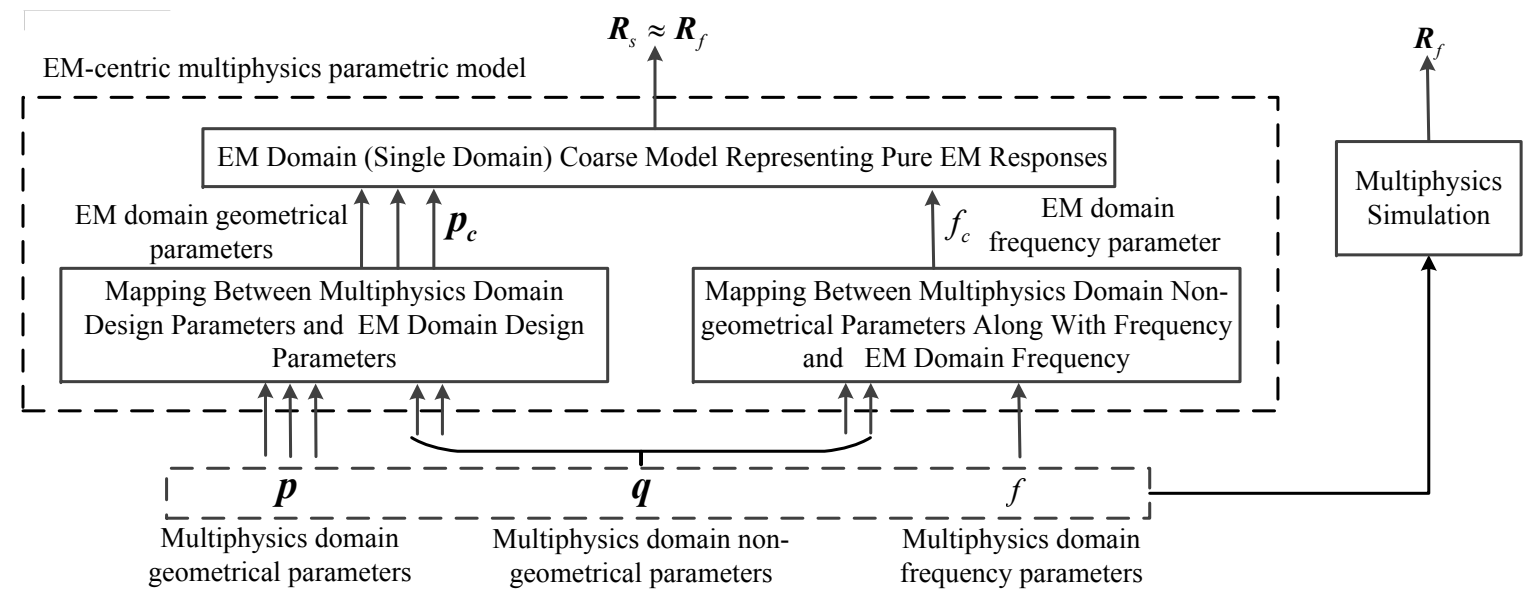

Figure 3.2: Structure of the proposed space mapped multiphysics parametric model exploiting coarse model and space mapping techniques. $\boldsymbol{R}_{s}$ represents the real and imaginary parts of the outputs of the overall multiphysics model (e.g., Sparameters); $\boldsymbol{R}_{f}$ represents the outputs of fine model multiphysics analysis. The first mapping module represents the relationship between the multiphysics domain design parameters and EM domain design parameters. The second mapping module represents space mapping between the non-geometrical input parameters along with frequency parameter of the multiphysics model and the frequency parameter of the EM domain coarse model.

The high input power is considered by the thermal analysis and transformed into the temperature distribution along the device. Here we suppose that temperature is linearly distributed along the length of this device. This temperature distribution becomes the input to the structural analysis. After the structural analysis, the geometrical structure of the device is changed due to the uneven temperature in the structure shown in Fig. 3.1 (c). The capacitance of the deformed structure is changed from $4.25 \mathrm{pF}$ to $5.3 \mathrm{pF}$. The multiphysics simulation is much more expensive than the pure electric (single physics) analysis. When performing the multiphysics analysis, we need to use the entire deformed mesh information to calculate the 
capacitance of the device.

In this example, the coarse model is the non-deformed structure shown in Fig. 3.1 (b), the fine model is the deformed structure shown in Fig. 3.1 (c). The capacitance of the coarse model is not accurate enough to represent the capacitance of the fine model. However, if we change the width $W$ from $12 \mathrm{~mm}$ to $15 \mathrm{~mm}$ shown in Fig. 3.1 (d), the capacitance of this non-deformed structure is the same as the capacitance of the deformed structure shown in Fig. 3.1 (c). Space mapping can be used to map $W$ from $12 \mathrm{~mm}$ to $15 \mathrm{~mm}$. In other words, the EM domain coarse model has been mapped to the multiphysics domain fine model.

In our proposed method, we use the EM single physics (EM only) domain responses in non-deformed structure without considering other physics domains to construct a coarse model. The fine model is the EM responses in the practical deformed structure including thermal, structural mechanic factors. By mapping the coarse model to the fine model, we can get the accurate surrogate model. Let $\boldsymbol{R}_{f}$ represent the vector containing the responses of multiphysics analysis for a microwave component (fine model). Let $\boldsymbol{x}$ represent the design parameters for the multiphysics problem. Let $f$ represent the frequency parameter which is an extra input of the fine model. The task is to construct a surrogate model which is computationally very efficient and also as accurate as the fine model. Let $\boldsymbol{R}_{s}$ represent a response vector of the surrogate model which is required to be

$$
\boldsymbol{R}_{s}(\boldsymbol{x}, f)=\boldsymbol{R}_{f}(\boldsymbol{x}, f)
$$

Here, we propose a multiphysics parametric model (surrogate model) using space 
mapping technique which is illustrated in Fig. 3.2. The surrogate model consists of EM domain based coarse model with geometrical parameters as variables and two space mapping module functions. The EM domain coarse model represents the EM single physics (EM only) behaviors of microwave components which can be used as the prior knowledge to establish the proposed multiphysics model. Two mapping modules are used to map the EM domain responses to the multiphysics domain responses. The first mapping module is trained to represent the relationship between the multiphysics domain design parameters and EM domain design parameters. The second mapping module is developed to represent the mapping between the nongeometrical design parameters along with frequency parameter of the multiphysics model and the frequency parameter of the coarse model. If the coarse model and fine model use the same value of inputs, the output responses will be misaligned. The two proposed mapping modules are used to reduce the misalignment between EM domain (single physics) coarse model and multiphysics domain fine model. After training process, the outputs for the surrogate model w.r.t. different values of geometrical and non-geometrical input parameters can represent the EM responses (e.g, S-parameters) simulated in multiphysics simulator.

To illustrate the proposed multiphysics space mapping technique effectively, we first define the input parameters of the EM domain (single physics) coarse model and multiphysics domain fine model. Let $\boldsymbol{p}$ represent the geometrical parameters of the fine model. The geometrical parameters $\boldsymbol{p}$ are independent of the frequency which is an extra input of the fine model. The design parameters $\boldsymbol{x}$ for the multiphysics problem include not only the geometrical parameters $\boldsymbol{p}$ but also other 
physics domain parameters such as temperature, input power, input voltage and structural stress. Let $\boldsymbol{q}$ represent other physics domain parameters which are considered as non-geometrical design variables. Therefore, the entire input parameters for the multiphysics model are defined as

$$
\boldsymbol{x}=\left[\begin{array}{l}
\boldsymbol{p} \\
\boldsymbol{q}
\end{array}\right]
$$

For the coarse model, let $\boldsymbol{p}_{c}$ represent the geometrical parameters of EM domain and $f_{c}$ represent frequency parameter of EM domain. The input parameters of the EM domain coarse model include only the geometrical parameters, i.e., $\boldsymbol{p}_{c}$ is the inputs to the coarse model. Let $\boldsymbol{R}_{c}$ represent the response vector of the EM domain coarse model as a function of $\boldsymbol{p}_{c}$ and $f_{c}$, defined as $\boldsymbol{R}_{c}\left(\boldsymbol{p}_{c}, f_{c}\right)$.

To correct the changes in the EM responses due to other physics domain parameters, two space mapping module are proposed. The same non-geometrical parameters $\boldsymbol{q}$ are used as inputs for both mapping modules. For the first mapping module, since the relationship between EM domain design parameters and the multiphysics domain design parameters is nonlinear and unknown, we propose to use the neural network to learn this relationship. Let $\boldsymbol{f}_{A N N 1}$ be the neural network mapping function. The multiphysics design parameters containing geometrical parameters $\boldsymbol{p}$ and non-geometrical parameters $\boldsymbol{q}$ are mapped to the geometrical variables $\boldsymbol{p}_{c}$ which are the EM domain design parameters. The mapping function implemented using neural network function is proposed as

$$
\boldsymbol{p}_{c}=\boldsymbol{f}_{A N N 1}\left(\boldsymbol{p}, \boldsymbol{q}, \boldsymbol{w}_{1}\right)
$$


where $\boldsymbol{p}$ and $\boldsymbol{q}$ are the inputs to the neural network, $\boldsymbol{p}_{c}$ is the output of the neural network and $\boldsymbol{w}_{1}$ represents a vector containing all the weight parameters of this mapping neural network.

Similarly, for the second mapping module, since the relationship between the frequency parameter of the coarse model and the non-geometrical input parameters along with frequency parameter of the multiphysics model is nonlinear and unknown, we propose to use the second neural network to learn this relationship. Let $f_{A N N 2}$ be the mapping function. The frequency parameter $f$ and non-geometrical parameters $\boldsymbol{q}$ of the multiphysics model are mapped directly to the input frequency $f_{c}$ of the EM domain (single physics) based coarse model. The frequency mapping function is proposed as

$$
f_{c}=f_{A N N 2}\left(\boldsymbol{q}, f, \boldsymbol{w}_{2}\right)
$$

where $\boldsymbol{q}$ and $f$ are the inputs to the neural network, $f_{c}$ is the output of the neural network and $\boldsymbol{w}_{2}$ represents a vector containing all the weight parameters in this frequency mapping network. The responses of the proposed model with geometrical and non-geometrical parameters as variables are defined as

$$
\begin{aligned}
& \boldsymbol{R}_{s}\left(\boldsymbol{p}, \boldsymbol{q}, f, \boldsymbol{w}_{1}^{*}, \boldsymbol{w}_{2}^{*}\right)= \\
& \quad \boldsymbol{R}_{c}\left(\boldsymbol{f}_{A N N 1}\left(\boldsymbol{p}, \boldsymbol{q}, \boldsymbol{w}_{1}^{*}\right), f_{A N N 2}\left(f, \boldsymbol{q}, \boldsymbol{w}_{2}^{*}\right)\right)
\end{aligned}
$$

where $\boldsymbol{w}_{1}^{*}$ and $\boldsymbol{w}_{2}^{*}$ are the solutions from the following optimization problem

$$
\min _{\left[\boldsymbol{w}_{1}, \boldsymbol{w}_{2}\right]} \sum_{j \in T_{r}} \sum_{l \in \Omega}\left\|\boldsymbol{R}_{s}\left(\boldsymbol{p}_{j}, \boldsymbol{q}_{j}, f_{l}, \boldsymbol{w}_{1}, \boldsymbol{w}_{2}\right)-\boldsymbol{d}_{j, l}\right\|
$$

where $\Omega$ represents the index set of the frequency samples. $T_{r}$ represents the index 
set of training samples, i.e., $T_{r}=\left\{1,2, \ldots, n_{s}\right\}$, where $n_{s}$ is the total number of the multiphysics training data. $\boldsymbol{d}$ represents the data from multiphysics simulation w.r.t. different values of geometrical and non-geometrical design parameters. $\boldsymbol{d}_{j, l}$ represents the multiphysics data from the $j$ th training sample at the $l$ th frequency sample. The parameters $\boldsymbol{w}_{1}$ and $\boldsymbol{w}_{2}$ are trained to make the outputs of the proposed model match the multiphysics data at each frequency and each geometrical sample. Since we use many EM single physics (EM only) data to build the coarse model, the total number of multiphysics training samples $n_{s}$ is relatively smaller than that in the direct methods which use the multiphysics training data to build the multiphysics model. Therefore, the proposed multiphysics model can be developed with relatively fewer multiphysics training data and less computational cost.

\subsubsection{EM Domain Coarse Model Construction}

To develop the proposed multiphysics parametric model using space mapping technique, the first step is to build the EM domain (single physics) coarse model w.r.t. different values of geometrical parameters. The output responses of the EM sin-

gle physics (EM only) domain (single physics) simulation can be considered as the available knowledge for the computationally expensive multiphysics simulation. The relatively inexpensive EM simulation data from the EM domain analysis can be used to construct an EM domain coarse model. The expensive multiphysics simulation can be replaced by the relatively inexpensive EM domain simulation.

The first step for constructing the EM single physics (EM only) based coarse model is data generation. To generate the EM domain training data, we need to 
first classify the multiphysics input parameters into three sets of parameters, the geometrical parameters $\boldsymbol{p}$, the frequency parameter $f$ and other physics domain non-geometrical parameters $\boldsymbol{q}$. Once the multiphysics parameter classification is finished, we can determine the variables of the inputs $\boldsymbol{p}_{c}$ which contain the same geometrical variables as the overall model geometrical inputs $\boldsymbol{p}$. To guarantee the accuracy of the overall multiphysics model, the ranges of geometrical parameters for the EM domain (single physics) coarse model are selected to be slightly larger than those in the overall multiphysics model.

After data generation, an EM domain coarse model with geometrical parameters as variables is developed using the parametric modeling methods such as artificial neural network (ANN) modeling method or neuro-transfer function (Neuro-TF) technique. Pure ANN is a simpler technique to learn EM behavior without having to rely on the complicated internal details of passive components. Neuro-TF technique is more efficient when the frequency responses have sharp resonances. After training process, the trained EM domain (single physics) coarse model can be used to represent the behavior of EM single physics (EM only) responses and is ready to be used as a prior knowledge for the overall multiphysics model development.

\subsubsection{Proposed Space Mapped EM Centric Multiphysics Parametric Model Training Process}

To develop an accurate space mapped multiphysics parametric model, we propose to perform a two stage training process. The first stage is the EM domain (single physics) coarse model training which is defined in Section 3.2.2. After the EM 
domain coarse model is trained, the parameters in the coarse model are fixed. We can construct the proposed multiphysics parametric model w.r.t. different values of geometrical and non-geometrical input parameters. The second stage is the multiphysics domain model training. We use design of experiments (DOE) sampling method [125] to generate the multiphysics data. We perform multiphysics simulations to generate multiphysics training data for the proposed surrogate model. We first perform the unit mapping for the two mapping networks by setting the values of EM domain inputs to be equal to the values of multiphysics inputs. The purpose of the unit mapping is to provide good initial values for the mapping neural networks before training them. After the unit mappings are established, the overall multiphysics model training process is performed to obtain the final surrogate model. The training data for this step are the samples with the multiphysics input parameters as the model input data and EM responses by multiphysics analysis as the target data for model outputs. During this stage, we optimize the weight parameters $\boldsymbol{w}_{1}$ and $\boldsymbol{w}_{2}$ of the two mapping modules to reduce the misalignment between the proposed multiphysics model and the multiphysics training data.

During the proposed multiphysics parametric model training process, the firstorder derivatives $\partial \boldsymbol{R}_{s}^{T} / \partial \boldsymbol{w}_{1}$ and $\partial \boldsymbol{R}_{s}^{T} / \partial \boldsymbol{w}_{2}$ are required to guide the gradient-based training process. In order to get the derivative information for the weighting parameters $\boldsymbol{w}_{1}$ and $\boldsymbol{w}_{2}$, we need the derivative information of $\partial \boldsymbol{R}_{c}^{T} / \partial \boldsymbol{p}_{c}$ and $\partial \boldsymbol{R}_{c}^{T} / \partial f_{c}$. For this purpose, we propose to establish an adjoint multiphysics model. Once the adjoint model is developed, the outputs of the adjoint model will provide the first order derivative to guide the gradient-based training process. The adjoint multiphysics 
model consists of the adjoint EM domain coarse model and two adjoint neural network models [4]. Since the coarse model is represented by either the ANN model or the Neuro-TF model, the adjoint EM domain coarse model is represented by either the adjoint neural network [4] or adjoint Neuro-TF model [126]. After the neural network model is trained, the weighting parameters are fixed. We create an adjoint neural network [4] based on a similar structure with the same weighting parameters as the trained neural network, i.e., the adjoint model. Let $\boldsymbol{G}_{E M}$ represent the derivative information of the EM domain outputs w.r.t. the EM domain design parameters. Let $\boldsymbol{F}_{E M}$ represent the derivative information of the EM domain outputs w.r.t. the EM domain frequency parameter [4]. Let $\boldsymbol{G}_{M P}$ and $\boldsymbol{M}_{M P}$ be the outputs of the adjoint model of the first mapping function w.r.t. the variables $\boldsymbol{p}, \boldsymbol{q}$ and $f$. More specifically, $\boldsymbol{G}_{M P}$ represents the derivative information of the EM domain design parameters w.r.t. the multiphysics domain geometrical parameters. $\boldsymbol{M}_{M P}$ represents the derivative information of the EM domain design parameters w.r.t. the multiphysics domain non-geometrical parameters. Let $\boldsymbol{F}_{M P}$ be the outputs of the adjoint model of the second mapping function. $\boldsymbol{F}_{M P}$ represents the derivative information of the EM domain frequency parameter w.r.t. the multiphysics domain non-geometrical parameters. The proposed adjoint multiphysics model is shown in Fig. 3.3.

During the overall model training process, the neural network internal parameters $\boldsymbol{w}_{1}$ and $\boldsymbol{w}_{2}$ are the optimization variables. The first order derivatives of the overall multiphysics model output $\boldsymbol{R}_{s}$ w.r.t the neural network internal parameters are required for training technique. The derivatives of the $\partial \boldsymbol{R}_{c}^{T}\left(\boldsymbol{p}_{c}, f_{c}\right) / \partial \boldsymbol{p}_{c}$ 


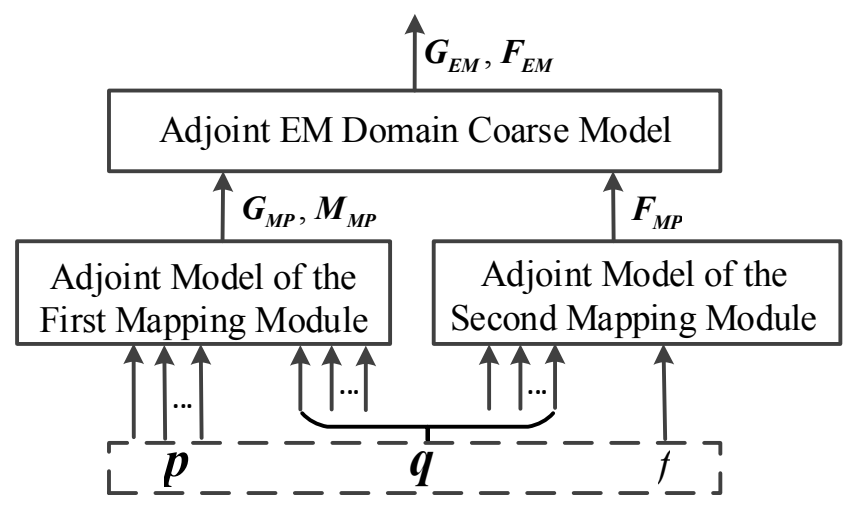

Figure 3.3: Structure of the proposed adjoint multiphysics model including the adjoint EM domain coarse model and two adjoint neural network models. The adjoint model of the first mapping module is the adjoint neural network of $f_{A N N 1}$ and the adjoint model of the second mapping module is the adjoint neural network of $f_{A N N 2}$. The purpose of this adjoint model is to privide the derivative information to guide the training and optimization process.

and $\partial \boldsymbol{R}_{c}^{T}\left(\boldsymbol{p}_{c}, f_{c}\right) / \partial f_{c}$ can be obtained from the outputs of the adjoint multiphysics model shown as

$$
\begin{aligned}
& \frac{\partial \boldsymbol{R}_{c}^{T}\left(\boldsymbol{p}_{c}, f_{c}\right)}{\partial \boldsymbol{p}_{c}}=\boldsymbol{G}_{E M} \\
& \frac{\partial \boldsymbol{R}_{c}^{T}\left(\boldsymbol{p}_{c}, f_{c}\right)}{\partial f_{c}}=\boldsymbol{F}_{E M}
\end{aligned}
$$

The first order derivatives of the overall multiphysics model output $\boldsymbol{R}_{s}$ w.r.t weight parameters $\boldsymbol{w}_{1}$ of the first mapping module are formulated by

$$
\begin{aligned}
& \frac{\partial \boldsymbol{R}_{s}^{T}\left(\boldsymbol{p}, \boldsymbol{q}, f, \boldsymbol{w}_{1}, \boldsymbol{w}_{2}\right)}{\partial \boldsymbol{w}_{1}} \\
& =\frac{\partial \boldsymbol{p}_{c}^{T}\left(\boldsymbol{p}, \boldsymbol{q}, \boldsymbol{w}_{1}\right)}{\partial \boldsymbol{w}_{1}} \frac{\partial \boldsymbol{R}_{c}^{T}\left(\boldsymbol{p}_{c}, f_{c}\right)}{\partial \boldsymbol{p}_{c}} \\
& =\frac{\partial \boldsymbol{p}_{c}^{T}\left(\boldsymbol{p}, \boldsymbol{q}, \boldsymbol{w}_{1}\right)}{\partial \boldsymbol{w}_{1}} \cdot \boldsymbol{G}_{E M}
\end{aligned}
$$

where $\boldsymbol{G}_{E M}$ is the output of the adjoint EM domain coarse model. $\partial \boldsymbol{p}_{c}^{T}\left(\boldsymbol{p}, \boldsymbol{q}, \boldsymbol{w}_{1}\right) / \partial \boldsymbol{w}_{1}$ 
represents the derivative information of EM domain design parameters w.r.t. the weighting parameters of the first mapping function $\boldsymbol{f}_{A N N 1}$ calculated by the back propagation [16]. Similarly, the first order derivatives of the overall multiphysics model output $\boldsymbol{R}_{s}$ w.r.t weight parameters $\boldsymbol{w}_{2}$ of the second mapping module are derived by

$$
\begin{aligned}
& \frac{\partial \boldsymbol{R}_{s}^{T}\left(\boldsymbol{p}, \boldsymbol{q}, f, \boldsymbol{w}_{1}, \boldsymbol{w}_{2}\right)}{\partial \boldsymbol{w}_{2}} \\
& =\frac{\partial f_{c}\left(\boldsymbol{q}, f, \boldsymbol{w}_{2}\right)}{\partial \boldsymbol{w}_{2}} \frac{\partial \boldsymbol{R}_{c}^{T}\left(\boldsymbol{p}_{c}, f_{c}\right)}{\partial f_{c}} \\
& =\frac{\partial f_{c}\left(\boldsymbol{q}, f, \boldsymbol{w}_{2}\right)}{\partial \boldsymbol{w}_{2}} \cdot \boldsymbol{F}_{E M}
\end{aligned}
$$

where $\boldsymbol{F}_{E M}$ is the output of the adjoint EM domain coarse model. $\partial f_{c}\left(\boldsymbol{q}, f, \boldsymbol{w}_{2}\right) / \partial \boldsymbol{w}_{2}$ represents the derivative information of EM domain mapped frequency w.r.t. the weighting parameters of the second mapping function $f_{A N N 2}$ calculated by the back propagation [16].

The detailed multiphysics training mechanism of the overall multiphysics parametric model exploiting space mapping technique is illustrated in Fig. 3.4. The overall multiphysics model training process is performed by adjusting the neural network weights of the two mapping modules to minimize the training error between the proposed model and multiphysics data, formulated as

$$
\begin{aligned}
& E_{T r}\left(\boldsymbol{w}_{1}, \boldsymbol{w}_{2}\right)= \\
& \frac{1}{2 n_{s}} \sum_{j \in T_{r}} \sum_{l \in \Omega}\left\|\boldsymbol{R}_{s}\left(\boldsymbol{p}_{j}, \boldsymbol{q}_{j}, f_{l}, \boldsymbol{w}_{1}, \boldsymbol{w}_{2}\right)-\boldsymbol{d}_{j, l}\right\|^{2}
\end{aligned}
$$

After training, an independent set of multiphysics testing data are used to test the trained overall multiphysics parametric model. If the testing error $E_{T e}$ is lower 


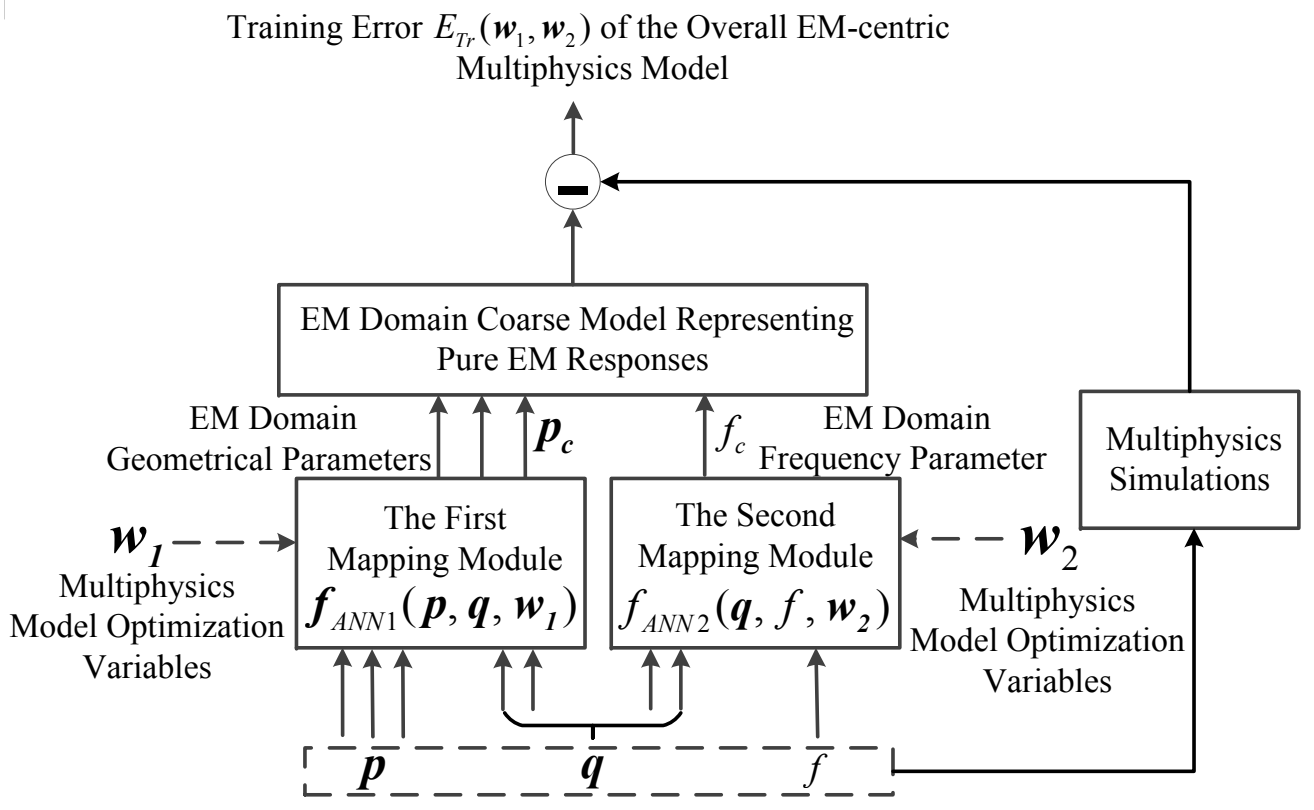

Figure 3.4: The detailed training mechanism of the overall multiphysics parametric model exploiting space mapping technique. The objective is to minimize the training error between the proposed model and multiphysics data. The variables of this training process are the weighting parameters $\boldsymbol{w}_{1}$ and $\boldsymbol{w}_{2}$ of the two mapping modules between the multiphysics domain and the single physics domain.

than a user define threshold $\varepsilon$, the training process terminates and the overall model has been developed. Otherwise, the overall multiphysics model training process will be repeated by adjusting the numbers of hidden neurons in the neural networks. A flowchart of the proposed multiphysics model development process is shown in Fig. 3.5. After the overall multiphysics parametric model is developed, it is ready to be used for higher level multiphysics design optimization. 


\subsubsection{Use of Proposed Model for Multiphysics Design Op- timization}

After training is finished, the proposed multiphysics model can be used for multiphysics design optimization. During the optimization process, our proposed adjoint multiphysics model can be used to obtain the first order derivative information of the overall model output $\boldsymbol{R}_{s}$ w.r.t the overall model inputs $\boldsymbol{p}$ and $\boldsymbol{q}$ to guide the gradient-based design optimization.

In order to get the derivative information of $\partial \boldsymbol{R}_{s}^{T} / \partial \boldsymbol{p}$ and $\partial \boldsymbol{R}_{s}^{T} / \partial \boldsymbol{q}$, we need to evaluate derivatives throughout various parts of the model. The derivatives of the $\partial \boldsymbol{p}_{c}^{T}\left(\boldsymbol{p}, \boldsymbol{q}, \boldsymbol{w}_{1}\right) / \partial \boldsymbol{p}$ and $\partial \boldsymbol{p}_{c}^{T}\left(\boldsymbol{p}, \boldsymbol{q}, \boldsymbol{w}_{1}\right) / \partial \boldsymbol{q}$ can be obtained directly from the adjoint model of the first mapping model, formulated as

$$
\begin{gathered}
\frac{\partial \boldsymbol{p}_{c}^{T}\left(\boldsymbol{p}, \boldsymbol{q}, \boldsymbol{w}_{1}\right)}{\partial \boldsymbol{p}}=\boldsymbol{G}_{M P} \\
\frac{\partial \boldsymbol{p}_{c}^{T}\left(\boldsymbol{p}, \boldsymbol{q}, \boldsymbol{w}_{1}\right)}{\partial \boldsymbol{q}}=\boldsymbol{M}_{M P}
\end{gathered}
$$

Similarly, the derivative of the $\partial f_{c}\left(\boldsymbol{q}, f, \boldsymbol{w}_{2}\right) / \partial \boldsymbol{q}$ can be obtained directly from the adjoint model of the second mapping model, formulated as

$$
\frac{\partial f_{c}\left(\boldsymbol{q}, f, \boldsymbol{w}_{2}\right)}{\partial \boldsymbol{q}}=\boldsymbol{F}_{M P}
$$

Based on the proposed adjoint multiphysics model, the detailed derivative for- 


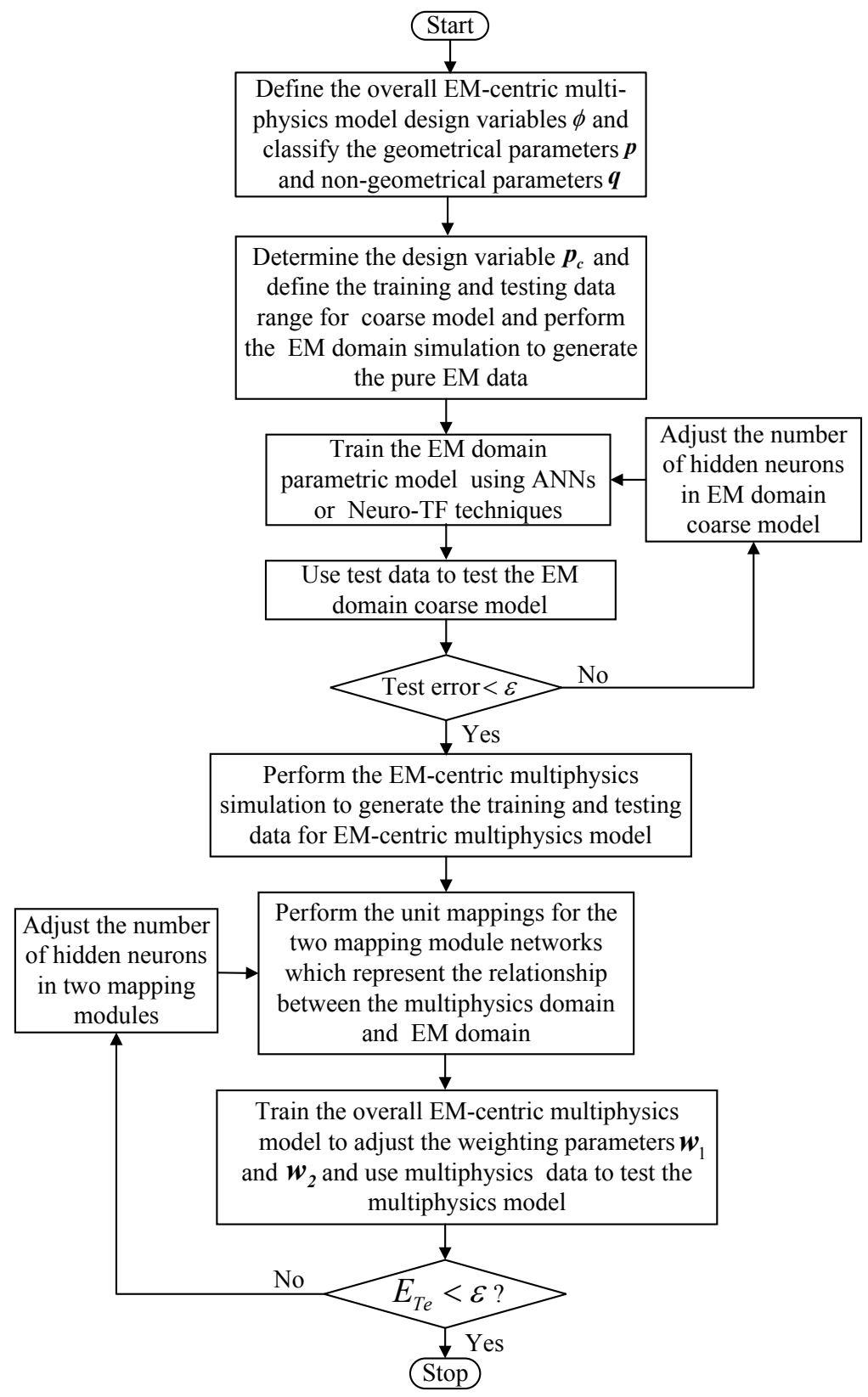

Figure 3.5: The flowchart of the development process of the multiphysics parametric model exploiting the EM domain coarse model and space mapping between the multiphysics domain and the single physics EM domain. 
mula of $\boldsymbol{R}_{s}$ w.r.t $\boldsymbol{p}$ which is used to guide the optimization process is derived as

$$
\begin{aligned}
& \frac{\partial \boldsymbol{R}_{s}^{T}(\boldsymbol{p}, \boldsymbol{q}, f)}{\partial \boldsymbol{p}} \\
& =\frac{\partial \boldsymbol{p}_{c}^{T}(\boldsymbol{p}, \boldsymbol{q})}{\partial \boldsymbol{p}} \frac{\partial \boldsymbol{R}_{c}^{T}\left(\boldsymbol{p}_{c}, f_{c}\right)}{\partial \boldsymbol{p}_{c}} \\
& =\boldsymbol{G}_{M P} \cdot \boldsymbol{G}_{E M}
\end{aligned}
$$

where $\boldsymbol{G}_{M P}$ is the output of the adjoint model of the first mapping function and $\boldsymbol{G}_{E M}$ is the output of the adjoint EM domain coarse model. When calculating the derivative information of $\boldsymbol{R}_{s}$ w.r.t $\boldsymbol{q}$, since the non-geometrical parameters $\boldsymbol{q}$ are the inputs of both of the two mapping module networks, the detailed derivative formula is derived as

$$
\begin{aligned}
\frac{\partial \boldsymbol{R}_{s}^{T}(\boldsymbol{p}, \boldsymbol{q}, f)}{\partial \boldsymbol{q}} & =\frac{\partial \boldsymbol{p}_{c}^{T}(\boldsymbol{q}, f)}{\partial \boldsymbol{q}} \frac{\partial \boldsymbol{R}_{c}^{T}\left(\boldsymbol{p}_{c}, f_{c}\right)}{\partial \boldsymbol{p}_{c}} \\
& +\frac{\partial f_{c}(\boldsymbol{q}, f)}{\partial \boldsymbol{q}} \frac{\partial \boldsymbol{R}_{c}^{T}\left(\boldsymbol{p}_{c}, f_{c}\right)}{\partial f_{c}} \\
& =\boldsymbol{M}_{M P} \cdot \boldsymbol{G}_{E M}+\boldsymbol{F}_{M P} \cdot \boldsymbol{F}_{E M}
\end{aligned}
$$

where $\boldsymbol{M}_{M P}$ is the output of the adjoint model of the first mapping function. $\boldsymbol{F}_{M P}$ is the output of the adjoint model of the second mapping function. $\boldsymbol{G}_{E M}$ and $\boldsymbol{F}_{E M}$ are the outputs of the adjoint EM domain coarse model. The derivatives calculated in Equation (15) and (16) are thus used to guide the gradient-based design optimization with the developed multiphysics parametric model. 


\subsection{Numerical Examples}

\subsubsection{Multiphysics Parametric Modeling of Tunable Four- Pole Waveguide Filter Using Piezo Actuator}

The first example under consideration is a four-pole wave-guide filter [127] with tuning elements as the posts of the square cross section placed at the center of each cavity and each coupling window. The piezo actuator will have a geometric strain proportional to an applied electric field through the piezoelectric effect [128]. The material for the piezo actuator is Lead Zirconate Titanate (PZT-5H). It is z-polarized and generates mainly z-directional deflection of the device. In this example, piezo actuators are used to control the size of a small air gap between the top of posts and the bottom side of the piezo actuators which provide the tunability for wave-guide filter, shown in Fig. 3.6, where height $\left(h_{1}\right)$ and height $\left(h_{2}\right)$ are the heights of the tuning posts in the coupling windows. Height $\left(h_{c 1}\right)$ and height $\left(h_{c 2}\right)$ are the heights of the square cross section placed in the center of the resonator cavities. Voltages $\left(V_{1}\right)$ and $\left(V_{2}\right)$ are the electronic potentials that are applied across the piezo actuator, which will cause the deformation of the piezo actuator and further change the frequency responses of the device. The input and output waveguides, as well as the resonant cavities, are standard WR-75 waveguides (width $=19.050 \mathrm{~mm}$, height $=9.525 \mathrm{~mm})[127]$. The length of the structure is 77.6 $\mathrm{mm}$. The thickness of all the coupling windows is set to $2 \mathrm{~mm}$. Frequency $f$ is an additional input. The design parameter for this example has six variables, i.e., $\boldsymbol{x}=\left[\begin{array}{llllll}h_{1} & h_{2} & h_{c 1} & h_{c 2} & V_{1} & V_{2}\end{array}\right]^{T}$. The geometrical input variables to the overall multiphysics model are $\boldsymbol{p}=\left[\begin{array}{llll}h_{1} & h_{2} & h_{c 1} & h_{c 2}\end{array}\right]^{T}$. The non-geometrical input variables to the 


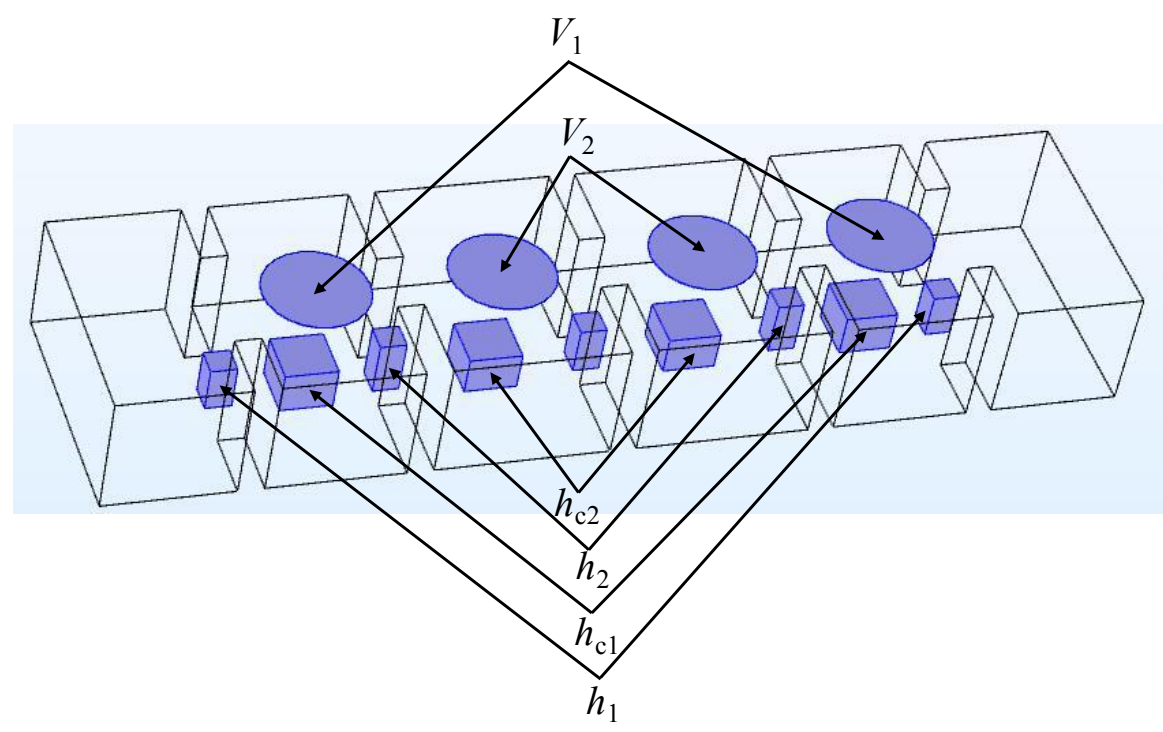

Figure 3.6: Structure of the four-pole waveguide filter using piezo actuator with multiphysics model design variables $\boldsymbol{x}=\left[\begin{array}{llllll}h_{1} & h_{2} & h_{c 1} & h_{c 2} & V_{1} & V_{2}\end{array}\right]^{T}$. The input and output waveguides, as well as the resonant cavities, are standard WR-75 waveguides (width $=19.050 \mathrm{~mm}$, height $=9.525 \mathrm{~mm}$ ). The length of the structure is $77.6 \mathrm{~mm}$. The thickness of all the coupling windows is set to $2 \mathrm{~mm}$.

overall multiphysics model are $\boldsymbol{q}=\left[\begin{array}{ll}V_{1} & V_{2}\end{array}\right]^{T}$ which are the tuning variables. The model has two outputs, i.e., $\boldsymbol{y}=\left[R S_{11} I S_{11}\right]^{T}$, which are the real and imaginary parts of the overall multiphysics model output $S_{11}$ w.r.t. different values of geometrical and non-geometrical input parameters. For the coarse model construction, we consider only the EM single physics (EM only) simulation. The coarse model has four design variables $\boldsymbol{p}_{c}=\left[\begin{array}{llll}h_{1} & h_{2} & h_{c 1} & h_{c 2}\end{array}\right]^{T}$. Frequency $f_{c}$ is an additional input of the EM domain (single physics) coarse model.

COMSOL MULTIPHYSICS 5.2 is used to perform the multiphysics simulation to generate the overall multiphysics model training and testing data, w.r.t. differ- 
ent geometrical and non-geometrical input parameters. The actual process of this multiphysics problem is shown in Fig. 8. The fine model actually uses the entire mesh information to calculate the multiphysics responses while our technique uses the mapping functions to represent the output response changes caused by other physics domains. Fig. 3.8 shows the deformation information of the cavity filter

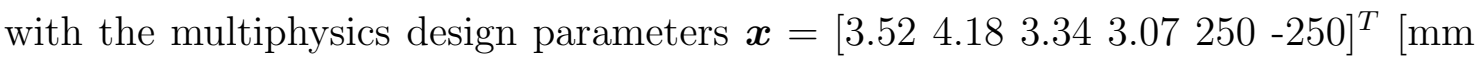
$\mathrm{mm} \mathrm{mm} \mathrm{mm} \mathrm{V}$. We can see that with the positive voltage the piezo actuator deflects toward the bottom while with negative voltage the piezo actuator deflects upwards the bottom. These deformations make the outputs of the multiphysics simulation different from the outputs of the EM single physics (EM only) simulation. Fig. 3.9 shows the output responses using EM domain (single physics) simulation and multiphysics simulation for this cavity filter example, i.e., the coarse model response and overall model response using the same geometrical parameters. From the figure we can see the single physics analysis is not accurate enough to represent the multiphysics responses. Our multiphysics model is more accurate because we include other physics domain besides the EM domain effects into our model. For EM domain (single physics) coarse model data generation w.r.t. different geometrical input parameters, the EM single physics (EM only) evaluation is performed by ANSYS HFSS EM simulator using the fast simulation feature. Design of experiments (DOE) method is used as the sampling method for both EM domain (single physics) coarse model and multiphysics domain overall model data generation.

The EM single physics (EM only) simulation data with geometrical parameters as variables used to construct the EM domain (single physics) coarse model uses nine 


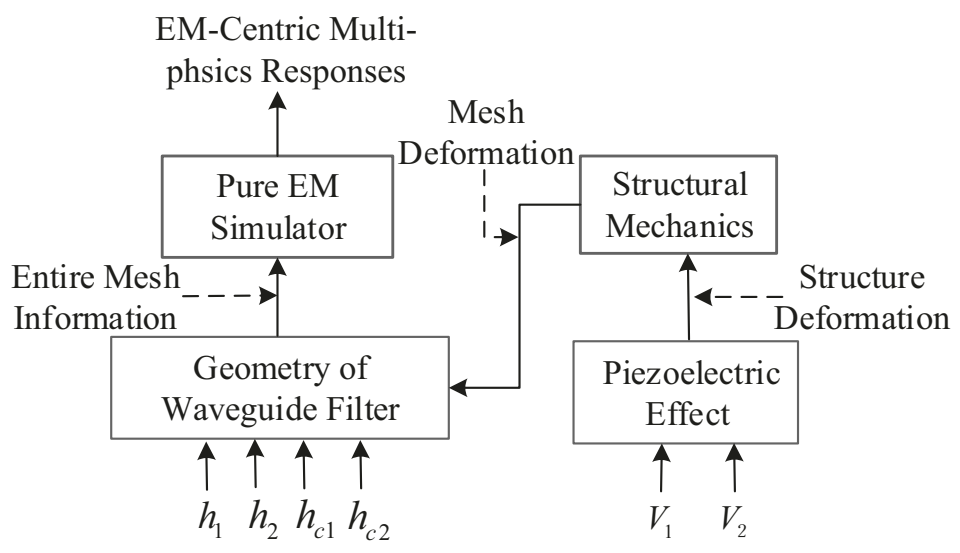

Figure 3.7: The actual process of the multiphysics problem for the four-pole waveguide filter example using the COMSOL software.

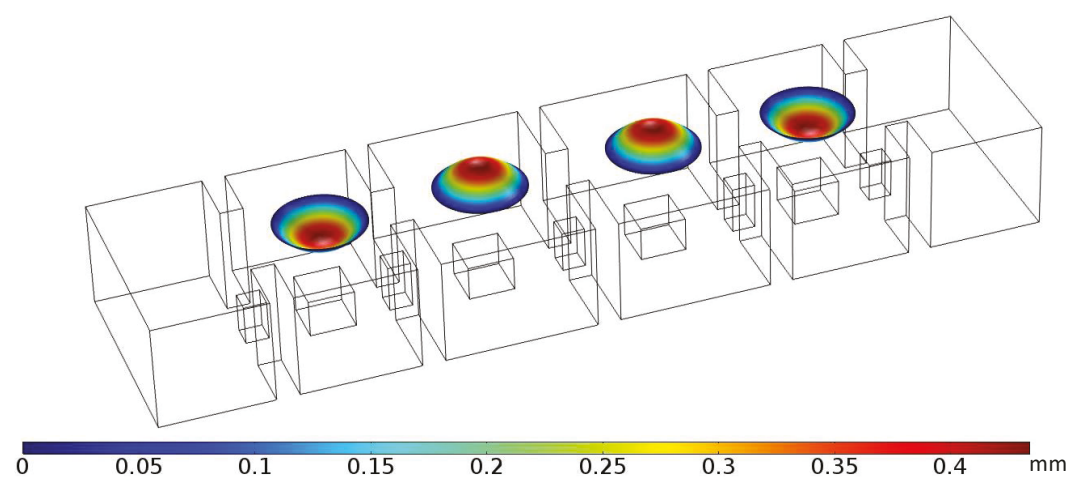

Figure 3.8: The structural deformation in the four-pole waveguide filter caused by the input voltages.

levels of DOE for defining samples of the training data, i.e., a total of 81 samples of EM domain (single physics) training data, and eight levels of DOE for defining samples of the testing data, i.e., a total of 64 samples of testing data. While for the overall multiphysics model data, we only use five levels of DOE for defining samples of the training data, i.e., a total of 25 samples of multiphysics training data. The input ranges of geometrical parameters for the EM domain (single physics) coarse 


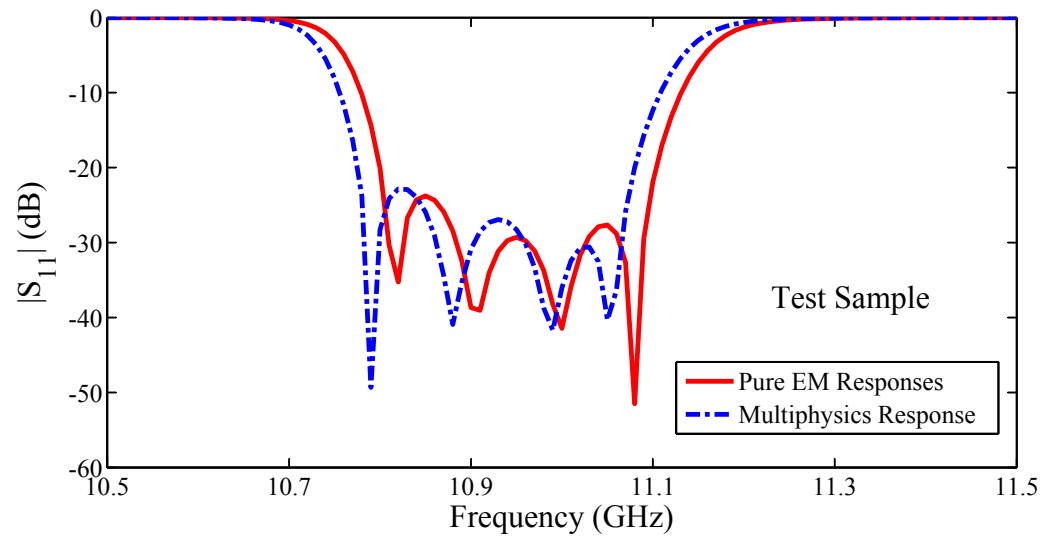

Figure 3.9: Comparison of the magnitude in decibels of $S_{11}$ of the EM single physics (EM only) responses and multiphysics analysis responses using the same geometrical parameters for the four-pole waveguide filter. From the figure we can see that without mapping, the single physics analysis is not accurate enough to represent the multiphysics responses.

model should be larger than the overall multiphysics model to accommodate the mapping between the EM domain and multiphysics domain. The physical shape of the training and testing structure for this example is shown in Fig. 3.6 and the specific values of training data and testing data for both EM domain (single physics) coarse model and multiphysics domain surrogate model are shown in Table 3.1. The testing data are randomly selected within the training ranges and never used in the training process. The frequency range for model development is from $10.5 \mathrm{GHz}$ to $11.5 \mathrm{GHz}$.

For this example, the pole-residue-based Neuro-TF technique is used to construct the EM domain coarse model with geometrical parameters as variables. The number of hidden neurons of the neural networks that represent the relationships between the geometrical parameters and poles/residues is 10. The EM domain 
Table 3.1: Definition of Training and Testing Data for EM Domain (Single Physics) Coarse Model and Multiphysics Domain Overall Model for the Four-Pole Waveguide Filter Example

\begin{tabular}{|c|c|c|c|c|c|c|c|}
\hline \multirow{2}{*}{\multicolumn{2}{|c|}{$\begin{array}{l}\text { Input Variables } \\
\text { to the Model }\end{array}$}} & \multicolumn{3}{|c|}{ Training Data Range } & \multicolumn{3}{|c|}{ Testing Data Range } \\
\hline & & \multirow{2}{*}{$\begin{array}{l}\text { Min } \\
3.42\end{array}$} & \multirow{2}{*}{$\begin{array}{l}\text { Max } \\
3.62\end{array}$} & \multirow{2}{*}{$\begin{array}{l}\text { Step } \\
0.025\end{array}$} & \multirow{2}{*}{$\frac{\text { Min }}{3.4325}$} & \multirow{2}{*}{$\frac{\operatorname{Max}}{3.6075}$} & \multirow{2}{*}{$\begin{array}{l}\text { Step } \\
0.025\end{array}$} \\
\hline EM Data & $\begin{array}{c}h_{1} \\
(\mathrm{~mm})\end{array}$ & & & & & & \\
\hline \multirow[t]{3}{*}{$\begin{array}{l}\text { (Coarse } \\
\text { Model) }\end{array}$} & $\begin{array}{c}h_{2} \\
(\mathrm{~mm})\end{array}$ & 4.08 & 4.28 & 0.025 & 4.0925 & 4.2675 & 0.025 \\
\hline & $\begin{array}{c}h_{c 1} \\
(\mathrm{~mm})\end{array}$ & 3.18 & 3.38 & 0.025 & 3.1925 & 3.3675 & 0.025 \\
\hline & $\begin{array}{c}h_{c 2} \\
(\mathrm{~mm})\end{array}$ & 2.94 & 3.14 & 0.025 & 2.9525 & 3.1275 & 0.025 \\
\hline \multirow{6}{*}{$\begin{array}{l}\text { Multi- } \\
\text { physics } \\
\text { Data } \\
\text { (Overall } \\
\text { Model) }\end{array}$} & $\begin{array}{c}h_{1} \\
(\mathrm{~mm})\end{array}$ & 3.44 & 3.60 & 0.04 & 3.45 & 3.59 & 0.02 \\
\hline & $\begin{array}{c}h_{2} \\
(\mathrm{~mm}) \\
\end{array}$ & 4.10 & 4.26 & 0.04 & 4.11 & 4.25 & 0.02 \\
\hline & $\begin{array}{c}h_{c 1} \\
(\mathrm{~mm})\end{array}$ & 3.22 & 3.34 & 0.03 & 3.2275 & 3.3325 & 0.015 \\
\hline & $\begin{array}{c}h_{c 2} \\
(\mathrm{~mm})\end{array}$ & 2.98 & 3.10 & 0.03 & 2.9875 & 3.0925 & 0.015 \\
\hline & $\begin{array}{c}V_{1} \\
(\mathrm{~V})\end{array}$ & -400 & 400 & 200 & -350 & 350 & 100 \\
\hline & $\begin{array}{c}V_{2} \\
(\mathrm{~V})\end{array}$ & -400 & 400 & 200 & -350 & 350 & 100 \\
\hline
\end{tabular}

(single physics) coarse model using the Neuro-TF techniques is trained using the NeuroModelerPlus software. The average training error for the EM domain coarse model development is $1.11 \%$, while the average testing error is $1.38 \%$. After an 
accurate EM domain coarse model is developed, we can continue to set up the proposed multiphysics model which can accurately represent the multiphysics data with different values of geometrical and non-geometrical design parameters as variables. The overall model including the Neuro-TF coarse model and two mapping neural networks is also constructed and trained using the NeuroModelerPlus software which as shown in Fig. 3.7. Numbers of hidden neurons for the two mapping neural network modules are 4 and 2 respectively. The average training error for the multiphysics domain overall model development is $1.56 \%$, while the average testing error is $1.63 \%$. The overall multiphysics model training process takes about 10 minutes including the parameter extraction, EM domain coarse model construction and overall multiphysics domain model development.

For comparison purpose, ANN model (i.e., without mapping) is directly trained to learn multiphysics data for two cases, case 1 being with fewer multiphysics training data (25 sets of data) and case 2 being with more multiphysics training data (81 sets of data). We also train the model using the Neuro-TF modeling method with correlating mapping, i.e., the method of [116] to learn multiphysics data for two cases, case 1 being with fewer multiphysics training data (25 sets of data) and case 2 being with more multiphysics training data ( 81 sets of data). In this example, since the geometrical parameters $\left(h_{c 1}\right.$ and $\left.h_{c 2}\right)$ are influenced by (or correlated with) the non-geometrical parameters $\left(V_{1}\right.$ and $\left.V_{2}\right)$, the correlating mapping between the geometrical parameters $\left(h_{c 1}\right.$ and $\left.h_{c 2}\right)$ and the non-geometrical parameters $\left(V_{1}\right.$ and $\left.V_{2}\right)$ is established using the method of [116]. The multiphysics data are directly used for the training of Neuro-TF model with correlating mapping. 
Table 3.2 and Table 3.3 compare different parametric modeling methods in terms of ANN structures, average training and testing error, and CPU time. From the table we can see that when fewer multiphysics data is used, our proposed model is more accurate than the other two parametric models because our model has the knowledge of the coarse model trained with many inexpensive EM (single-physics) data. With the similar accuracy requirement, our proposed model uses fewer multiphysics data and less computation cost than the other two parametric models. The proposed multiphysics model provides accurate and fast prediction of multiphysics responses for high-level multiphysics design. Table 3.4 compares the computation time between the pure multiphysics non-parametric simulation (using COMSOL MULTIPHYSICS) and the proposed multiphysics parametric model w.r.t. different number of multiphysics simulations. From the table we can see that since the training is a one time investment, the benefit of using the proposed multiphysics model accumulates when the model is used over and over again with repetitive changes in physical/geometrical parameters.

The comparison of the magnitude in decibels of $\boldsymbol{S}_{11}$ of the proposed multiphysics model trained with less data (25 sets of data), the Neuro-TF model with correlating mapping trained with less data (25 sets of data), and the Neuro-TF model with correlating mapping trained with more data (81 sets of data) for two different filter geometries which are from testing data and have never been used in training process are shown in Fig. 3.10. The values of the input variables to our model for the two samples of the tunable cavity filter are as follows.

Test sample \#1: 
Table 3.2: Accuracy Comparisons of Different Methods for Parametric Modeling of the Four-Pole Waveguide Filter Example

\begin{tabular}{c|c|c|c|c}
\hline \hline Training Method & $\begin{array}{c}\text { No. of } \\
\text { EM Data }\end{array}$ & $\begin{array}{c}\text { No. of } \\
\text { Multiphysics } \\
\text { Data }\end{array}$ & $\begin{array}{c}\text { Average } \\
\text { Training } \\
\text { Error }\end{array}$ & $\begin{array}{c}\text { Average } \\
\text { Testing } \\
\text { Error }\end{array}$ \\
\hline $\begin{array}{c}\text { ANN Model Using Less } \\
\text { Multiphysics Training Data }\end{array}$ & 0 & 25 & $1.47 \%$ & $13.3 \%$ \\
\hline $\begin{array}{c}\text { ANN Model Using More } \\
\text { Multiphysics Training Data }\end{array}$ & 0 & 81 & $1.86 \%$ & $1.96 \%$ \\
\hline $\begin{array}{c}\text { Neuro-TF Model With } \\
\text { Correlating Mapping Using Less } \\
\text { Multiphysics Training Data }\end{array}$ & 0 & 25 & $1.56 \%$ & $11.6 \%$ \\
\hline $\begin{array}{c}\text { Neuro-TF Model With } \\
\text { Correlating Mapping Using More } \\
\text { Multiphysics Training Data }\end{array}$ & 0 & 81 & $1.53 \%$ & $1.67 \%$ \\
\hline $\begin{array}{c}\text { Proposed Model Using Less } \\
\text { Multiphysics Training Data }\end{array}$ & 81 & 25 & $1.56 \%$ & $1.63 \%$ \\
\hline \hline
\end{tabular}

$\boldsymbol{x}=\left[\begin{array}{llllll}3.49 & 4.23 & 3.3325 & 3.0775 & 25 & -175\end{array}\right]^{T}[\mathrm{~mm} \mathrm{~mm} \mathrm{~mm} \mathrm{~mm} \mathrm{~V} \mathrm{~V}]$

Test sample \#2:

$\boldsymbol{x}=\left[\begin{array}{llllll}3.59 & 4.25 & 3.2275 & 3.0325 & 125 & -125\end{array}\right]^{T}[\mathrm{~mm} \mathrm{~mm} \mathrm{~mm} \mathrm{~mm} \mathrm{~V} \mathrm{~V}]$

It is observed that compared to the simulation results performed with the COMSOL MULTIPHYSICS, our proposed multiphysics model can achieve good accuracy for different input samples even though these samples are never used in training. Once the overall model training is completed, we can implement the trained model 
Table 3.3: CPU Comparisons of Different Methods for Parametric Modeling of the Four-Pole Waveguide Filter Example

\begin{tabular}{|c|c|c|c|c|}
\hline Training Method & $\begin{array}{l}\text { Multiphysics } \\
\text { Data Gene } \\
\text {-ration Time }\end{array}$ & $\begin{array}{c}\text { EM Single } \\
\text { Physics Data } \\
\text { Generation Time }\end{array}$ & $\begin{array}{c}\text { Model } \\
\text { Training } \\
\text { Time }\end{array}$ & $\begin{array}{c}\text { Total } \\
\text { CPU Time }\end{array}$ \\
\hline $\begin{array}{c}\text { ANN Model Using } \\
\text { Less Multiphysics } \\
\text { Training Data }\end{array}$ & $12.1 \mathrm{~h}$ & 0 & $0.1 h$ & $12.2 h$ \\
\hline $\begin{array}{c}\text { ANN Model Using } \\
\text { More Multiphysics } \\
\text { Training Data }\end{array}$ & $39.8 h$ & 0 & $0.1 h$ & $39.9 h$ \\
\hline $\begin{array}{c}\text { Neuro-TF Model With } \\
\text { Correlating Mapping } \\
\text { Using Less Multiphysics } \\
\text { Training Data }\end{array}$ & $12.1 \mathrm{~h}$ & 0 & $0.2 h$ & $12.3 h$ \\
\hline $\begin{array}{l}\text { Neuro-TF Model With } \\
\text { Correlating Mapping } \\
\text { Using More Multiphysics } \\
\text { Training Data }\end{array}$ & $39.8 h$ & 0 & $0.2 h$ & $40 h$ \\
\hline $\begin{array}{c}\text { Proposed Model Using } \\
\text { Less Multiphysics } \\
\text { Training Data }\end{array}$ & $12.1 h$ & $2.4 h$ & $0.2 h$ & $14.7 h$ \\
\hline
\end{tabular}

into the design optimization where the design parameters can be repetitively adjusted during optimization. As an example of using the trained model with different values of geometrical and non-geometrical input parameters for the four-pole waveg- 
Table 3.4: Comparison of Computation Time Between Multiphysics Non-parametric Simulation and Proposed Multiphysics Parametric Model of the Four-Pole Waveguide Filter Example

\begin{tabular}{c|c|c}
\hline \hline \multirow{2}{*}{$\begin{array}{c}\text { No. of Changes } \\
\text { of Physical/Geome } \\
\text {-trical Parameters }\end{array}$} & $\begin{array}{c}\mid c \\
\text { CPU Time } \\
\text {-physics Model }\end{array}$ & $\begin{array}{c}\text { Simulation Using } \\
\text { Multiphysics Software }\end{array}$ \\
\cline { 2 - 3 } 1 & $\begin{array}{c}14.7 h \text { (model } \\
\text { development) }+0.008 s\end{array}$ & $0.51 h$ \\
\hline 100 & $\begin{array}{c}14.7 h \text { (model } \\
\text { development) }+0.8 s\end{array}$ & approx. $50 h$ \\
\hline 500 & $\begin{array}{c}14.7 h(\text { model } \\
\text { development) }+4 s\end{array}$ & approx. $250 h$ \\
\hline \hline
\end{tabular}

uide filter, we perform multiphysics optimization of two separate cavity filters with two different design specifications:

Specifications for cavity filter $\# 1$ : $\left|S_{11}\right| \leq-24 \mathrm{~dB}$ at

frequency range from $10.75 \mathrm{GHz}$ to $11.05 \mathrm{GHz}$.

Specifications for cavity filter $\# 2$ : $\left|S_{11}\right| \leq-25 \mathrm{~dB}$ at

frequency range from $10.85 \mathrm{GHz}$ to $11.15 \mathrm{GHz}$.

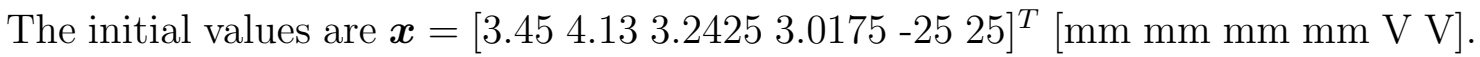
The design optimization using the proposed overall multiphysics model took only about 20 seconds to achieve the optimal design solution for each cavity filter. The optimized design parameter values for these two separate cavity filters are:

Filter \#1: 


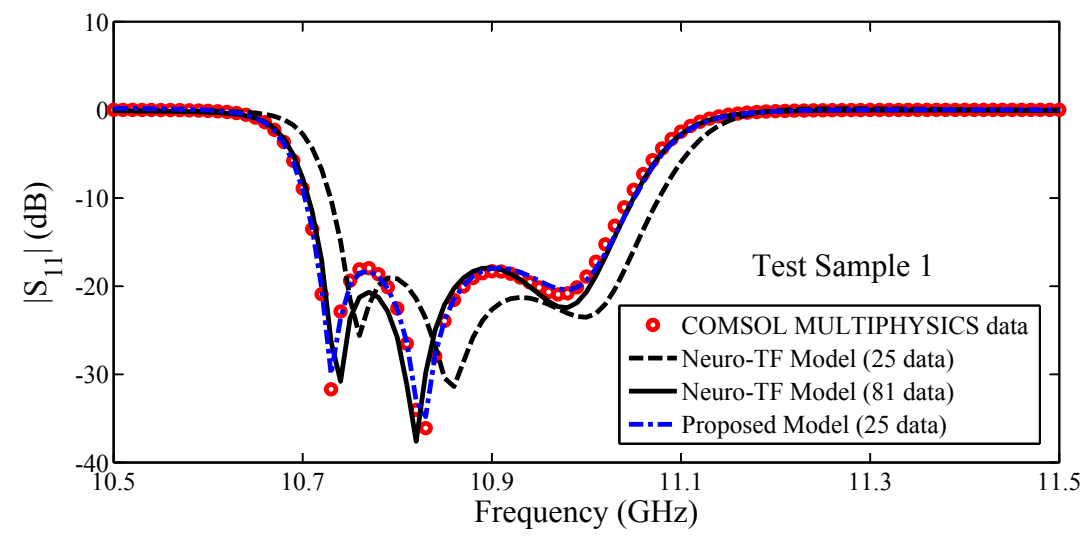

(a)

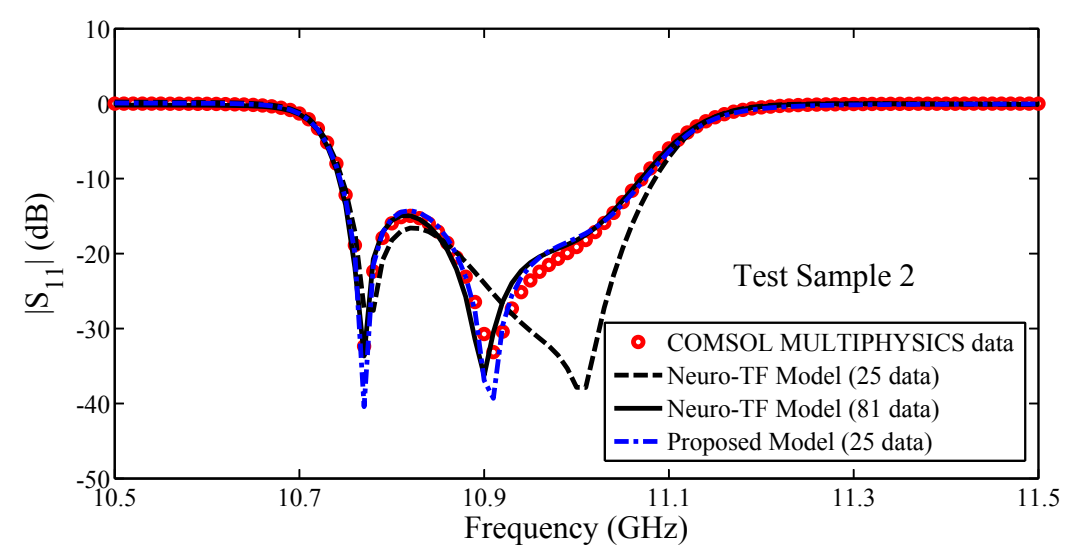

(b)

Figure 3.10: Comparison of the magnitude in decibels of $S_{11}$ of the overall multiphysics models developed using different modeling methods and COMSOL MULTIPHYSICS data: (a) Test sample \#1 and (b) Test sample \#2 for the four-pole waveguide example. In the figure, Neuro-TF model (less data) means the NeuroTF model with correlating mapping trained with less multiphysics data. Neuro-TF model (more data) means the Neuro-TF model with correlating mapping trained with more multiphysics data. Proposed model (less data) means the proposed model trained with less multiphysics data. 
$\boldsymbol{x}=\left[\begin{array}{llll}3.48373 & 4.17073 & 3.25362 & 2.98028 \\ 397.636 & 235.752\end{array}\right]^{T}[\mathrm{~mm} \mathrm{~mm} \mathrm{~mm} \mathrm{~mm} \mathrm{~V} \mathrm{~V}]$

Filter \#2:

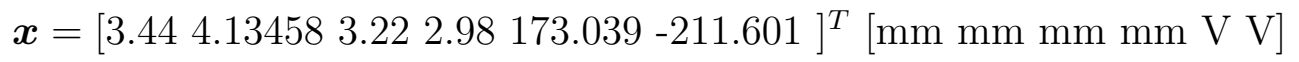

The magnitudes in decibels of $S_{11}$ and $S_{21}$ of COMSOL MULTIPHYSICS data at the model optimal solutions are shown in Fig. 3.11. Our multiphysics model can behave well in design optimization with different specifications. If we eliminate the effects of the other physics domains and consider only the EM single physics (EM only) simulation, in this example that means $V_{1}=0$ and $V_{2}=0$, we can get the EM response with other four geometrical parameters. We can still use our proposed model to do the optimization with only the four geometrical parameters. Fig. 3.12 (a) shows the optimization result with the specifications for the cavity filter $\left|S_{11}\right| \leq$ -25dB at frequency range from $10.80 \mathrm{GHz}$ to $11.10 \mathrm{GHz}$. The optimized geometrical

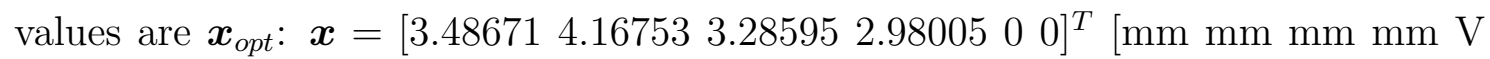
V]. In order to show the multiphysics effects and its tunability, we perform the optimization using only the two non-geometrical variables as the tuning variables, i.e., $V_{1}$ and $V_{2}$ while the other four geometrical variables are fixed during the tuning optimization process. With the same starting point as shown in Fig. 3.12 (a), we perform multiphysics optimization to determine values of tuning parameters to match the two different design specifications as in (17) and (18).

The initial point of the tuning optimization is $V_{1}=0 \mathrm{~V}$ and $V_{2}=0 \mathrm{~V}$ and the optimized tuning design parameter values for the two different specifications are:

Specification \#1: $V_{1}=230.429 \mathrm{~V}$ and $V_{2}=233.428 \mathrm{~V}$ 


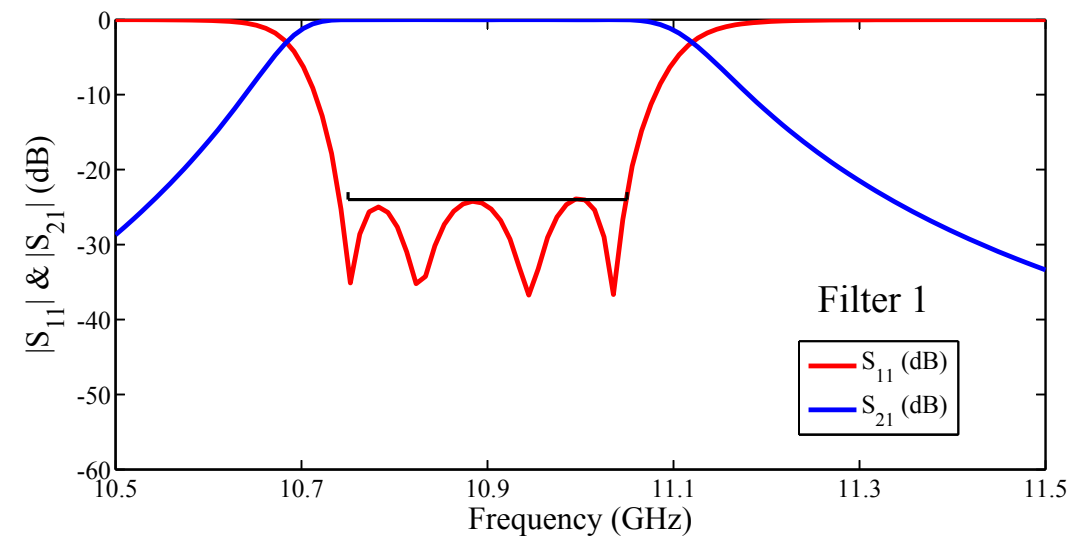

(a)

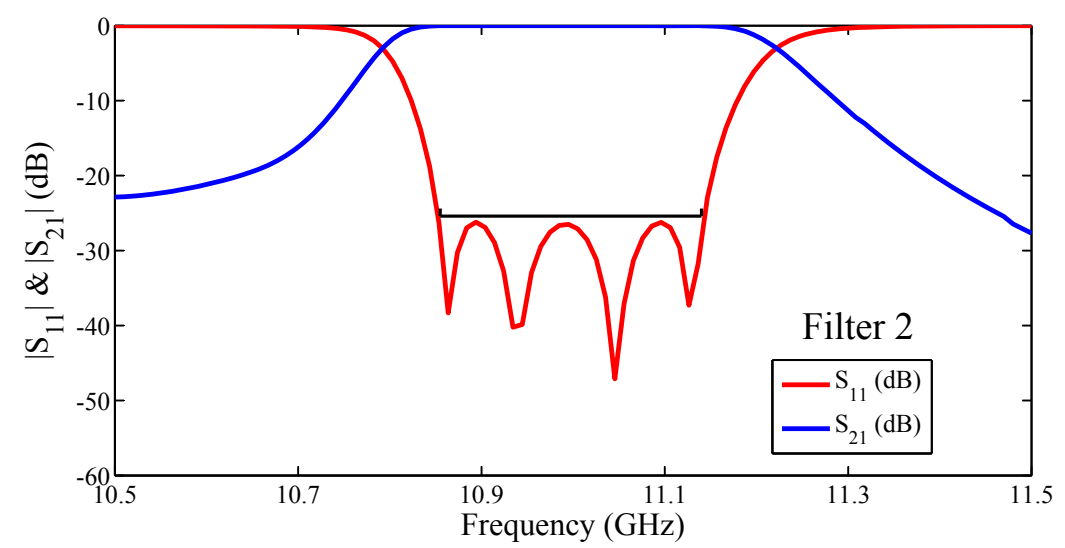

(b)

Figure 3.11: The proposed parametric model is used for optimization w.r.t. two separate cavity filters with two different specifications for the four-pole waveguide filter. The optimal solution is found by our model and verified by the COMSOL MULTIPHYSICS. The magnitude in decibels of $S_{11}$ and $S_{21}$ of COMSOL MULTIPHYSICS data at (a) Optimized design solution for filter 1 and (b) Optimized design solution for filter 2. As shown in the figure, the proposed model behaves well in design optimization with different specifications.

Specification \#2: $V_{1}=-244.46 \mathrm{~V}$ and $V_{2}=-236.589 \mathrm{~V}$

The COMSOL MULTIPHYSICS simulations at the optimal tuning solutions are 
shown in Fig. 3.12 (b) and (c). From the figure we can see that non-geometrical parameters are used for tuning the cavity filter to tune the cavity filter to get the desired response.

Our proposed multiphysics parametric model can provide similar results to those simulated with the commercial multiphysics tools within the training ranges as shown in Table II. If the multiphysics design parameters are slightly beyond the training ranges, our proposed model can still be used to get approximate results.

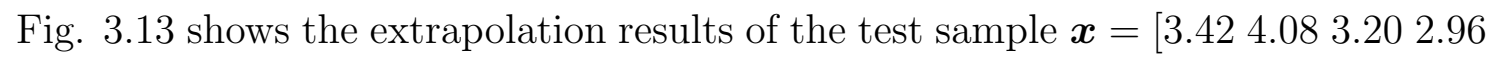
$-450-450]^{T}[\mathrm{~mm} \mathrm{~mm} \mathrm{~mm} \mathrm{~mm} \mathrm{~V} \mathrm{~V}]$. From the figure we can see that the results become approximate since the design parameters are slightly beyond the training ranges. If the multiphysics design parameters are far beyond the training ranges, our model cannot guarantee the reliability of the results.

Similarly, for the frequency extrapolation, if the frequency is slightly beyond the training frequency ranges, our proposed model can still be used to get approximate results. If the frequency is far beyond the training frequency ranges, our model cannot provide reliable results. Fig. 3.14 shows the frequency extrapolation results of the test sample $\boldsymbol{x}=\left[\begin{array}{llllll}3.49 & 4.23 & 3.3325 & 3.0775 & 25 & -175\end{array}\right]^{T}[\mathrm{~mm} \mathrm{~mm} \mathrm{~mm} \mathrm{~mm} \mathrm{~V} \mathrm{~V}]$ in the frequency range from $9.5 \mathrm{GHz}$ to $12 \mathrm{GHz}$. The training frequency range in this four-pole waveguide filter example are from $10.5 \mathrm{GHz}$ to $11.5 \mathrm{GHz}$. From the figure, we can see that our proposed model becomes less accurate in the frequency range from $9.5 \mathrm{GHz}$ to $10.1 \mathrm{GHz}$. 


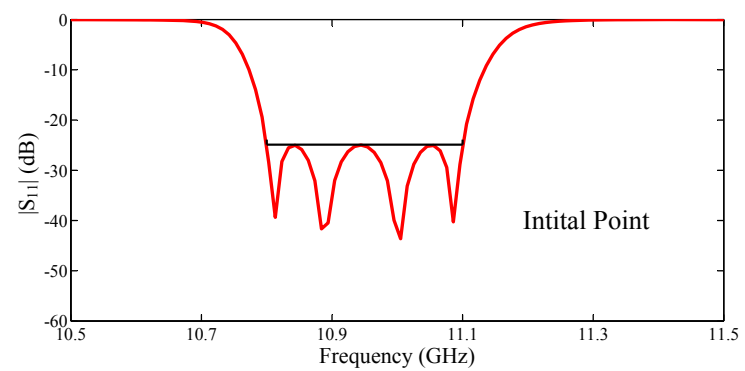

(a)

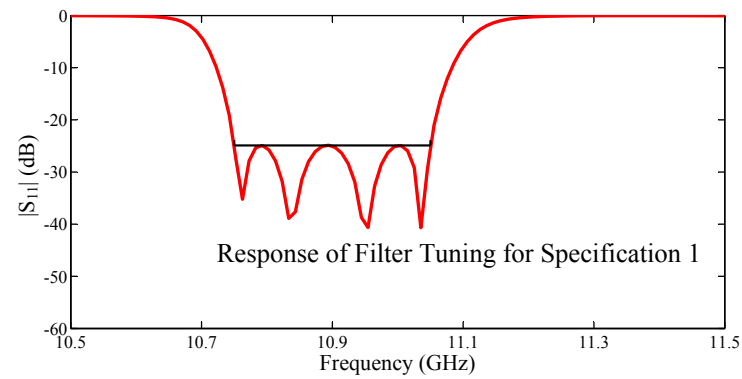

(b)

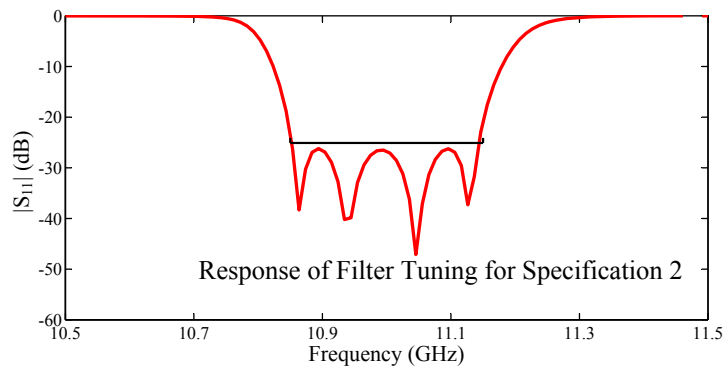

(c)

Figure 3.12: The proposed parametric model is used for filter tuning w.r.t. two different specifications for the four-pole waveguide filter. The optimal tuning solution is found by our model and verified by the COMSOL MULTIPHYSICS. The magnitude in decibels of $S_{11}$ of COMSOL MULTIPHYSICS data at (a) Initial point: $V_{1}=0 \mathrm{~V}$ and $V_{2}=0 \mathrm{~V}$, (b) Optimized tuning solution for specification 1: $V_{1}=230.429 \mathrm{~V}$ and $V_{2}=233.428 \mathrm{~V}$ and (c) Optimized tuning solution for specification $2: V_{1}=-244.4$ $\mathrm{V}$ and $V_{2}=-236.589 \mathrm{~V}$. As shown in the figure, the proposed model behaves well in tuning optimization with different specifications. 


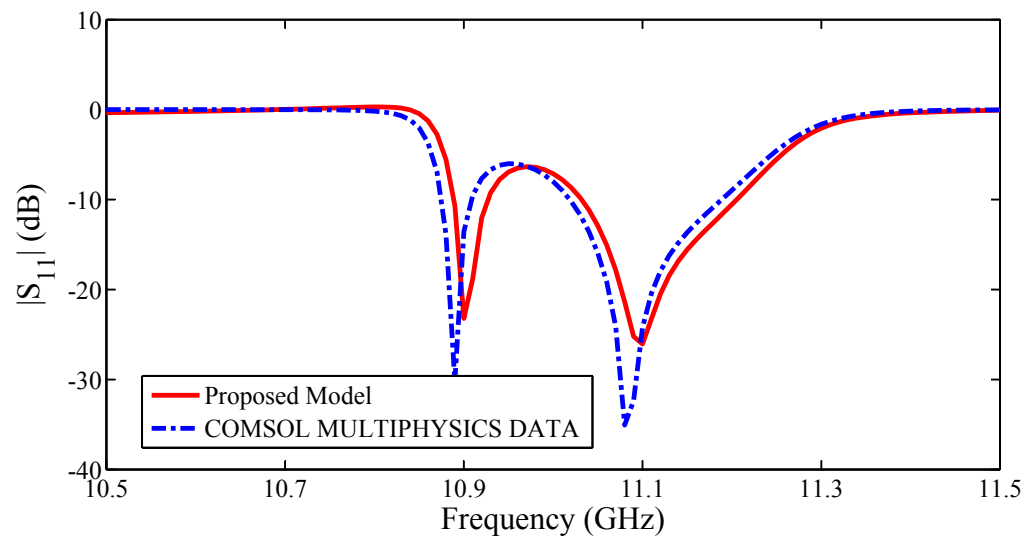

Figure 3.13: Extrapolation results of the test sample slightly beyond the training ranges for the four-pole waveguide filter example. We can see that the approximate results can be obtained by our proposed model.

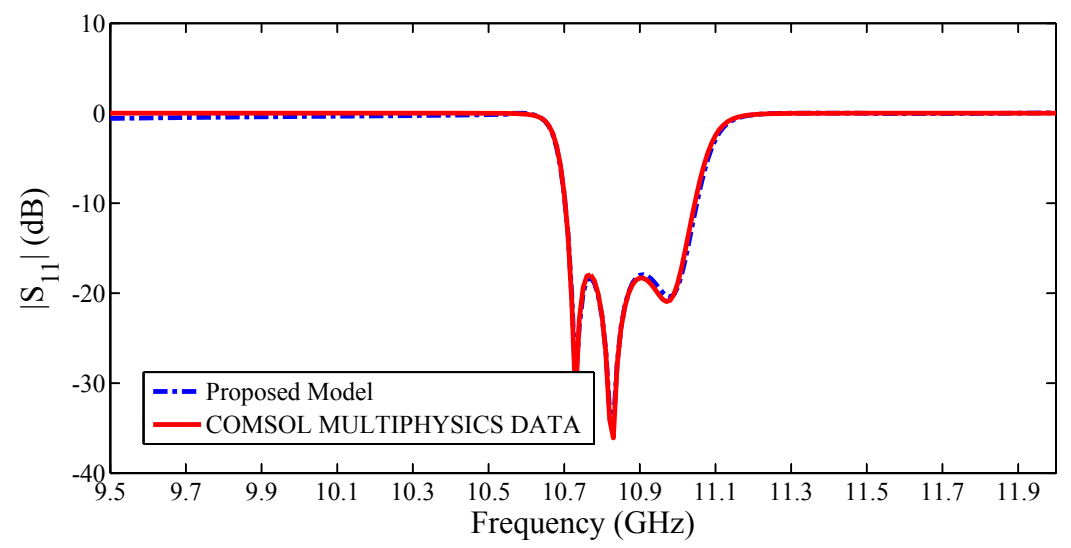

Figure 3.14: Frequency extrapolation results of the test sample in the frequency range from $9.5 \mathrm{GHz}$ to $12 \mathrm{GHz}$ for the four-pole waveguide filter example.

\subsubsection{Multiphysics Parametric Modeling of an Iris Coupled Microwave Cavity Filter}

In this example, we apply the proposed space mapped multiphysics model technique to an iris coupled cavity filter [127] shown in Fig. 3.15 (a). The filter has four 
geometrical design parameters, i.e., the iris widths $w_{1}, w_{2}, w_{3}$ and $w_{4}$. A large power $P_{i n}$ is supplied to the cavity filter as an additional design parameter which can change the EM single physics (EM only) responses due to the thermal effects and mechanical deformation as described in Section II. Frequency $f$ is an additional input. For this multiphysics problem, the design parameter includes five variables $\boldsymbol{x}=\left[\begin{array}{lllll}w_{1} & w_{2} & w_{3} & w_{4} & P_{i n}\end{array}\right]^{T}$. The geometrical inputs of the overall multiphysics model are $\boldsymbol{p}=\left[\begin{array}{llll}w_{1} & w_{2} & w_{3} & w_{4}\end{array}\right]^{T}$. the non-geometrical input variable is $\boldsymbol{q}=P_{i n}$. The model has two outputs, i.e., $\boldsymbol{y}=\left[\begin{array}{lll}R S_{11} & I S_{11}\end{array}\right]^{T}$, which are the real and imaginary parts of the overall multiphysics model output $S_{11}$ with different values of geometrical and nongeometrical parameters as variables. For the EM single physics (EM only) domain coarse model, we construct the coarse model which has the four input parameters $\boldsymbol{p}_{c}=\left[\begin{array}{llll}w_{1} & w_{2} & w_{3} & w_{4}\end{array}\right]^{T}$. Frequency $f_{c}$ is an additional input of the EM domain coarse model.

ANSYS WORKBENCH 17.1 including HFSS, Steady-State Thermal and Static Structural is used to perform the multiphysics simulation to generate the over all multiphysics model training and testing data w.r.t. different geometrical and nongeometrical design parameters. The actual process of this multiphysics problem is shown in Fig. 3.16. Three physics domains (EM, thermal and structural mechanics) are considered in this example and different domains are coupled affecting each other. The actual fine model using the entire mesh information to calculate the multiphysics responses while our technique uses the mapping functions to represent the output response changes caused by other physics domains. After the multiphysics simulation, the temperature information and structural deformation information of 


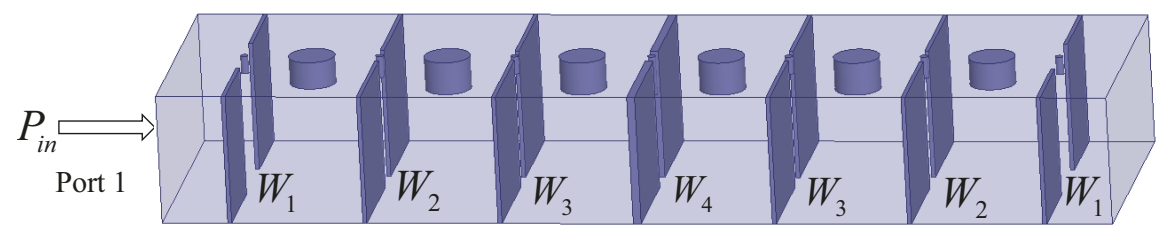

(a)

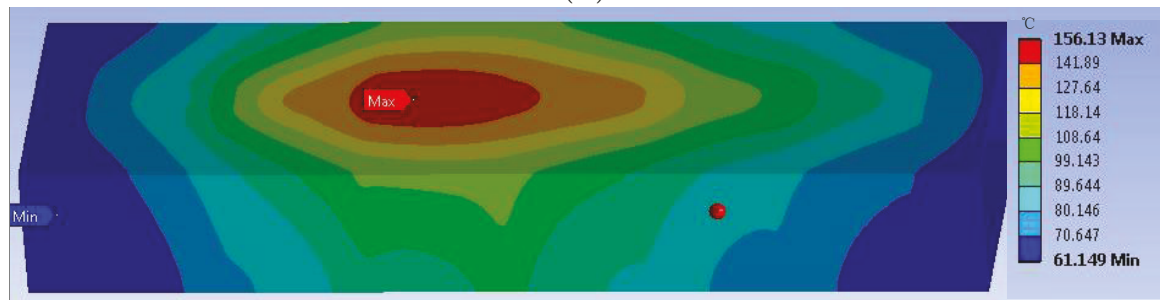

(b)

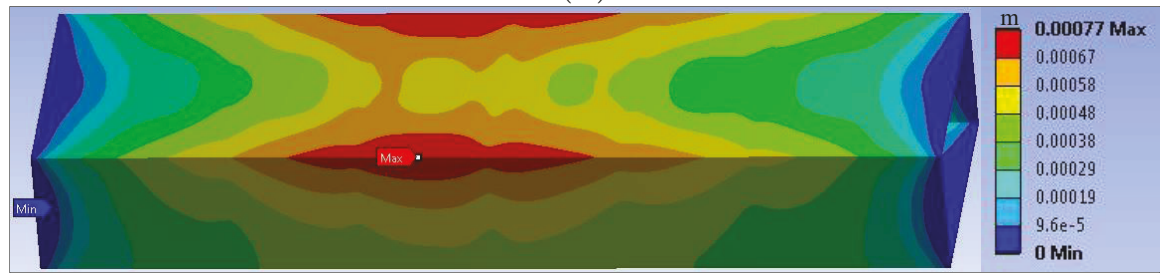

(c)

Figure 3.15: (a) Structure of the iris coupled waveguide filter where a high input power is supplied to port 1. The design variables are $\boldsymbol{x}=\left[\begin{array}{lllll}w_{1} & w_{2} & w_{3} & w_{4} & P_{\text {in }}\end{array}\right]^{T}$, (b) Temperature distribution in the iris coupled waveguide filter caused by the large input power and (c) Structural deformation in the iris coupled waveguide filter caused by the temperature distribution.

the cavity filter with the design parameter $\boldsymbol{x}=\left[\begin{array}{llll}116.5 & 49.735 & 43.445 & 48.995 \\ 36.25\end{array}\right]^{T}$ [mm mm mm mm kW] are shown in Fig. 3.15 (b) and Fig. 3.15 (c) respectively. We can see that due to the large input power, the power loss generates the heat in the cavity filter and causes the deformation of the filter structure. These deformations make the outputs of the multiphysics simulation different from the EM single physics (EM only) simulation. Fig. 3.17 shows the output responses using EM single physics (EM only) simulation and multiphysics simulation for this cavity 


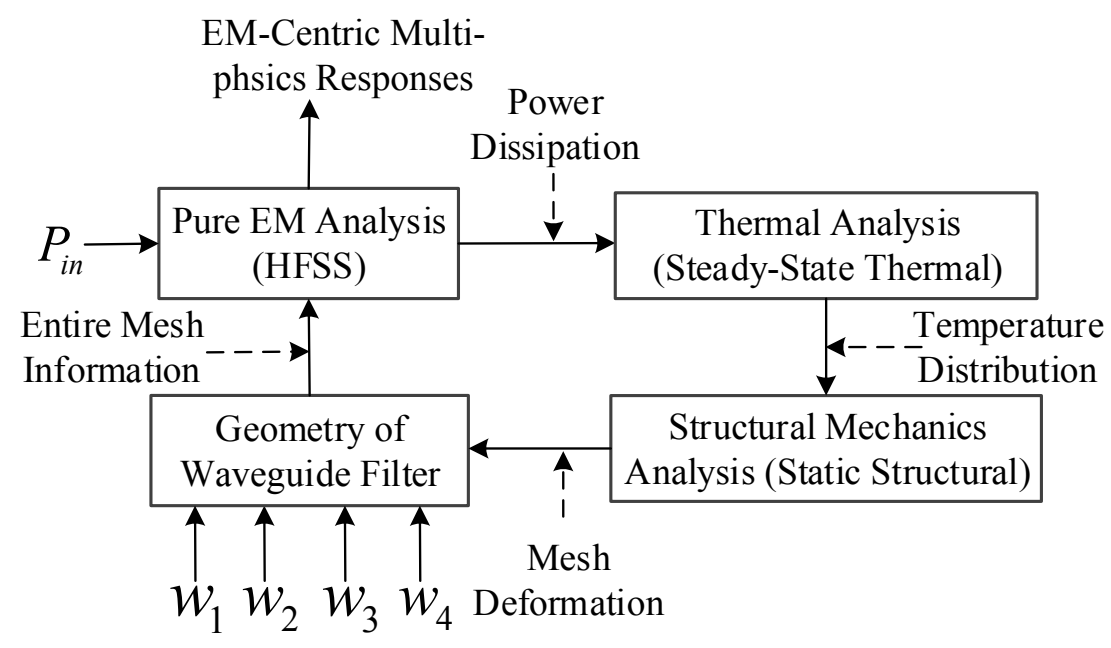

Figure 3.16: The actual process of the multiphysics simulation for the iris coupled waveguide filter example using the ANSYS WORKBENCH software.

filter example, i.e., the EM domain coarse model response and overall multiphysics overall model response using the same geometrical parameters. From the figure we can see the single physics analysis is not accurate enough to represent the multiphysics responses. Our multiphysics model is more accurate because we include other physics domain besides the EM domain effects into our model.

For EM domain coarse model data generation w.r.t. different geometrical parameters as variables, the EM single physics (EM only) evaluation is performed by ANSYS HFSS EM simulator using the fast simulation feature. DOE method is used as the sampling method for both EM domain coarse and overall multiphysics model data generation.

The EM single physics (EM only) simulation data used to construct the EM domain coarse model with geometrical parameters as variables uses nine levels of DOE for defining samples of the training data, i.e., a total of 81 samples of training 


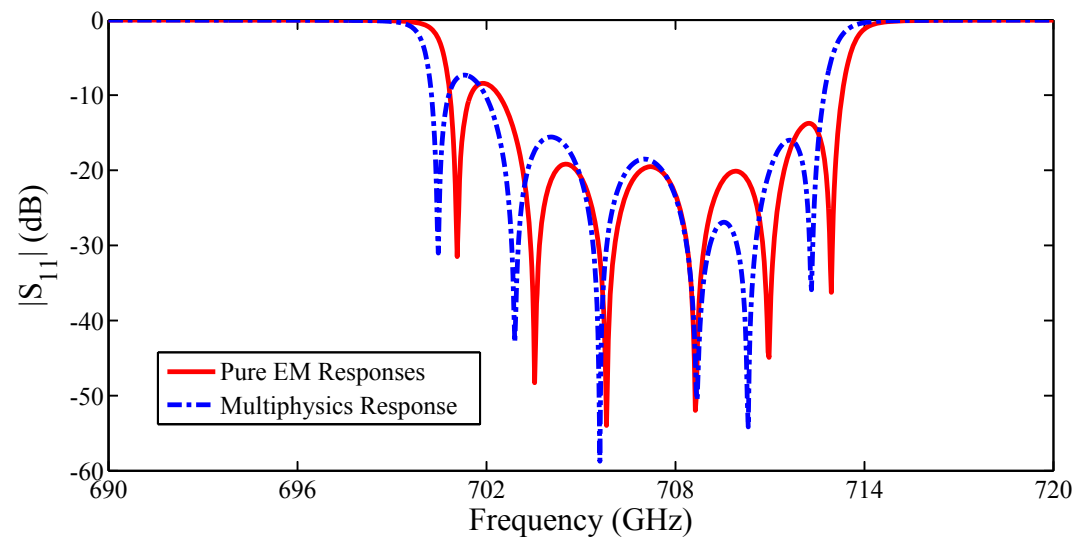

Figure 3.17: Comparison of the magnitude in decibels of $S_{11}$ of the EM single physics (EM only) response and multiphysics analysis response using the same geometrical parameters for the iris coupled waveguide filter. From the figure we can see that without mapping, the single physics analysis is not accurate enough to represent the multiphysics responses.

data, and eight levels of DOE for defining samples of the testing data, i.e., a total of 64 samples of testing data. While for the overall multiphysics model data, we only use five levels of DOE for defining samples of the multiphysics training data, i.e., a total of 25 samples of training data. The input ranges of the geometrical variables for the EM domain coarse model should be larger than the overall multiphysics model to accommodate the mapping between the EM domain and multiphysics domain. The physical shape of the training and testing structure for this example is shown in Fig. 3.15 (a) and the specific values of training data and testing data for both coarse model and overall model are shown in Table 3.5. The testing data are randomly selected within the training ranges and never used in the training process. The frequency range for model development is from $690 \mathrm{MHz}$ to $720 \mathrm{MHz}$.

For this example, the three-layer perception neural network is used to construct 
Table 3.5: Definition of Training and Testing Data for EM Domain Coarse Model and Overall Multiphysics Fine Model For the Iris Coupled Waveguide Filter

\begin{tabular}{|c|c|c|c|c|c|c|c|}
\hline \multirow{2}{*}{\multicolumn{2}{|c|}{$\begin{array}{l}\text { Input Variables } \\
\text { to the Model }\end{array}$}} & \multicolumn{3}{|c|}{ Training Data Range } & \multicolumn{3}{|c|}{ Testing Data Range } \\
\hline & & Min & Max & Step & Min & Max & Step \\
\hline \multirow{4}{*}{$\begin{array}{l}\text { EM Data } \\
\text { (Coarse } \\
\text { Model) }\end{array}$} & $\begin{array}{c}w_{1} \\
(\mathrm{~mm})\end{array}$ & 111.93 & 118.73 & 0.85 & 112.355 & 118.305 & 0.85 \\
\hline & $\begin{array}{c}w_{2} \\
(\mathrm{~mm})\end{array}$ & 48.66 & 51.86 & 0.4 & 48.86 & 51.66 & 0.4 \\
\hline & $\begin{array}{c}w_{3} \\
(\mathrm{~mm})\end{array}$ & 43.13 & 45.93 & 0.35 & 43.305 & 45.755 & 0.35 \\
\hline & $\begin{array}{c}w_{4} \\
(\mathrm{~mm})\end{array}$ & 46.65 & 49.69 & 0.38 & 46.84 & 49.5 & 0.38 \\
\hline \multirow{5}{*}{$\begin{array}{c}\text { Multi- } \\
\text { physics } \\
\text { Data } \\
\text { (Fine } \\
\text { Mdodel) }\end{array}$} & $\begin{array}{c}w_{1} \\
(\mathrm{~mm})\end{array}$ & 112.21 & 118.45 & 1.56 & 112.6 & 118.06 & 0.78 \\
\hline & $\begin{array}{c}w_{2} \\
(\mathrm{~mm})\end{array}$ & 48.86 & 51.26 & 0.7 & 49.035 & 51.485 & 0.35 \\
\hline & $\begin{array}{c}w_{3} \\
(\mathrm{~mm})\end{array}$ & 43.29 & 45.77 & 0.62 & 43.445 & 45.615 & 0.31 \\
\hline & $\begin{array}{c}w_{4} \\
(\mathrm{~mm})\end{array}$ & 46.85 & 49.49 & 0.66 & 47.015 & 49.325 & 0.33 \\
\hline & $\begin{array}{c}P_{i n} \\
(\mathrm{~kW})\end{array}$ & 20 & 40 & 5 & 21.25 & 38.75 & 2.5 \\
\hline
\end{tabular}

the EM domain coarse model with geometrical parameters as variables. The number of hidden neurons of the neural networks that represent the relationships between the geometrical parameters and EM single physics (EM only) responses is 40 . The coarse model is trained using the NeuroModelerPlus software. The average training error for the EM domain (single physics) coarse model development is 1.65\%, while 
the average testing error is $1.58 \%$. After an accurate EM domain coarse model is developed, we can continue to set up the overall multiphysics model which can accurately represent the multiphysics data. The overall multiphysics model including ANN coarse model and two mapping neural networks is also constructed and trained using the NeuroModelerPlus software. Numbers of hidden neurons for the two mapping neural network modules are 6 and 2 respectively. The average training error for the overall multiphysics model development is $1.83 \%$, while the average testing error is $1.92 \%$. The overall multiphysics model training process takes about 8 minutes including EM domain (single physics) coarse model and overall multiphysics model developments.

In this example, the correlating information between the non-geometrical parameter $P_{\text {in }}$ and geometrical parameters $\boldsymbol{p}$ is not available. Therefore the method in [116] is not applicable. Our proposed technique can work well even when the correlating information needed in[116] is not available. We perform the parametric modeling using the direct method without correlating mapping for comparison purpose. ANN model (i.e., without mapping) is directly trained to learn multiphysics data for two cases, case 1 being with fewer multiphysics training data (25 sets of data) and case 2 being with more multiphysics training data (81 sets of data). Table 3.6 and Table 3.7 compare different parametric modeling methods in terms of ANN structures, average training and testing error, and CPU time. From the table we can see that when fewer multiphysics data is used, our proposed model is more accurate than the direct method because our model has the knowledge of the coarse model trained with many inexpensive EM (single-physics) data. With the 
similar accuracy requirement, our proposed model uses fewer multiphysics data and less computation cost than direct multiphysics modeling methods. The proposed multiphysics model provides accurate and fast prediction of multiphysics responses for high-level design. Table 3.8 compares the computation time between the pure multiphysics non-parametric simulation (using ANSYS WORKBENCH) and the proposed multiphysics parametric model w.r.t. different number of testing samples. From the table we can see that since the training is a one time investment, the benefit of using the proposed multiphysics model accumulates when the model is used over and over again with repetitive changes in physical/geometrical parameters.

The comparison of the magnitude in decibels of $\boldsymbol{S}_{11}$ of the proposed model trained with less data (25 sets of data), direct ANN model trained with less data (25 sets of data), and direct ANN model trained with more data (81 sets of data) for two different filter geometries which are from testing data and have never been used in training process are shown in Fig. 3.18. The values of the input variables to our model for two samples of the high power cavity filter are as follows.

Test sample \#1:

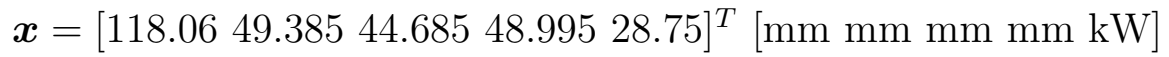

Test sample \#2:

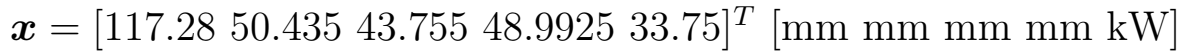

It is observed that compared to the simulation results performed with the ANSYS WORKBENCH, our proposed multiphysics model can achieve good accuracy for different input samples even though these samples are never used in training. 
Table 3.6: Accuracy Comparisons of Different Methods for Parametric Modeling of the Iris Coupled Waveguide Filter

\begin{tabular}{c|c|c|c|c}
\hline \hline Training Method & $\begin{array}{c}\text { No. of } \\
\text { EM Data }\end{array}$ & $\begin{array}{c}\text { No. of } \\
\text { Multiphysics } \\
\text { Data }\end{array}$ & $\begin{array}{c}\text { Average } \\
\text { Training } \\
\text { Error }\end{array}$ & $\begin{array}{c}\text { Average } \\
\text { Testing } \\
\text { Error }\end{array}$ \\
\hline $\begin{array}{c}\text { ANN Model Using Less } \\
\text { Multiphysics Training Data }\end{array}$ & 0 & 25 & $1.86 \%$ & $9.56 \%$ \\
\hline $\begin{array}{c}\text { ANN Model Using More } \\
\text { Multiphysics Training Data }\end{array}$ & 0 & 81 & $1.78 \%$ & $1.86 \%$ \\
\hline $\begin{array}{c}\text { Proposed Model Using Less } \\
\text { Multiphysics Training data }\end{array}$ & 81 & 25 & $1.83 \%$ & $1.92 \%$ \\
\hline \hline
\end{tabular}

Once the overall model training is completed, we can implement the trained multiphysics model into the design optimization where the design parameters can be repetitively adjusted during optimization. As an example of using the trained model for the iris waveguide filter, we perform multiphysics optimization of two separate cavity filters using two different starting points:

Initial values for cavity filter \#1: $\boldsymbol{x}=\left[\begin{array}{lllll}113.21 & 48.96 & 43.35 & 46.85 & 25.25\end{array}\right]^{T}[\mathrm{~mm}$ $\mathrm{mm} \mathrm{mm} \mathrm{mm} \mathrm{kW}]$.

Initial values for cavity filter \#2: $\boldsymbol{x}=\left[\begin{array}{l}116.45 \\ 50.66\end{array} 45.9749 .6535 .5\right]^{T}[\mathrm{~mm} \mathrm{~mm}$ $\mathrm{mm} \mathrm{mm} \mathrm{kW}]$.

The specification for these two filters is $\left|S_{11}\right| \leq-20 \mathrm{~dB}$ at frequency range from $702 \mathrm{MHz}$ to $712 \mathrm{MHz}$. The design optimization using the proposed overall multi- 
Table 3.7: CPU Comparisons of Different Methods for Parametric Modeling of the Iris Coupled Waveguide Filter

\begin{tabular}{c|c|c|c|c}
\hline \hline Training Method & $\begin{array}{c}\text { Multiphysics } \\
\text { Data Gene } \\
\text {-ration Time }\end{array}$ & $\begin{array}{c}\text { EM Single } \\
\text { Physics Data } \\
\text { Generation Time }\end{array}$ & $\begin{array}{c}\text { Model } \\
\text { Training } \\
\text { Time }\end{array}$ & $\begin{array}{c}\text { Total } \\
\text { CPU Time }\end{array}$ \\
\hline $\begin{array}{c}\text { ANN Model Using } \\
\text { Less Multiphysics } \\
\text { Training Data }\end{array}$ & $50 h$ & 0 & $0.1 h$ & $50.1 h$ \\
\hline $\begin{array}{c}\text { ANN Model Using } \\
\text { More Multiphysics } \\
\text { Training Data }\end{array}$ & $162 h$ & 0 & $0.1 h$ & $162.1 h$ \\
\hline $\begin{array}{c}\text { Proposed Model Using } \\
\text { Less Multiphysics } \\
\text { Training data }\end{array}$ & $50 h$ & $28.8 h$ & $0.2 h$ & $79 h$ \\
\hline \hline
\end{tabular}

physics model took only about 20 seconds to achieve the optimal design solution for each cavity filter. The optimized design parameter values for these two separate cavity filters are:

Filter \#1:

$\boldsymbol{x}=\left[\begin{array}{ll}115.699 & 50.915 \\ 45.0664 & 48.604821 .25\end{array}\right]^{T}[\mathrm{~mm} \mathrm{~mm} \mathrm{~mm} \mathrm{~mm} \mathrm{kW].}$

Filter \#2:

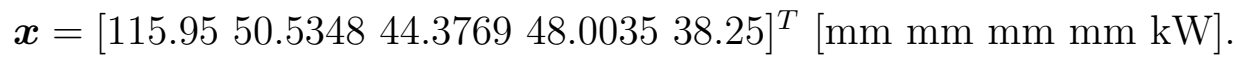

The ANSYS WORKBENCH performs the multiphysics simulations at the model 
Table 3.8: Comparison of Computation Time Between Multiphysics Non-parametric Simulation and Proposed Multiphysics Parametric Model of the Iris Coupled Waveguide Filter

\begin{tabular}{c|c|c}
\hline \hline \multirow{2}{*}{$\begin{array}{c}\text { No. of Changes } \\
\text { of Physical/Geome } \\
\text {-trical Parameters }\end{array}$} & $\begin{array}{c}\mid c \\
\text { CPU Time } \\
\text {-physics Model }\end{array}$ & $\begin{array}{c}\text { Simulation Using } \\
\text { Multiphysics Software }\end{array}$ \\
\cline { 2 - 3 } 1 & $\begin{array}{c}79 h \text { (model } \\
\text { development) }+0.008 s\end{array}$ & $2.03 h$ \\
\hline 100 & $\begin{array}{c}79 h \text { (model } \\
\text { development) }+0.8 s\end{array}$ & approx. 200 h \\
\hline 500 & $\begin{array}{c}79 h \text { (model } \\
\text { development) }+4 s\end{array}$ & approx. 1000 h \\
\hline \hline
\end{tabular}

optimal solutions and the multiphysics responses meet the required specifications. Our proposed EM centric multiphysics model can behave well in design optimization.

\subsection{Conclusion}

In this chapter, a space mapped multiphysics parametric modeling technique has been proposed to develop an efficient multiphysics parametric model for microwave components. In the proposed method, we use the EM single physics (EM only) behaviors w.r.t different values of geometrical parameters in non-deformed structure of microwave components as the coarse model. Two mapping module functions have been formulated to map the EM domain responses to the multiphysics domain 


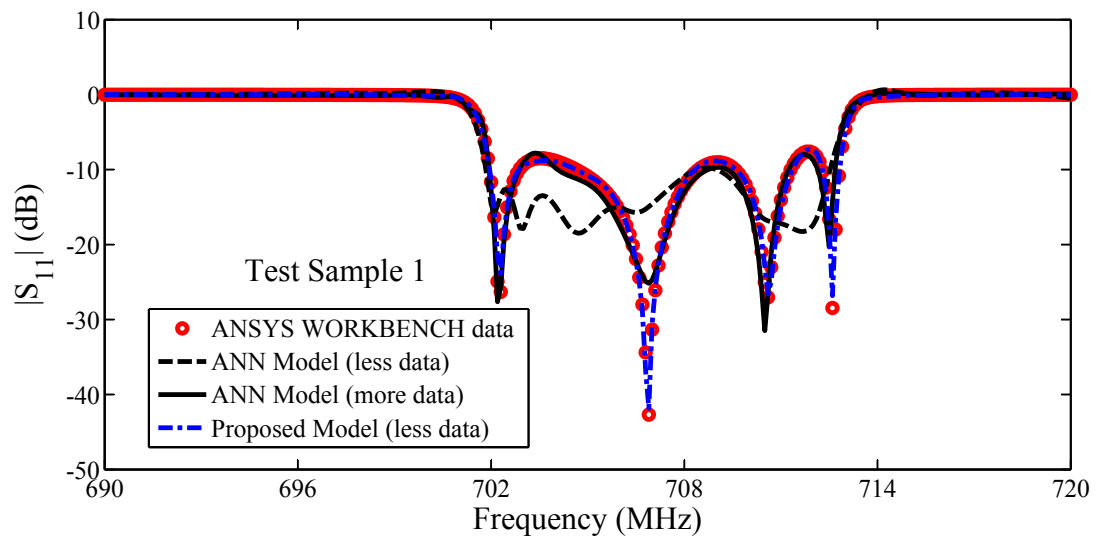

(a)

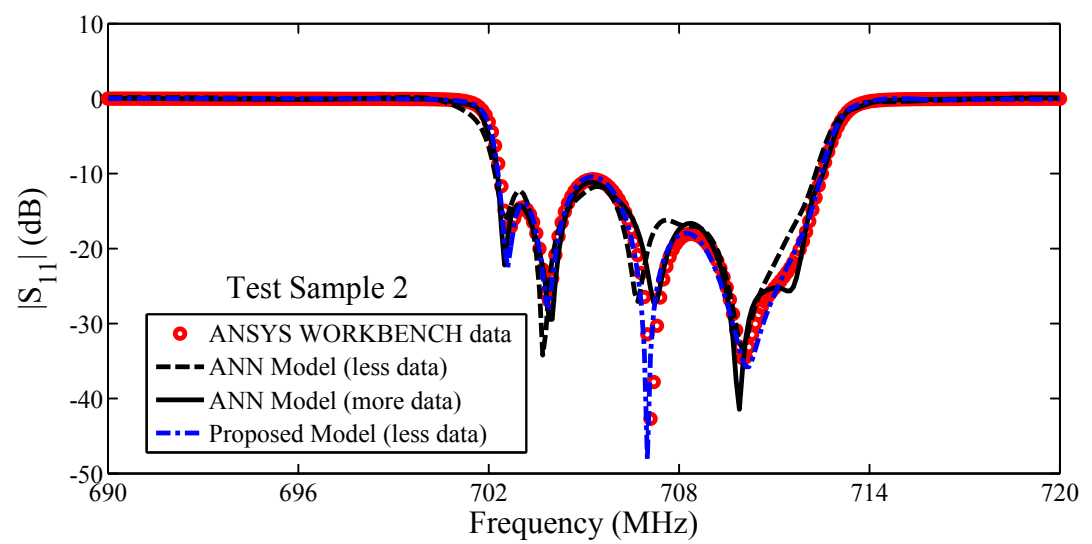

(b)

Figure 3.18: Comparison of the magnitude in decibels of $S_{11}$ of the models developed using different modeling methods and ANSYS WORKBENCH data: (a) Test sample \#1 and (b) Test sample \#2 for the iris waveguide filter. In the figure, ANN model (less data) means the ANN model trained with less multiphysics data. ANN model (more data) means the ANN model trained with more multiphysics data. Proposed model (less data) means the proposed model trained with less multiphysics data.

responses. Our proposed technique can achieve good accuracy of the multiphysics model with fewer multiphysics training data and less computational cost than di- 
rect multiphysics parametric modeling. After the proposed multiphysics modeling process, the trained multiphysics model can be used to provide accurate and fast prediction of multiphysics analysis responses of microwave components with geometrical and non-geometrical design parameters as variables. The developed overall model can be also used for high-level EM centric multiphysics design and optimization. We have used two microwave waveguide filter examples to illustrate our proposed method in the chapter. The proposed technique for multiphysics parametric model can be applied to other passive microwave component modeling with physical parameters as variables. 


\section{Chapter 4}

\section{EM Centric Multiphysics Optimization of Microwave Components Using Parallel Computational Approach}

In this chapter, for the first time, a novel parallel EM centric multiphysics optimization technique is developed. In our proposed technique, the pole-residue-based transfer function is exploited to build an effective and robust surrogate model [42]. A group of modified quadratic mapping functions is formulated to map the relationships between pole/residues of the transfer function and the design variables. Multiple EM centric multiphysics evaluations are performed in parallel to generate the training samples for establishing the surrogate model. Using our proposed technique, the surrogate model can be valid in a relatively large neighborhood which makes an effective and large optimization update in each optimization iteration. The trust region algorithm is performed to guarantee the convergence of the proposed multiphysics optimization algorithm. Our proposed multiphysics optimiza- 
tion technique takes a small number of iterations to obtain the optimal EM centric multiphysics response.

\subsection{Introduction}

Design optimization of microwave components often requires a large number of repetitive simulations to obtain the optimum design space parameters. For electromagnetic (EM) design, directly using EM simulation to perform the design optimization is computationally very expensive considering that EM-simulation-driven optimization requires repetitive EM simulations to adjust the values of design variables $[5]-[8]$.

Considering a more challenging situation, for the high-performance microwave component and system design, besides the EM physics domain, we usually need to consider the operation in the real world multi-physics environment which contains the effects of other physics domains. Multiphysics analysis typically encompasses multiple physics domain analysis, such as EM, structural mechanics and thermal. This makes the multiphysics simulation even more computationally expensive and time-consuming in comparison to the single physics pure EM simulations [34]. We considers an even more challenging scenario over [34] for design optimization, i.e., EM centric multi-physics optimization. Design optimization of EM centric multiphysics behaviors of the microwave components is even more time-consuming since it requires repetitively multiphysics evaluations due to the adjustments of the values of design variables. Developing efficient multiphysics optimization methods becomes a challenging task to speed up the multiphysics design process. 
In this chapter, for the first time, a novel parallel EM centric multiphysics optimization technique is developed to accelerate the multiphysics design process. In the proposed technique, multiple EM centric multiphysics evaluations are generated simultaneously using parallel computation for developing the surrogate model in each optimization iteration. The pole-residue-based transfer function [86] is exploited to build an effective and robust surrogate model to represent the EM-centric multiphysics responses. A group of modified quadratic mapping functions is formulated to map the relationships between pole/residues of the transfer function and the design parameters. The surrogate model is valid in a relatively large neighborhood which makes a large and effective optimization update in each optimization iteration. The trust region algorithm is performed to allow the trust radius to change dynamically iteration by iteration which guarantees the convergence of the proposed multiphysics optimization algorithm. Therefore, the proposed optimization takes a small number of optimization iterations to obtain the optimal EM centric multiphysics response.

\subsection{Parallel EM Centric Multiphysics Optimiza- tion}

Let $\boldsymbol{x}$ represent a vector containing all the design variables of a given $\mathrm{RF} /$ microwave device, defined as

$$
\boldsymbol{x}=\left[\begin{array}{llll}
x_{1} & x_{2} & \cdots & x_{N}
\end{array}\right]^{T}
$$

where $N$ is the total number of multiphysics design variables for the parametric model. For establishing an efficient surrogate model for multiphysics optimization, 
we first divide the design variables into two sets, which are geometrical variables and multiphysics variables. Let $\boldsymbol{x}_{m}$ be defined as the vector containing all the multiphysics variables in the vector $\boldsymbol{x} . \boldsymbol{x}_{m}$ represents all the non-geometrical variables in other multiphysics domains besides geometrical variables. The multiphysics design variables can influence the EM responses typically by indirectly affecting the geometrical variables. Thereby, we further divide geometrical variables into two sets, which are geometrical variables $\boldsymbol{x}_{g m}$ that can be much affected by multiphysics variables and the other geometrical variables $\boldsymbol{x}_{g}$ which can be least or not affected by multiphysics variables. Therefore, the vector of design variables can be defined as,

$$
\boldsymbol{x}=\left[\begin{array}{lll}
\boldsymbol{x}_{g}^{T} & \boldsymbol{x}_{g m}^{T} & \boldsymbol{x}_{m}^{T}
\end{array}\right]^{T}
$$

The design variables are divided into three sets in (4.2), which are $\boldsymbol{x}_{g}, \boldsymbol{x}_{g m}$, and $\boldsymbol{x}_{m}$. Let $n_{1}, n_{2}$, and $n_{3}$ represent the number of variables in $\boldsymbol{x}_{g}, \boldsymbol{x}_{g m}$, and $\boldsymbol{x}_{m}$, respectively. $n$ represents the total number of variables in $\boldsymbol{x}$, i.e., $N=n_{1}+n_{2}+n_{3}$.

\subsubsection{Parallel EM Centric Multiphysics Evaluation}

In the proposed method, the first step for the surrogate modeling process is the generation of EM centric multiphysics training samples. There are various distribution methods for generating the data, such as grid distribution, star distribution, and orthogonal distribution. In the proposed technique, orthogonal distribution [125], i.e., a specific type of design of experiment (DOE) sampling distribution, is used for generating multiple sample points where the subspace divisions are sam- 


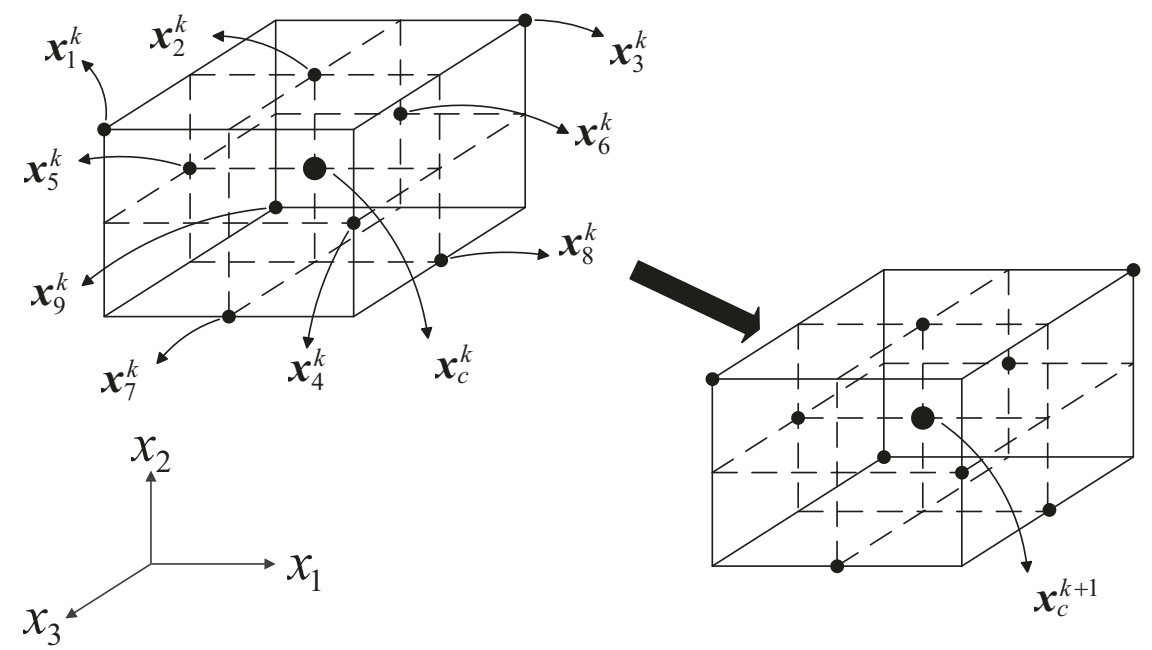

Figure 4.1: Illustration of multiple samples used to train the surrogate model. The orthogonal distribution is used to generate the multiple samples $\left(\widehat{\boldsymbol{x}}_{1}^{k}, \widehat{\boldsymbol{x}}_{2}^{k}, \cdots, \widehat{\boldsymbol{x}}_{n_{s}}^{k}\right)$ around the central point $\boldsymbol{x}_{c}^{k}$. The central point $\boldsymbol{x}_{c}^{k}$ is updated after each iteration of the proposed optimization method. The updated central point $\boldsymbol{x}_{c}^{k+1}$ and the orthogonal samples around the new central point move as the proposed optimization progresses from the $k$ th iteration to the $(k+1)$ th iteration.

pled with the same density and are orthogonal. Orthogonal distribution around the central point uses far fewer sampling points in comparison to grid distribution and enables the surrogate model to be valid in much larger neighborhood in comparison to star distribution. Let $k$ represent the index of optimization iterations which is initialized to one. The index $k$ of optimization iteration increases by one after each optimization iteration. Fig. 4.1 shows the orthogonal distribution for generating the multiple samples around a central point $\boldsymbol{x}_{c}^{k}$ for the $k$ th iteration. Let $\widehat{\boldsymbol{x}}_{j}^{k}$ denote one such sample, where $j \in\left\{1,2, \cdots, n_{s}\right\}$ and $n_{s}$ represents the total number of samples using orthogonal distribution. When the optimization process moves, the central point $\boldsymbol{x}_{c}^{k}$ moves to a new optimization update (new central point) $\boldsymbol{x}_{c}^{k+1}$. All 
the other orthogonal sample points $\left(\widehat{\boldsymbol{x}}_{1}^{k}, \widehat{\boldsymbol{x}}_{2}^{k}, \cdots, \widehat{\boldsymbol{x}}_{n_{s}}^{k}\right)$ move along with the central point when the optimization progresses from the $k$ th iteration to the $(k+1)$ th iteration.

In the proposed technique, multiple EM centric multiphysics evaluations for constructing the surrogate model take the major computational burden of the total computational time. Sequential EM centric multiphysics evaluations of the samples requires $n_{s}$ times the computational time of one EM centric multiphysics evaluation. Therefore, to reduce the overall computational time, we propose to use parallel computational approach for the EM centric multiphysics evaluations. EM centric multiphysics responses $\widehat{y}_{m}\left(\widehat{\boldsymbol{x}}_{j}^{k}, s\right)$ are generated for the set of samples, $j=1,2, \cdots, n_{s}$, simultaneously by simulating multiple multiphysics structures in parallel,

$$
\begin{aligned}
\left\{\widehat{y}_{m}\left(\widehat{\boldsymbol{x}}_{j}^{k}, s\right) \mid j=1,2, \cdots, n_{s}\right\} & \\
& =\left\{\widehat{y}_{m}\left(\widehat{\boldsymbol{x}}_{1}^{k}, s\right), \widehat{y}_{m}\left(\widehat{\boldsymbol{x}}_{2}^{k}, s\right), \cdots, \widehat{y}_{m}\left(\widehat{\boldsymbol{x}}_{n_{s}}^{k}, s\right)\right\}
\end{aligned}
$$

After the parallel data generation, the generated multiple EM centric multiphysics data can be used to develop the surrogate model.

\subsubsection{Proposed Surrogate Model for EM Centric Multi- physics Optimization}

The surrogate model response is expressed as a transfer function in the pole-residue format [86]. The proposed surrogate model is shown in Fig. 4.2. The model consists of pole-residue-based transfer functions and a group of modified quadratic mapping functions. The outputs of the overall model are the $S$-parameters of the EM centric 


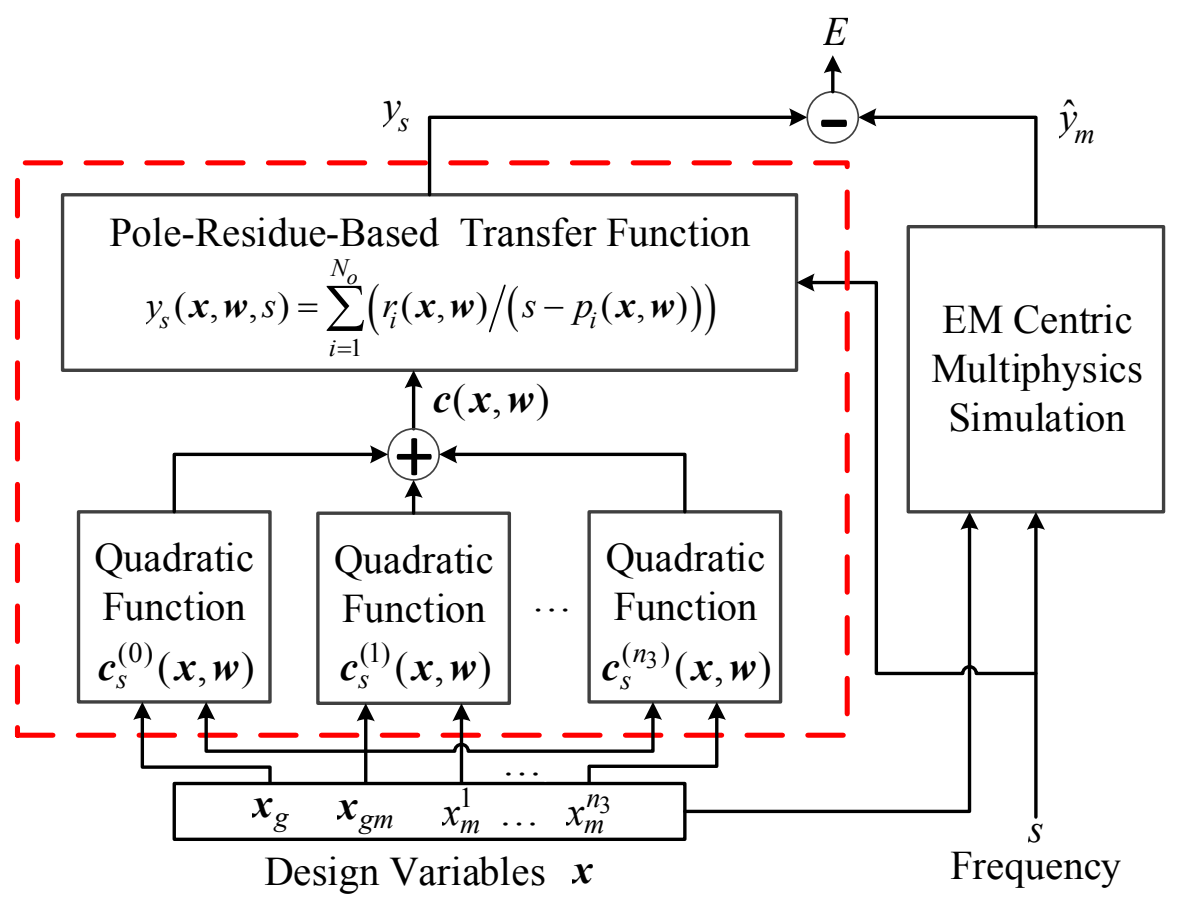

Figure 4.2: The structure of the proposed surrogate model with pole/residue based transfer function. The proposed model consists of a transfer function and $n_{3}+1$ quadratic functions, where $\boldsymbol{w}$ represents the weighting parameters in the quadratic functions; $\boldsymbol{x}$ represents the design parameters; $y_{s}$ represents the surrogate model outputs; $\widehat{y}_{m}$ represents the data from EM centric multiphysics simulation.

multiphysics behaviors of microwave components and the inputs of the model are design variables and frequency.

Let $s$ represent complex angular frequency. Let the frequency response $y_{s}(\boldsymbol{x}, s)$ be the surrogate model output, which is defined using a pole-residue-based transfer function as follows,

$$
y_{s}(\boldsymbol{x}, s)=\sum_{i=1}^{N_{o}} \frac{r_{i}(\boldsymbol{x}, s)}{s-p_{i}(\boldsymbol{x}, s)}
$$

where $p_{i}$ and $r_{i}$ represent the poles and residues of the transfer function respectively, 
and $N_{o}$ represents the order of the transfer function. Let $\boldsymbol{c}$ be a vector including all the poles and residues of the transfer function, defined as

$$
\begin{aligned}
\boldsymbol{c} & =\left[c_{i}\right]^{T} \\
& =\left[\begin{array}{llllllll}
p_{1} & p_{2} & \cdots & p_{N_{o}} & r_{1} & r_{2} & \cdots & r_{N_{o}}
\end{array}\right]^{T}
\end{aligned}
$$

where $c_{i}$ is the $i$ th element in $\boldsymbol{c}$, where $i=1,2, \ldots, 2 N_{o}$.

We propose to use a modified quadratic mapping function to represent the relationship between the pole/residues of the transfer function and the input variables. Let $c_{i}$ represent the output response of the modified quadratic mapping function. Let $\boldsymbol{w}_{i}$ represent the vector containing all the weighting parameters in the quadratic function $c_{i}$. The modified quadratic mapping function is formulated as a sum of multiple sub quadratic functions $c_{s}^{i,(l)}$ representing different relationships. The first sub quadratic function $c_{s}^{i,(0)}$ represents the pole/residues with respect to the geometrical variables, formulated as,

$$
\begin{aligned}
& c_{s}^{i,(0)}\left(\boldsymbol{x}, \boldsymbol{w}_{i}\right)=w_{i}^{0}+w_{i}^{1} x_{g}^{1}+w_{i}^{2} x_{g}^{2}+\cdots+w_{i}^{n_{1}} x_{g}^{n_{1}} \\
& \quad+w_{i}^{\left(n_{1}+1\right)} x_{g m}^{1}+w_{i}^{\left(n_{1}+1\right)} x_{g m}^{2}+\cdots+w_{i}^{\left(n_{1}+n_{2}\right)} x_{g m}^{n_{2}} \\
& \quad+w_{i}^{\left(n_{1}+n_{2}+1\right)}\left(x_{g}^{1}\right)^{2}+w_{i}^{\left(n_{1}+n_{2}+2\right)} x_{g}^{1} x_{g}^{2}+\cdots \\
& \quad+w_{i}^{\left(2 n_{1}+2 n_{2}\right)} x_{g}^{1} x_{g m}^{n_{2}}+\cdots+w_{i}^{n_{t}}\left(x_{g m}^{n_{2}}\right)^{2}
\end{aligned}
$$

where

$$
n_{t}=\frac{\left(n_{1}+n_{2}\right)\left(n_{1}+n_{2}+3\right)}{2}
$$


There is a correlation between the multiphysics variables $\boldsymbol{x}_{m}$ and the multiphysics affected geometrical variables $\boldsymbol{x}_{g m}$. The remaining sub quadratic functions $c_{s}^{i,(l)}$ represent the combined effects of the multiphysics variables $\boldsymbol{x}_{m}$ and the multiphysics affected geometrical variables $\boldsymbol{x}_{g m}$ on the pole/residues, formulated as,

$$
\begin{aligned}
c_{s}^{i,(l)}\left(\boldsymbol{x}, \boldsymbol{w}_{i}\right)= & w_{i}^{\left(n_{t}+(l-1)\left(n_{2}+2\right)+1\right)} x_{m}^{l} \\
& +w_{i}^{\left(n_{t}+(l-1) *\left(n_{2}+2\right)+2\right)} x_{g m}^{1} x_{m}^{l}+\cdots \\
& +w_{i}^{\left(n_{t}+(l-1)\left(n_{2}+2\right)+3\right)} x_{g m}^{n_{2}} x_{m}^{l} \\
& +w_{i}^{\left(n_{t}+l\left(n_{2}+2\right)\right)}\left(x_{m}^{l}\right)^{2}
\end{aligned}
$$

where $l$ represents the index of the element in the multiphysics variables $\boldsymbol{x}_{m}$, i.e., $l=$ $1,2, \ldots, n_{3}$. Therefore, there are $n_{3}+1$ sub quadratic functions for constructing the proposed modified quadratic function. The proposed modified quadratic function is derived as the sum of all the $n_{3}+1$ quadratic functions, formulated as,

$$
c_{i}\left(\boldsymbol{x}, \boldsymbol{w}_{i}\right)=\sum_{l=0}^{n_{3}} c_{s}^{i,(l)}\left(\boldsymbol{x}, \boldsymbol{w}_{i}\right)
$$

where $\boldsymbol{w}_{i}$ represent the vector containing all the weighting parameters in the quadratic function $c_{i}$, formulated as,

$$
\boldsymbol{w}_{i}=\left[\begin{array}{llll}
w_{i}^{0} & w_{i}^{1} \cdots & w^{\left(n_{w}-1\right)}
\end{array}\right]^{T}
$$

where $n_{w}$ represents the total number of weighting parameters, formulated as,

$$
n_{w}=\frac{\left(n_{1}+n_{2}\right)\left(n_{1}+n_{2}+3\right)}{2}+n_{3}\left(n_{2}+2\right)+1
$$

The number of unknowns in (4.10) is dependent on the size of design variables $\boldsymbol{x}$ 
in the EM centric multiphysics optimization problem. The number of multiphysics samples selected using orthogonal distribution must be greater than the number of unknown weighting parameters for a good modeling accuracy.

The modified quadratic mapping function is formulated to map the relationship between pole/residues of the transfer function and the design variables. To obtain the modified quadratic mapping function, the data of poles and residues w.r.t. the design variables for all the training samples in each optimization iteration need to be obtained. The vector fitting process [87] is performed to obtain a set of poles and residues for each training sample. In the vector fitting process, the given information is EM centric multiphysics data $\widehat{y}_{m}\left(\widehat{\boldsymbol{x}}_{j}^{k}\right)$ (i.e., $S$-parameters) versus frequency for a training sample. Expected solutions are poles and residues of the transfer function with order equal to $N_{o}$.

Let $\widehat{\boldsymbol{c}}_{j}^{k}$ represent a vector containing the data of poles and residues of the transfer function of the $j^{\text {th }}$ training sample in the $k$ th optimization iteration obtained after vector fitting, defined as

$$
\widehat{\boldsymbol{c}}_{j}^{k}=\left[\widehat{c}_{j, i}^{k}\right]^{T}=\left[\widehat{p}_{j, 1}^{k} \widehat{p}_{j, 2}^{k} \cdots \widehat{p}_{j, N_{o o}}^{k} \widehat{r}_{j, 1}^{k} \widehat{r}_{j, 2}^{k} \cdots \widehat{r}_{j, N_{o o}}^{k}\right]^{T}
$$

where $\widehat{c}_{j, i}^{k}$ represents the $i$ th element in $\widehat{\boldsymbol{c}}_{j}^{k}$, where $i=1,2, \ldots, 2 N_{o}$.

Let $\widehat{x}_{j, l}^{k}$ represent the value of the $l$ th design variable of the $j$ th training sample in the $k$ th optimization iteration. The formulation for solving $\boldsymbol{w}_{i}$ for the $i$ th quadratic function is derived in (4.13) where $\beta=n_{1}+n_{2}$. 


$$
\left[\begin{array}{c}
\widehat{c}_{1, i}^{k} \\
\widehat{c}_{2, i}^{k} \\
\vdots \\
\widehat{c}_{j, i}^{k} \\
\vdots \\
\widehat{c}_{n_{s}, i}^{k}
\end{array}\right]=\left[\begin{array}{cccccccccc}
1 & \widehat{x}_{1,1}^{k} & \cdots & \widehat{x}_{1, \beta}^{k} & \left(\widehat{x}_{1,1}^{k}\right)^{2} & \cdots & \left(\widehat{x}_{1, \beta}^{k}\right)^{2} & \widehat{x}_{1, \beta+1}^{k} & \cdots & \left(\widehat{x}_{1, n}^{k}\right)^{2} \\
1 & \widehat{x}_{2,1}^{k} & \cdots & \widehat{x}_{2, \beta}^{k} & \left(\widehat{x}_{2,1}^{k}\right)^{2} & \cdots & \left(\widehat{x}_{2, \beta}^{k}\right)^{2} & \widehat{x}_{2, \beta+1}^{k} & \cdots & \left(\widehat{x}_{2, n}^{k}\right)^{2} \\
\vdots & \vdots & \ddots & \vdots & \vdots & \ddots & \vdots & \vdots & \ddots & \vdots \\
1 & \widehat{x}_{j, 1}^{k} & \cdots & \widehat{x}_{j, \beta}^{k} & \left(\widehat{x}_{j, 1}^{k}\right)^{2} & \cdots & \left(\widehat{x}_{j, \beta}^{k}\right)^{2} & \widehat{x}_{j, \beta+1}^{k} & \cdots & \left(\widehat{x}_{j, n}^{k}\right)^{2} \\
\vdots & \vdots & \ddots & \vdots & \vdots & \ddots & \vdots & \vdots & \ddots & \vdots \\
1 & \widehat{x}_{n_{s}, 1}^{k} & \cdots & \widehat{x}_{n_{s}, \beta}^{k} & \left(\widehat{x}_{n_{s}, 1}^{k}\right)^{2} & \cdots & \left(\widehat{x}_{n_{s}, \beta}^{k}\right)^{2} & \widehat{x}_{n_{s}, \beta+1}^{k} & \cdots & \left(\widehat{x}_{n_{s}, n}^{k}\right)^{2}
\end{array}\right]\left[\begin{array}{c}
w_{i}^{0} \\
w_{i}^{1} \\
\vdots \\
w_{i}^{\beta} \\
w_{i}^{\beta+1} \\
\vdots \\
w_{i}^{n_{t}} \\
w_{i}^{\left(n_{t}+1\right)} \\
\vdots \\
w_{i}^{\left(n_{w}-1\right)}
\end{array}\right]
$$

Using traditional quadratic function to map all the design variables to the pole/residues, the total number of weighting parameters is $N(N+1) / 2+N+1$. By using the proposed modified quadratic mapping function for EM centric multiphysics optimization, the number of weighting parameters for each quadratic mapping function $c_{i}$, say the $i$ th quadratic mapping function, is reduced by $n_{d}$, where

$$
n_{d}=\frac{n_{3}\left(n_{3}-1\right)}{2}+n_{1} n_{3}
$$

Furthermore, the number $n_{s}$ of training samples required in (4.13) needs to be equal to or greater than the number $n_{w}$ of unknown weighting parameters for a good modeling accuracy. Using the proposed modified quadratic function, the minimum number of training samples, i.e., the time-consuming EM centric multiphysics evaluations, can be also reduced. The reduced number of EM centric multiphysics evaluations will result in less computer resources required for parallel computation 
and less overhead of parallel computation, consequently speeding up the overall EM centric multiphysics optimization.

\subsubsection{Proposed Parallel EM Centric Multiphysics Optimiza- tion}

In this subsection, we give a detailed explanation for how to use the proposed surrogate model to perform the EM centric multiphysics optimization. Firstly, we define $\boldsymbol{X}_{s}^{k}$ to represent the trained region of geometrical parameters of the surrogate model in the $k$ th iteration using the trust region algorithm,

$$
\begin{aligned}
\boldsymbol{X}_{s}^{k}=\left\{\boldsymbol{x} \mid x_{c, l}^{k}-\delta_{l}^{k} \leq\right. & x_{l} \leq x_{c, l}^{k}+\delta_{l}^{k} \\
& \text { where } l=1,2, \cdots, N\}
\end{aligned}
$$

where $x_{c, l}^{k}$ represents each design variable in the central point $\boldsymbol{x}_{c}^{k}$ in the $k$ th iteration, $l=1,2, \cdots, N . \delta_{l}^{k}$ determines the range of each design variable in the $k$ th iteration. $\boldsymbol{\delta}^{k}$ determines a vector consisting of the ranges $\delta_{l}^{k}$ of all the design variables in the $k$ th iteration, i.e., $\boldsymbol{\delta}^{k}=\left[\delta_{1}^{k} \delta_{2}^{k} \cdots \delta_{N}^{k}\right]$. The surrogate model is developed within the region of $\boldsymbol{X}_{s}^{k}$. The EM centric multiphysics response $\widehat{y}_{m}\left(\boldsymbol{x}_{c}^{k}, s\right)$ is evaluated at the central point $\boldsymbol{x}_{c}^{k}$ and multiple EM centric multiphysics data $\widehat{y}_{m}\left(\widehat{\boldsymbol{x}}_{j}^{k}, s\right), j=$

$1,2, \ldots, n_{s}$, following orthogonal distribution around $\boldsymbol{x}_{c}^{k}$ is generated simultaneously by simulating multiple multiphysics structures in parallel using (4.3).

After we obtain the training data, we establish the proposed surrogate model in the following steps. Firstly, we perform the parameter extraction by vector fitting and pole-residue tracking technique [86]. The second step is preliminary training for the quadratic functions in the surrogate model. The quadratic mapping 
functions of $\boldsymbol{c}_{s}^{(0)} \cdots \boldsymbol{c}_{s}^{\left(n_{3}\right)}$ are formulated using (4.6) - (4.9). The coefficients for the quadratic functions are determined by the vector-fitting data where the inputs are the multiphysics design parameters and the outputs are the pole/residues extracted from vector fitting using (4.12) - (4.13). The last step is the model refinement training process, where the overall surrogate model is further trained by the EM centric multiphysics data for all the training data. An optimization formulation for the refinement training of the surrogate model is used to minimize the sum of the squared differences between the EM centric multiphysics responses and the surrogate model responses at all the $n_{s}$ training samples, defined as

$$
\begin{gathered}
E(\boldsymbol{w})=\sum_{j=1}^{n_{s}} \sum_{m=1}^{N_{f}}\left\|y_{s}\left(\widehat{\boldsymbol{x}}_{j}^{k}, \boldsymbol{w}, s_{m}\right)-\widehat{y}_{m}\left(\widehat{\boldsymbol{x}}_{j}^{k}, s_{m}\right)\right\|^{2}, \\
\boldsymbol{w}^{k}=\arg \min _{\boldsymbol{w}} E(\boldsymbol{w}),
\end{gathered}
$$

where $\boldsymbol{w}^{k}$ contains the optimal weighting parameters of the surrogate model in the $k$ th optimization iteration. $N_{f}$ represents the number of frequencies. $E$ represents the sum of the squared differences between the EM centric multiphysics responses and the surrogate model responses at all the training samples.

Once the proposed surrogate model is established, we perform the design optimization using the surrogate model, formulated as,

$$
\boldsymbol{x}^{k}=\arg \min _{\boldsymbol{x} \in \boldsymbol{X}_{s}^{k}} U\left(y_{s}\left(\boldsymbol{x}, \boldsymbol{w}^{*}, s\right)\right),
$$

where $\boldsymbol{x}^{k}$ represents the optimal surrogate model solution after the $k$ th iteration.

Trust region framework is used to improve the convergence of the proposed 
EM centric multiphysics surrogate optimization. Let $U\left(\widehat{y}_{m}\left(\boldsymbol{x}^{k-1}\right)\right)$ and $U\left(\widehat{y}_{m}\left(\boldsymbol{x}^{k}\right)\right)$ represent the objective function responses calculated using EM centric multiphysics simulation at $\boldsymbol{x}^{k-1}$ and $\boldsymbol{x}^{k}$, respectively. Let $U\left(y_{s}\left(\boldsymbol{x}^{k-1}\right)\right)$ and $U\left(y_{s}\left(\boldsymbol{x}^{k}\right)\right)$ represent the objective function responses calculated using the surrogate model at $\boldsymbol{x}^{k-1}$ and $\boldsymbol{x}^{k}$, respectively. An adjustment control index parameter $r_{s}$ is calculated to represent the ratio of the actual reduction and the predicted reduction in the value of the proposed objective functions. The formulation of $r_{s}$ is derived as,

$$
r_{s}=\frac{U\left(y_{s}\left(\boldsymbol{x}^{k-1}\right)\right)-U\left(y_{s}\left(\boldsymbol{x}^{k}\right)\right)}{U\left(\widehat{y}_{m}\left(\boldsymbol{x}^{k-1}\right)\right)-U\left(\widehat{y}_{m}\left(\boldsymbol{x}^{k}\right)\right)} .
$$

The trust radius $\boldsymbol{\delta}^{k}$ is then updated based on the control index parameter $r_{s}$, calculated as,

$$
\boldsymbol{\delta}^{k+1}= \begin{cases}\eta_{e} \boldsymbol{\delta}^{k}, & r_{s}>0.85, \\ \boldsymbol{\delta}^{k}, & 0.4 \leq r_{s} \leq 0.85, \\ \eta_{c} \boldsymbol{\delta}^{k}, & r_{s}<0.4\end{cases}
$$

where $\eta_{e}$ and $\eta_{c}$ represent the coefficients for the trust radius update. Expansion and contraction of the trust radius depends on the values of $\eta_{e}$ and $\eta_{c}$ in (4.20), respectively. In our case, we use $\eta_{e}=1.2$ and $\eta_{c}=0.6$.

EM centric multiphysics data generation, surrogate model development, surrogate design optimization, and trust region update are performed iteratively. The proposed algorithm terminates if the normalized absolute difference between the design variables of the current iteration and the previous iteration is sufficiently small or if the EM centric multiphysics response reaches the design specifications, 
i.e.,

$$
\begin{gathered}
\left\|\frac{\boldsymbol{x}^{k}-\boldsymbol{x}^{k-1}}{\boldsymbol{x}^{k}}\right\| \leq \epsilon, \\
U\left(\widehat{y}_{m}\left(\boldsymbol{x}^{k}\right)\right) \leq 0,
\end{gathered}
$$

where $\epsilon$ is a user defined threshold (e.g., 10 $0^{-4}$ ). The computation process (4.3) and (4.12)-(4.22) is considered one surrogate optimization iteration. Once the termination condition (4.21) or (4.22) is either satisfied, the solution to the proposed optimization is obtained, and the optimization terminates in the $k$ th optimization iteration. The corresponding $\boldsymbol{x}^{k}$ represents the final optimal solutions with multiphysics simulation accuracy. Otherwise, if (4.21) and (4.22) are not satisfied, we set the central point for the next iteration equal to the optimal surrogate solution in the present iteration, i.e., $\boldsymbol{x}_{c}^{k+1}=\boldsymbol{x}^{k}$. We then increase the index of iteration by one, i.e., $k=k+1$, and begin the next surrogate optimization iteration (4.3) and (4.12)-(4.22). Parallel techniques are used in the proposed technique to efficiently establish the surrogate model. By using the efficient surrogate model to drive EM centric multiphysics optimization, the proposed technique can obtain a significant speedup over direct multiphysics optimization. The flowchart of the proposed EM centric multiphysics optimization technique using surrogate model is illustrated in Fig. 4.3. The proposed optimization algorithm is summarized as follows.

Step 1) Divide the design variables into three subsets $\boldsymbol{x}=\left[\boldsymbol{x}_{g}^{T} \boldsymbol{x}_{g m}^{T} \boldsymbol{x}_{m}^{T}\right]^{T}$. Set the starting point $\boldsymbol{x}^{0}$. Initialize $k=1$. Set initial central point $\boldsymbol{x}_{c}^{k}=\boldsymbol{x}^{0}$. Initialize the trust radius $\boldsymbol{\delta}^{k}$.

Step 2) Use orthogonal distribution sampling strategy to generate a set of samples 


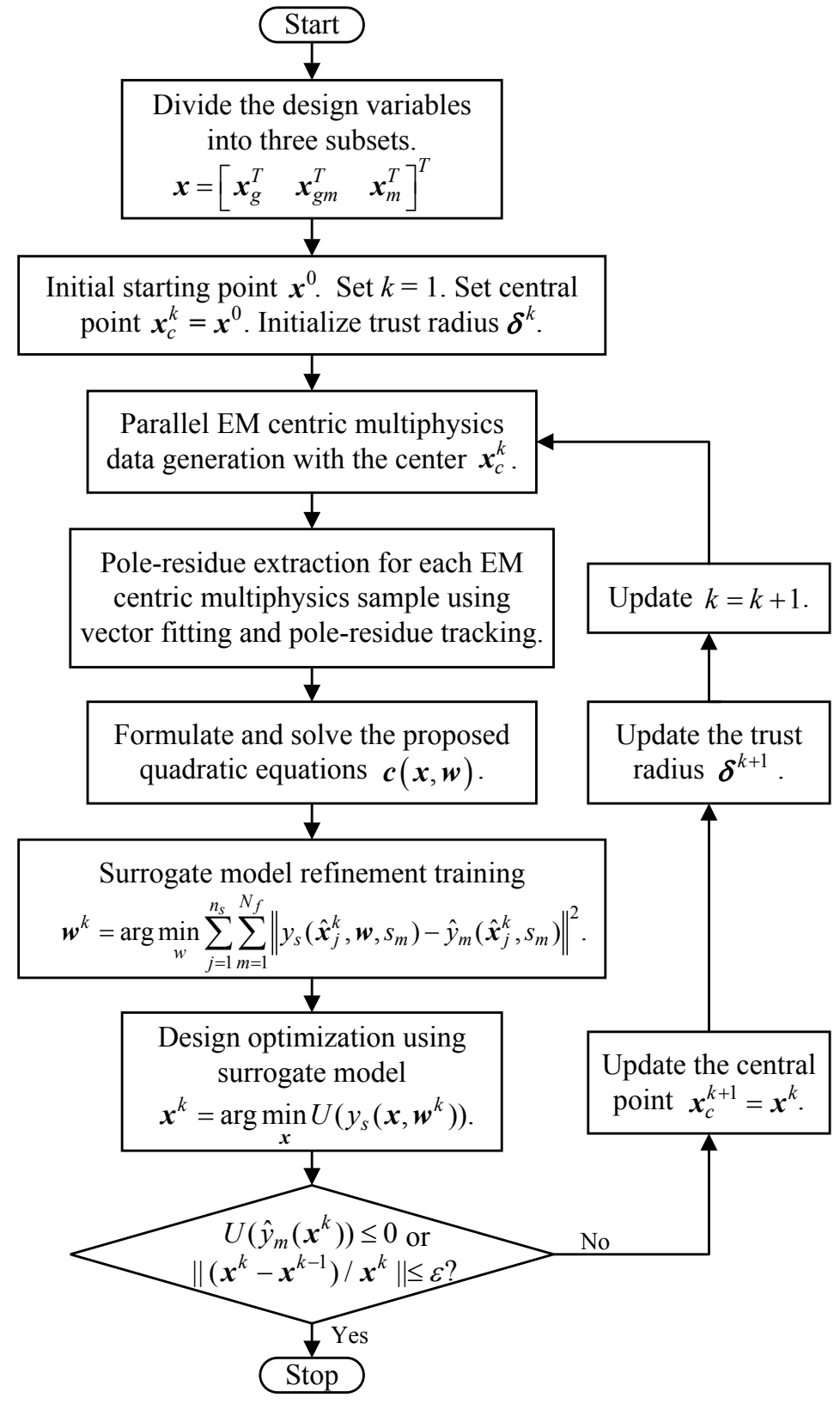

Figure 4.3: Flowchart of the proposed parallel EM centric multiphysics surrogate optimization process. 
$\left\{\widehat{\boldsymbol{x}}_{1}^{k}, \widehat{\boldsymbol{x}}_{2}^{k}, \cdots, \widehat{\boldsymbol{x}}_{n_{s}}^{k}\right\}$ around the central point $\boldsymbol{x}_{c}^{k}$

Step 3) Evaluate multiple EM centric multiphysics responses $\widehat{y}_{m}\left(\widehat{\boldsymbol{x}}_{i}^{k}, s\right), i=1,2, \cdots, n_{s}$, simultaneously in (4.3) using parallel hybrid distributed-shared memory computing platform for all the samples.

Step 4) Extract the poles and residues of the transfer function for each sample using vector fitting technique and pole-residue tracking technique.

Step 5) Preliminary training for the quadratic functions $\boldsymbol{c}_{s}^{(0)}, \boldsymbol{c}_{s}^{(0)}, \cdots, \boldsymbol{c}_{s}^{\left(n_{3}\right)}$ in the surrogate model. The coefficients for the quadratic functions are determined using (4.12) - (4.13).

Step 6) Perform model refinement training for the overall surrogate model $y_{s}$ using $(4.16)-(4.17)$

Step 7) Perform design optimization using surrogate model to find the next optimal surrogate solution $\boldsymbol{x}^{k}$ using (4.18).

Step 8) If one of the termination conditions in (4.21) or (4.22) is satisfied then go to Step 11, else go to next step.

Step 9) Update the trust radius $\boldsymbol{\delta}^{k+1}$ using (4.19) - (4.20).

Step 10) Set the next optimization update (prospective central point) $\boldsymbol{x}_{c}^{k+1}=\boldsymbol{x}^{k}$. Increase the iteration counter $k=k+1$ and go to Step 2 .

Step 11) Stop the optimization process. 


\subsection{Numerical Examples \\ 4.3.1 Multiphysics Optimization of Tunable Evanescent Mode Cavity Filter}

The first example under consideration is the multiphysics optimization of tunable evanescent mode cavity filter. Fig. 5.7 illustrates the structure of this tunable evanescent mode cavity filter [128]. By applying the electric field to the piezo actuator, it can generate a geometrical strain which is proportional to the electric field due to the piezoelectric effect [128]. For this example, the geometrical design variables are the gap $(H)$ between the bottom side of the piezo actuator and the top of the post, the width $(W)$ and the length $(L)$ of the post. The voltage $(V)$ applied to the piezo actuator is the multiphysics design variable, i.e., $\boldsymbol{x}_{m}=[V]$. The added voltage $(V)$ can result in the deformation and displacement of the piezo actuator, subsequently changing the air gap $(H)$ to affect the EM-centric multiphysics response. The width, height, and length of the cavity filter are $a=100 \mathrm{~mm}, b=50$ $\mathrm{mm}$, and $d=50 \mathrm{~mm}$, respectively.

The EM centric multiphysics evaluation is performed by COMSOL multiphysics simulator. Fig. 4.5 shows the practical process of this multiphysics analysis. The deformed structure of the tunable evanescent mode cavity filter with the multi-

physics design variables $\boldsymbol{x}=\left[\begin{array}{lllll}20 & 20 & 170 & -300\end{array}\right]^{T}[\mathrm{~mm} \mathrm{~mm} \mathrm{um} \mathrm{V}]$ is illustrated in Fig. 4.6. It is observed that the piezo actuator can deflect upwards the bottom when the negative voltage is applied.

The desired filter specification is defined as $\left|S_{11}\right| \leq-10 \mathrm{~dB}$ in the frequency ranges $3.098 \mathrm{GHz}$ to $3.102 \mathrm{GHz}$. We select the starting point based on the past 


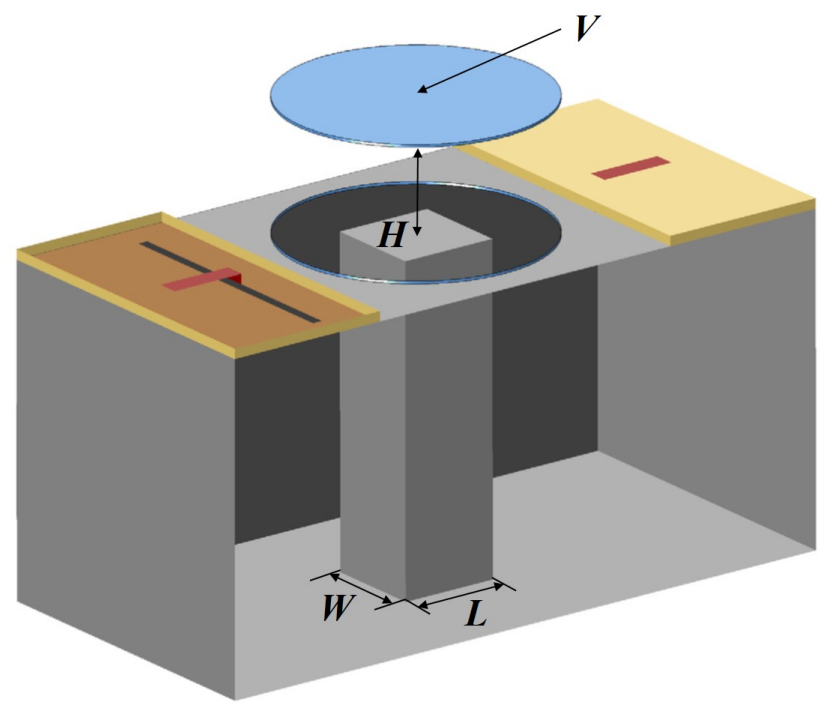

Figure 4.4: The tunable cavity filter structure for multiphysics optimization with four design parameters.

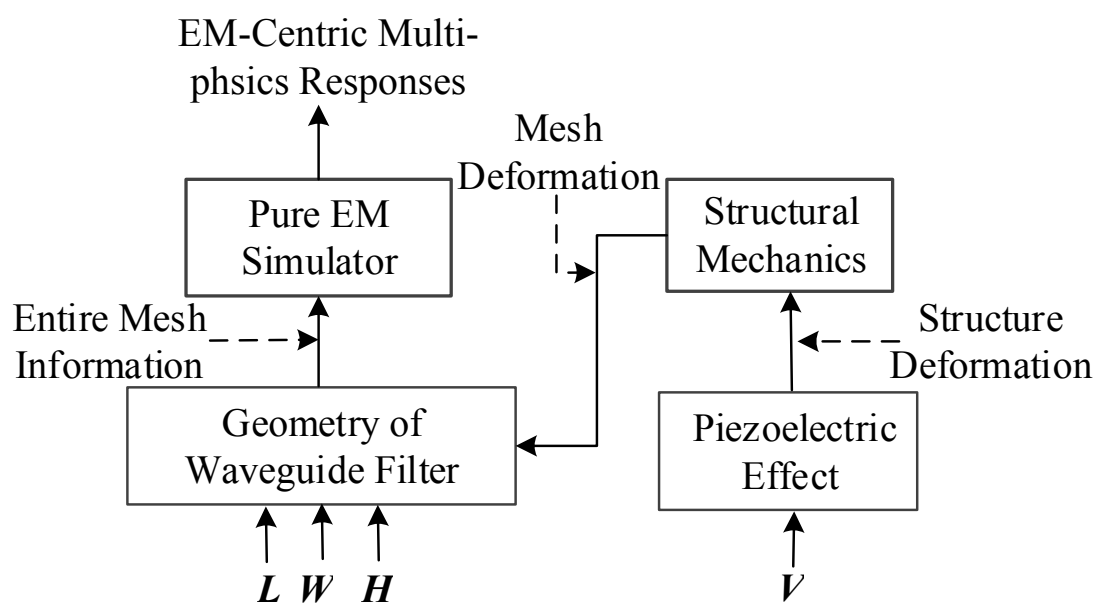

Figure 4.5: The practical process of the multiphysics analysis for the tunable evanescent mode cavity filter example.

design of similar filters. In this tunable cavity filter example, $\boldsymbol{x}^{0}=\left[\begin{array}{llll}20 & 20 & 220 & 0\end{array}\right]^{T}$ $[\mathrm{mm} \mathrm{mm}$ um $\mathrm{V}]$ is selected as the starting point. The neighborhood around the 


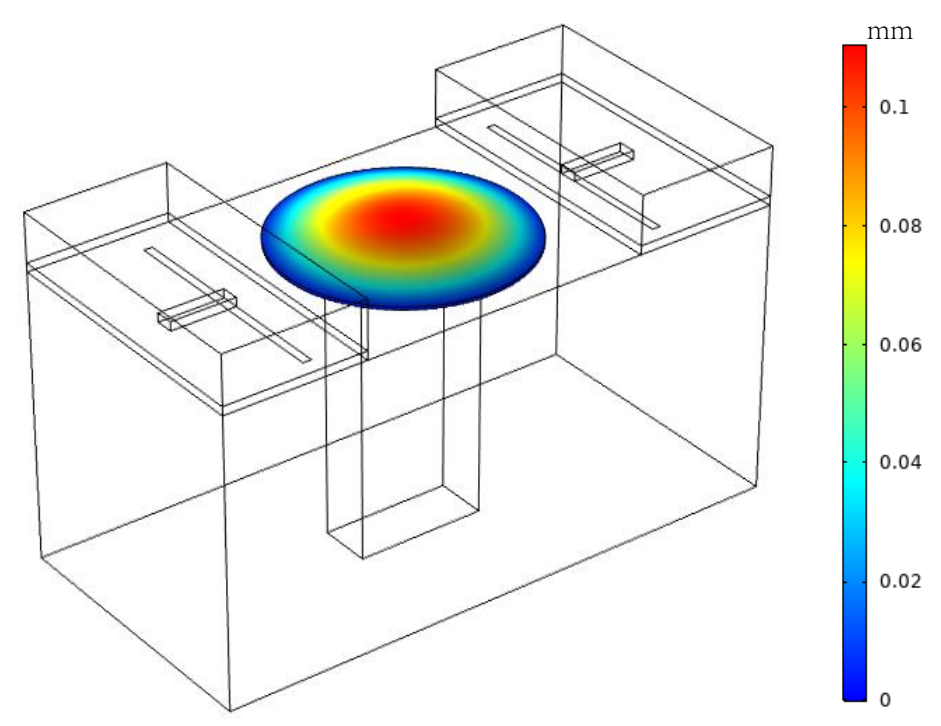

Figure 4.6: The deformed structure of the tunable cavity filter example due to the piezo electric effects.

starting point for $k$ th iteration $X_{s}^{k}$ is defined in $(4.15)$. Here $\delta^{1}(k=1)$ is a user defined initial trust radius selected based on the sensitivity of the multiphysics data and is chosen as $\left[\begin{array}{llll}3 & 3 & 40 & 100\end{array}\right]^{T}[\mathrm{~mm} \mathrm{~mm} \mathrm{um} \mathrm{V}$. We update the trust radius after each iteration using the trust region algorithm in (4.19) - (4.20).

In this example, the multiphysics optimization problem space has four design variables. The multiphysics design variable, i.e., the voltage $(V)$ can affect the multiphysics responses mainly by changing the small gap $(H)$, i.e., $\boldsymbol{x}_{g m}=[H]$ and the $\boldsymbol{x}_{g}=[L W]^{T} . n_{1}, n_{2}$, and $n_{3}$ equal 2, 1, and 1, respectively. Based on our proposed modified quadratic mapping function, the size of unknown weighting vector $\boldsymbol{w}$ in (4.11) is 13 . In order to obtain an accurate surrogate model, the number of training samples should be equal or larger than the number of unknown weighting parameters. Because the number of levels is better to be an odd number to make 
the samples orthogonal to each other, five levels of DOE is used for generating the training samples, i.e., 25 multiphysics training samples. These 25 training samples are distributed through several computers with multiple processors to balance the workload of each processor. We use a cluster of Dell PowerEdge computers with Intel Xeon X5680 processor with each computer having eight processing cores. Using this cluster, 25 different design samples are evaluated in parallel. The structure of the surrogate model which is expressed as a transfer function in pole-residue format is shown in Fig. 4.7. In this example, 4 poles are sufficient to get the accurate vector fitting for the $25 \mathrm{EM}$ centric multiphysics responses. NeuroModerlerPlus software is used to implement the surrogate model training and perform the design optimization using the surrogate model.

Using the proposed technique, the optimal solution $\boldsymbol{x}^{4}=[22.39259 .45923362 .28-$ $359.19]^{T}[\mathrm{~mm} \mathrm{~mm} \mathrm{um} \mathrm{V}]$ is obtained after four iterations. The EM centric multiphysics responses from the initial point and the last iteration are shown in Fig. 4.8 (a) and (b) respectively.

For this tunable evanescent mode cavity filter example, we compare the proposed technique with the direct multiphysics optimization method where the optimization algorithm is applied directly to the multiphysics simulation, which is the benchmark method for multi-physics optimization. For this example, considering the complexity of the EM responses and dimensionality of the design space, we set the number of direct optimization iteration to be 120 . The direct multiphysics optimization does not converge to the optimal solution after 120 iterations (i.e., 120 multiphysics evaluations), whereas our proposed technique takes 4 iterations to reach the opti- 


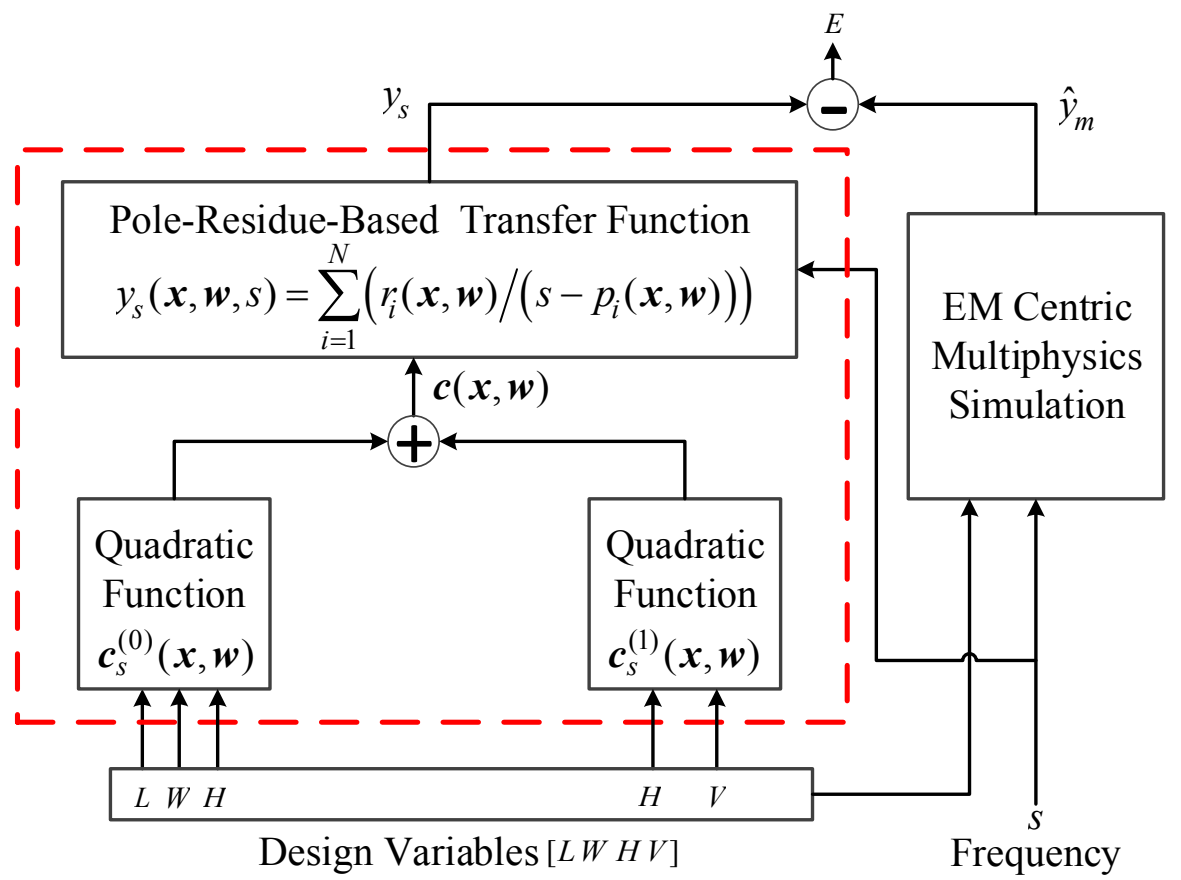

Figure 4.7: The structure of the proposed surrogate model for the cavity filter example.

mal solution. The proposed optimization can converge in fewer iterations to reach the optimal solution compared to the direct multiphysics optimization method for this tunable evanescent mode cavity filter example. The comparisons of the results for the proposed multiphysics optimization technique and the direct multiphysics optimization method is shown in Table 5.9.

The values of the objective function for all the iterations of the proposed multiphysics optimization method are shown in Fig. 4.9. From the figure, we can see that the proposed optimization can converge in four iterations to reach the optimal solution. 


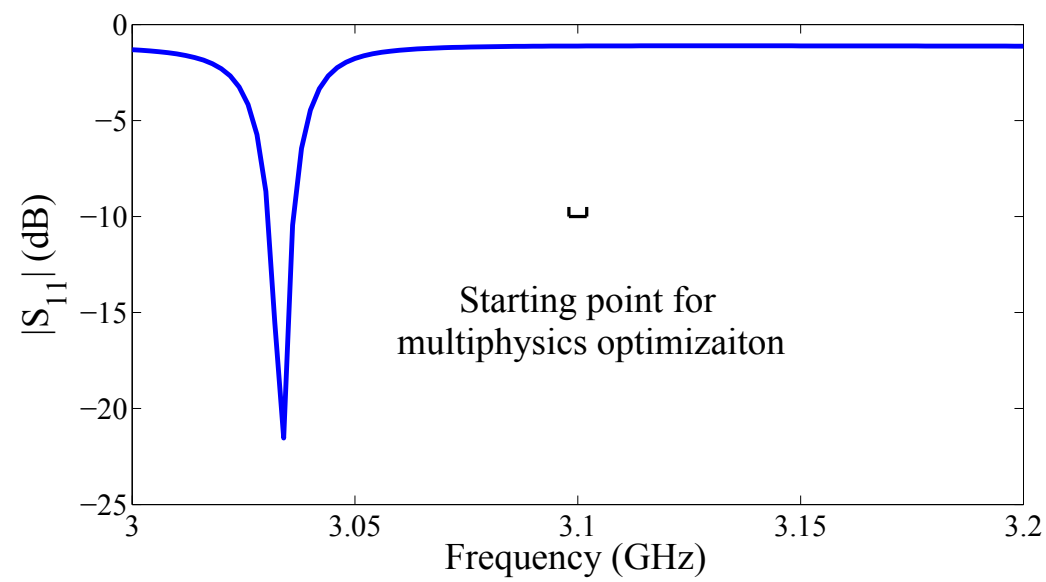

(a)

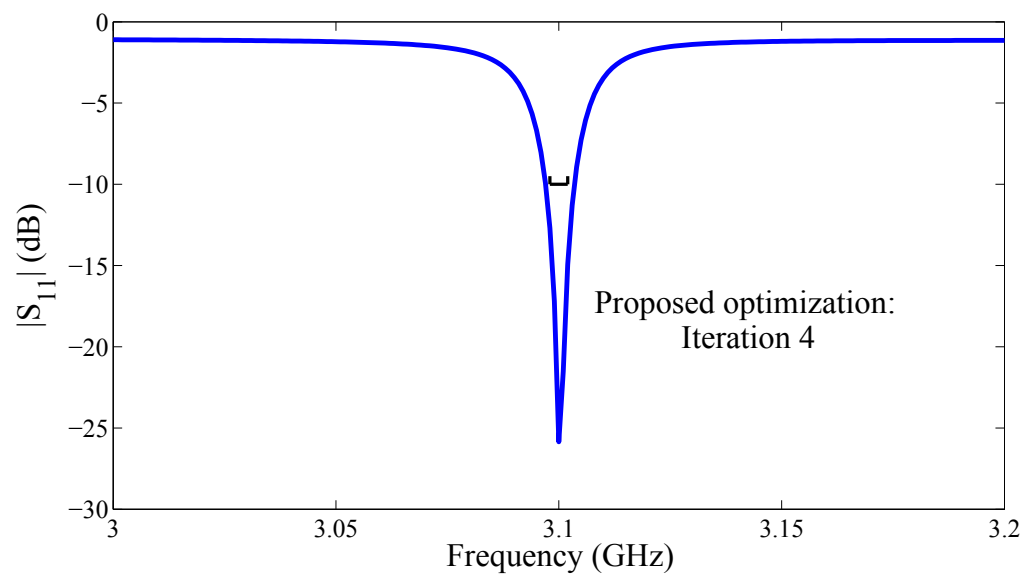

(b)

Figure 4.8: EM centric multiphysics responses for the tunable evanescent mode cavity filter example. (a) Multiphysics response at the starting point. (b) Multiphysics response using the proposed optimization techniques (after four iterations). The interval indicates the design specification for this cavity filter example.

\subsubsection{Multiphysics Optimization of Tunable Four-Pole Waveg- uide Filter Using Piezo Actuator}

The second example under consideration is the multiphysics optimization of a fourpole waveguide filter using the piezo actuator [127]. Fig. 5.12 shows the structure 
Table 4.1: Comparisons of Two Optimization Algorithms for the Tunable Evanescent Mode Cavity Filter Example

\begin{tabular}{|c|c|c|}
\hline $\begin{array}{l}\text { Optimization } \\
\text { Algorithms }\end{array}$ & $\begin{array}{c}\text { Direct Multiphysics } \\
\text { Optimization }\end{array}$ & $\begin{array}{c}\text { Proposed Multiphysics } \\
\text { Optimization }\end{array}$ \\
\hline $\begin{array}{l}\text { No. of Multiphysics } \\
\text { Samples per Iteration }\end{array}$ & 1 & 25 \\
\hline $\begin{array}{c}\text { Multiphysics Evaluation } \\
\text { Time per Iteration }\end{array}$ & $7 \mathrm{~m} 57 \mathrm{~s}$ & $8 \mathrm{~m} 35 \mathrm{~s}$ \\
\hline No. of Iterations & 120 & 4 \\
\hline $\begin{array}{l}\text { Surrogate Model } \\
\text { Training Time }\end{array}$ & - & $5 \mathrm{~m} \times 4$ \\
\hline $\begin{array}{l}\text { Surrogate Model } \\
\text { Optimization Time }\end{array}$ & - & $30 \mathrm{~s} \times 4$ \\
\hline $\begin{array}{c}\text { Multiphysics } \\
\text { Evaluation Time }\end{array}$ & $7 \mathrm{~m} 57 \mathrm{~s} \times 120$ & $8 \mathrm{~m} 35 \mathrm{~s} \times 5$ \\
\hline Total Time & 954 min* & $34.72 \mathrm{~min}$ \\
\hline
\end{tabular}

* Design specifications are not satisfied

of this waveguide filter. The heights $h_{1}$ and $h_{2}$ represent the heights of the tuning posts in the coupling windows. Heights $h_{c 1}$ and $h_{c 2}$ represent the heights of the square cross section which are located at the center of the resonator cavities. The two voltages $V_{1}$ and $V_{2}$ represent the electronic potentials which are supplied to the piezo actuator. The thickness for all the coupling windows in this example is $2 \mathrm{~mm}$.

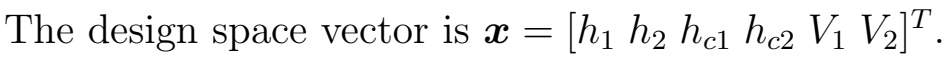




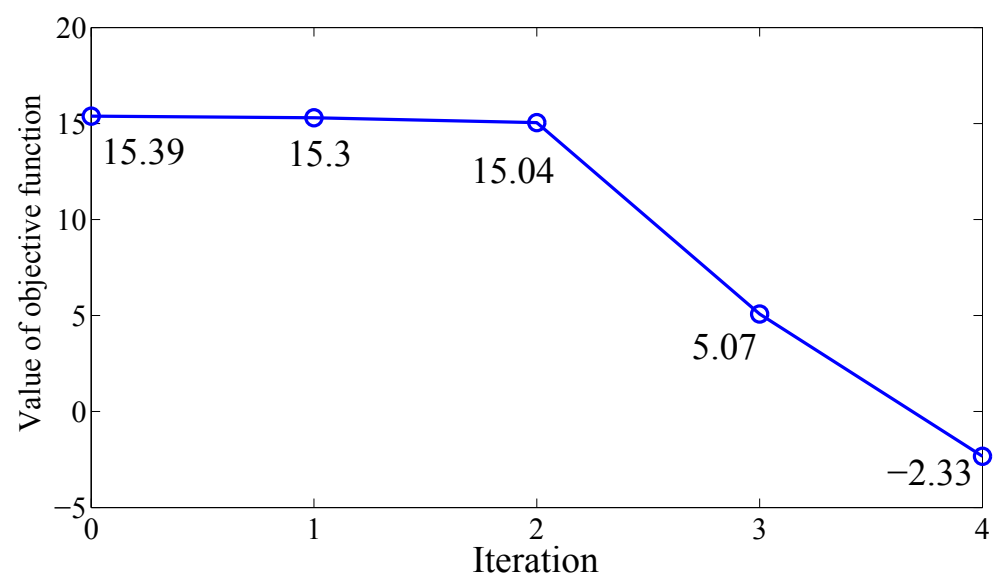

Figure 4.9: Objective function values of the tunable evanescent mode cavity filter example using the proposed multiphysics optimization method. The proposed optimization can converge in four iterations to reach the optimal solution.

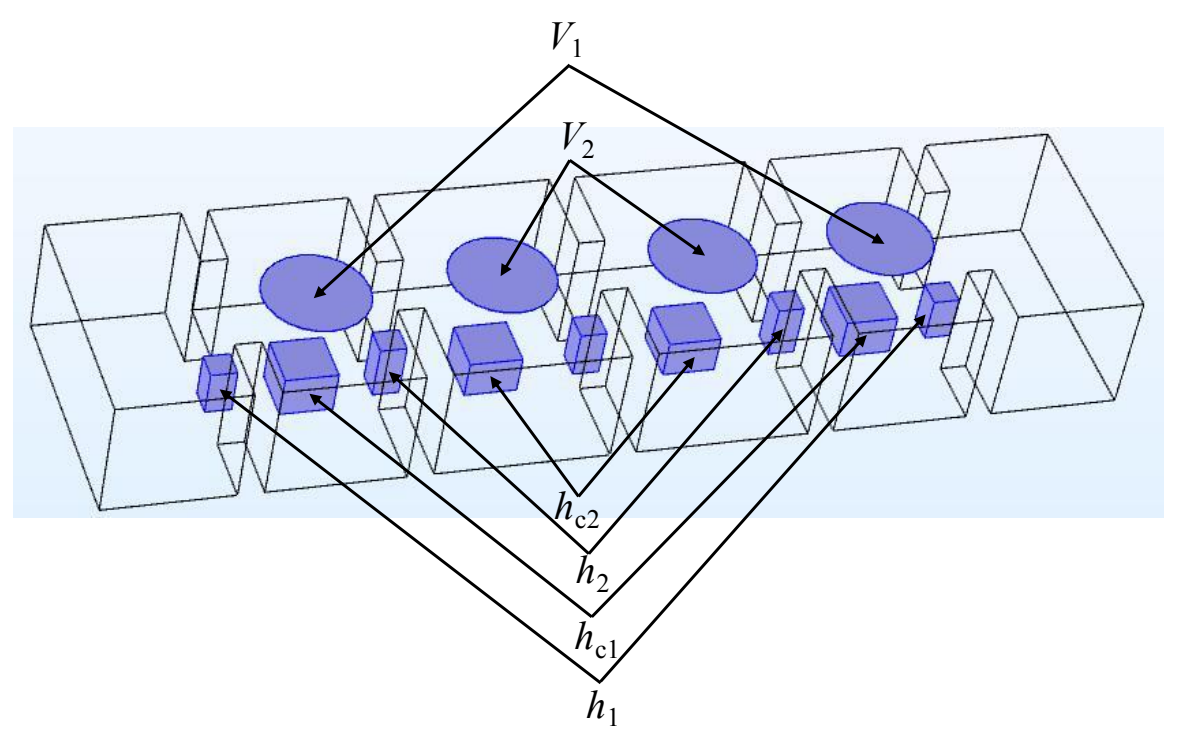

Figure 4.10: Four-pole waveguide filter structure using piezo actuator for multiphysics optimization with six design parameters. 


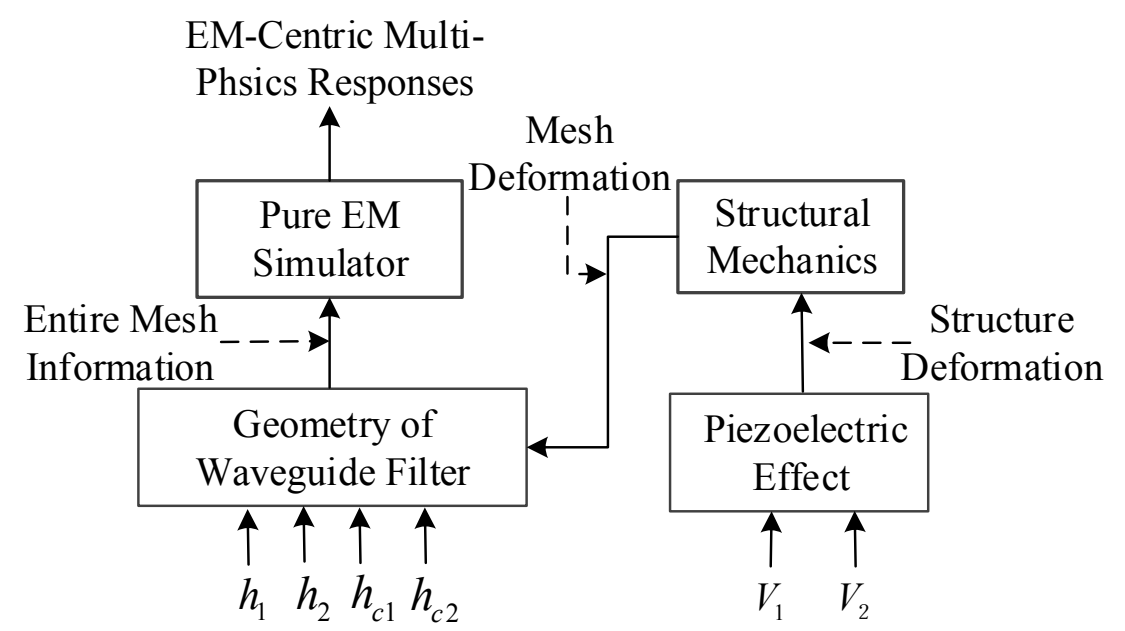

Figure 4.11: The practical process of the multiphysics analysis for the four-pole waveguide filter example.

COMSOL multiphysics simulator is used to perform the EM centric multiphysics evaluations. Fig. 4.11 shows the practical process of this multiphysics analysis. To observe the piezo electric effects, we perform the multiphysics simulation with the

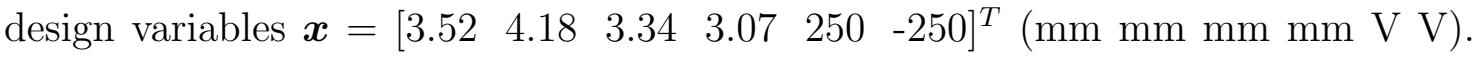
The deformed structure of the four pole waveguide filter is illustrated in Fig. 4.12. From the figure, we can see that with the positive voltage $\left(V_{1}=250 \mathrm{~V}\right)$, the piezo actuator will deflect towards the bottom side, while with the negative voltage $\left(V_{2}\right.$ $=-250 \mathrm{~V})$, the piezo actuator will deflect upwards the bottom side.

The desired filter specification are defined as $\left|S_{11}\right| \leq-24 \mathrm{~dB}$ in the frequency ranges $10.83 \mathrm{GHz}$ to $11.11 \mathrm{GHz}$. We select the starting point based on the past design of similar filters. In this four-pole waveguide filter example, $\boldsymbol{x}^{0}=[3.23 .84$ $\left.\begin{array}{llll}3.02 & 2.72 & 0 & 0\end{array}\right]^{T}[\mathrm{~mm} \mathrm{~mm} \mathrm{~mm} \mathrm{~mm} \mathrm{~V} \mathrm{~V}]$ is selected as the starting point. The neighborhood around the starting point for $k$ th iteration $X_{s}^{k}$ is defined in (4.15). 


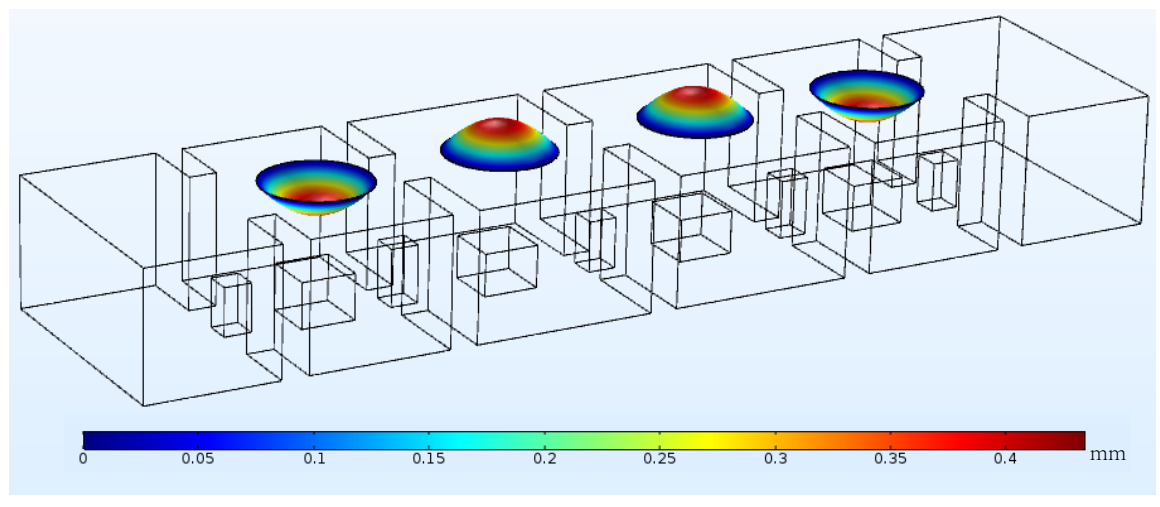

Figure 4.12: The deformed structure of the four pole waveguide filter due to the piezo electric effects.

Here $\delta^{1}(k=1)$ is a user defined initial trust radius selected based on the sensitivity of the EM centric multiphysics data and is chosen as $\left[\begin{array}{llllll}0.08 & 0.08 & 0.08 & 0.08 & 50 & 50\end{array}\right]^{T}$ $[\mathrm{mm} \mathrm{mm} \mathrm{mm} \mathrm{mm} \mathrm{V} \mathrm{V]}$. The trust radius is updated after each iteration using the trust region algorithm in (4.19) - (4.20).

In this example, the multiphysics optimization problem space has six design variables. The multiphysics design variables, i.e., $\boldsymbol{x}_{m}=\left[\begin{array}{ll}V_{1} & V_{2}\end{array}\right]^{T}$ affect the frequency responses mainly by controlling the heights of the square cross section, i.e., $\boldsymbol{x}_{g m}=$ $\left[h_{c 1} h_{c 2}\right]^{T}$ and the $\boldsymbol{x}_{g}=\left[h_{1} h_{2}\right]^{T} . n_{1}, n_{2}$, and $n_{3}$ equal 2, 2, and 2, respectively. Based on our proposed modified quadratic mapping function, the size of unknown weighting vector $\boldsymbol{w}$ in (4.11) is 23, while the traditional quadratic function will result in 28 unknown weighting parameters. Using our proposed technique, the minimum number of training samples is 23 instead of 28 . In this example, we use five levels of DOE for generating the training samples, i.e., 25 multiphysics training samples. These 25 different design samples are evaluated in parallel. The structure of the 
surrogate model which is expressed as a transfer function in pole-residue format is shown in Fig. 4.13. In this example, 12 poles are sufficient to get the accurate vector fitting for the $25 \mathrm{EM}$ centric multiphysics responses. NeuroModerlerPlus software is used to implement the surrogate model training and perform the design optimization using the surrogate model.

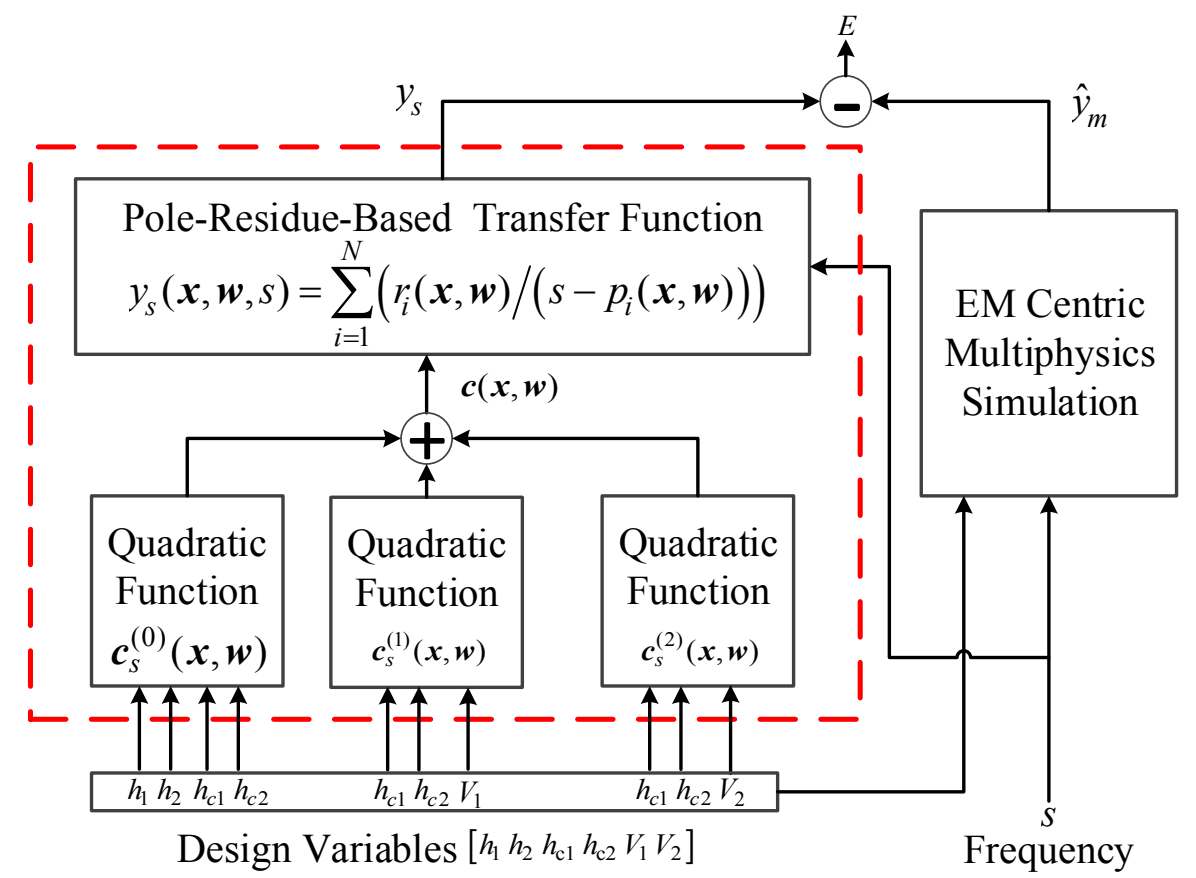

Figure 4.13: The structure of the surrogate model for the tunable evanescent mode cavity filter example.

Using the proposed technique, the optimal solution $\boldsymbol{x}^{5}=[3.465794 .1583 .2187$ $2.94776239 .55270 .156]^{T}[\mathrm{~mm} \mathrm{~mm} \mathrm{~mm} \mathrm{~mm} \mathrm{~V} \mathrm{V]} \mathrm{is} \mathrm{obtained} \mathrm{after} \mathrm{five} \mathrm{iterations.}$ The EM centric multiphysics responses from the initial point and the last iteration are shown in Fig. 4.14 (a) and (b) respectively.

For this four-pole waveguide filter example, we compare the proposed technique 


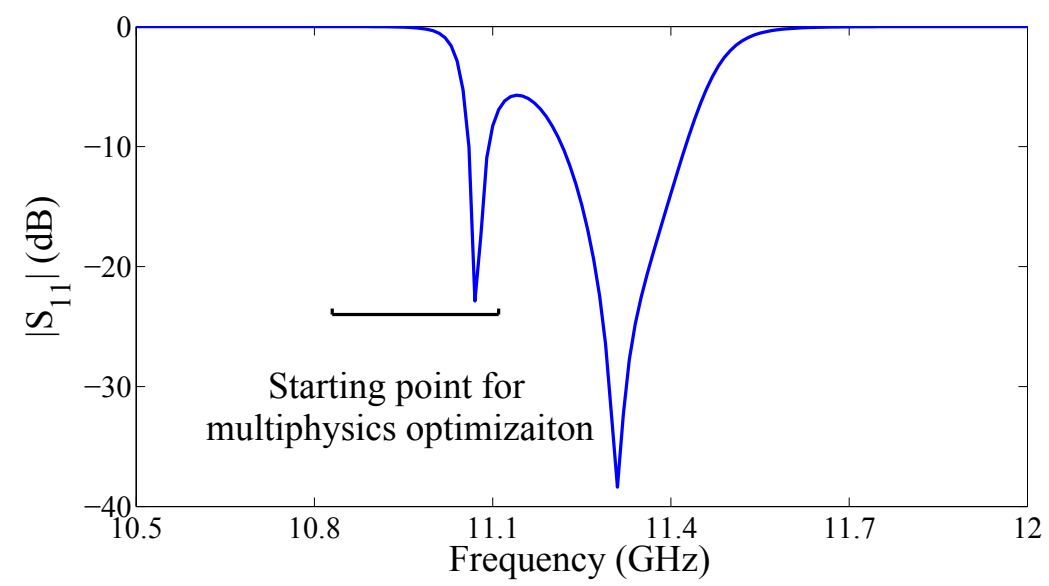

(a)

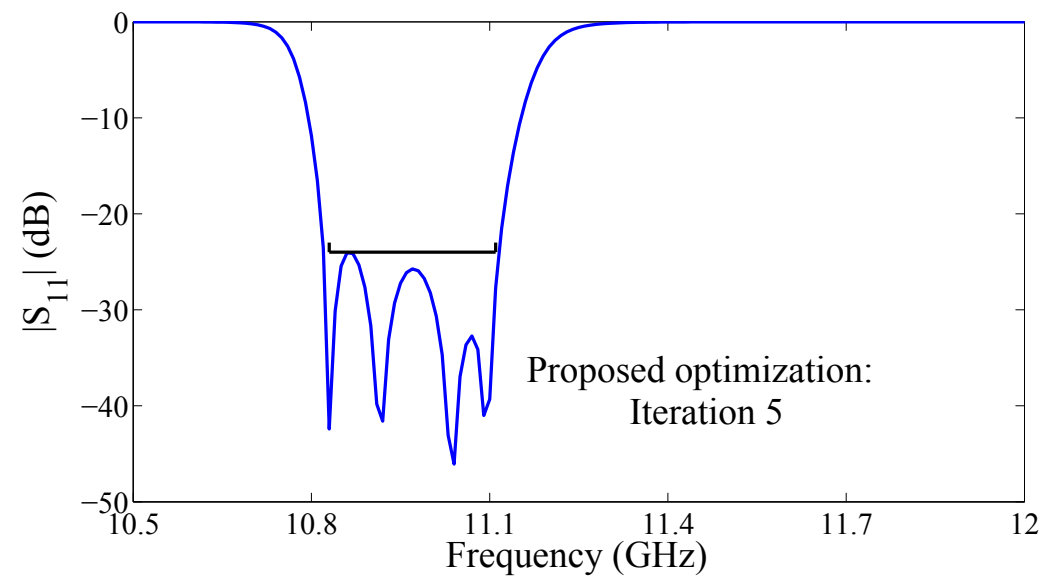

(b)

Figure 4.14: EM centric multiphysics responses for the four-pole waveguide filter example. (a) Multiphysics response at the starting point. (b) Multiphysics response using the proposed optimization techniques (after five iterations). The interval indicates the design specification for this waveguide filter example.

with the direct multiphysics optimization method. The number of direct optimization iteration is set to be 260 considering the complexity of the EM responses and dimensionality of the design space. The direct multiphysics optimization does not 


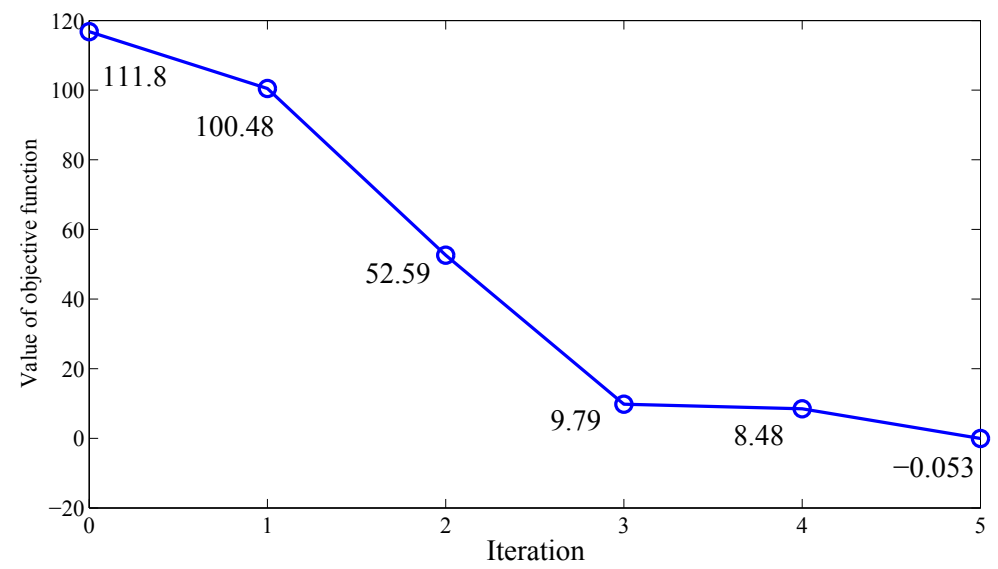

Figure 4.15: Objective function values of the four pole wavaguide filter example using the proposed multiphysics optimization method. The proposed optimization can converge in five iterations to reach the optimal solution.

converge to the optimal solution after 260 iterations (i.e., 260 multiphysics evaluations), whereas our proposed technique takes 5 iterations to the optimal solution. The proposed optimization can converge in fewer iterations to reach the optimal solution compared to the direct multiphysics optimization method for this four-pole waveguide filter example. The comparisons of the results for the proposed multiphysics optimization technique and the direct multiphysics optimization method is shown in Table 5.19.

The values of the objective function for all the iterations of the proposed multiphysics optimization method are shown in Fig. 4.15. From the figure, we can see that the proposed optimization can converge in five iterations to reach the optimal solution. 
Table 4.2: Comparisons of Two Optimization Algorithms for the Four-Pole Waveguide Filter Example

\begin{tabular}{c|c|c}
\hline \hline $\begin{array}{c}\text { Optimization } \\
\text { Algorithms }\end{array}$ & $\begin{array}{c}\text { Direct Multiphysics } \\
\text { Optimization }\end{array}$ & $\begin{array}{c}\text { Proposed Multiphysics } \\
\text { Optimization }\end{array}$ \\
\hline $\begin{array}{c}\text { No. of Multiphysics } \\
\text { Samples per Iteration }\end{array}$ & 1 & 25 \\
\hline $\begin{array}{c}\text { Multiphysics Evaluation } \\
\text { Time per Iteration }\end{array}$ & $20 \mathrm{~m} 25 \mathrm{~s}$ & $23 \mathrm{~m} \mathrm{20 \textrm {s }}$ \\
\hline No. of Iterations & 260 & $5 \mathrm{~m} \times 5$ \\
\hline $\begin{array}{c}\text { Surrogate Model } \\
\text { Training Time }\end{array}$ & - & $30 \mathrm{~s} \times 5$ \\
\hline $\begin{array}{c}\text { Surrogate Model } \\
\text { Optimization Time }\end{array}$ & - & $23 \mathrm{~m} 20 \mathrm{~s} \times 6$ \\
\hline $\begin{array}{c}\text { Multiphysics } \\
\text { Evaluation Time }\end{array}$ & $20 \mathrm{~m} 25 \mathrm{~s} \times 260$ & $2.78 \mathrm{~h}$ \\
\hline Total Time & $88.4 \mathrm{~h}^{*}$ & \\
\hline
\end{tabular}

* Design specifications are not satisfied

\subsection{Conclusion}

In this chapter, we have proposed a novel parallel EM centric multiphysics optimization technique. In the proposed technique, pole-residue based transfer function has been exploited to build an effective and robust surrogate model. A group of modified quadratic mapping functions has been formulated to map the relationships between pole/residues of the transfer function and the design variables. Multiple 
EM centric multiphysics evaluations have been performed in parallel to generate the training data for establishing the surrogate model. The surrogate model is valid in a relatively large neighborhood which makes a large and effective optimization update in each optimization iteration. The trust region algorithm has been adopted to guarantee the convergence of the proposed multiphysics optimization algorithm. Our proposed multiphysics optimization technique has taken a small number of optimization iterations to obtain the optimal EM centric multiphysics response. 


\section{Chapter 5}

\section{Parallel Decomposition Approach for Wide Range Parametric Modeling of Microwave Components}

In this chapter, we propose a novel decomposition technique to address the challenges of EM parametric modeling with geometrical changes in a large range [60]. In this method, a systematic and automated algorithm based on second-order derivative information is proposed to decompose the overall geometrical range into a set of sub-ranges. Using the proposed technique, a smooth region is decomposed into a few large sub-regions while a highly nonlinear region is decomposed into many small sub-regions. The proposed technique provides an efficient mathematical methodology to perform the decomposition systematically and automatically. An artificial neural network (ANN) model with simple structure, hereby referred to as a submodel, is developed with geometrical parameters as variables in each sub-region. When the values of geometrical parameters change from the region of one sub- 
model to another sub-model, the discontinuity of EM responses is observed at the boundary between the adjacent sub-models. There are many sub-model boundaries in the overall model resulting in the complex multi-dimensional discontinuity problem. Thereby the overall model provided by directly combining all the trained sub-models cannot be used for design optimization. A sub-model modification process is proposed to solve this multi-dimensional discontinuity problem to obtain a continuous model over the entire region. Parallel data generation, parallel submodel training, and parallel sub-model modification are proposed to speed up the model development process. Compared with standard modeling methods using a single model to cover the entire wide geometrical range, the proposed method can obtain better model-accuracy with short model-development time.

\section{$5.1 \quad$ Introduction}

As a further development of parametric modeling, we consider a more challenging issue in parametric modeling, i.e., wide range modeling. For the design of microwave components with various specifications, the geometrical parameters need to be explored in a large range. However, developing an accurate and efficient parametric model with geometrical changes in a large range is still a challenge. This is because the complexity and non-linearity increase very fast with the increase of the geometrical parameter range. The standard parametric model using one model to cover the entire wide geometrical ranges requires a highly complex model structure to learn the input-output relationship properly. A large amount of CPU time is consumed on the model training process. Various techniques have been introduced to reduce 
the cost of establishing parametric models. A parallel automatic model generation technique is proposed in [32], using parallel adaptive sampling and parallel data generation to save the model development time. To make neural network training more efficient, the automated model generation (AMG) [129], [130] using combined neural network and interpolation techniques is introduced to determine the suitable number of hidden neurons. However, these techniques are not sufficient to solve the challenges of developing the parametric model with a wide geometrical range.

In this chapter, we propose an efficient parallel decomposition technique for parametric modeling where the values of geometrical parameters change in a large range. In this technique, a systematic algorithm based on derivative information is proposed to decompose the overall geometrical range into a set of sub-ranges. Using the proposed technique, a smooth region is decomposed into a few large sub-regions while a highly nonlinear region is decomposed into many small sub-regions. An artificial neural network model with simple structure is developed with geometrical parameters as variables in each sub-region. In this paper, a parametric model (i.e., the ANN model) for a sub-region is called as a sub-model. A parametric model for the entire region is called as an overall model. When the values of geometrical parameters change from the region of one sub-model to another sub-model, the discontinuity of EM responses is observed at the boundary between the adjacent sub-models. Given the specific value of the geometrical parameter which is located at the boundary between the adjacent sub-models, the responses of the adjacent sub-models are very similar but not necessarily equal. The responses are very similar because the adjacent sub-models are trained with same boundary data and are 
well trained in their own sub-range. The responses are not necessarily equal because the adjacent sub-models are separate sub-models and are trained separately. There are many sub-model boundaries in the overall model resulting in the complex multi-dimensional discontinuity problem. Thereby the overall model provided by directly combining all the trained sub-models cannot be used for design optimization. A new algorithm is proposed to solve the multi-dimensional discontinuity problem occurring at the boundary between the adjacent sub-models to obtain a continuous overall model over the entire region. Parallel data generation, parallel sub-model training, and parallel sub-model modification are used to speed up the model development process. The proposed method can obtain better accuracy with faster modeling development time compared with standard modeling methods using a single model to cover the entire wide geometrical ranges.

\subsection{Proposed Decomposition Technique for Wide Range Parametric Modeling}

\subsubsection{Description of Wide Range Problem}

Let $\boldsymbol{x}$ represent a vector containing the geometrical design parameters of a microwave component, defined as

$$
\boldsymbol{x}=\left[\begin{array}{llll}
x_{1} & x_{2} & \cdots & x_{N}
\end{array}\right]^{T}
$$

where $N$ is the number of geometrical parameters for the parametric model. Let $i$ represent the index number of the geometrical parameters, i.e., $i \in\{1,2, \cdots, N\}$. 
Let $\boldsymbol{X}$ be a matrix representing the wide geometrical range, defined as

$$
\boldsymbol{X}=\left[\begin{array}{cc}
X_{1}^{\min } & X_{1}^{\max } \\
X_{2}^{\min } & X_{2}^{\max } \\
\vdots & \vdots \\
X_{N}^{\min } & X_{N}^{\max }
\end{array}\right]
$$

where $X_{i}^{\min }$ and $X_{i}^{\max }$ represent the minimum and maximum values of the geometrical parameter $x_{i}$, respectively. Let $D_{i}$ represent the range of the geometrical parameter $x_{i}$, defined as

$$
D_{i}=X_{i}^{\max }-X_{i}^{\min }, \quad i \in\{1,2, \cdots, N\}
$$

Let $R_{f}(\boldsymbol{x}, f)$ represent the EM output response from the EM simulation in the range of $\boldsymbol{X}$ where $f$ represents the frequency parameter. When the geometrical range is relatively small, we can build an accurate parametric model using the existing techniques. When the geometrical range is relatively large, the relationship between the EM response and the design parameters becomes very complex and highly nonlinear. Developing an accurate overall parametric model that covers a wide geometrical parameter range to represent this highly nonlinear relationship is challenging. We investigate the decomposition technique for the wide range problem. The decomposition is performed in the multi-dimensional geometrical space and a large number of sub-models is generated, which makes the decomposition process very expensive and cumbersome to be done manually. We propose an efficient mathematical methodology to perform the decomposition so that the cumbersome 
decomposition process can be done systematically and automatically.

For microwave components, the sensitivity of EM response may vary w.r.t. different geometrical parameters and different regions of geometrical parameters. we propose to incorporate the second-order derivatives of the EM response w.r.t. the geometrical variables to guide the decomposition process. Using the proposed technique, we decompose the modeling problem with a wide geometrical range into a set of sub-range modeling problems where a smooth region is decomposed into a few large sub-regions while a highly nonlinear region is decomposed into many small sub-regions.

\subsubsection{Formulation of Second-Order Derivative}

To build an accurate parametric model, a suitable sampling method should be determined. The full-grid distribution sampling method [17] is feasible when the number of design variables $N$ is small and the number of levels for each design variable is small. However, when the number of design variables or the number of levels becomes larger, the full-grid distribution leads to an exponential increase of sample points. For the wide range problem, the number of levels using the grid distribution is large which may require many EM evaluations. For randomly distributed data generation method, the total number of samples is dictated by user applications. In our proposed technique, we use a randomly distributed data generation method to generate enough training data and testing data in the defined wide range $\boldsymbol{X}$ to represent the highly nonlinear input-output relationships, at the same time avoid the exponentially large amount of data in grid distribution. The 
number of training samples is defined as $N_{s}$. Let $k$ represent the index number of the training samples, i.e., $k \in\left\{1,2, \cdots, N_{s}\right\}$. Parallel computational approaches are implemented to accelerate the EM data generation process.

Let $g_{i}{ }^{k}$ be the first order derivative of EM response w.r.t. the geometrical parameter $x_{i}$ at the $k^{\text {th }}$ training sample, represented as

$$
g_{i}^{k}=\frac{\partial R_{f}\left(\boldsymbol{x}^{k}, f\right)}{\partial x_{i}}
$$

$g_{i}{ }^{k}$ can be obtained directly from EM simulator. Let $\boldsymbol{g}^{k}$ represent a vector containing all the values of $g_{i}{ }^{k}$ for $i=1,2, \cdots, N$. Sensitivity is evaluated at each training sample $k$, where $k=1,2, \cdots, N_{s}$. Let $H_{i, i}^{k}$ be the second-order derivative of the EM response w.r. t. the geometrical parameter $x_{i}$ for the $k^{\text {th }}$ training sample, represented as

$$
H_{i, i}^{k}=\frac{\partial g_{i}^{k}}{\partial x_{i}}=\frac{\partial^{2} R_{f}\left(\boldsymbol{x}^{k}, f\right)}{\left(\partial x_{i}\right)^{2}}
$$

The second-order derivative is not available explicitly from the EM simulator. Since the training data is generated using the randomly distributed sampling method, we cannot compute the second-order derivative using perturbation techniques such as the finite difference method. We propose to use the EM responses and first order EM derivatives at several samples around the $k^{\text {th }}$ sample to derive the second order EM derivatives at the $k^{\text {th }}$ training sample.

The desired second-order derivative information $H_{i, i}^{k}$ are the diagonal elements of the Hessian matrix [131] at the $k^{\text {th }}$ training sample. Let $\boldsymbol{H}^{k}$ represent the Hessian matrix at the $k^{t h}$ training sample containing all of elements of $H_{i, j}^{k}, i, j \in$ $\{1,2, \cdots, N\}$ where $H_{i, j}^{k}$ represents the second-order derivative w.r.t. geometrical 
parameters $x_{i}$ and $x_{j}$. If we can obtain $\boldsymbol{H}^{k}$, we can get the desired second-order derivative information $H_{i, i}^{k}$. Let $N_{u}$ represent the number of unknown elements in the Hessian matrix. The calculation of the value of $N_{u}$ is provided in Appendix A.

Let $\boldsymbol{h}^{k}$ be a vector containing all the unknown elements in the Hessian matrix at the $k^{\text {th }}$ training sample, represented as

$$
\boldsymbol{h}^{k}=\left[\begin{array}{llllll}
H_{1,1}^{k} & H_{1,2}^{k} & \cdots & H_{i, j}^{k} & \cdots & H_{N, N}^{k}
\end{array}\right]^{T}
$$

where $i \in\{1,2, \cdots, N\}$ and $j \in\{i, i+1, \cdots, N\}$. To obtain $\boldsymbol{h}^{k}$ which contains the $N_{u}$ unknown elements in the Hessian matrix, we need at least $N_{u}$ samples which are close to $\boldsymbol{x}^{k}$. In our proposed technique, to obtain the accurate second-order derivative, we select $N_{h}$ samples where $N_{h}$ is the number of training samples closest to $\boldsymbol{x}^{k}$. The value of $N_{h}$ is user-defined and larger than the value of $N_{u}$. To obtain the $N_{h}$ samples, the first step is to calculate the distance between $\boldsymbol{x}^{k}$ and the other $N_{s}-1$ training samples. Let $O_{k, m}$ represent the distance between $\boldsymbol{x}^{k}$ and another training sample $\boldsymbol{x}^{m}$, formulated as

$$
O_{k, m}=\sqrt{\sum_{i=1}^{N}\left(x_{i}^{k}-x_{i}^{m}\right)^{2} /\left(X_{i}^{\max }-X_{i}^{\min }\right)^{2}}, \quad \forall m, m \neq k .
$$

The second step is to reorder $O_{k, m}$ for $\forall m, m \neq k$, in ascending order to find the first $N_{h}$ samples that are closest to $\boldsymbol{x}^{k}$. Let $\boldsymbol{I}_{k}$ be an index set containing the indices of the $N_{h}$ samples, defined as

$$
\boldsymbol{I}_{k}=\left\{v \mid \text { if } \boldsymbol{x}^{v} \text { is one of the first } N_{h} \text { samples closest to } \boldsymbol{x}^{k}\right\}
$$


where $1 \leq v \leq N_{s}$. The second-order derivatives at sample $k$, i.e., $\boldsymbol{h}^{k}$, are evaluated by using the information from the $N_{h}$ samples, formulated as

$$
\boldsymbol{h}^{k}=\left(\left(\boldsymbol{A}^{k}\right)^{T} \boldsymbol{A}^{k}\right)^{-1}\left(\boldsymbol{A}^{k}\right)^{T} \boldsymbol{b}^{k}
$$

where $\boldsymbol{A}^{k}$ is a $N_{h} \times N_{u}$ matrix and $\boldsymbol{b}^{k}$ is a $N_{h} \times 1$ vector. The row corresponding to sample $v$ in $\boldsymbol{A}^{k}$ is represented as

$$
\boldsymbol{a}^{k, v}=\left[\begin{array}{llll}
a_{1,1}^{k, v} & a_{1,2}^{k, v} & \cdots & a_{i, j}^{k, v} \cdots \\
\cdots & a_{N, N}^{k, v}
\end{array}\right]
$$

where $a_{i, j}^{k, v}$ is given by

$$
a_{i, j}^{k, v}=\left\{\begin{array}{l}
\frac{1}{2}\left(x_{i}^{v}-x_{i}^{k}\right)^{2}, i=j \\
\left(x_{i}^{v}-x_{i}^{k}\right)\left(x_{j}^{v}-x_{j}^{k}\right), i \neq j
\end{array}\right.
$$

for $i \in\{1,2, \cdots, N\}$ and $j \in\{i, i+1, \cdots, N\}$. The element corresponding to sample $v$ in $b^{k}$ is represented as

$$
b^{k, v}=R_{f}\left(\boldsymbol{x}^{v}, f\right)-R_{f}\left(\boldsymbol{x}^{k}, f\right)-\left(\boldsymbol{g}^{k}\right)^{T}\left(\boldsymbol{x}^{v}-\boldsymbol{x}^{k}\right) .
$$

The detailed derivation of Equation (5.9) is given in Appendix B. Then we can obtain $H_{i, i}^{k}$ as

$$
H_{i, i}^{k}=h_{z}^{k}
$$

where $h_{z}^{k}$ is the $z^{t h}$ element in $\boldsymbol{h}^{k}$ and $z=\frac{(2 N+2-i)(i-1)}{2}+1$. 


\subsubsection{Proposed Decomposition Technique Using Second-Order Derivative}

After obtaining the second-order derivatives for all the training samples, we can perform the decomposition. An efficient mathematical methodology is proposed to perform the decomposition systematically and automatically. Let $N_{f}$ represent the

number of frequency points. Let $H_{i, i, q}^{k}$ be the second-order derivative w.r.t. the geometrical parameter $x_{i}$ at the $k^{\text {th }}$ training sample and the $q^{\text {th }}$ frequency point where $q=1,2, \cdots, N_{f}$. By solving Equation (5.9) at the $q^{\text {th }}$ frequency point, $H_{i, i, q}^{k}$ can be obtained. Let $P_{i}$ denote the total value of the second-order derivatives w.r.t. $x_{i}$ at all training samples, represented as

$$
P_{i}=\sum_{k=1}^{N_{s}} \sum_{q=1}^{N_{f}}\left\|H_{i, i, q}^{k}\right\|=\sum_{k=1}^{N_{s}} \sum_{q=1}^{N_{f}}\left\|\frac{\partial^{2} R_{f}\left(\boldsymbol{x}^{k}, f_{q}\right)}{\left(\partial x_{i}\right)^{2}}\right\| .
$$

Let $Q_{i}$ denote the average value of the second-order derivatives w.r.t. $x_{i}$ at all training samples, represented as

$$
Q_{i}=P_{i} / N_{s}
$$

Let $T_{i}$ denote the average variation of sensitivity of EM response w.r.t. $x_{i}$ at the range $D_{i}$ defined in Equation (5.3), represented as

$$
T_{i}=Q_{i} \cdot D_{i}
$$

For microwave components, the sensitivity of EM response may vary w.r.t. different geometrical parameters. Let $\boldsymbol{T}$ be a vector representing the average variation of the 
sensitivity of EM response w.r.t. all the geometrical parameters, defined as

$$
\boldsymbol{T}=\left[\begin{array}{llll}
T_{1} & T_{2} & \cdots & T_{N}
\end{array}\right]^{T}
$$

Let $T_{\min }$ represent the minimum value in the vector $\boldsymbol{T}$, defined as

$$
T_{\min }=\min _{i \in\{1,2, \cdots, N\}}\left\{T_{i}\right\}
$$

Let $\boldsymbol{L}$ be a vector representing the number of divisions (i.e., number of segments) for all the geometrical parameters, defined as

$$
\boldsymbol{L}=\left[\begin{array}{llll}
L_{1} & L_{2} & \cdots & L_{N}
\end{array}\right]^{T}
$$

where $L_{i}$ is the number of divisions for the geometrical parameter $x_{i}$, determined by

$$
L_{i}=\left\lfloor K \cdot \frac{T_{i}}{T_{\min }}\right\rfloor=\left\lfloor\frac{K}{T_{\min }} \frac{D_{i}}{N_{s}} \sum_{k=1}^{N_{s}} \sum_{q=1}^{N_{f}}\left\|\frac{\partial^{2} R_{f}\left(\boldsymbol{x}^{k}, f_{q}\right)}{\left(\partial x_{i}\right)^{2}}\right\|\right\rfloor
$$

where the symbol \lfloor\rceil means the round value and $K$ represents the stage index which is set to 1 at the first training stage. The proposed technique takes several training stages to obtain an accurate and continuous overall model. If the overall region is smooth, we need a small number of training stages (i.e., small number of divisions) to obtain the overall model. If the overall region is highly nonlinear, we need large number of training stages (i.e., large number of divisions) to obtain the overall model. Based on Equation (5.20), the ranges of geometrical parameters to which the EM response is less sensitive are decomposed into a small number of divisions. Conversely, the ranges of geometrical parameters to which the EM response is more 
sensitive are decomposed into a large number of divisions.

At each training stage, we perform the decomposition for all the geometrical parameters, train the decomposed sub-models using the parallel training approach, and combine the developed sub-models into an overall model. Once we get the overall model, we calculate the overall model training and testing errors using the entire training and testing data. If the testing error is lower than a user-defined threshold $\varepsilon$, the training process terminates and the overall wide range model is developed. Otherwise, we need to start a new stage to re-decompose the entire range into more sub-ranges, i.e., more divisions for each geometrical parameter. We set $K=K+1$ and update the division vector $\boldsymbol{L}$ (i.e., increase the number of sub-regions) using Equation (5.20) to start a new training stage.

After the division vector $\boldsymbol{L}$ is determined at each training stage, we can perform the decomposition to obtain the sub-range of each division for each geometrical parameter at this training stage. Second-order derivative information is incorporated to determine the sub-range so that a smooth region is decomposed into a few large sub-regions while a highly nonlinear region is decomposed into many small sub-regions. In our proposed technique, based on different number of divisions for each geometrical parameter and the second-order derivative information, the entire range $\boldsymbol{X}$ is decomposed into sub-ranges dimension by dimension, i.e., from $x_{1}$ to $x_{N}$. Let the sub-regions obtained from decomposing $x_{i}$ be defined as the $i^{\text {th }}$ level subregions. Before decomposing the $i^{\text {th }}$ geometrical parameter $x_{i}$, several sub-regions have already been obtained because the decomposition of $x_{1}$ to $x_{i-1}$ has already been performed. When decomposing the $i^{\text {th }}$ geometrical parameter $x_{i}$, the $(i-1)^{t h}$ 
level sub-regions are decomposed into $i^{\text {th }}$ level sub-regions along the direction of $x_{i}$. Let $n$ represent the total number of the $i^{\text {th }}$ level sub-regions, calculated as

$$
n=\prod_{j=1}^{i} L_{j}
$$

The total number of the $(i-1)^{t h}$ level sub-regions is $n / L_{i}$. Let $t$ represent the index number of the $(i-1)^{t h}$ level sub-regions, i.e., $t=1,2, \cdots, n / L_{i}$. Let $\boldsymbol{X}^{t}$ be a matrix representing the range of the $t^{t h}$ sub-region, defined as

$$
\boldsymbol{X}^{t}=\left[\begin{array}{cc}
X_{1}^{t, \min } & X_{1}^{t, \max } \\
\vdots & \vdots \\
X_{i}^{t, \min } & X_{i}^{t, \max } \\
\vdots & \vdots \\
X_{N}^{t, \min } & X_{N}^{t, \max }
\end{array}\right] .
$$

We decompose the $(i-1)^{t h}$ level sub-regions into the $i^{\text {th }}$ level sub-regions. The number of the $i^{\text {th }}$ level sub-regions decomposed from sub-region $t$ at the $(i-1)^{t h}$ level is $L_{i}$. The range $\boldsymbol{X}^{t}$ is decomposed into $\boldsymbol{X}_{\text {new }}^{t_{1}}, \boldsymbol{X}_{\text {new }}^{t_{2}}, \cdots, \boldsymbol{X}_{\text {new }}^{t_{p}}, \cdots, \boldsymbol{X}_{\text {new }}^{t_{L_{i}}}$ where $t_{p}=(t-1) L_{i}+p, p \in\left\{1,2, \cdots L_{i}\right\}$ and $\boldsymbol{X}_{\text {new }}^{t_{p}}$ is a matrix representing the range for the $i^{\text {th }}$ level sub-region $t_{p}$, defined as

$$
\boldsymbol{X}_{\text {new }}^{t_{p}}=\left[\begin{array}{cc}
X_{1, \text { new }}^{t_{p} \text { min }} & X_{1, \text { new }}^{t_{p}, \text { max }} \\
\vdots & \vdots \\
X_{i, \text { new }}^{t_{p} \text { min }} & X_{i, \text { new }}^{t_{p}, \text { max }} \\
\vdots & \vdots \\
X_{N, \text { new }}^{t_{p} \text {,in }} & X_{N, \text { new }}^{t_{p}, \text { max }}
\end{array}\right]
$$


Let $N_{t}$ represent the number of training samples that lie in the range of $\boldsymbol{X}^{t}$. To obtain $\boldsymbol{X}_{\text {new }}^{t_{p}}$, we first count the number of training samples that lie in the range of $\boldsymbol{X}^{t}$, i.e., $N_{t}$. Then we reorder the $N_{t}$ training samples according to their value of $x_{i}$ in ascending order. Let $\hat{\boldsymbol{x}}^{k}\left(k \in\left\{1,2, \cdots, N_{t}\right\}\right)$ denote the reordered training samples. Let $C_{i}^{t}$ represent the total value of the second-order derivatives w.r.t. $x_{i}$ at the $N_{t}$ training samples in sub-region $t$, formulated as

$$
C_{i}^{t}=\sum_{k=1}^{N_{t}} \sum_{q=1}^{N_{f}}\left\|\frac{\partial^{2} R_{f}\left(\hat{\boldsymbol{x}}^{k}, f_{q}\right)}{\left(\partial x_{i}\right)^{2}}\right\|
$$

The value of $X_{j \text {,new }}^{t_{p} \text {,min }}$ is calculated using the reordered training samples based on second-order derivative information w.r.t. $x_{i}$, formulated as

$$
X_{j, \text { new }}^{t_{p} \text { min }}= \begin{cases}X_{j}^{t, \text { min }}, & j \neq i \\ X_{j}^{t, \text { min }}, & j=i, p=1 \\ V_{j}^{r}, & j=i, p>1\end{cases}
$$

where $V_{j}^{r}$ is the $j^{\text {th }}$ element of vector $\hat{\boldsymbol{x}}^{r}$ and $r$ is determined by the formula

$$
r=\arg \min _{\gamma \in\left\{1,2, \cdots, N_{t}\right\}}\left\|\sum_{k=1}^{\gamma} \sum_{q=1}^{N_{f}}\right\| \frac{\partial^{2} R_{f}\left(\hat{\boldsymbol{x}}^{k}, f_{q}\right)}{\left(\partial x_{i}\right)^{2}}\left\|-(p-1) C_{i}^{t} / L_{i}\right\| .
$$

Based on Equation (5.26), in a smooth region where the second-order derivative $H_{i, i, q}$ is small, the value of $r$ in that region should be large. Conversely in a highly nonlinear region where the second-order derivative $H_{i, i, q}$ is large, the value of $r$ in that region should be small. Since the training data are generated using a randomly distributed data generation method, the data is distributed uniformly along the di- 
mension of $x_{i}$. Therefore, large $r$ means large region and small $r$ means small region. By using Equation (5.26), the smooth region i.e., the region with small second-order derivative can be large while the highly nonlinear region i.e., the region with large second-order derivative can be small. If there is no overlapping region, the $X_{i \text {,new }}^{t_{p} \text {,min }}$ should be the $X_{i, \text { new }}^{t_{p+1} \text { max }}$. In our proposed technique, in order to achieve an accurate and continuous overall model, we propose to have an overlapping region for the adjacent sub-regions along the dimension of each geometrical parameter. By using overlapping, after the sub-model training process, the adjacent sub-models have very similar output responses at the boundary. This can reduce the gap between the two adjacent sub-models at the boundary which will be discussed in later sections. In our proposed technique, we define $\delta$ as a user-defined overlapping percentage. The value of $X_{j \text {,new }}^{t_{p} \text { max }}$ is formulated as

$$
X_{j, \text { new }}^{t_{p} \text { max }}=\left\{\begin{array}{c}
X_{j}^{t, \text { max }}, \quad j \neq i \\
X_{i, \text { new }}^{t_{p} \text {,min }}+\left(X_{i, \text { new }}^{t_{p+1}, \text { min }}-X_{i, \text { new }}^{t_{p}, \min }\right)(1+\delta), \\
j=i, p<L_{i} \\
X_{i}^{t, \text { max }}, \quad j=i, p=L_{i} .
\end{array}\right.
$$

The detailed iterative decomposition process is described as follows.

Step 1) Calculate the division vector $\boldsymbol{L}$.

Step 2) Initialize $i=1$, which means we start the decomposition process from the first geometrical parameter $x_{1}$.

Step 3) Calculate the number of $i^{\text {th }}$ level sub-regions (i.e., $n$ ) based on Equation (5.21). 
The total number of the $(i-1)^{t h}$ level sub-regions is $n / L_{i}$.

Step 4) Set $t=1$. If $i=1$, there is only one region to be decomposed which is the original wide range $\boldsymbol{X}$, i.e., $\boldsymbol{X}^{t}=\boldsymbol{X}$.

Step 5) Count the number of training samples that lie in the range of $\boldsymbol{X}^{t}$, i.e., $N_{t}$.

Step 6) Reorder the $N_{t}$ training samples according to their values of $x_{i}$ in ascending order. The reordered training samples are $\hat{\boldsymbol{x}}^{k}$ where $k \in\left\{1,2, \cdots, N_{t}\right\}$.

Step 7) Calculate the total value of the second-order derivative w.r.t. $x_{i}$ at all $N_{t}$ training samples (i.e., $C_{i}^{t}$ ) based on Equation (5.24).

Step 8) Set $p=1$.

Step 9) Calculate $t_{p}=(t-1) L_{i}+p$.

Step 10) Calculate the range of $i^{\text {th }}$ level sub-region $t_{p}$ (i.e., $\boldsymbol{X}_{\text {new }}^{t_{p}}$ ) based on Equations (5.25) and (5.27).

Step 11) If $p<L_{i}$, update $p=p+1$ and go to Step 9). Otherwise, all the sub-ranges $\boldsymbol{X}_{\text {new }}^{t_{p}}$ for $p \in\left\{1,2, \cdots, L_{i}\right\}$ are obtained, the decomposition of $(i-1)^{t h}$ level sub-region $t$ is completed.

Step 12) If $t<n / L_{i}$, update $t=t+1$ and go to Step 5). Otherwise, the decomposition of $x_{i}$ is completed and all the $(i-1)^{t h}$ level sub-regions $\boldsymbol{X}^{t}$ where $t=1,2, \cdots, n / L_{i}$ are decomposed, we can obtain all the $i^{t h}$ level sub-regions $\boldsymbol{X}_{\text {new }}^{t_{p}}$ where $t_{p}=1,2, \cdots, n$. 
Step 13) Update $\boldsymbol{X}^{t_{p}}=\boldsymbol{X}_{\text {new }}^{t_{p}}$ for $t_{p}=1,2, \cdots, n$. We proceed to the decomposition of next variable $x_{i+1}$ by updating $i=i+1$.

Step 14) If $i<N$, go to Step 3). Otherwise, the last geometrical parameter $x_{N}$ is decomposed, the decomposition process is terminated and the total number of sub-regions is calculated as

$$
n=\prod_{j=1}^{N} L_{j}
$$

The flowchart of the proposed decomposition process is shown in Fig. 5.1. By using our proposed decomposition technique, the cumbersome decomposition process can be done systematically and automatically.

Let $\boldsymbol{\lambda}^{t}$ be a index vector of the $t^{t h}$ sub-model, defined as

$$
\boldsymbol{\lambda}^{t}=\left[\begin{array}{llll}
\lambda_{1}^{t} & \lambda_{2}^{t} & \cdots & \lambda_{N}^{t}
\end{array}\right]^{T}
$$

where the $\lambda_{i}^{t}$ represents the index of divisions at the $i^{t h}$ geometrical parameter for the sub-model $t$, i.e., $\lambda_{i}^{t} \in\left\{1,2, \cdots, L_{i}\right\}$. The vector $\boldsymbol{\lambda}^{t}$ has an one-to-one correspondence with the index number $t$, computed as

$$
\lambda_{i}^{t}=\left\{\begin{array}{l}
\left((t-1) \bmod L_{i}\right)+1, \quad i=N \\
\left(\left\lfloor(t-1) / \prod_{j=i+1}^{N} L_{j}\right\rfloor \bmod L_{i}\right)+1, i<N
\end{array}\right.
$$

where the symbol pair \lfloor\rfloor means the round off number and the operator mod represents the remainder number. 


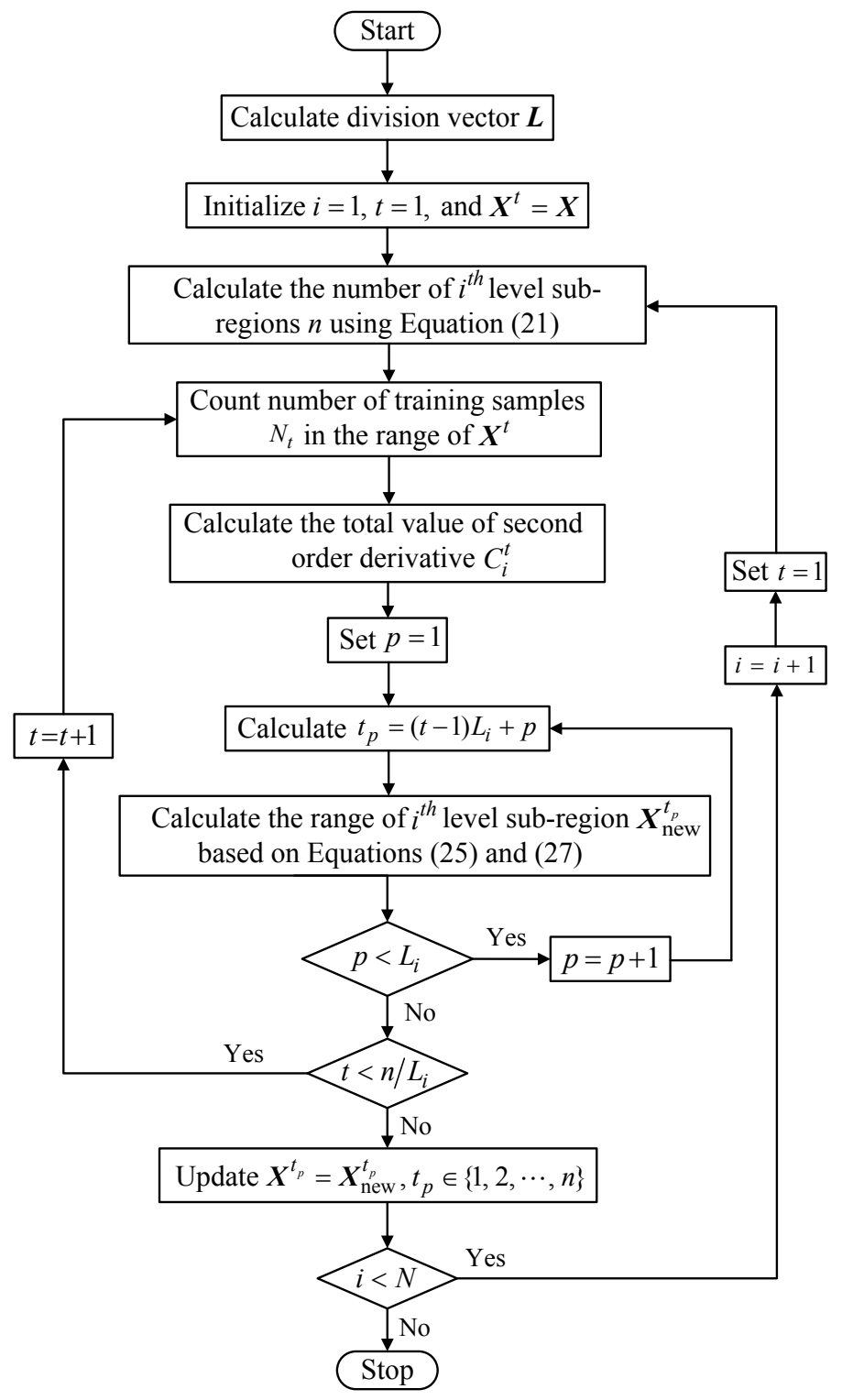

Figure 5.1: The proposed decomposition process to decompose the entire range $\boldsymbol{X}$ dimension by dimension for one training stage. The decomposition process starts from the first design variable $x_{1}$. When the last design variable $x_{N}$ is decomposed, we can automatically obtain the sub-ranges for all the sub-regions. 


\subsubsection{Parallel Sub-Model Training Process}

After the decomposition process is finished, we can obtain the sub-range of each decomposed sub-region. In our proposed technique, an artificial neural network model with simple structure is developed with geometrical parameters as variables in each sub-region. A parametric model (i.e., the ANN model) for a sub-region is called as a sub-model. A parametric model for the entire region is called as an overall model. The number of training samples and the ranges of geometrical parameters are different for different sub-models based on second order derivative information. Let $R_{s}^{t}(\boldsymbol{x}, f)$ represent the output of the $t^{t h}$ sub-model. Each sub-model has its own training range $\boldsymbol{X}^{t}$ and can be trained independently of other sub-models. Let $R_{f}^{t}(\boldsymbol{x}, f)$ represent the EM output response from the EM simulation in the range of $\boldsymbol{X}^{t}$. Training for the $t^{t h}$ sub-model is performed by optimizing the weights inside the neural networks to minimize the error function

$$
E^{t}=\frac{1}{2} \sum_{k=1}^{N_{t}} \sum_{q=1}^{N_{f}}\left\|R_{s}^{t}\left(\hat{\boldsymbol{x}}^{k}, \boldsymbol{w}_{t}, f_{q}\right)-R_{f}^{t}\left(\hat{\boldsymbol{x}}^{k}, f_{q}\right)\right\|^{2}
$$

where $\boldsymbol{w}_{t}$ represents the ANN internal weights of the $t^{t h}$ sub-model. Because the computation of error function $E^{t}$ is completely independent between different submodels, the formulation is naturally suitable for parallel training. In our proposed technique, parallel computational approaches are implemented to accelerate the sub-model training process.

The number of divisions $\boldsymbol{L}$ is updated stage by stage, which leads to an increase of the number of sub-models stage by stage. In order to make the sub-model training effective, the three-layer ANN structures with a small fixed number of hidden 
neurons are developed to represent the sub-regions for all the stages. After the sub-model training process, we can obtain the ANN sub-models within their own sub-ranges.

\subsection{Proposed Combination Technique for Wide Range Parametric Modeling}

In order to obtain the overall model which covers the entire wide geometrical range, we need to combine all the independently trained sub-models into one overall model. When the values of geometrical parameters change from the region of one sub-model to another sub-model, the discontinuity of EM responses is observed at the boundary between the adjacent sub-models. Given the specific value of the geometrical parameter which is located at the boundary between the adjacent sub-models, the responses of the adjacent sub-models are very similar but not necessarily equal. The responses are very similar because the adjacent sub-models are trained with same boundary data and are well trained in their own sub-range. The responses are not necessarily equal because the adjacent sub-models are separate sub-models and are trained separately. There are many sub-model boundaries in the overall model resulting in the complex multi-dimensional discontinuity problem. Thereby the overall model provided by directly combining all the trained sub-models cannot be used for design optimization. 


\subsubsection{The Discontinuity Problem for Adjacent Sub-models}

The discontinuity problem at the boundary between adjacent sub-models is a major issue for combining the trained sub-models to one overall continuous model. Here we illustrate the discontinuity problem using a fictitious two-dimensional (2D) example with four sub-ranges. In this $2 \mathrm{D}$ example with four sub-ranges, the design parameters are $\left[\begin{array}{ll}x_{1} & x_{2}\end{array}\right]$. The range of $x_{1}$ is from $X_{1}^{\min }=0$ to $X_{1}^{\max }=8$ and the range of $x_{2}$ is from $X_{2}^{\min }=0$ to $X_{2}^{\max }=8$. The total number of sub-models is $n=4$. The overall ranges are decomposed into the four sub-ranges which are $X^{1}=\left[\begin{array}{ll}0 & 4 \\ 0 & 4\end{array}\right]$, $X^{2}=\left[\begin{array}{ll}4 & 8 \\ 0 & 4\end{array}\right], X^{3}=\left[\begin{array}{ll}0 & 4 \\ 4 & 8\end{array}\right]$ and $X^{4}=\left[\begin{array}{ll}4 & 8 \\ 4 & 8\end{array}\right]$. After the sub-model training process, suppose we get the sub-models shown as

$$
R_{s}\left(x_{1}, x_{2}\right)=\left\{\begin{array}{l}
R_{s}^{1}\left(x_{1}, x_{2}\right)=x_{1}+x_{2}, x_{1} \in X^{1} \\
R_{s}^{2}\left(x_{1}, x_{2}\right)=1.1 x_{1}+1.2 x_{2}+0.8, x_{1} \in X^{2} \\
R_{s}^{3}\left(x_{1}, x_{2}\right)=1.2 x_{1}+1.3 x_{2}-0.3, x_{1} \in X^{3} \\
R_{s}^{4}\left(x_{1}, x_{2}\right)=1.4 x_{1}+1.4 x_{2}+0.5, x_{1} \in X^{4} .
\end{array}\right.
$$

Fig. 5.2 (a) shows the four sub-models after the training process. Fig. 5.2 (b) shows the sub-model responses along the diagonal line of $x_{1}$ and $x_{2}$ going through the sub-model 1 and sub-model 4 . From the figure, we can see that the outputs of the adjacent sub-models at the boundary are different and the overall model is not continuous.

In subsequent subsections, a parallel modification technique is proposed to mod- 


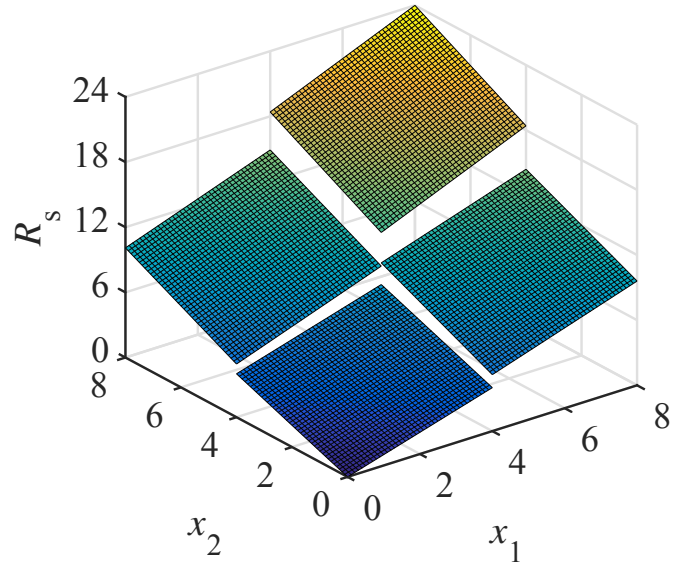

(a)

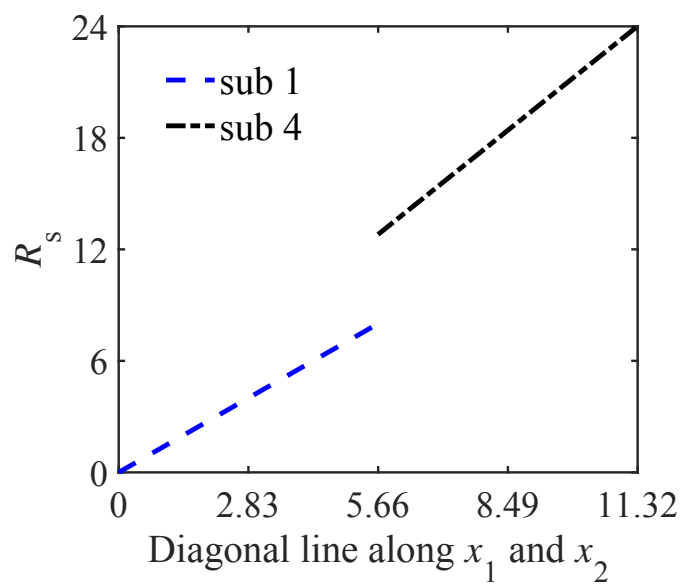

(b)

Figure 5.2: (a) The four sub-models after the training process for the 2D example with four sub-ranges. (b) The sub-model responses along the diagonal line of $x_{1}$ and $x_{2}$ going through the sub-model 1 and sub-model 4 . The outputs of the adjacent sub-models at the boundary are different and the overall model is not continuous.

ify the trained sub-models to solve the multi-dimensional discontinuity problem occurring at the boundary between the adjacent sub-models. After the proposed parallel modification process, we can combine all the sub-models and obtain an accurate and continuous model over the entire region.

\subsubsection{Proposed Technique to Solve the Discontinuity Prob- lem}

The overall model responses should not be represented by simply combining the trained sub-models due to the multi-dimensional discontinuity problem occurring at the boundary between adjacent sub-models. Here we first define the adjacent sub-models. Two independent sub-models $R_{s}^{t_{1}}(\boldsymbol{x}, f)$ and $R_{s}^{t_{2}}(\boldsymbol{x}, f)$ are adjacent sub- 
models when their index vector $\boldsymbol{\lambda}^{t_{1}}$ and $\boldsymbol{\lambda}^{t_{2}}$ satisfy the equation

$$
\max _{i \in\{1,2, \cdots, N\}}\left\|\lambda_{i}^{t_{1}}-\lambda_{i}^{t_{2}}\right\|=1, \quad t_{1} \neq t_{2}
$$

To solve the discontinuity problem occurring at the boundary between the adjacent sub-models, we propose a new algorithm to combine all sub-models to one overall wide range model. To achieve an accurate and continuous overall model, as described in Section 5.2.3, we propose to have an overlapping region for the adjacent sub-models along the dimension of each geometrical parameter. By using overlapping region, the same training data (i.e., data in the overlapping region) are independently used to train multiple adjacent sub-models at the boundary. After the sub-model training process, the adjacent sub-models have very similar output responses at the boundary. This can reduce the gap between the two adjacent submodels at the boundary. Furthermore, we propose to use the sigmoid function as the modification function to eliminate the remaining gap and solve the discontinuity problem completely. By using the proposed modification function, we can achieve the transition from one sub-model to another sub-model continuously. The standard sigmoid function is

$$
S(x)=\frac{1}{1+e^{-a(x-c)}}
$$

where the parameter $a$ can control the width of the transition range of the sigmoid function and the parameter $c$ is the center point of the transition. The demonstration of sigmoid functions with different values of $a$ and $c$ is shown in Fig. 5.3. We define the transition range of the sigmoid function to be the range where the output of the sigmoid function rises from $0.2 \%$ to $99.8 \%$. Let $W$ represent the transition 


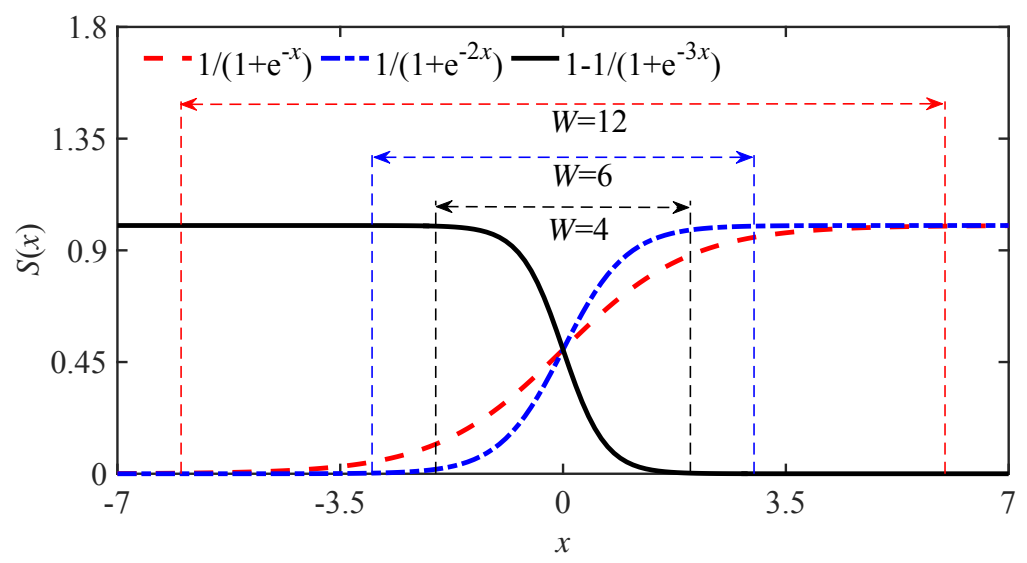

Figure 5.3: The demonstration of the sigmoid function with different values of $a$ and $c$. We can observe the relationship between the parameter $a$ and the transition range $W$.

range of the sigmoid function. From the figure, we can observe the relationship between the parameter $a$ and the transition range $W$, i.e.,

$$
W=\frac{12}{a} .
$$

The discontinuity problem occurs at the boundary between the adjacent sub-models for each dimension $x_{i}$. Therefore the purpose of our proposed technique is to modify the sub-model function at the boundary along each dimension, while keeping the sub-model function at the interior region unchanged. We exploit the sigmoid function to modify the trained sub-models along each dimension so that we can achieve the transition from one sub-model to another sub-model continuously. The sub-model has multiple design parameters leading to the multi-dimensional discontinuity problem. Many different sigmoid functions are needed to perform the modification process of the sub-model. 
For each sub-model, say $t^{\text {th }}$ sub-model, base on Equation (5.30), we can get the vector $\boldsymbol{\lambda}^{t}$ which has an one-to-one correspondence with the index number $t$. We modify the trained sub-model dimension by dimension based on the value of the index number $\lambda_{i}^{t}$ and division number $L_{i}$. If $L_{i}=1$, there is only one sub-model range along the direction of $x_{i}$, i.e., the original range. There is no discontinuity problem along the direction of $x_{i}$. Therefore there is no sigmoid function needed to perform the modification process to modify the responses along this dimension.

If $L_{i}>1$, there are more than one sub-model ranges along the direction of $x_{i}$. The entire range of $x_{i}$ has been decomposed into several divisions. If $\lambda_{i}^{t}=1$, it means the division $\lambda_{i}^{t}$ is the first division along the direction of $x_{i}$. The sub-model is modified at only one boundary which is the upper side of this division. We propose to use one sigmoid function to modify the sub-model at this boundary. Let $S_{i}^{t, u}$ represent the sigmoid function for modification at the upper boundary along the dimension of $x_{i}$, formulated as

$$
S_{i}^{t, u}(\boldsymbol{x})=\frac{1}{1+e^{-a_{i}^{t, u}\left(x_{i}-c_{i}^{t, u}\right)}}
$$

where the sigmoid function parameters $a_{i}^{t, u}$ and $c_{i}^{t, u}$ are calculated as

$$
\begin{aligned}
a_{i}^{t, u} & =\frac{12}{X_{i}^{t, \max }-X_{i}^{t_{u}, \min }} \\
c_{i}^{t, u} & =\frac{X_{i}^{t, \max }+X_{i}^{t_{u}, \min }}{2}
\end{aligned}
$$

where $t_{u}$ is the index number of the neighboring sub-model bordering at the upper side of the $t^{t h}$ sub-model along the direction of $x_{i}$. The detailed and complete 
calculation of the value of $t_{u}$ is provided in Appendix C. In this case, we modify the trained sub-model $R_{s}{ }^{t}$ by multiplying the coefficient $1-S_{i}^{t, u}$. Therefore, we can modify the sub-model function at the upper boundary along dimension $x_{i}$, while keeping the sub-model function at the interior region unchanged.

If $\lambda_{i}^{t}=L_{i}$, it means the division $\lambda_{i}^{t}$ is the last division along the direction of $x_{i}$. The sub-model is modified at only one boundary which is the lower side of this division. We propose to use one sigmoid function to modify the sub-model at this boundary. Let $S_{i}^{t, l}$ represent the sigmoid function for modification at the lower boundary along the dimension of $x_{i}$, formulated as

$$
S_{i}^{t, l}(\boldsymbol{x})=\frac{1}{1+e^{-a_{i}^{t, l}\left(x_{i}-c_{i}^{t, l}\right)}}
$$

where the sigmoid function parameters $a_{i}^{t, l}$ and $c_{i}^{t, l}$ are calculated as

$$
\begin{aligned}
a_{i}^{t, l} & =\frac{12}{X_{i}^{t_{l}, \max }-X_{i}^{t, \min }} \\
c_{i}^{t, l} & =\frac{X_{i}^{t_{l}, \max }+X_{i}^{t, \min }}{2}
\end{aligned}
$$

where $t_{l}$ is the index of the neighboring sub-model bordering at the lower side of the $t^{t h}$ sub-model along the direction of $x_{i}$. The detailed and complete calculation of the value of $t_{l}$ is provided in Appendix D. In this case, we modify the trained sub-model $R_{s}{ }^{t}$ by multiplying the coefficient $S_{i}^{t, l}$. Therefore, we can modify the sub-model function at the lower boundary along dimension $x_{i}$, while keeping the sub-model function at the interior region unchanged. Otherwise, if $1<\lambda_{i}^{t}<L_{i}$, the division $\lambda_{i}^{t}$ is located in a middle division along the direction of $x_{i}$. The sub-model 
is modified at both boundaries which are the lower and upper sides of this division. Both $S_{i}^{t, l}$ and $S_{i}^{t, u}$ are needed to modify the sub-model.

The modification function for the $t^{t h}$ sub-model along the direction of $x_{i}$ is formulated as

$$
\Psi_{i}^{t}(\boldsymbol{x})=\left\{\begin{array}{l}
1, \quad L_{i}=1 \\
\left(1-S_{i}^{t, u}\right), \quad L_{i}>1, \lambda_{i}^{t}=1 \\
S_{i}^{t, l} \cdot\left(1-S_{i}^{t, u}\right), \quad L_{i}>1,1<\lambda_{i}^{t}<L_{i} \\
S_{i}^{t, l}, \quad L_{i}>1, \lambda_{i}^{t}=L_{i}
\end{array}\right.
$$

where the symbol $\Psi_{i}^{t}(\boldsymbol{x})$ represents the modification function along the direction of $x_{i}, i=1,2, \cdots, N$. The modification process is performed dimension by dimension. After going through all dimensions, we can get the total modification function for the $t^{\text {th }}$ sub-model, formulated as

$$
\Psi^{t}(\boldsymbol{x})=\prod_{i=1}^{N} \Psi_{i}^{t}(\boldsymbol{x})
$$

The flowchart for calculating the total modification function for the $t^{\text {th }}$ sub-model is shown in Fig. 5.4. It is noticed that for different sub-models, the number of the sigmoid functions in the total modification function can be different. The revised sub-model function after modification is formulated as

$$
R_{s}^{t, \text { new }}(\boldsymbol{x})=R_{s}^{t}(\boldsymbol{x}) \cdot \Psi^{t}(\boldsymbol{x}) .
$$

The modification process is completely independent for different sub-models. The formulation is naturally suitable for parallel processing. In our proposed tech- 


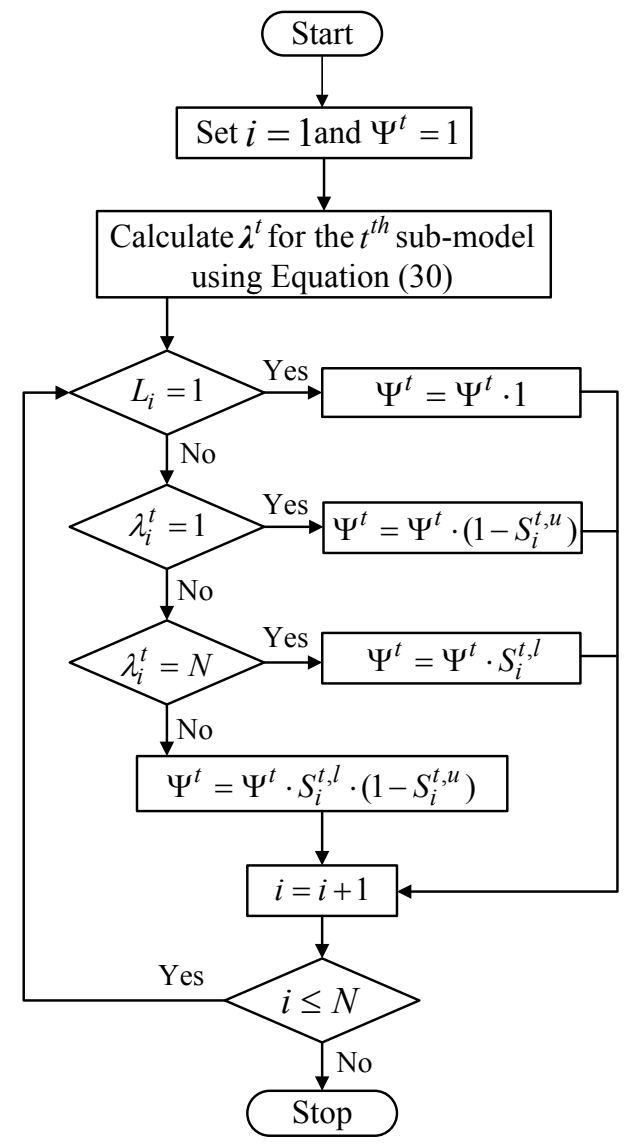

Figure 5.4: The flowchart for calculating the total modification function of $\Psi^{t}$ for the $t^{\text {th }}$ sub-model.

nique, parallel modification process is implemented for different sub-models to accelerate the overall training process.

After we perform the modification process for all the sub-models, the last step is to combine all the modified sub-models together to get the overall model which covers the entire geometrical range. The overall model is formulated as

$$
R_{s}(\boldsymbol{x})=\sum_{t=1}^{n} R_{s}^{t, \text { new }}(\boldsymbol{x}) .
$$


By controlling the transition range of the sigmoid function using Equation (5.35), we can keep the sub-model function $R_{s}^{t}$ at the interior region unchanged while modify the sub-model only at the boundary. In this way, we achieve the transition from one sub-model to another sub-model continuously and at the same time guarantee the accuracy of the overall model.

This completes one training stage which includes performing the decomposition for all the geometrical parameters, training the decomposed sub-models using parallel training, and combining the developed sub-models into overall model. Once we obtain the overall model, we calculate the overall model training and testing error using the entire training and testing data. With the given limited number of hidden neurons, if the testing error is lower than a user-defined threshold $\varepsilon$, the training process terminates and the overall wide range model has been developed. Otherwise, we set $K=K+1$ and update the number of division vector $\boldsymbol{L}$ (i.e., increase number of sub-models) using Equation (5.20) for the next training stage. The flowchart of the iterative development process of the wide range parametric model using decomposition technique is illustrated in Fig. 5.5. The proposed technique takes several training stages to obtain an accurate and continuous overall model. Once the overall wide range model is developed, it is ready to be used for higher level design optimization.

To further explain our proposed modification process to solve the discontinuity problem between the adjacent sub-models, we illustrate the idea using the $2 \mathrm{D}$ example with four sub-ranges in Section 3.1. For the 2D fictitious example, using our proposed technique, the sigmoid function is exploited to modify the sub-models. 
There are four sub-models in this example, i.e., $R_{s}^{1}, R_{s}^{2}, R_{s}^{3}$, and $R_{s}^{4}$. For $R_{s}^{1}$, the sub-model is modified at the upper side boundary along $x_{1}$ and also at the upper side boundary along $x_{2}$. Based on our proposed technique, the modification function $\Psi_{1}^{1}$ for sub-model 1 along the direction of $x_{1}$ is $\left(1-S_{1}^{1, u}\left(x_{1}, x_{2}\right)\right)$ and the modification function $\Psi_{2}^{1}$ for sub-model 1 along the direction of $x_{2}$ is $\left(1-S_{2}^{1, u}\left(x_{1}, x_{2}\right)\right)$. Similarly, we can formulate the modification function for other sub-models using the proposed technique. We modify all the sub-models and combine all the modified sub-models together to obtain the overall model for this $2 \mathrm{D}$ problem. The overall model function is formulated as

$$
\begin{aligned}
& R_{s}\left(x_{1}, x_{2}\right)=R_{s}^{1} \cdot \Psi^{1}+R_{s}^{2} \cdot \Psi^{2}+R_{s}^{3} \cdot \Psi^{3}+R_{s}^{4} \cdot \Psi^{4} \\
& \quad=R_{s}^{1}\left(x_{1}, x_{2}\right) \cdot\left(1-S_{1}^{1, u}\left(x_{1}, x_{2}\right)\right) \cdot\left(1-S_{2}^{1, u}\left(x_{1}, x_{2}\right)\right) \\
& \quad+R_{s}^{2}\left(x_{1}, x_{2}\right) \cdot\left(1-S_{1}^{2, u}\left(x_{1}, x_{2}\right)\right) \cdot S_{2}^{2, l}\left(x_{1}, x_{2}\right) \\
& \quad+R_{s}^{3}\left(x_{1}, x_{2}\right) \cdot S_{1}^{3, l}\left(x_{1}, x_{2}\right) \cdot\left(1-S_{2}^{3, u}\left(x_{1}, x_{2}\right)\right) \\
& \quad+R_{s}^{4}\left(x_{1}, x_{2}\right) \cdot S_{1}^{4, l}\left(x_{1}, x_{2}\right) \cdot S_{2}^{4, l}\left(x_{1}, x_{2}\right) .
\end{aligned}
$$

From the above overall model equation, the modification function for each submodel can be observed clearly. Fig. 5.6 (a) demonstrates the overall model function after the proposed modification process for this 2D example. Fig. 5.6 (b) shows the connecting result along the diagonal line going through the sub-model 1 and sub-model 4. From the figure, we can see that after the modification process, the continuous model over the entire region is obtained. Our proposed technique can successfully solve the discontinuity problem. 


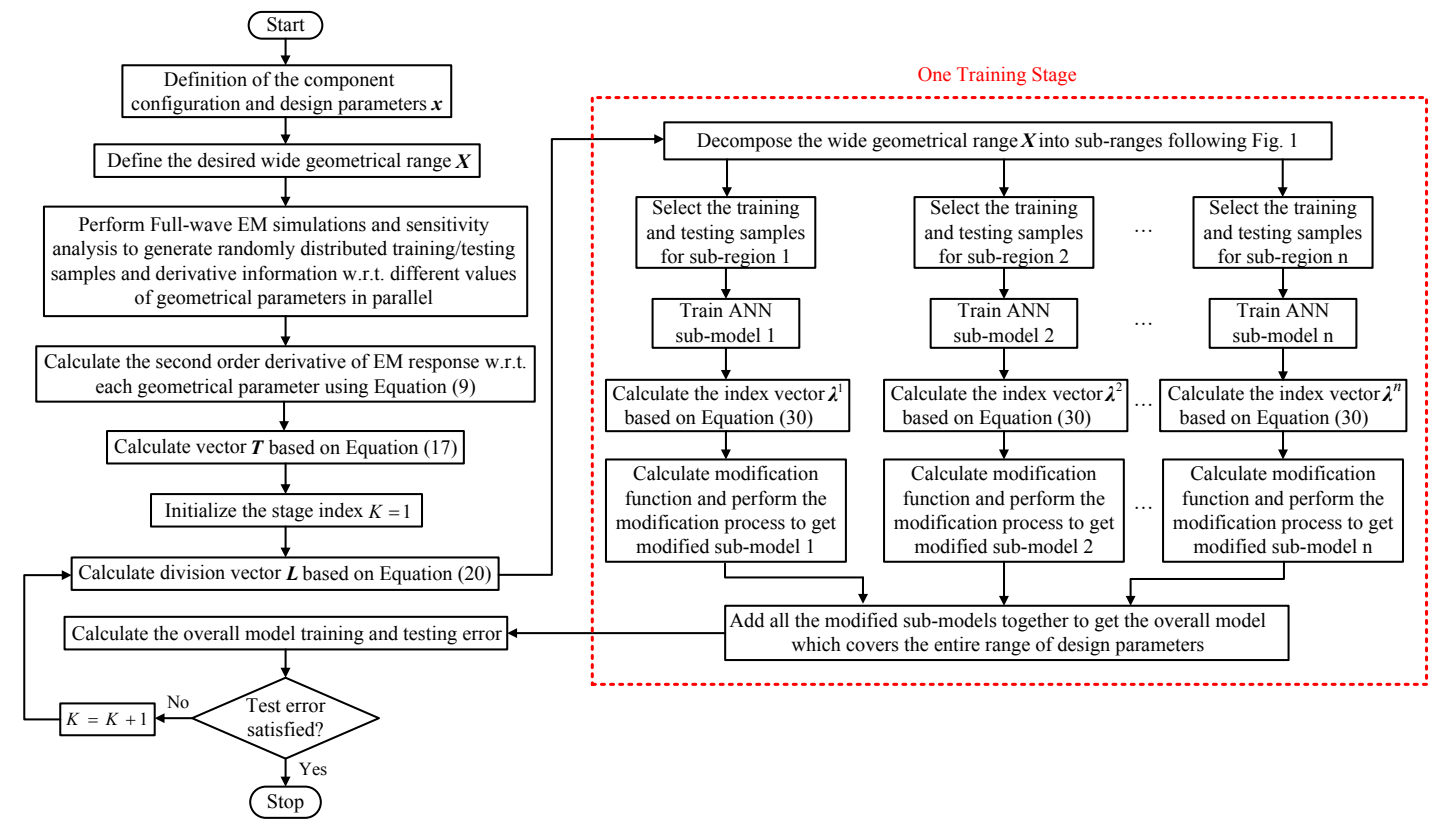

Figure 5.5: The flowchart for developing the proposed parametric model using decomposition technique. The proposed technique takes several training stages to obtain an accurate and continuous overall model. Each training stage includes performing the decomposition for all the geometrical parameters, training the ANN sub-models using parallel training, and combining the developed sub-models into overall model.

\subsection{Numerical Examples}

\subsubsection{Parametric Modeling of a Bandstop Microstrip Filter with Open Stubs Incorporating Decomposition Tech- nique}

In order to illustrate the validity of the proposed decomposition technique, we consider a bandstop microstrip filter with quarter-wave resonant open stubs [92]. The structure of this bandstop microstrip filter is shown in Fig. 5.7. For this example, length $l_{0}$ is the interconnecting transmission line length between the two open stubs. 


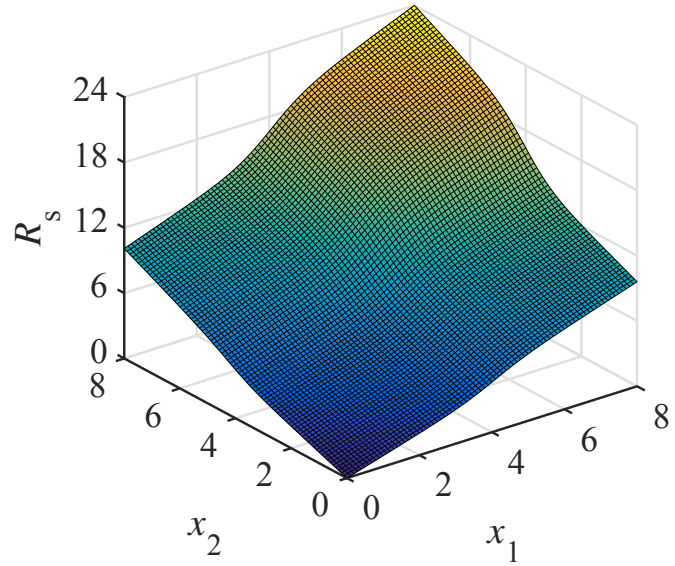

(a)

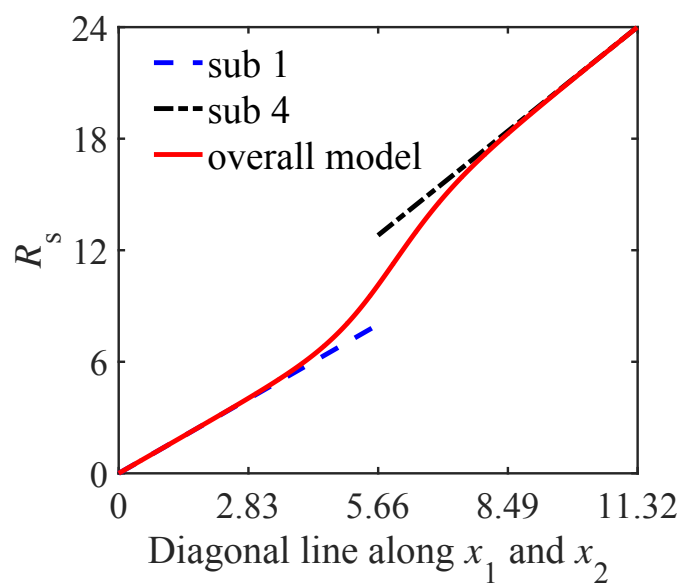

(b)

Figure 5.6: (a) The overall model function after the proposed modification process for the 2D example with four sub-ranges. (b) The connection result along the diagonal line of $x_{1}$ and $x_{2}$ going through the sub-model 1 and sub-model 4 . After the modification process, a continuous model over the entire region is obtained. Our proposed technique can successfully solve the discontinuity problem.

Lengths $l_{1}$ and $l_{2}$ are the open stub lengths. An alumina substrate with thickness of $0.635 \mathrm{~mm}$ and dielectric constant 9.4 is used along with a $50 \Omega$ feeding line of width $0.635 \mathrm{~mm}$. Widths of the microstrip open stubs are set as $w_{1}=0.10523 \mathrm{~mm}$ and $w_{2}=0.2097 \mathrm{~mm}$. The design variables for this example are $\boldsymbol{x}=\left[\begin{array}{lll}l_{0} & l_{1} & l_{2}\end{array}\right]$. Frequency $f$ is an additional input. The model has two outputs, i.e., $R S_{11}$ and $I S_{11}$ which are the real and imaginary parts of the overall model output $S_{11}$ w.r.t. different values of geometrical input parameters.

For this example, the frequency range is from $5 \mathrm{GHz}$ to $20 \mathrm{GHz}$. The number of frequency points is 151 . The range of the design variable $l_{0}$ is from $2 \mathrm{~mm}$ to 5.2 $\mathrm{mm}$, the range of the design variable $l_{1}$ is from $2.2 \mathrm{~mm}$ to $5.4 \mathrm{~mm}$, and the range 


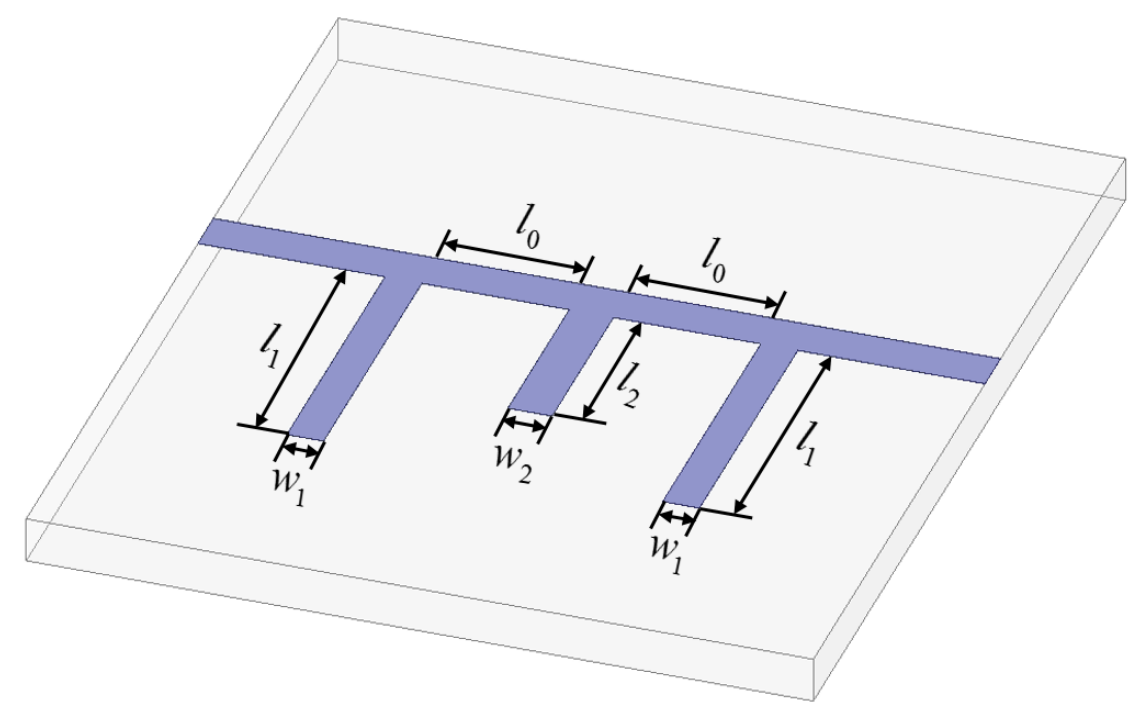

Figure 5.7: The bandstop microstrip filter with quarter-wave resonant open stubs. The design variables for this example are $\boldsymbol{x}=\left[l_{0} l_{1} l_{2}\right]$.

of the design variable $l_{2}$ is from $1.8 \mathrm{~mm}$ to $5 \mathrm{~mm}$. The geometrical range for this example is

$$
\boldsymbol{X}=\left[\begin{array}{ll}
2 & 5.2 \\
2.2 & 5.4 \\
1.8 & 5
\end{array}\right]
$$

Using a single neural network model is not enough to represent the behavior of the wide range responses. To build an accurate overall model, we use randomly distributed data generation method to generate the training and testing data. For this example, the number of training sample is 5000. The EM evaluation is performed by HFSS EM simulator. First order EM derivatives are obtained directly from the HFSS simulator. We use a cluster of Dell PowerEdge computers with Intel Xeon X5680 processor with each computer having eight processing cores. Us- 
ing this cluster, the training samples are generated in parallel. After the parallel data generation, to decompose the entire geometrical ranges using our proposed decomposition technique, we first evaluated the second-order EM derivative for each geometrical parameter. Based on the calculation method described in Section 5.2.2, the number of unknown elements $N_{u}$ in the Hessian matrix is 6 . To get good model accuracy, we use 12 neighboring samples (i.e., $N_{h}=12$ ) to calculate coefficients of Hessian matrix. After solving the Equation (5.9), we can get the second-order derivative of EM response w.r.t. three geometrical variables for each training data at each frequency point. The average values of second-order derivative are calculate as $\left[\begin{array}{lll}Q_{1} & Q_{2} & Q_{3}\end{array}\right]=\left[\begin{array}{lll}205.64 & 261.9 & 244.65\end{array}\right]^{T}$.

Once we obtain the second-order derivatives, we can decompose the overall geometrical range into multiple sub-ranges. For the first training stage, i.e., $K=1$, the number of division $\boldsymbol{L}$ is calculated using Equation (5.20) as $\left[\begin{array}{lll}1 & 1 & 1\end{array}\right]$. This means that for the first training stage, there is only one sub-model which is the overall model. To train the sub-model efficiently, in this example, a three-layer ANN with 50 hidden neurons is used to train the sub-models for all the training stages. The training and testing error for the first training stage is $7.27 \%$ and $7.28 \%$, respectively. The user-defined threshold for the testing error is below $2 \%$. The overall model after first training stage cannot satisfy the required error.

For the second training stage, i.e., $K=2$, the number of division $\boldsymbol{L}$ is calculated as $\left[\begin{array}{lll}2 & 3 & 2\end{array}\right]$. The total number of sub models is $n=12$ for the second training stage. Using the second-order derivative information, we can obtain the specific sub-range for each sub-model using the proposed decomposition process in Fig. 5.1. In this 


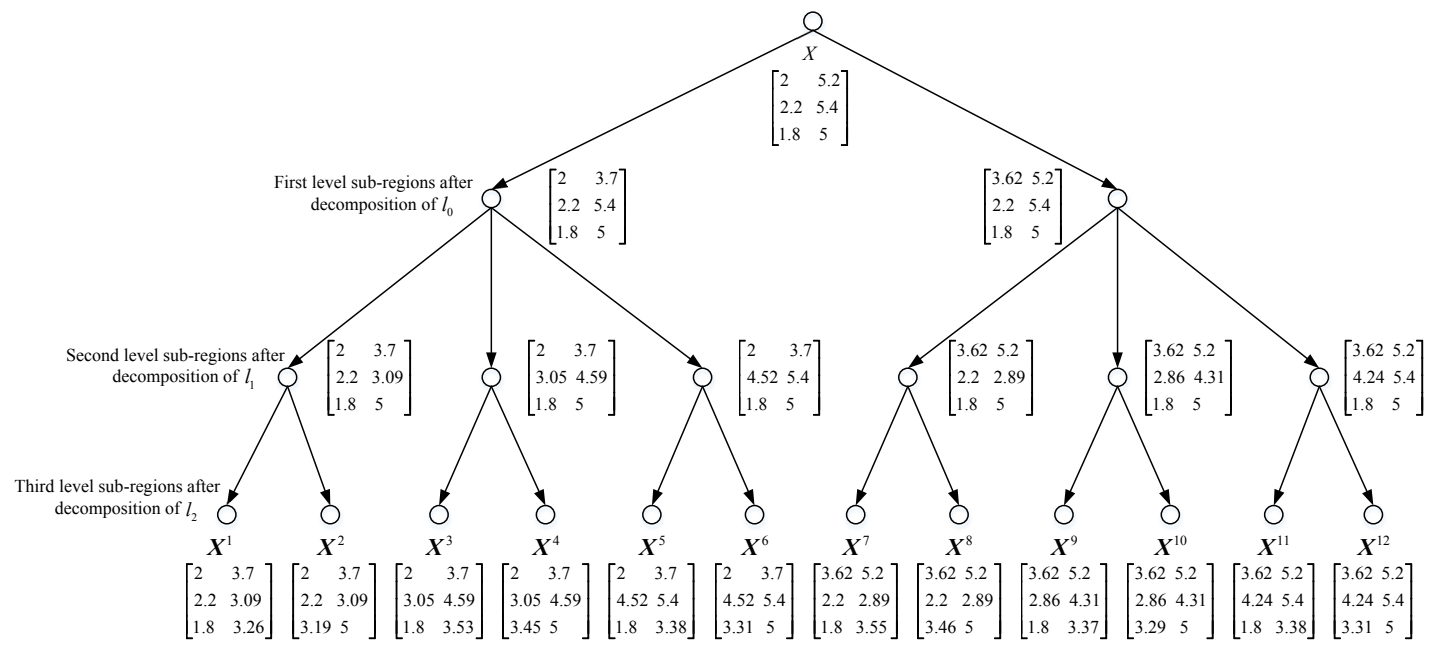

Figure 5.8: The sub-range for each sub-model after decomposition process at training stage two for the bandstop microstrip filter example. The design parameters for this example are $\boldsymbol{x}=\left[l_{0} l_{1} l_{2}\right]$. At the second training stage, the number of division $\boldsymbol{L}$ is calculated as $\left[\begin{array}{lll}2 & 3 & 2\end{array}\right]$. At the first level decomposition, the range of geometrical parameter $l_{0}$ is decomposed into 2 sub-ranges. At the second level decomposition, the range of geometrical parameter $l_{1}$ is decomposed into 3 sub-ranges. At the third level decomposition, the range geometrical parameter $l_{2}$ is decomposed into 2 sub-ranges. The total number of sub-models is $n=2 \cdot 3 \cdot 2=12$.

example, we define the overlapping coefficient $\delta=5 \%$. The sub-range for each geometrical parameter after decomposition process is shown in Fig. 5.8.

Based on the sub-model range shown in Fig. 5.8, we can select the training and testing data for each sub-model from the overall training and testing data sets. We can train the sub-models using the parallel training technique to accelerate the training process. After the parallel training process is finished, we combine all the independently trained sub-models to obtain the overall model which covers the entire wide geometrical range. We should not simply combine all the sub-models because 
of the discontinuity problem at the boundary between adjacent sub-models. To solve the discontinuity problem so that we can obtain the continuous overall model over the entire range, the proposed parallel modification process is performed for each trained sub-model following the flowchart in Fig. 5.4. For the sub-model $t$ where $t=1,2, \cdots, 12$, the modification function along the direction of $x_{i}$ is calculated using Equation (5.42).

After we perform the modification process for all the sub-models, the last step is to add all the modified sub-models together to get the overall model which covers the entire geometrical parameter range. We use all the training and testing data to test the overall model. The average training error for the overall model is $2.93 \%$, while the average testing error is $2.96 \%$. The user-defined threshold for the testing error is below $2 \%$. The overall model after the second training stage still cannot satisfy the required error.

For the third training stage, i.e., $K=3$, the number of division $\boldsymbol{L}$ is calculated as [3 4 4]. The total number of sub models is $n=48$ for the third training stage. Using the second-order derivative information, we can obtain the specific sub-range for each sub-model using the proposed decomposition process in Fig. 5.1. Based on the sub-range for each sub-model, we can select the training and testing data for each sub-model from the overall training and testing data sets. After the parallel training process is finished, we combine all the independently trained sub-models into one overall model to obtain the overall model. To solve the discontinuity problem to obtain the continuous overall model over the entire range, the proposed parallel modification process is performed for each trained sub-model following the 
flowchart in Fig. 5.4.

After we perform the modification process for all the sub-models, the last step is to add all the modified sub-models together to get the overall model which covers the entire geometrical parameter range. We use all the training and testing data to test the overall model. The average training error for the overall model is $1.76 \%$, while the average testing error is $1.78 \%$. The overall model after third training stage satisfies the required error threshold. After third training stage, using our proposed decomposition technique, we build the accurate and continuous wide range parametric model which consists of 48 sub-models.

For comparison purpose, we also use the standard modeling methods using a single model to cover the entire wide geometrical range. A single ANN model is directly trained to learn the entire range of the geometrical parameters for five cases: case 1 being with 50 hidden neurons, case 2 being with 100 hidden neurons, case 3 being with 200 hidden neurons, case 4 being with 400 hidden neurons, and case 5 being with 1000 hidden neurons. Table 5.1 compares the different parametric modeling methods in terms of ANN structures, average training and testing error, and CPU time. From the table, we can see that our proposed model combining many sub-models is more accurate than the standard ANN model using a single ANN for the entire wide range modeling. The reason is that the proposed technique focuses on learning each small sub-region by a separate sub-model, while the standard ANN technique using a single ANN for the entire wide range modeling needs to compromise the ANN accuracy between different regions. Also our proposed technique uses less CPU time to achieve the accurate overall model since we incorporate the 
Table 5.1: Comparisons of Different Methods for Parametric Modeling of the Bandstop Filter Example

\begin{tabular}{|c|c|c|c|c|}
\hline Modeling Method & $\begin{array}{l}\text { No. of Hidden } \\
\text { Neurons }\end{array}$ & $\begin{array}{l}\text { Average } \\
\text { Training Error }\end{array}$ & $\begin{array}{c}\text { Average } \\
\text { Testing Error }\end{array}$ & $\begin{array}{l}\text { CPU } \\
\text { Time }\end{array}$ \\
\hline ANN Model ${ }^{+}$ & 50 & $7.27 \%$ & $7.28 \%$ & $3.15 h$ \\
\hline ANN Model + & 100 & $5.81 \%$ & $5.80 \%$ & $6.69 h$ \\
\hline ANN Model ${ }^{+}$ & 200 & $4.11 \%$ & $4.12 \%$ & $12.9 h$ \\
\hline ANN Model ${ }^{+}$ & 400 & $3.77 \%$ & $3.79 \%$ & $26.4 h$ \\
\hline ANN Model ${ }^{+}$ & 1000 & $3.75 \%$ & $3.76 \%$ & $65 h$ \\
\hline $\begin{array}{c}\text { Proposed Model at Training } \\
\text { Stage } 1 \text { Without Parallel }\end{array}$ & $\begin{array}{c}50 \\
1 \text { sub-model }\end{array}$ & $7.27 \%$ & $7.28 \%$ & $3.15 h$ \\
\hline $\begin{array}{c}\text { Proposed Model at Training } \\
\text { Stage } 2 \text { Without Parallel }\end{array}$ & $\begin{array}{c}50 \\
12 \text { sub-models }\end{array}$ & $2.93 \%$ & $2.96 \%$ & $3.3 h$ \\
\hline $\begin{array}{c}\text { Proposed Model at Training } \\
\text { Stage } 3 \text { Without Parallel }\end{array}$ & $\begin{array}{c}50 \\
48 \text { sub-models }\end{array}$ & $1.76 \%$ & $1.78 \%$ & $3.48 h$ \\
\hline $\begin{array}{l}\text { Final Proposed Model } \\
\text { Without Parallel }\end{array}$ & $\begin{array}{c}50 \\
48 \text { sub-models }\end{array}$ & $1.76 \%$ & $1.78 \%$ & $9.93 h$ \\
\hline $\begin{array}{l}\text { Proposed Model at Training } \\
\text { Stage } 1 \text { With Parallel }\end{array}$ & $\begin{array}{c}50 \\
1 \text { sub-model }\end{array}$ & $7.27 \%$ & $7.28 \%$ & $3.15 h$ \\
\hline $\begin{array}{l}\text { Proposed Model at Training } \\
\text { Stage } 2 \text { With Parallel }\end{array}$ & $\begin{array}{c}50 \\
12 \text { sub-models }\end{array}$ & $2.93 \%$ & $2.96 \%$ & $24.2 \mathrm{~min}$ \\
\hline $\begin{array}{c}\text { Proposed Model at Training } \\
\text { Stage } 3 \text { With Parallel }\end{array}$ & $\begin{array}{c}50 \\
48 \text { sub-models }\end{array}$ & $1.76 \%$ & $1.78 \%$ & $6.1 \mathrm{~min}$ \\
\hline $\begin{array}{l}\text { Final Proposed Model } \\
\text { With Parallel }\end{array}$ & $\begin{array}{c}50 \\
48 \text { sub-models }\end{array}$ & $1.76 \%$ & $1.78 \%$ & $3.66 h$ \\
\hline
\end{tabular}

+ Standard ANN model using a single ANN for the entire wide

range modeling

* Final result of proposed model without parallel after 3 stages of training

$\dagger$ Final result of proposed model with parallel after 3 stages of training

parallel training technique.

The comparison of the magnitude of $S_{11}$ between three models (the proposed model, ANN model with 50 hidden neurons, and ANN model with 1000 hidden neurons) for two different filter test geometries are shown in Fig. 5.9. The two test 
samples are from testing data and have never been used in training process. The values of the two test samples are as follows

Test sample \#1: $\boldsymbol{x}=\left[\begin{array}{lll}5.15 & 4.95 & 2.765\end{array}\right]^{T} \mathrm{~mm}$

Test sample \#2: $\boldsymbol{x}=\left[\begin{array}{lll}3.405 & 4.075 & 2.985\end{array}\right]^{T} \mathrm{~mm}$

We use several graphs to demonstrate the continuity of the proposed overall model. Fig. 5.10 (a) shows the real and imaginary parts of $S_{11}$ along the direction of $l_{2}$. The values of the other parameters are fixed as $l_{0}=4.2 \mathrm{~mm}, l_{1}=2.6 \mathrm{~mm}$, and $f=8 \mathrm{GHz}$. Fig. 5.10 (b) shows the real and imaginary parts of $S_{11}$ along the diagonal direction of $l_{1}$ and $l_{2}$. The values of the other parameters are fixed as $l_{0}=4.2 \mathrm{~mm}$ and $f=8 \mathrm{GHz}$. Fig. 5.10 (c) shows the real and imaginary parts of $S_{11}$ along the diagonal direction of $l_{0}, l_{1}$, and $l_{2}$ at $16 \mathrm{GHz}$. From the figure, we can see that our proposed technique provides accurate and also continuous solutions compared to the HFSS simulation results across the boundaries along various directions.

Since the proposed wide range parametric model is accurate and continuous, we can implement the trained model into the design optimization where the design parameters can be repetitively adjusted during optimization. We perform the design optimization for the bandstop microstrip filter with the design specifications:

$$
\begin{aligned}
& \left|S_{11}\right| \leq 0.4, \quad 7.2 \mathrm{GHz} \leq f \leq 10.2 \mathrm{GHz} \\
& \left|S_{11}\right| \geq 0.94, \quad 11.2 \mathrm{GHz} \leq f \leq 13.2 \mathrm{GHz} \\
& \left|S_{11}\right| \leq 0.4, \quad 14.2 \mathrm{GHz} \leq f \leq 17.2 \mathrm{GHz}
\end{aligned}
$$

The design variables are the model inputs $\boldsymbol{x}=\left[l_{0} l_{1} l_{2}\right]$. The initial values are 


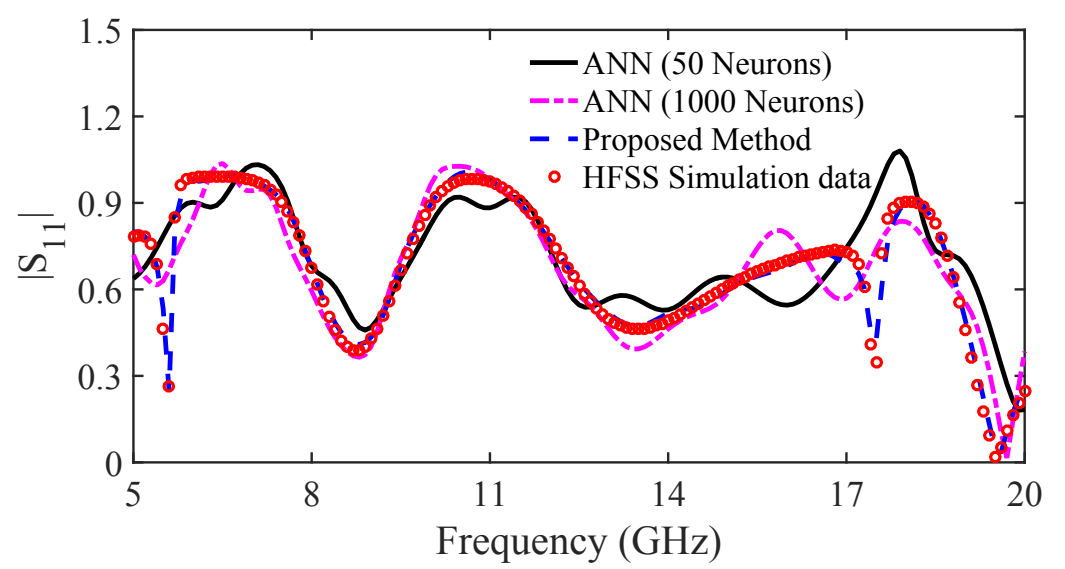

(a)

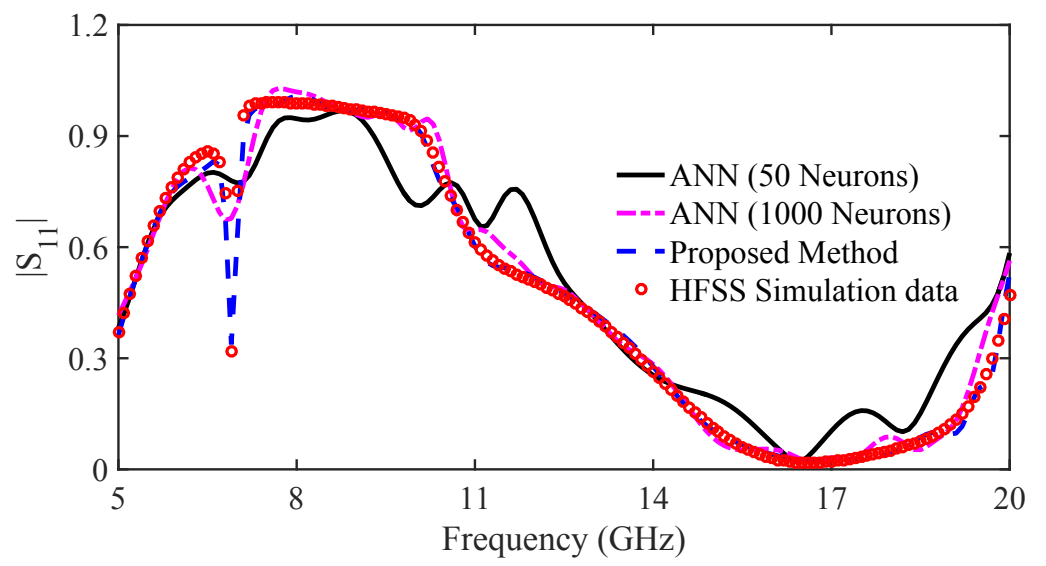

(b)

Figure 5.9: Comparison of the magnitude of $S_{11}$ of the overall parametric model developed using different modeling methods and HFSS simulation data: (a) test sample \#1 and (b) test sample \#2 for the bandstop microstrip example. Our proposed model combining many sub-models is more accurate than standard ANN model using a single ANN for the entire wide range modeling because the proposed technique focuses on learning each small sub-region by a separate sub-model, while the standard modeling technique using a single ANN for the entire wide range modeling needs to compromise the ANN accuracy between different regions. 


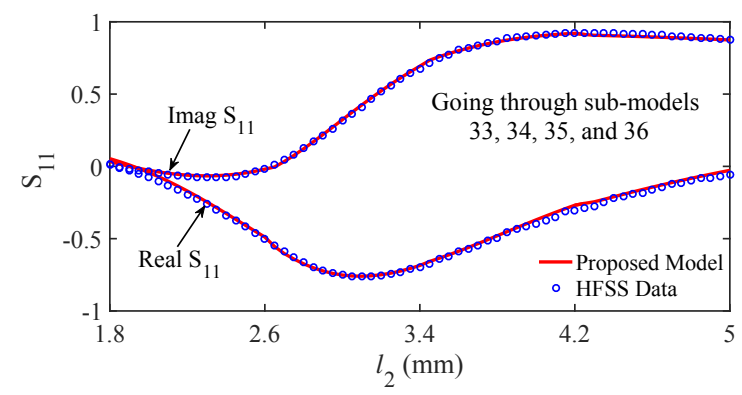

(a)

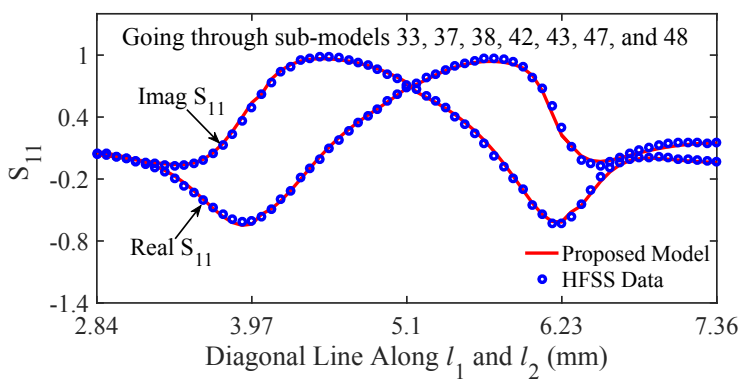

(b)

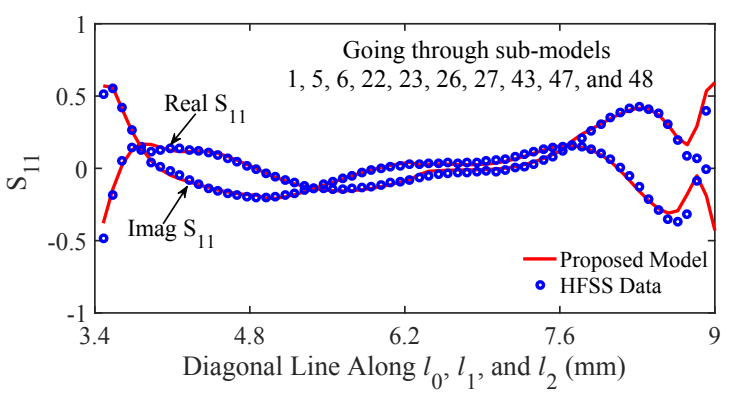

(c)

Figure 5.10: Demonstration of the continuity of the proposed overall model with design variables $\left[l_{0} l_{1} l_{2}\right]$. The real and imaginary parts of $S_{11}$ (a) along the direction of $l_{2}$ going through sub-models 33, 34, 35, and 36; (b) along the diagonal direction of $l_{1}$ and $l_{2}$ going through sub-models $33,37,38,42,43,47$, and 48; and (c) along the diagonal direction of $l_{0}, l_{1}$, and $l_{2}$ going through sub-models $1,5,6,22,23,26$, $27,43,47$, and 48 . The proposed technique provides accurate and also continuous solutions along different directions. Our solutions are verified with HFSS simulation results. 
$\boldsymbol{x}=\left[\begin{array}{lll}3.8 & 4.8 & 3.6\end{array}\right]^{T} \mathrm{~mm}$. This initial point actually lies in the sub-range of sub-model 23. The design optimization using the proposed overall model takes only about 20 seconds to achieve the optimal design solution for the specification. The optimized design parameter values for the bandstop microstrip cavity filter are

$$
\boldsymbol{x}=\left[\begin{array}{lll}
2.61103 & 2.31636 & 2.20772
\end{array}\right]^{T} \mathrm{~mm} .
$$

This optimized solution point lies in the sub-range of the sub-model 1 instead of sub-model 23. The HFSS simulations at the initial point and the optimal solution are shown in Fig. 5.11. Our proposed model behaves well in design optimization where the optimization moves freely and continuously between different sub-models.

\subsubsection{Parametric Modeling of an Inter-Digital Bandpass Filter Incorporating Decomposition Technique}

We consider a standard inter-digital bandpass filter [32], which is shown in Fig. 5.12. Assume equal spacing $(g)$ between each end of the resonator and the cavity wall. Coupling ratio between resonators are adjusted by tuning the values of spacing $\left(s_{1}\right)$, spacing $\left(s_{2}\right)$, and spacing $\left(s_{3}\right)$ between resonators. Each resonator is of length 43.18 $\mathrm{mm}$, width $5 \mathrm{~mm}$, thickness $0.5 \mathrm{~mm}$, and the structure is enclosed in an cavity of height $10 \mathrm{~mm}$. The design variables for this example are $\boldsymbol{x}=\left[\begin{array}{llll}g & s_{1} & s_{2} & s_{3}\end{array}\right]$. Frequency $f$ is an additional input. The model has two outputs, i.e., $R S_{11}$ and $I S_{11}$ which are the real and imaginary parts of the overall model output $S_{11}$ w.r.t. different values of geometrical input parameters.

For this example, the frequency range is from $0.6 \mathrm{GHz}$ to $2.4 \mathrm{GHz}$. The number of frequency points is 181 . The range of the design variable $g$ is from $2.2 \mathrm{~mm}$ to 5.8 


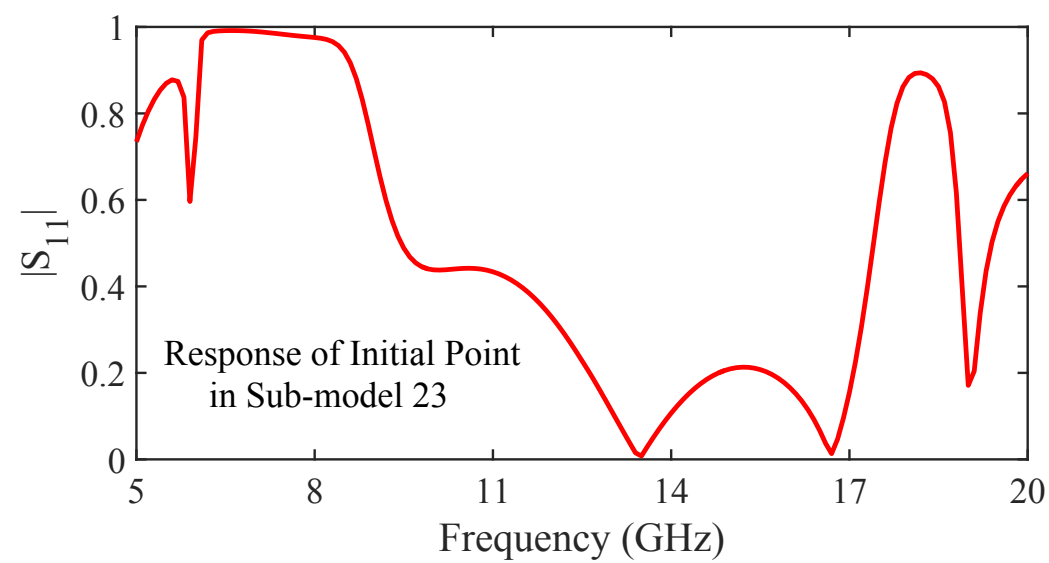

(a)

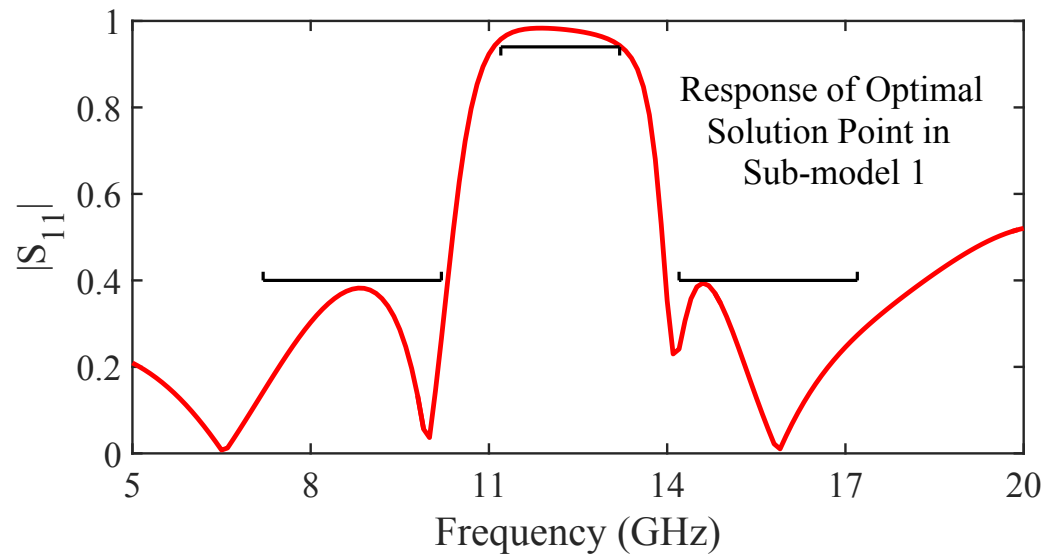

(b)

Figure 5.11: The proposed parametric model is used for design optimization for the bandstop microstrip filter. The optimal solution is found by optimizing design variables $\left[\begin{array}{lll}l_{0} & l_{1} & l_{2}\end{array}\right]$ using our proposed model. The optimal solution is verified by HFSS simulation. The magnitude of $S_{11}$ of HFSS simulation data at (a) initial point in sub-model 23 and (b) optimal solution point in sub-model 1. Our proposed model behaves well in design optimization where the optimization moves freely and continuously between different sub-models.

$\mathrm{mm}$, the range of the design variable $s_{1}$ is from $0.5 \mathrm{~mm}$ to $2.9 \mathrm{~mm}$, the range of the design variable $s_{2}$ is from $0.6 \mathrm{~mm}$ to $3 \mathrm{~mm}$, and the range of the design variable $s_{3}$ 


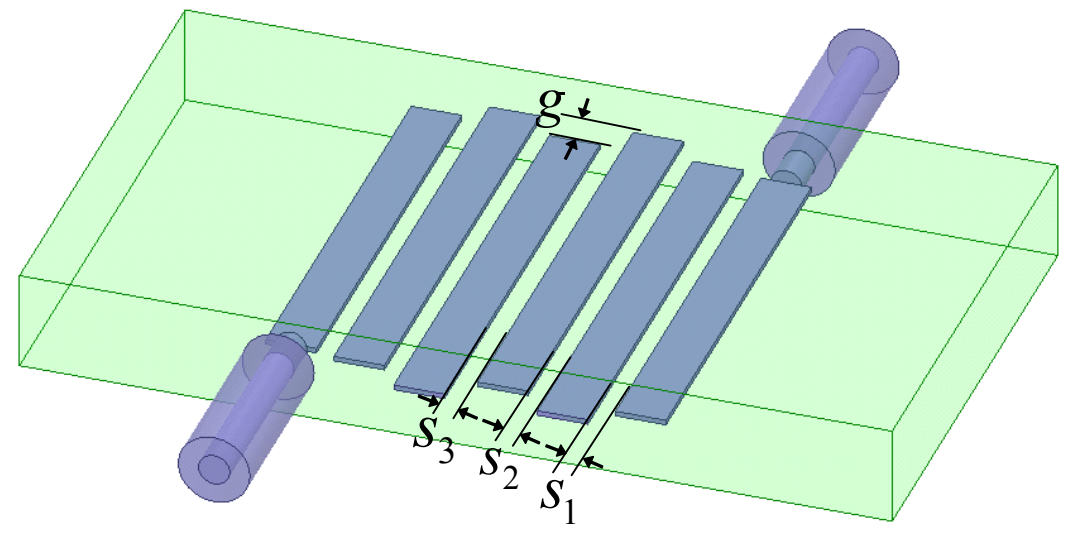

Figure 5.12: Structure of the inter-digital bandpass filter. The design variables for this example are $\boldsymbol{x}=\left[\begin{array}{llll}g & s_{1} & s_{2} & s_{3}\end{array}\right]$.

is from $0.7 \mathrm{~mm}$ to $3.1 \mathrm{~mm}$. The geometrical range for this example is

$$
\boldsymbol{X}=\left[\begin{array}{ll}
2.2 & 5.8 \\
0.5 & 2.9 \\
0.6 & 3 \\
0.7 & 3.1
\end{array}\right]
$$

Using a single neural network model is not enough to represent the behavior of the wide range responses. To build an accurate overall model, we use randomly distributed data generation method to generate the training and testing data. For this example, the number of training sample is 9000. The EM evaluation is performed by HFSS EM simulator. First order EM derivatives are obtained directly from the HFSS simulator. We use a cluster of Dell PowerEdge computers for parallel computation. After the parallel data generation, to decompose the entire geometrical 
ranges using our proposed decomposition technique, we first evaluated the secondorder EM derivative for each geometrical parameter. Based on the calculation method described in Section 5.2.2, the number of unknown elements in the Hessian matrix $N_{u}$ is 10 . To get good model accuracy, we use 15 neighboring samples (i.e., $\left.N_{h}=15\right)$ to calculate coefficients of Hessian matrix. After solving the Equation (5.9), we can get the second-order derivative of EM response w.r.t. four geometrical variables for each training data at each frequency point. The average values of secondorder derivative are calculate as $\left[\begin{array}{llll}Q_{1} & Q_{2} & Q_{3} & Q_{4}\end{array}\right]=\left[\begin{array}{llll}540.27 & 799.15 & 1169 & 861.18\end{array}\right]^{T}$.

Once we obtain the second-order derivatives, we can decompose the overall geometrical range into multiple sub-ranges. For the first training stage, i.e., $K=1$, the number of division $\boldsymbol{L}$ is calculated using Equation (5.20) as $\left[\begin{array}{llll}1 & 1 & 2 & 2\end{array}\right]$. This means for the first training stage, only the geometrical parameters $s_{2}$ and $s_{3}$ are decomposed. The total number of sub models is $n=4$ for the first training stage. Using the second-order derivative information, we can obtain the specific sub-range for each sub-model using the proposed decomposition process in Fig. 5.1. In this example, we define the overlapping coefficient $\delta=5 \%$. The sub-range for each sub-model after decomposition process is shown in Fig. 5.13.

Based on the sub-model range shown in Fig. 5.13, we can select the training and testing data for each sub-model from the overall training and testing data sets. To train the sub-model efficiently, in this example, a three-layer ANN with 50 hidden neurons is used to train the sub-models for all the training stages. We train the submodels using the parallel training technique to accelerate the training process. After the parallel training process is finished, we combine all the independently trained 


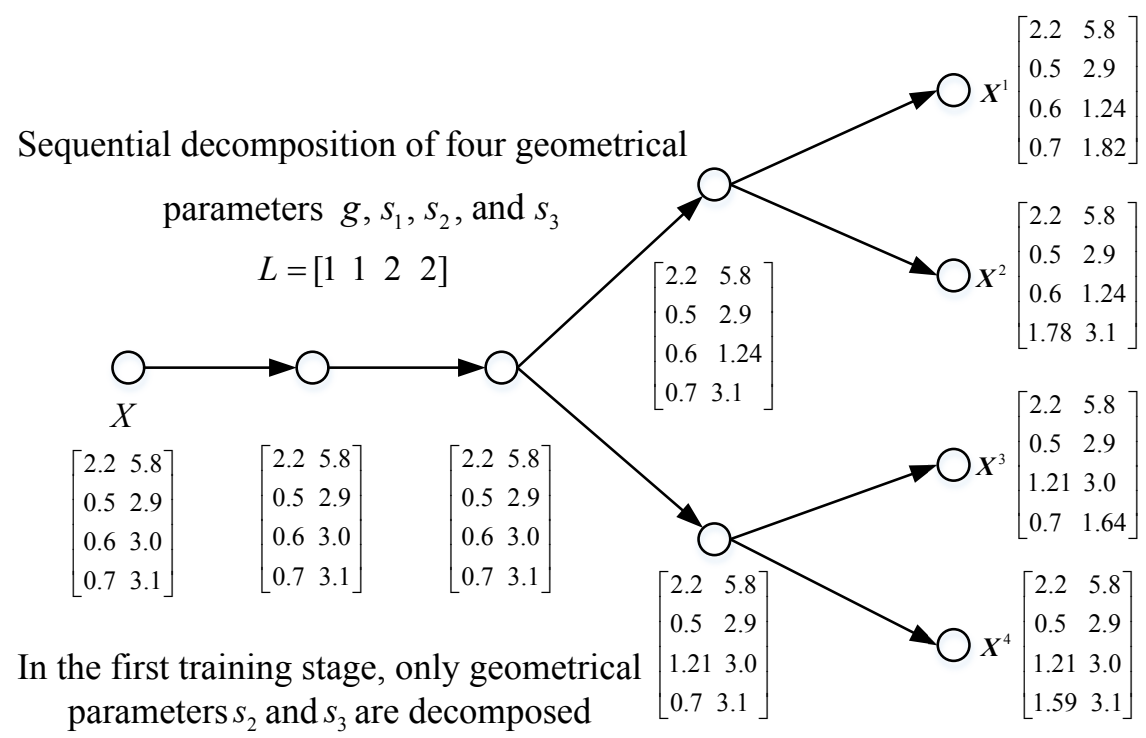

Figure 5.13: The sub-range for each sub-model after decomposition process at first training stage for the inter-digital bandpass filter example. The design parameters for this example are $\boldsymbol{x}=\left[\begin{array}{llll}g & s_{1} & s_{2} & s_{3}\end{array}\right]$. At the first training stage, the number of division $\boldsymbol{L}$ is calculated as $\left[\begin{array}{llll}1 & 1 & 2 & 2\end{array}\right]$. This means only the geometrical parameters $s_{2}$ and $s_{3}$ are decomposed at this stage. At the first and second level decomposition, the entire wide range is not decomposed. At the third level decomposition, the range geometrical parameter $s_{2}$ is decomposed into 2 sub-ranges. At the fourth level decomposition, the range geometrical parameter $s_{3}$ is decomposed into 2 subranges. The total number of sub-models is $n=1 \cdot 1 \cdot 2 \cdot 2=4$.

sub-models to obtain the overall model which covers the entire wide geometrical range. We should not simply combine all the sub-models because of the discontinuity problem at the boundary between adjacent sub-models. To solve the discontinuity problem to obtain the continuous overall model over the entire range, the proposed parallel modification process is performed for each trained sub-model following the flowchart in Fig. 5.4. 
After we perform the modification process for all the sub-models, the last step is to add all the modified sub-models together to get the overall model which covers the entire geometrical parameter range. We use all the training and testing data to test the overall model. The average training error for the overall model is $3.27 \%$, while the average testing error is $3.26 \%$. The user-defined threshold for the testing error is below $2 \%$. The overall model after the frist training stage still cannot satisfy the required error.

For the second training stage, i.e., $K=2$, the number of division $\boldsymbol{L}$ is calculated as [2 $\left.3 \begin{array}{lll}2 & 4\end{array}\right]$. The total number of sub models is $n=72$ for the second training stage. Using the second-order derivative information, we can obtain the specific sub-range for each sub-model using the proposed decomposition process in Fig. 5.1. Based on the sub-range for each sub-model, we can select the training and testing data for each sub-model from the overall training and testing data sets. We can train the sub-models using the parallel training technique to accelerate the training process. After the parallel training process is finished, we combine all the independently trained sub-models into one overall model to obtain the overall model which covers the entire wide geometrical range. We should not simply combine all the sub-models because of the discontinuity problem at the boundary between adjacent sub-models. To solve the discontinuity problem to obtain the continuous overall model over the entire range, the proposed parallel modification process is performed for each trained sub-model following the flowchart in Fig. 5.4.

After we perform the modification process for all the sub-models, the last step is to add all the modified sub-models together to get the overall model which covers 
the entire geometrical parameter range. We use all the training and testing data to test the overall model. The average training error for the overall model is $1.49 \%$, while the average testing error is $1.59 \%$. The overall model after second training stage satisfies the required error threshold. After second training stage, using our proposed decomposition technique, we build the accurate and continuous wide range parametric model which consists of 72 sub-models.

For comparison purpose, we also use the standard modeling methods using a single model to cover the entire wide geometrical range. A single ANN model is directly trained to learn the entire range of the geometrical parameters for four cases: case 1 being with 50 hidden neurons, case 2 being with 100 hidden neurons, case 3 being with 200 hidden neurons and case 4 being with 400 hidden neurons. Table 5.2 compares the different parametric modeling methods in terms of ANN structures, average training and testing error, and CPU time. From the table, we can see that our proposed model combining many sub-models is more accurate than the standard ANN model using a single ANN for the entire wide range modeling. The reason is that the proposed technique focuses on learning each small sub-region by a separate sub-model, while the standard modeling technique using a single ANN for the entire wide range modeling needs to compromise the ANN accuracy between different regions. Also our proposed technique uses less CPU time to achieve the accurate overall model since we incorporate the parallel training technique.

The comparison of the magnitude (in decibels) of $S_{11}$ between three models (the proposed model, ANN model with 50 hidden neurons, and ANN model with 400 hidden neurons) for two different filter test geometries are shown in Fig. 5.19. The 
Table 5.2: Comparisons of Different Methods for Parametric Modeling of the InterDigital Bandpass Example

\begin{tabular}{|c|c|c|c|c|}
\hline $\begin{array}{l}\text { Modeling } \\
\text { Method }\end{array}$ & $\begin{array}{c}\text { No. of Hidden } \\
\text { Neurons }\end{array}$ & $\begin{array}{c}\text { Average } \\
\text { Training Error }\end{array}$ & $\begin{array}{c}\text { Average Testing } \\
\text { Error }\end{array}$ & $\begin{array}{l}\text { CPU } \\
\text { Time }\end{array}$ \\
\hline ANN Model ${ }^{+}$ & 50 & $5.12 \%$ & $5.10 \%$ & $5.44 h$ \\
\hline $\mathrm{ANN} \mathrm{Model}^{+}$ & 100 & $4.84 \%$ & $4.83 \%$ & $11.1 h$ \\
\hline ANN Model ${ }^{+}$ & 200 & $4.17 \%$ & $4.17 \%$ & $22.56 h$ \\
\hline ANN Model ${ }^{+}$ & 400 & $3.97 \%$ & $3.96 \%$ & $45.68 h$ \\
\hline $\begin{array}{c}\text { Proposed Model at Training } \\
\text { Stage } 1 \text { Without Parallel }\end{array}$ & $\begin{array}{c}50 \\
4 \text { sub-models }\end{array}$ & $3.27 \%$ & $3.26 \%$ & $5.63 h$ \\
\hline $\begin{array}{c}\text { Proposed Model at Training } \\
\text { Stage } 2 \text { Without Parallel }\end{array}$ & $\begin{array}{c}50 \\
72 \text { sub-models } \\
\end{array}$ & $1.49 \%$ & $1.59 \%$ & $6.13 h$ \\
\hline $\begin{array}{c}\text { Final Proposed Model } \\
\text { Without Parallel* }\end{array}$ & $\begin{array}{c}50 \\
72 \text { sub-models }\end{array}$ & $1.49 \%$ & $1.59 \%$ & $11.76 h$ \\
\hline $\begin{array}{c}\text { Proposed Model at Training } \\
\text { Stage } 1 \text { With Parallel }\end{array}$ & $\begin{array}{c}50 \\
4 \text { sub-models }\end{array}$ & $3.27 \%$ & $3.26 \%$ & $2.53 h$ \\
\hline $\begin{array}{c}\text { Proposed Model at Training } \\
\text { Stage } 2 \text { With Parallel }\end{array}$ & $\begin{array}{c}50 \\
72 \text { sub-models }\end{array}$ & $1.49 \%$ & $1.59 \%$ & $0.34 h$ \\
\hline $\begin{array}{c}\text { Final Proposed Model } \\
\text { With Parallel }{ }^{\dagger}\end{array}$ & $\begin{array}{c}50 \\
72 \text { sub-models }\end{array}$ & $1.49 \%$ & $1.59 \%$ & $2.87 h$ \\
\hline
\end{tabular}

+ Standard ANN model using a single ANN for the entire wide range modeling

* Final result of proposed model without parallel after 2 stages of training

$\dagger$ Final result of proposed model with parallel after 2 stages of training

two test samples are from testing data and have never been used in training process. The values of the two test samples are as follows:

Test sample \#1: $\boldsymbol{x}=\left[\begin{array}{llll}3.9775 & 1.10502 .3500 & 2.9500\end{array}\right]^{T} \mathrm{~mm}$ 
Test sample \#2: $\boldsymbol{x}=\left[\begin{array}{llll}3.8125 & 1.7950 & 2.9500 & 2.1050\end{array}\right]^{T} \mathrm{~mm}$.

We use several graphs to demonstrate the continuity of the proposed overall model. Fig. 5.15 (a) shows the real and imaginary parts of $S_{11}$ along the direction of $g$. The values of the other parameters are fixed as $s_{1}=1.5 \mathrm{~mm}, s_{2}=2.4 \mathrm{~mm}$, $s_{3}=1.2 \mathrm{~mm}$, and $f=1.85 \mathrm{GHz}$. Fig. 5.15 (b) shows the real and imaginary parts of $S_{11}$ along the diagonal direction of $g$ and $s_{3}$. The values of the other parameters are fixed as $s_{1}=1.5 \mathrm{~mm}, s_{2}=2.4 \mathrm{~mm}$, and $f=1.91 \mathrm{GHz}$. Fig. 5.15 (c) shows the real and imaginary parts of $S_{11}$ along the diagonal direction of $g, s_{2}$, and $s_{3}$. The values of the other parameters are fixed as $s_{1}=1.5 \mathrm{~mm}$ and $f=1.81 \mathrm{GHz}$. Fig. 5.15 (d) shows the real and imaginary parts of $S_{11}$ along the diagonal direction of $g$, $s_{1}, s_{2}$, and $s_{3}$ at $1.84 \mathrm{GHz}$. From the figure, we can see that our proposed technique provides accurate and also continuous solutions compared to the HFSS simulation results across the boundaries along various directions.

Since the proposed wide range parametric model is accurate and continuous, we can implement the trained model into the design optimization where the design parameters can be repetitively adjusted during optimization. We perform the design optimization for the inter-digital bandpass filter with the design specification:

$$
\left|S_{11}\right| \leq-26 \mathrm{~dB}, 1.25 \mathrm{GHz} \leq f \leq 1.85 \mathrm{GHz}
$$

The design variables are the model inputs $\boldsymbol{x}=\left[\begin{array}{llll}g & s_{1} & s_{2} & s_{3}\end{array}\right]$. The initial values are $\boldsymbol{x}=\left[\begin{array}{llll}3.5 & 0.6 & 0.8 & 1.6\end{array}\right]^{T} \mathrm{~mm}$. This initial point actually lies in the subrange of the sub-model 2. The design optimization using the proposed overall model takes only about 30 seconds to achieve the optimal design solution for the 


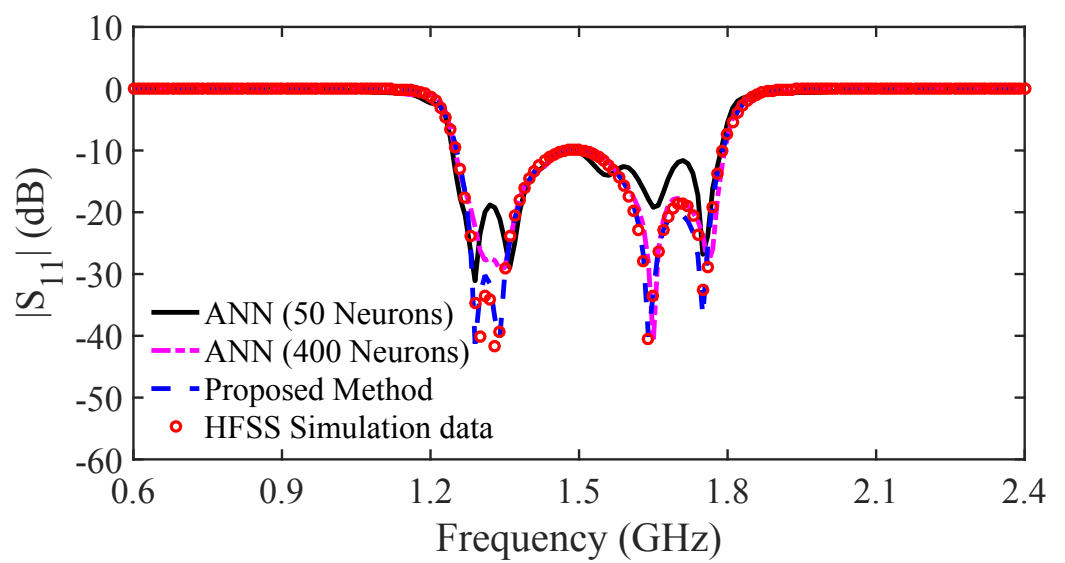

(a)

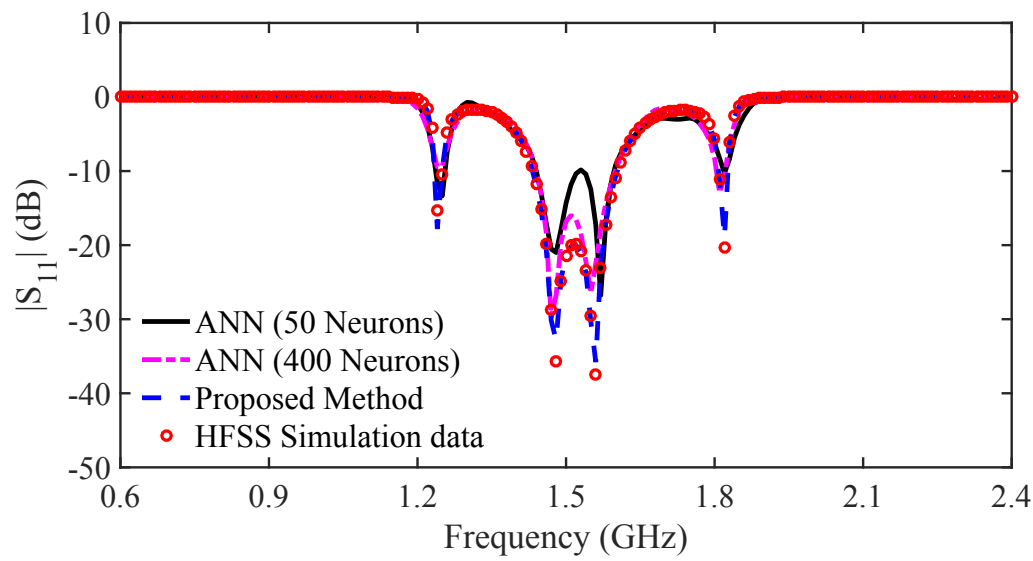

(b)

Figure 5.14: Comparison of the magnitude (in decibels) of $S_{11}$ of the overall parametric models developed using different modeling methods and HFSS simulation data: (a) test sample \#1 and (b) test sample \#2 for the inter-digital bandpass filter example. Our proposed model combining many sub-models is more accurate than standard ANN model using a single ANN for the entire wide range modeling because the proposed technique focuses on learning each small sub-region by a separate sub-model, while the standard modeling technique using a single ANN for the entire wide range modeling needs to compromise the ANN accuracy between different regions. 


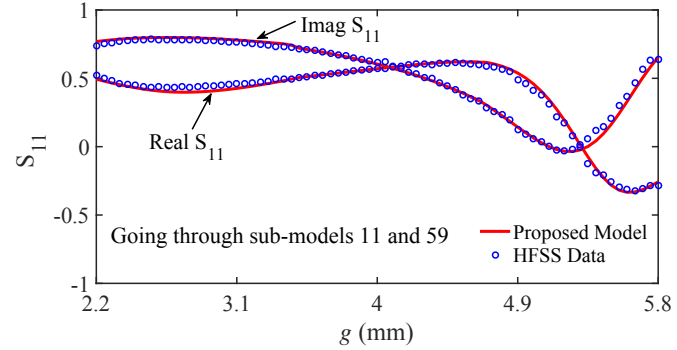

(a)

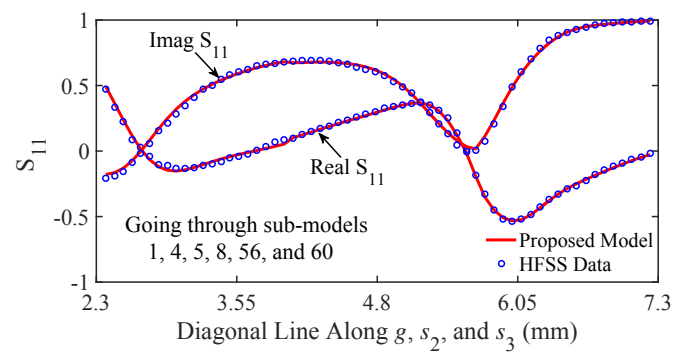

(c)

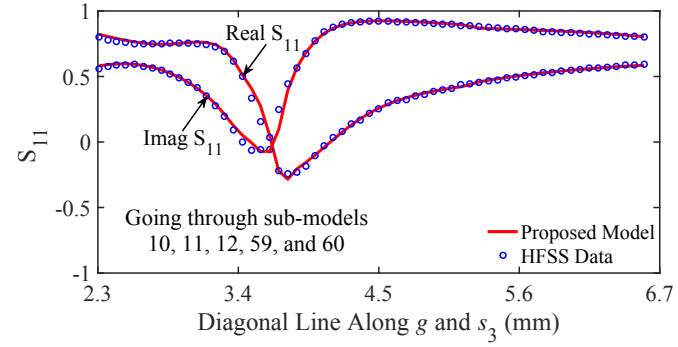

(b)

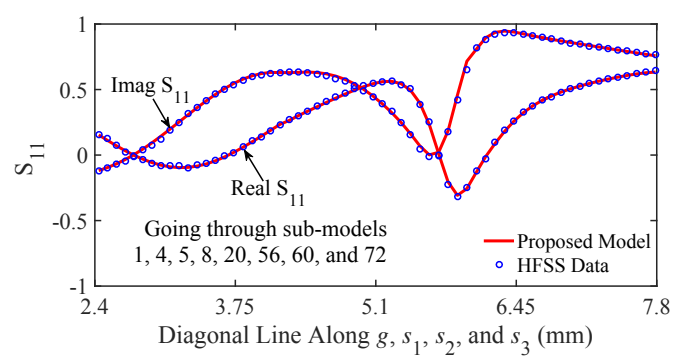

(d)

Figure 5.15: Demonstration of the continuity of the proposed overall model with design variables $\boldsymbol{x}=\left[\begin{array}{llll}g & s_{1} & s_{2} & s_{3}\end{array}\right]$. The real and imaginary parts of $S_{11}$ (a) along the direction of $g$ going through sub-models 11 and 59; (b) along the diagonal direction of $g$ and $s_{3}$ going through sub-models 10,11, 12, 59, and 60; (c) along the diagonal direction of $g, s_{2}$, and $s_{3}$ going through sub-models $1,4,5,8,56$, and 60; and (d) along the diagonal direction of $g, s_{1}, s_{2}$, and $s_{3}$ going through sub-models $1,4,5,8$, $20,56,60$, and 72 . The proposed technique provides accurate and also continuous solutions along different directions. Our solutions are verified with HFSS simulation results.

specification. The optimized design parameter values for the inter-digital bandpass filter are $\boldsymbol{x}=\left[\begin{array}{llll}3.13583 & 0.578552 & 1.4543 & 1.69661\end{array}\right]^{T} \mathrm{~mm}$. This optimized solution point lies in the sub-range of the sub-model 8 instead of sub-model 2. The HFSS simulations at the initial point and the optimal solution are shown in Fig. 5.21. Our proposed model behaves well in design optimization where the optimization moves freely and continuously between different sub-models. 


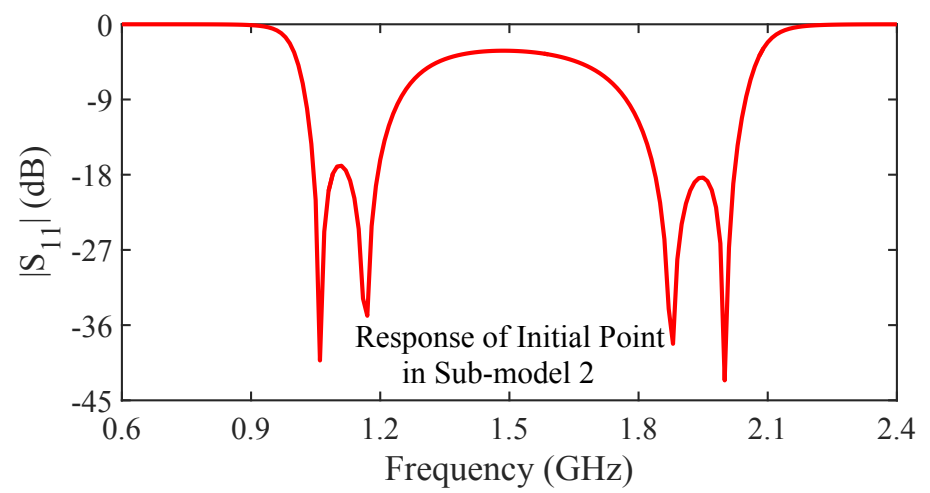

(a)

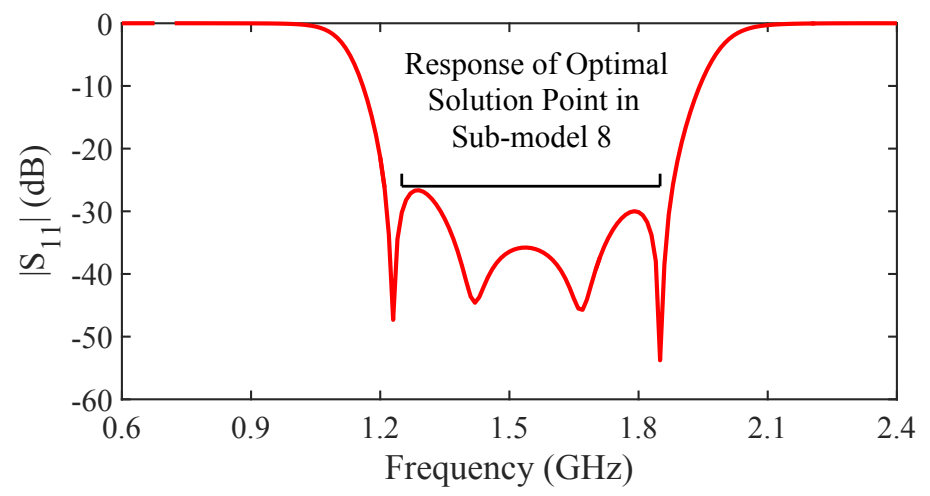

(b)

Figure 5.16: The proposed parametric model is used for design optimization for the inter-digital bandpass filter. The optimal solution is found by optimizing design variables $\left[g s_{1} s_{2} s_{3}\right]$ using our proposed model. The optimal solution is verified by HFSS simulation. The magnitude (in decibels) of $S_{11}$ of HFSS simulation data at (a) initial point in sub-model 2 and (b) optimal solution point in sub-model 8. Our proposed model behaves well in design optimization where the optimization moves freely and continuously between different sub-models.

\subsubsection{Parametric Modeling of a Four-Pole Waveguide Filter Incorporating Decomposition Technique}

We consider a four-pole waveguide filter [127] with tuning elements as the posts of square cross section placed at the center of each cavity and each coupling window, 


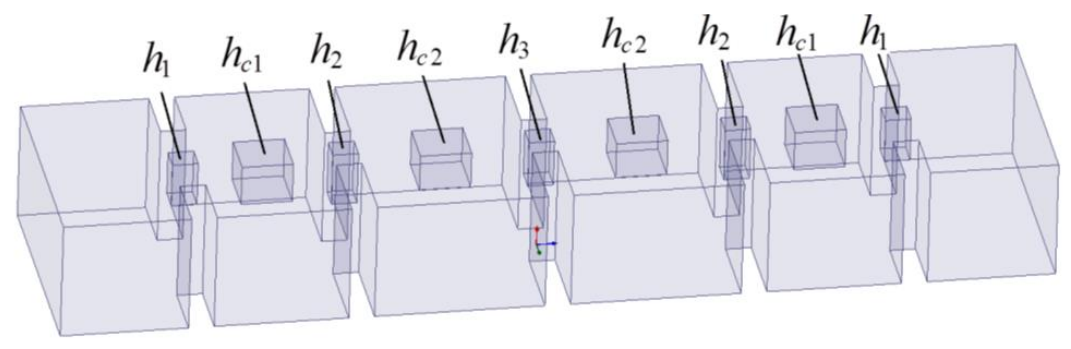

Figure 5.17: Structure of the four-pole waveguide filter. The design variables for this example are $\boldsymbol{x}=\left[h_{1} h_{2} h_{3} h_{c 1} h_{c 2}\right]$.

as shown in Fig. 5.17. For this example, height $h_{1}$, height $h_{2}$, and height $h_{3}$ are the heights of the tuning posts in the coupling windows. Heights $h_{c 1}$ and $h_{c 2}$ are the heights of the square cross section placed in the center of the resonator cavities. The input and output waveguides, as well as the resonant cavities, are standard WR-75 waveguides $(a=19.05 \mathrm{~mm}$ and $b=9.525 \mathrm{~mm})$. The thickness of the coupling windows is set to $2 \mathrm{~mm}$. The design variables for this example are $\boldsymbol{x}=\left[\begin{array}{lllll}h_{1} & h_{2} & h_{3} & h_{c 1} & h_{c 2}\end{array}\right]$. Frequency $f$ is an additional input. The model has two outputs, i.e., $R S_{11}$ and $I S_{11}$ which are the real and imaginary parts of the overall model output $S_{11}$ w.r.t. different values of geometrical input parameters.

For this example, the frequency range is from $10 \mathrm{GHz}$ to $12 \mathrm{GHz}$. The number of frequency points is 201. The range of the design variable $h_{1}$ is from $3.08 \mathrm{~mm}$ to $3.8 \mathrm{~mm}$, the range of the design variable $h_{2}$ is from $3.96 \mathrm{~mm}$ to $4.92 \mathrm{~mm}$, the range of the design variable $h_{3}$ is from $3.63 \mathrm{~mm}$ to $4.35 \mathrm{~mm}$, the range of the design variable $h_{c 1}$ is from $2.82 \mathrm{~mm}$ to $3.42 \mathrm{~mm}$, and the range of the design variable $h_{c 2}$ 
is from $2.65 \mathrm{~mm}$ to $3.25 \mathrm{~mm}$. The geometrical range for this example is

$$
\boldsymbol{X}=\left[\begin{array}{ll}
3.08 & 3.8 \\
3.96 & 4.92 \\
3.63 & 4.35 \\
2.82 & 3.42 \\
2.65 & 3.25
\end{array}\right] .
$$

Using a single neural network model is not enough to represent the behavior of the wide range responses. To build an accurate overall model, we use randomly distributed data generation method to generate the training and testing data. For this example, the number of training sample is 12000. The EM evaluation is performed by HFSS EM simulator. First order EM derivatives are obtained directly from the HFSS simulator. We use a cluster of Dell PowerEdge computers for parallel computation. After the parallel data generation, to decompose the entire geometrical ranges using our proposed decomposition technique, we first evaluated the second-order EM derivative for each geometrical parameter. Based on the calculation method described in Section 5.2.2, the number of unknown elements in the Hessian matrix $N_{u}$ is 15 . To get good model accuracy, we use 20 neighboring samples (i.e., $N_{h}=20$ ) to calculate coefficients of Hessian matrix. After solving the Equation (5.9), we can get the second-order derivative of EM response w.r.t. four geometrical variables for each training data at each frequency point. The average values of second-order derivative are calculate as $\left[\begin{array}{lllll}Q_{1} & Q_{2} & Q_{3} & Q_{4} & Q_{5}\end{array}\right]=\left[\begin{array}{lllll}1752.3 & 1407.6 & 1721.4 & 2316.9 & 3916.8\end{array}\right]^{T}$. 
Once we obtain the second-order derivatives, we can decompose the overall geometrical range into multiple sub-ranges. For the first training stage, i.e., $K=1$, the number of division $\boldsymbol{L}$ is calculated using Equation (5.20) as $\left[\begin{array}{llll}1 & 1 & 1 & 2\end{array}\right]$. This means for the first training stage, only the geometrical parameters $h_{c 1}$ and $h_{c 2}$ are decomposed. The total number of sub models is $n=6$ for the first training stage. Using the second-order derivative information, we can obtain the specific sub-range for each sub-model using the proposed decomposition process in Fig. 5.1. In this example, we define the overlapping coefficient $\delta=5 \%$. The sub-range for each sub-model after decomposition process is shown in Fig. 5.18.

Based on the sub-model range shown in Fig. 5.18, we can select the training and testing data for each sub-model from the overall training and testing data sets. To train the sub-model efficiently, in this example, a three-layer ANN with 50 hidden neurons is used to train the sub-models for all the training stages. We train the sub-models using the parallel training technique to accelerate the training process. After the parallel training process is finished, we need to combine all the independently trained sub-models to obtain the overall model which covers the entire wide geometrical range. We should not simply combine all the sub-models because of the discontinuity problem at the boundary between adjacent sub-models. To solve the discontinuity problem to obtain the continuous overall model over the entire range, the proposed parallel modification process is performed for each trained sub-model following the flowchart in Fig. 5.4.

After we perform the modification process for all the sub-models, the last step is to add all the modified sub-models together to get the overall model which covers 


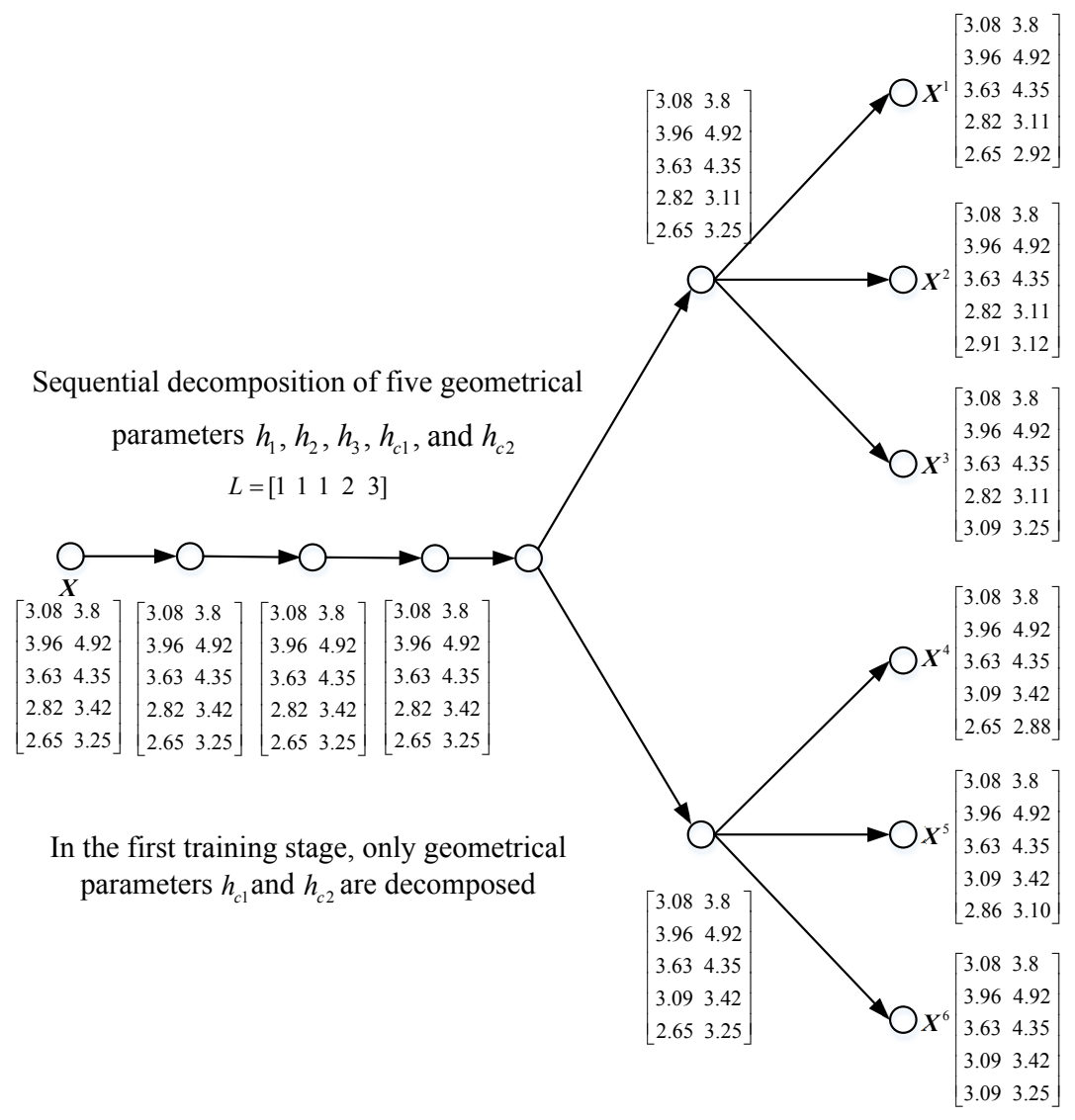

Figure 5.18: The sub-range for each sub-model after decomposition process at first training stage for the four-pole waveguide filter example. The design parameters for this example are $\boldsymbol{x}=\left[\begin{array}{lllll}h_{1} & h_{2} & h_{3} & h_{c 1} & h_{c 2}\end{array}\right]$. At the first training stage, the number of division $\boldsymbol{L}$ is calculated as $\left[\begin{array}{llll}1 & 1 & 1 & 2\end{array}\right]$. This means only the geometrical parameters $h_{c 1}$ and $h_{c 2}$ are decomposed at this stage. At the first, second, and third level decomposition, the entire wide range is not decomposed. At the fourth level decomposition, the range geometrical parameter $h_{c 1}$ is decomposed into 2 subranges. At the fifth level decomposition, the range geometrical parameter $h_{c 2}$ is decomposed into 3 sub-ranges. The total number of sub-models is $n=1 \cdot 1 \cdot 1 \cdot 2 \cdot 3=6$.

the entire geometrical parameter range. We use all the training and testing data to test the overall model. The average training error for the overall model is $2.51 \%$, 
while the average testing error is $2.52 \%$. The user-defined threshold for the testing error is below $2 \%$. The overall model after the frist training stage still cannot satisfy the required error.

For the second training stage, i.e., $K=2$, the number of division $\boldsymbol{L}$ is calculated as [ $\left[\begin{array}{llll}2 & 2 & 2 & 3\end{array}\right.$ 5]. The total number of sub models is $n=120$ for the second training stage. Using the second-order derivative information, we can obtain the specific sub-range for each sub-model using the proposed decomposition process in Fig. 5.1. Based on the sub-range for each sub-model, we can select the training and testing data for each sub-model from the overall training and testing data sets. We can train the sub-models using the parallel training technique to accelerate the training process. After the parallel training process is finished, we need to combine all the independently trained sub-models into one overall model to obtain the overall model which covers the entire wide geometrical range. We should not simply combine all the sub-models because of the discontinuity problem at the boundary between adjacent sub-models. To solve the discontinuity problem to obtain the continuous overall model over the entire range, the proposed parallel modification process is performed for each trained sub-model following the flowchart in Fig. 5.4.

After we perform the modification process for all the sub-models, the last step is to add all the modified sub-models together to get the overall model which covers the entire geometrical parameter range. We use all the training and testing data to test the overall model. The average training error for the overall model is $1.09 \%$, while the average testing error is $1.08 \%$. The overall model after second training stage satisfies the required error threshold. After second training stage, using our 
proposed decomposition technique, we build the accurate and continuous wide range parametric model which consists of 120 sub-models.

For comparison purpose, we also use the standard modeling methods using a single model to cover the entire wide geometrical range. A single ANN model is directly trained to learn the entire range of the geometrical parameters for four cases: case 1 being with 50 hidden neurons, case 2 being with 100 hidden neurons, case 3 being with 200 hidden neurons and case 4 being with 400 hidden neurons. Table 5.3 compares the different parametric modeling methods in terms of ANN structures, average training and testing error, and CPU time. From the table, we can see that our proposed model combining many sub-models is more accurate than the standard ANN model using a single ANN for the entire wide range modeling. The reason is that the proposed technique focuses on learning each small sub-region by a separate sub-model, while the standard modeling technique using a single ANN for the entire wide range modeling needs to compromise the ANN accuracy between different regions. Also our proposed technique uses less CPU time to achieve the accurate overall model since we incorporate the parallel training technique.

The comparison of the magnitude (in decibels) of $S_{11}$ between three models (the proposed model, ANN model with 50 hidden neurons, and ANN model with 400 hidden neurons) for two different filter test geometries are shown in Fig. 5.19. The two test samples are from testing data and have never been used in training process. The values of the two test samples are as follows:

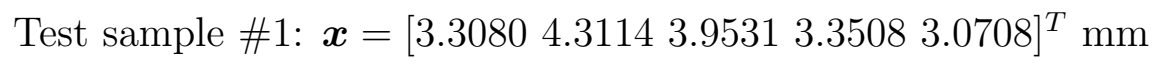


Table 5.3: Comparisons of Different Methods for Parametric Modeling of the FourPole Waveguide Filter Example

\begin{tabular}{|c|c|c|c|c|}
\hline Modeling Method & $\begin{array}{l}\text { No. of Hidden } \\
\text { Neurons }\end{array}$ & $\begin{array}{l}\text { Average } \\
\text { Training Error }\end{array}$ & $\begin{array}{c}\text { Average } \\
\text { Testing Error }\end{array}$ & $\begin{array}{l}\text { CPU } \\
\text { Time }\end{array}$ \\
\hline $\mathrm{ANN} \mathrm{Model}^{+}$ & 50 & $4.31 \%$ & $4.30 \%$ & $5.45 h$ \\
\hline $\mathrm{ANN}_{\text {Model }}{ }^{+}$ & 100 & $3.16 \%$ & $3.16 \%$ & $12.7 \mathrm{~h}$ \\
\hline ANN Model $^{+}$ & 200 & $3.11 \%$ & $3.12 \%$ & $34.23 h$ \\
\hline $\mathrm{ANN}_{\text {Model }}{ }^{+}$ & 400 & $2.77 \%$ & $2.76 \%$ & $71.88 h$ \\
\hline $\begin{array}{l}\text { Proposed Model at Training } \\
\text { Stage } 1 \text { Without Parallel }\end{array}$ & $\begin{array}{c}50 \\
6 \text { sub-models }\end{array}$ & $2.51 \%$ & $2.52 \%$ & $5.78 h$ \\
\hline $\begin{array}{c}\text { Proposed Model at Training } \\
\text { Stage } 2 \text { Without Parallel }\end{array}$ & $\begin{array}{c}50 \\
120 \text { sub-models }\end{array}$ & $1.09 \%$ & $1.08 \%$ & $6.29 h$ \\
\hline $\begin{array}{c}\text { Final Proposed Model } \\
\text { Without Parallel* }\end{array}$ & $\begin{array}{c}50 \\
120 \text { sub-models }\end{array}$ & $1.09 \%$ & $1.08 \%$ & $12.07 h$ \\
\hline $\begin{array}{l}\text { Proposed Model at Training } \\
\text { Stage } 1 \text { With Parallel }\end{array}$ & $\begin{array}{c}50 \\
6 \text { sub-models }\end{array}$ & $2.51 \%$ & $2.52 \%$ & $1.06 h$ \\
\hline $\begin{array}{l}\text { Proposed Model at Training } \\
\text { Stage } 2 \text { With Parallel }\end{array}$ & $\begin{array}{c}50 \\
120 \text { sub-models }\end{array}$ & $1.09 \%$ & $1.08 \%$ & $0.21 h$ \\
\hline $\begin{array}{c}\text { Final Proposed Model } \\
\text { With Parallel }{ }^{\dagger}\end{array}$ & $\begin{array}{c}50 \\
120 \text { sub-models }\end{array}$ & $1.09 \%$ & $1.08 \%$ & $1.27 \mathrm{~h}$ \\
\hline
\end{tabular}

+ Standard ANN model using a single ANN for the entire wide range modeling

* Final result of proposed model without parallel after 2 stages of training

$\dagger$ Final result of proposed model with parallel after 2 stages of training

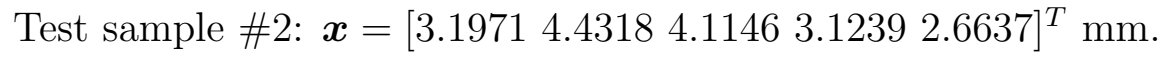

We use several graphs to demonstrate to the continuity of the proposed overall model. Fig. 5.20 (a) shows the real and imaginary parts of $S_{11}$ along the direction 


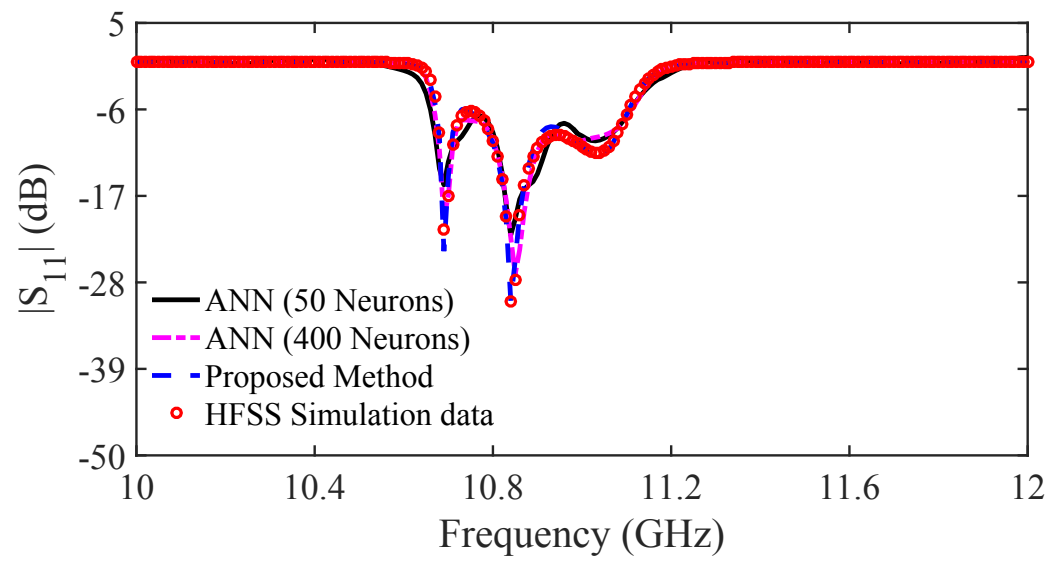

(a)

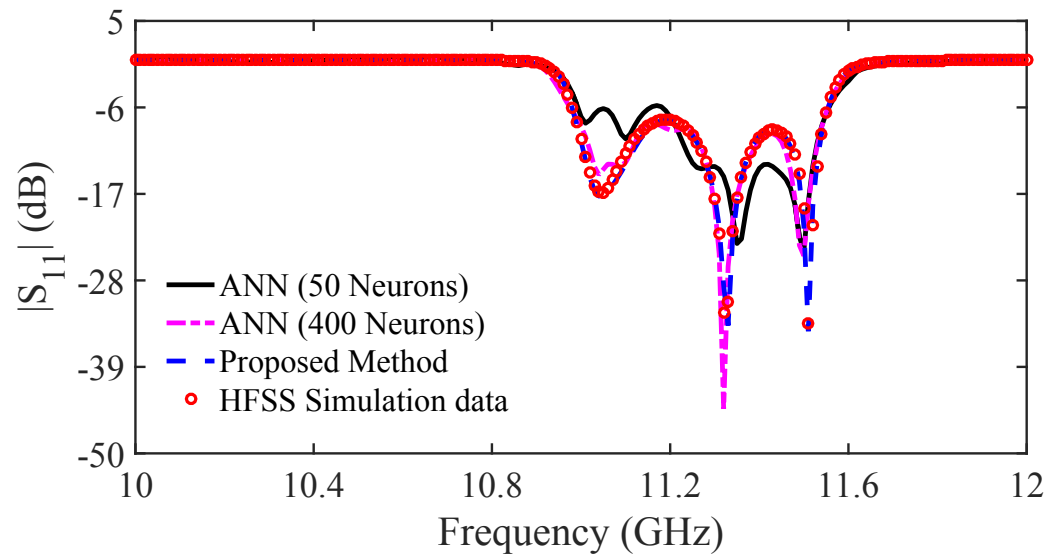

(b)

Figure 5.19: Comparison of the magnitude (in decibels) of $S_{11}$ of the overall parametric models developed using different modeling methods and HFSS simulation data: (a) test sample \#1 and (b) test sample \#2 for the four-pole waveguide filter example. Our proposed model combining many sub-models is more accurate than standard ANN model using a single ANN for the entire wide range modeling because the proposed technique focuses on learning each small sub-region by a separate sub-model, while the standard modeling technique using a single ANN for the entire wide range modeling needs to compromise the ANN accuracy between different regions. 
of $h_{1}$. The values of the other parameters are fixed as $h_{2}=4.5 \mathrm{~mm}, h_{3}=4.2 \mathrm{~mm}$, $h_{c 1}=3.1 \mathrm{~mm}, h_{c 2}=2.9 \mathrm{~mm}$, and $f=11.15 \mathrm{GHz}$. Fig. 5.20 (b) shows the real and imaginary parts of $S_{11}$ along the diagonal direction of $h_{1}$ and $h_{2}$. The values of the other parameters are fixed as $h_{3}=4.2 \mathrm{~mm}, h_{c 1}=3.1 \mathrm{~mm}, h_{c 2}=2.9 \mathrm{~mm}$, and $f=10.95 \mathrm{GHz}$. Fig. 5.20 (c) shows the real and imaginary parts of $S_{11}$ along the diagonal direction of $h_{1}, h_{2}$, and $h_{3}$. The values of the other parameters are fixed as $h_{c 1}=3.1 \mathrm{~mm}, h_{c 2}=2.9 \mathrm{~mm}$, and $f=10.75 \mathrm{GHz}$. Fig. 5.20 (d) shows the real and imaginary parts of $S_{11}$ along the diagonal direction of $h_{1}, h_{2}, h_{3}$, and $h_{c 1}$. The values of the other parameters are fixed as $h_{c 2}=2.9 \mathrm{~mm}$ and $f=10.8 \mathrm{GHz}$. Fig. 5.20 (e) shows the real and imaginary parts of $S_{11}$ along the diagonal direction of $h_{1}, h_{2}, h_{3}, h_{c 1}$, and $h_{c 2}$ at $10.84 \mathrm{GHz}$. From the figure, we can see that our proposed technique provides accurate and also continuous solutions compared to the HFSS simulation results across the boundaries along various directions.

Since the proposed wide range parametric model is accurate and continuous, we can implement the trained model into the design optimization where the design parameters can be repetitively adjusted during optimization. We perform the design optimization for the four-pole waveguide filter with the design specification:

$$
\left|S_{11}\right| \leq-26 \mathrm{~dB}, 10.85 \mathrm{GHz} \leq f \leq 11.15 \mathrm{GHz}
$$

The design variables for this example are the model inputs $\boldsymbol{x}=\left[h_{1} h_{2} h_{3} h_{c 1} h_{c 2}\right]$. The initial values are $\boldsymbol{x}=\left[\begin{array}{lllll}3.2 & 4.5 & 4.2 & 3.1 & 2.9\end{array}\right]^{T} \mathrm{~mm}$. This initial point actually lies in the sub-range of the sub-model 52. The design optimization using the proposed overall model takes only about 2 minutes to achieve the optimal design solution for 


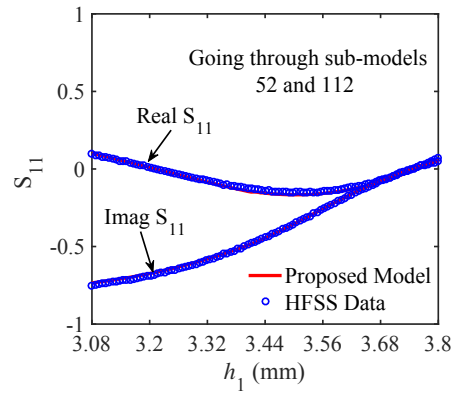

(a)

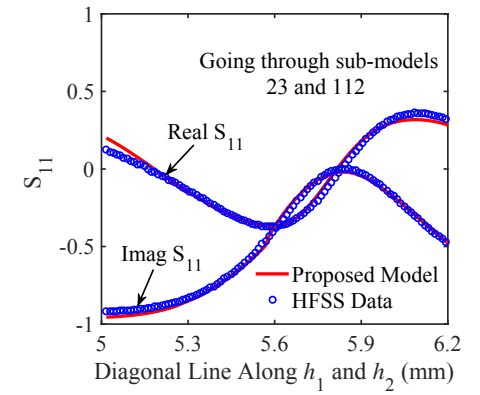

(b)

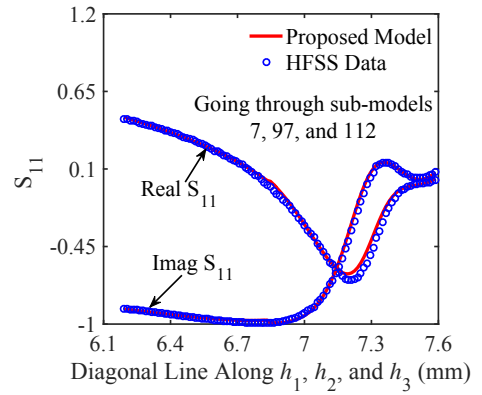

(c)

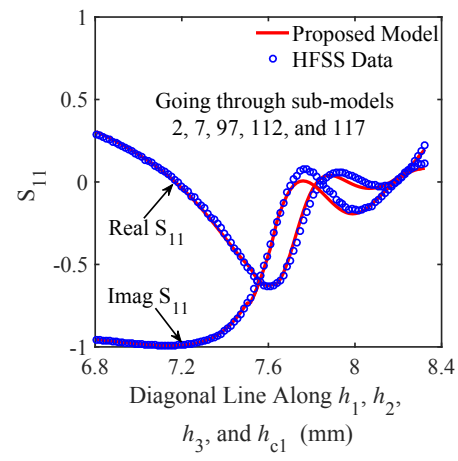

(d)

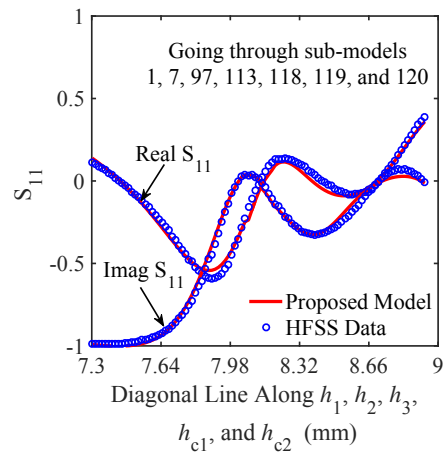

(e)

Figure 5.20: Demonstration of the continuity of the proposed overall model with design variables $\boldsymbol{x}=\left[\begin{array}{lllll}h_{1} & h_{2} & h_{3} & h_{c 1} & h_{c 2}\end{array}\right]$. The real and imaginary parts of $S_{11}$ (a) along the direction of $h_{1}$ going through sub-models 52 and 112; (b) along the diagonal direction of $h_{1}$ and $h_{2}$ going through sub-models 23 and 112; (c) along the diagonal direction of $h_{1}, h_{2}$, and $h_{3}$ going through sub-models 7,97 , and 112; (d) along the diagonal direction of $h_{1}, h_{2}, h_{3}$, and $h_{c 1}$ going through sub-models $2,7,97,112$, and 117; and (e) along the diagonal direction of $h_{1}, h_{2}, h_{3}, h_{c 1}$, and $h_{c 2}$ going through sub-models $1,7,97,113,118,119$, and 120 . The proposed technique provides accurate and also continuous solutions along different directions. Our solutions are verified with HFSS simulation results.

the specification. The optimized design parameter values for the four-pole waveguide filter are

$$
\boldsymbol{x}=\left[\begin{array}{lllll}
3.62972 & 4.39528 & 3.94808 & 3.22134 & 2.95377
\end{array}\right]^{T} \mathrm{~mm} .
$$


This optimized solution point lies in the sub-range of the sub-model 103 instead of sub-model 52. The HFSS simulations at the initial point and the optimal solution are shown in Fig. 5.21. Our proposed model behaves well in design optimization where the optimization moves freely and continuously between different sub-models.

\subsection{Conclusion}

We have proposed a novel decomposition technique to address the challenges of EM parametric modeling where the values of geometrical parameters change in a large range. A systematic and automated algorithm based on second-order derivative information has been implemented to decompose the overall geometrical range into a set of sub-ranges. An ANN model with simple structure has been developed with geometrical parameters as variables in each sub-region. A new technique has been proposed to combine the developed sub-models to obtain a continuous overall model by solving the multi-dimensional discontinuity problem. Parallel data generation, parallel sub-model training and parallel sub-model modification have been performed to speed up the model development process. The proposed technique has provided an efficient mathematical methodology to perform the decomposition so that the cumbersome decomposition process can be done systematically and automatically. Compared with standard modeling methods using a single model to cover the entire wide geometrical range, the proposed method has obtained better model-accuracy with short model-development time. Three microwave examples have been used to illustrate the validity of the proposed technique. 


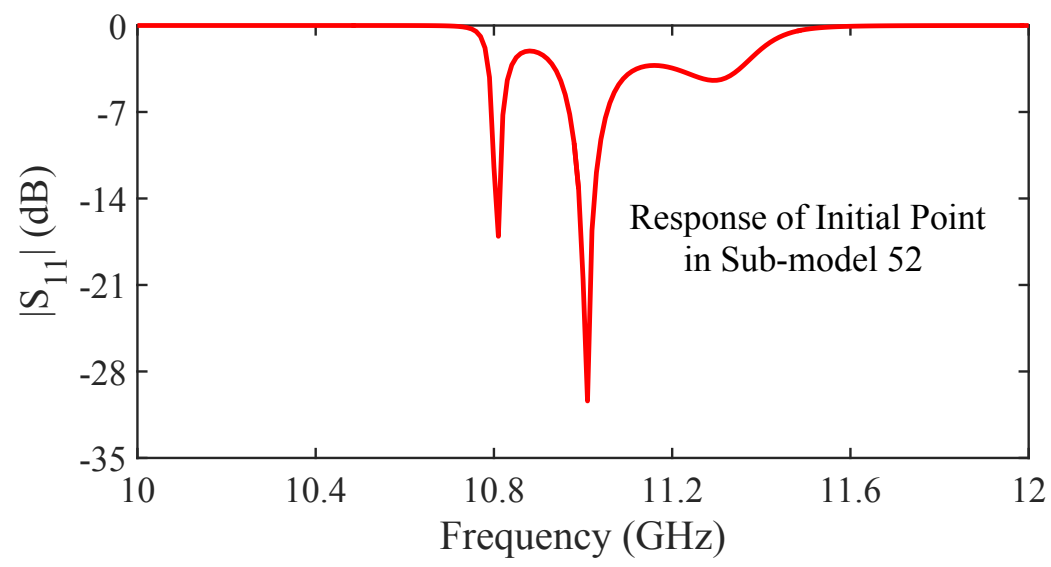

(a)

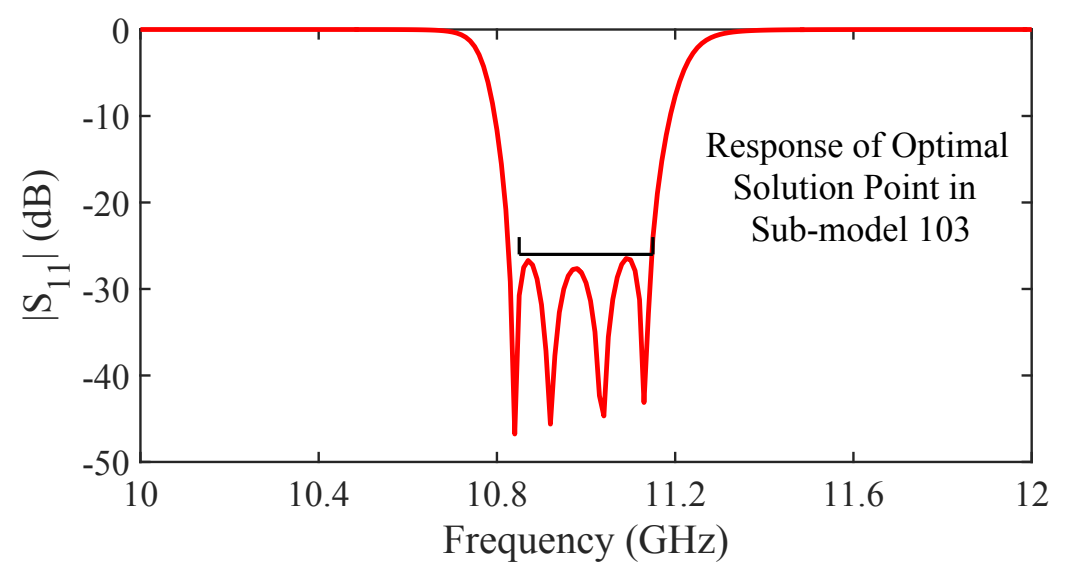

(b)

Figure 5.21: The proposed parametric model is used for design optimization for the four-pole waveguide filter. The optimal solution is found by optimizing design variables $\left[\begin{array}{lllll}h_{1} & h_{2} & h_{3} & h_{c 1} & h_{c 2}\end{array}\right]$ using our proposed model. The optimal solution is verified by HFSS simulation. The magnitude (in decibels) of $S_{11}$ of HFSS simulation data at (a) initial point in sub-model 52 and (b) optimal solution point in submodel 103. Our proposed model behaves well in design optimization where the optimization moves freely and continuously between different sub-models. 


\section{Chapter 6}

\section{Conclusions and Future Research}

\subsection{Conclusions}

In this thesis, several new techniques have been proposed to speed up the parametric modeling and optimization process of EM and multiphysics behaviors. A novel technique has been proposed to develop a low-cost EM centric multiphysics parametric model for microwave components. A novel parallel EM centric multiphysics optimization technique has been proposed to accelerate multiphysics design process. A further development of wide range parametric model has also been proposed.

In the first part of the thesis, a space mapped multiphysics parametric modeling technique has been proposed to develop an efficient multiphysics parametric model for microwave components. In the proposed method, we use the EM single physics (EM only) behaviors w.r.t different values of geometrical parameters in nondeformed structure of microwave components as the coarse model. Two mapping module functions have been formulated to map the EM domain responses to the multiphysics domain responses. Our proposed technique can achieve good accuracy 
of the multiphysics model with fewer multiphysics training data and less computational cost than direct multiphysics parametric modeling. After the proposed multiphysics modeling process, the trained multiphysics model can be used to provide accurate and fast prediction of multiphysics analysis responses of microwave components with geometrical and non-geometrical design parameters as variables. The developed overall model can be also used for high-level EM centric multiphysics design and optimization. We have used two microwave waveguide filter examples to illustrate our proposed method in the chapter. The proposed technique for multiphysics parametric model can be applied to other passive microwave component modeling with physical parameters as variables.

In our proposed parallel EM centric multiphysics optimization technique, poleresidue based transfer function has been exploited to build an effective and robust surrogate model. A group of modified quadratic mapping functions has been formulated to map the relationships between pole/residues of the transfer function and the design variables. Multiple EM centric multiphysics evaluations have been performed in parallel to generate the training data for establishing the surrogate model. The surrogate model is valid in a relatively large neighborhood which makes a large and effective optimization update in each optimization iteration. The trust region algorithm has been adopted to guarantee the convergence of the proposed multiphysics optimization algorithm. Our proposed multiphysics optimization technique has taken a small number of optimization iterations to obtain the optimal EM centric multiphysics response.

As a further advancement, we have proposed a novel decomposition technique 
to address the challenges of EM parametric modeling with geometrical changes in a large range. A systematic and automatic algorithm based on second-order derivative information has been implemented to decompose the modeling problem with a wide geometrical range into a set of sub-range modeling problems. Multiple sub-models has been independently developed with geometrical parameter as variables in their own sub-ranges. A new technique has been proposed to combine the developed sub-models to obtain the continuous overall model by solving the multi-dimensional discontinuity problem. Parallel data generation, parallel sub-model training and parallel sub-model modification have been performed to speed up the model development process. The proposed technique has provided an efficient mathematical methodology to perform the decomposition so that the cumbersome process can be done systematically and automatically. Compared with standard modeling methods using one model to cover the entire wide geometrical range, the proposed method has obtained better model-accuracy with short model-development time.

\subsection{Future Research}

Some of the future directions based on the expansion of the proposed parametric modeling and optimization techniques have been stated in this thesis as follows

- One of the future directions is to expand the proposed space mapping based multiphysics parametric modeling technique to guide the surrogate-based multiphysics optimization for the microwave components design. We can use the pure EM responses as the EM domain coarse model and incorporate the space 
mapping techniques to develop the surrogate model. After training the surrogate model, we can build a good surrogate model which can be valid in a relatively large neighborhood. Parallel multiphysics data generation can used to speed up the data generation time. Trust region can be further exploited to guarantee the convergence of the proposed technique. By using our proposed technique, we can make an effective and large optimization update in each optimization iteration so that we can reach the optimal solutions in fewer iterations so that the multiphysics design can be further accelerated.

- Another future direction is to expand the proposed multiphysics parametric modeling and optimization to multiphysics yield estimation and optimization. Yield optimization is an important topic for the fabrication process of microwave components. Manufactured outcomes are spread over a region around the nominal design. In order to improve the yield, the statistical design variables should be taken into consideration. For fabricating the microwave components, the multiphysics simulation is needed to consider the operations in a real-world multiphysics environment. By expanding our proposed multiphysics optimization to yield optimization, we can obtain better optimal design solutions and therefore the yield can be improved.

- Another possible direction is to expend the proposed decomposition techniques to multiphysics wide range parametric modeling and optimization. For the design of microwave components with various specifications, we can develop a wide range multiphysics parametric model by incorporating the decomposition 
techniques. We will propose a new technique to reduce the number of training samples for developing the wide range model. And as a further development, we can exploit the wide range model as the surrogate model to perform the surrogate based optimization. In this way, the update step for each iteration can be very large and we can obtain the optimal solution in very few iterations.

- Another further work is to expand the decomposition techniques to speed up the space mapping based optimization process with many design parameters. When there are many design variables, the mapping between the fine model and coarse model may be not easy to be formulated. Especially when the space mapping surrogate model developed in each iteration is trained to match the fine model at multiple points simultaneously. We propose a decomposition technique to decompose the design parameters into small subsets based on the second order derivative information to calculate the correlation information between design parameters. After decomposition, we have multiple suboptimizations in which sub-space mappings can be formulated. By solving the sub-space mapping optimization and combining the solved sub-solutions, we can obtain the updates for one iteration. The optimization updates of the proposed technique is much larger compared with the optimization without decomposition. Therefore, the proposed technique can reach the optimal solution in fewer iterations.

- As a further research, we can expend the surrogate based optimization techniques to the tunable microwave filter designs. For design the tunable filter, 
there are two sets of design parameters, one sets of the design parameters are the fixed geometrical parameters and the other sets are the tunable geometrical parameters. Our propose is to design the tunable filter to make it fit a wide frequency range. We first build a novel surrogate model to guide the optimization process to obtain optimal solutions for two different specifications at different frequency bands. In this optimization, the fixed geometrical parameters will be updated by considering both specifications while the tunable design parameters are updated freely based on two different specifications. After optimization is finished, we can obtain the values of fixed geometrical parameters and the range of the tunable parameters. By using our proposed technique, we can design a good optimal solution to obtain a wide range tunable filter. 


\section{Appendix A}

In this appendix, we provide the detailed calculation of $N_{u}$ where $N_{u}$ represents the number of unknown elements in the Hessian matrix. Since Hessian matrix is a symmetric matrix, i.e., $H_{i, j}^{k}=H_{j, i}^{k}, N_{u}$ can be calculated as

$$
N_{u}=\frac{1}{2} N(N+1)
$$

where $N$ is number of design variables. 


\section{Appendix B}

In this appendix, we provide the detailed derivation of Equation (5.9). The EM response at $\boldsymbol{x}^{v}, v \in I_{k}$ can be represented using local Taylor expansion as

$$
\begin{aligned}
R_{f}\left(\boldsymbol{x}^{v}, f\right) & =R_{f}\left(\boldsymbol{x}^{k}, f\right)+\left(\boldsymbol{g}^{k}\right)^{T}\left(\boldsymbol{x}^{v}-\boldsymbol{x}^{k}\right) \\
+ & \frac{1}{2}\left(\boldsymbol{x}^{v}-\boldsymbol{x}^{k}\right)^{T} \boldsymbol{H}^{k}\left(\boldsymbol{x}^{v}-\boldsymbol{x}^{k}\right), \quad v \in I_{k} .
\end{aligned}
$$

The Hessian matrix $\boldsymbol{H}^{k}$ contains elements of the quadratic term at the $k^{\text {th }}$ training sample. We rewrite the above equation as

$$
b^{k, v}=\left(\boldsymbol{a}^{k, v}\right) \cdot \boldsymbol{h}^{k}, v \in I_{k}
$$

where $\boldsymbol{a}^{k, v}$ and $b^{k, v}$ are given in Equations (5.10) and (5.12), respectively. By applying Equation (6.3) for all the samples $\boldsymbol{x}^{v}$ where $v \in I_{k}$, we can formulate $N_{h}$ linear equations as

$$
\boldsymbol{b}^{k}=\boldsymbol{A}^{k} \cdot \boldsymbol{h}^{k}
$$

where $\boldsymbol{b}^{k}$ is a $N_{h} \times 1$ vector containing $b^{k, v}$ for all the values of $v, v \in I_{k} . \boldsymbol{A}^{k}$ is a $N_{h} \times N_{u}$ matrix containing the row vectors of $\boldsymbol{a}^{k, v}$ for all the values of $v, v \in I_{k}$. Each row of Equation (6.4) represents the coefficients in Equation (6.3) for one sample close to $\boldsymbol{x}^{k}$, say sample $\boldsymbol{x}^{v}, v \in I_{k}$. The total number of samples is $N_{h}$ and 
hence Equation (6.4) has $N_{h}$ rows for calculating the second-order derivative at the $k^{\text {th }}$ training sample. The linear system in Equation (6.4) can be solved by using the following equation as

$$
\boldsymbol{h}^{k}=\left(\left(\boldsymbol{A}^{k}\right)^{T} \boldsymbol{A}^{k}\right)^{-1}\left(\boldsymbol{A}^{k}\right)^{T} \boldsymbol{b}^{k}
$$

considering $\boldsymbol{A}^{k}$ is not a square matrix. 


\section{Appendix C}

In this appendix, we provide the detailed calculation of $t_{u}$ used in Equations (5.37) and (5.38). For the sub-model $t$, we can obtain the index vector $\boldsymbol{\lambda}^{t}$ using Equation (5.30), formulated as

$$
\boldsymbol{\lambda}^{t}=\left[\begin{array}{llll}
\lambda_{1}^{t} & \lambda_{2}^{t} & \cdots & \lambda_{N}^{t}
\end{array}\right]^{T}
$$

Since $t_{u}$ is the index number of the neighboring sub-model bordering at the upper side of the $t^{t h}$ sub-model along the direction of $x_{i}$, the index number $\lambda_{j}^{t_{u}}$ for submodel $t_{u}$ can be calculated from that for sub-model $t$ as

$$
\lambda_{j}^{t_{u}}=\left\{\begin{array}{l}
\lambda_{j}^{t}, j \neq i \\
\lambda_{j}^{t}+1, j=i
\end{array}\right.
$$

for $j \in\{1,2, \cdots, N\}$. According to Equation (6.7), the value of index vector $\boldsymbol{\lambda}^{t_{u}}$ for sub-model $t_{u}$ is calculated as

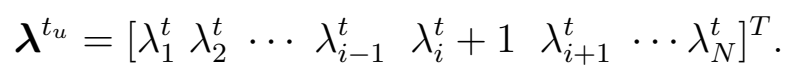


Since the $\boldsymbol{\lambda}^{t_{u}}$ and the sub-model index number $t_{u}$ has an one-to-one correspondence, we can calculate $t_{u}$ from $\boldsymbol{\lambda}^{t_{u}}$ as

$$
t_{u}=1+\sum_{j=1}^{N}\left(\left(\lambda_{j}^{t_{u}}-1\right) \cdot \prod_{p=j+1}^{N} L_{p}\right)
$$




\section{Appendix D}

In this appendix, we provide the detailed calculation of $t_{l}$ used in Equations (5.40) and (5.41). For the sub-model $t$, we can obtain the index vector $\boldsymbol{\lambda}^{t}$ using Equation (5.30), formulated as

$$
\boldsymbol{\lambda}^{t}=\left[\begin{array}{llll}
\lambda_{1}^{t} & \lambda_{2}^{t} & \cdots & \lambda_{N}^{t}
\end{array}\right]^{T}
$$

Since $t_{l}$ is the index number of the neighboring sub-model bordering at the lower side of the $t^{t h}$ sub-model along the direction of $x_{i}$, the index number $\lambda_{j}^{t_{l}}$ for $j \in$ $\{1,2, \cdots, N\}$ for sub-model $t_{l}$ can be calculated from that for sub-model $t$ as

$$
\lambda_{j}^{t_{l}}=\left\{\begin{array}{l}
\lambda_{j}^{t}, j \neq i \\
\lambda_{j}^{t}-1, j=i
\end{array}\right.
$$

for $j \in\{1,2, \cdots, N\}$. According to Equation (6.11), the value of index vector $\boldsymbol{\lambda}^{t_{l}}$ for sub-model $t_{l}$ is calculated as

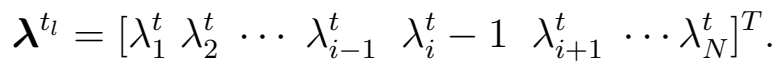


Since the $\boldsymbol{\lambda}^{t_{l}}$ and the sub-model index number $t_{l}$ has an one-to-one correspondence, we can calculate $t_{l}$ from $\boldsymbol{\lambda}^{t_{l}}$ as

$$
t_{l}=1+\sum_{j=1}^{N}\left(\left(\lambda_{j}^{t_{l}}-1\right) \cdot \prod_{p=j+1}^{N} L_{p}\right)
$$




\section{References}

[1] Y. Cao, S. Reitzinger, and Q. J. Zhang, "Simple and efficient high-dimensional parametric modeling for microwave cavity filters using modular neural network," IEEE Microw. Wireless Compon. Lett., vol. 21, no. 5, pp. 258-260, May 2011.

[2] W. Zhang, S. Yan, F. Feng, and Q. J. Zhang, "Fast and simple technique for computing circuit noise figure from component noise model using artificial neural network," in IEEE MTT-S Int. Conf. Numer. Electromagn. and Multiphys. Model. and Optim., Ottawa, ON, Canada, Aug. 2015, pp. 1-3.

[3] J. P. Garcia, F. Q. Pereira, D. C. Rebenaque, J. L. G. Tornero, and A. A. Melcon, "A neural-network method for the analysis of multilayered shielded microwave circuits," IEEE Trans. Microw. Theory Techn., vol. 54, no. 1, pp. 309-320, Jan. 2006.

[4] S. A. Sadrossadat, Y. Cao, and Q. J. Zhang, "Parametric Modeling of Microwave Passive Components Using Sensitivity-Analysis-Based Adjoint Neural-Network Technique," IEEE Trans. Microw. Theory Techn., vol. 61, no. 5, pp. 1733-1747, May 2013. 
[5] R. B. Ayed, J. Gong, S. Brisset, F. Gillon, and P. Brochet, "Three-level output space mapping strategy for electromagnetic design optimization," IEEE Trans. Magn., vol. 48, no. 2, pp. 671-674, Feb. 2012.

[6] J. E. Rayas-Sánchez, "EM-based optimization of microwave circuits using artificial neural networks: The state-of-the-art," IEEE Trans. Microw. Theory Techn., vol. 52, no. 1, pp. 420-435, Jan. 2004.

[7] S. Koziel, S. Ogurtsov, J. W. Bandler, and Q. S. Cheng, "Reliable space-mapping optimization integrated with EM-based adjoint sensitivities," IEEE Trans. Microw. Theory Tech., vol. 61, no. 10, pp. 3493-3502, Oct. 2013.

[8] J. W. Bandler, D. M. Hailu, K. Madsen, and F. Pedersen, "A space mapping interpolating surrogate algorithm for highly optimized EM-based design of microwave devices," IEEE Trans. Microw. Theory Techn., vol. 52, no. 11, pp. 2593-2600, Nov. 2004.

[9] M. B. Steer, J. W. Bandler, and C. M. Snowden, "Computer-aided design of RF and microwave circuits and systems," IEEE Trans. Microw. Theory Techn., vol. 50, no. 3, pp. 996-1005, Mar. 2002.

[10] J. Wood, D. E. Root, and N. B. Tufillaro, "A behavioral modeling approach to nonlinear model-order reduction for RF/microwave ICs and systems," IEEE Trans. Microw. Theory Techn., vol. 52, no. 9, pp. 2274-2284, Sep. 2004.

[11] F. Feng, C. Zhang, W. Na, J. Zhang, W. Zhang, and Q. J. Zhang, "Adaptive Feature Zero assisted Surrogate Based EM Optimization for Microwave Filter 
Design," IEEE Microw. Wireless Compon. Lett., vol. 29, no. 1, pp. 2-4, Jan. 2019.

[12] F. Feng, J. Zhang, W. Zhang, Z. Zhao, J. jin, and Q. J. Zhang, "Coarse- and fine-mesh space mapping for EM optimization incorporating mesh deformation," IEEE Microw. Wireless Compon. Lett., vol. 29, no. 8, pp. 510-512, Jul. 2019.

[13] A. Garcia-Lamperez, S. Llorente-Romano, M. Salazar-Palma, and T. K. Sarkar, "Efficient electromagnetic optimization of microwave filters and multiplexers using rational models," IEEE Trans. Microw. Theory Techn., vol. 52, no. 2, pp. 508-521, Feb. 2004.

[14] A. D. Huang, Z. Zhong, W. Wu, and Y. X. Guo, "An artificial neural networkbased electrothermal model for GaN HEMTs with dynamic trapping effects consideration," IEEE Trans. Microw. Theory Techn., vol. 64, no. 8, pp. 2519-2528, Aug. 2016.

[15] Z. Guo, J. Gao, Y. Cao, and Q.J. Zhang, "Passivity enforcement for passive component modeling subject to variations of geometrical parameters using neural networks," in IEEE MTT-S Int. Microw. Symp. Dig., Montreal, QC, Canada, Jun. 2012, pp. 1-3.

[16] F. Wang, V.K. Devabhaktuni, C. Xi, and Q.J. Zhang, "Neural network structures and training algorithms for microwave applications," Int. J. RF Microwave Computer-Aided Eng., vol. 9, pp. 216-240, 1999. 
[17] Q. J. Zhang, K. C. Gupta, and V. K. Devabhaktuni, "Artificial neural networks for RF and microwave design from theory to practice," IEEE Trans. Microw. Theory Techn., vol. 51, no. 4, pp. 1339-1350, Apr. 2003.

[18] J.W. Bandler, M.A. Ismail, J.E. Rayas-Sánchez, and Q.J. Zhang, "Neuromodeling of microwave circuits exploiting space-mapping technology," IEEE Trans. Microw. Theory Techn., vol. 47, no. 12, pp. 2417-2427, Dec. 1999.

[19] V.K. Devabhaktuni, B. Chattaraj, M.C.E. Yagoub, and Q.J. Zhang, "Advanced microwave modeling framework exploiting automatic model generation, knowledge neural networks, and space mapping," IEEE Trans. Microw. Theory Techn., vol. 51, no. 7, pp. 1822-1833, Jul. 2003.

[20] X. Ding, V. K. Devabhaktuni, B. Chattaraj, M. C. E. Yagoub, M. Doe, J. J. Xu, and Q. J. Zhang, "Neural network approaches to electromagnetic based modeling of passive components and their applications to high-frequency and high-speed nonlinear circuit optimization," IEEE Trans. Microw. Theory Techn., vol. 52, no. 1, pp. 436449, Jan. 2004.

[21] J. E. Rayas-Sánchez and V. Gutierrez-Ayala, "EM-based Monte Carlo analysis and yield prediction of microwave circuits using linear-input neural-output space mapping," IEEE Trans. Microw. Theory Techn., vol. 54, no. 12, pp. 4528-4537, Dec. 2006. 
[22] H. Kabir, L. Zhang, M. Yu, P. H. Aaen, J. Wood, and Q. J. Zhang, "Smart modeling of microwave device," IEEE Microw. Mag., vol. 11, no. 3, pp. 105118, May 2010.

[23] J. Xu, M. C. E. Yagoub, R. Ding, and Q. J. Zhang, "Exact adjoint sensitivity analysis for neural-based microwave modeling and design," IEEE Trans. Microw. Theory Techn., vol. 51, no. 1, pp. 226-237, Jan. 2003.

[24] S. Koziel, Q. S. Cheng, and J. W. Bandler, "Space mapping," IEEE Microwave Magazine, vol. 9, pp. 105-122, Dec. 2008.

[25] J. E. Rayas-Snchez, "Power in simplicity with ASM: tracing the aggressive space mapping algorithm over two decades of development and engineering applications," IEEE Microwave Magazine, vol. 17, no. 4, pp. 64-76, Apr. 2016.

[26] Q. S. Cheng, J. W. Bandler, and J. E. Rayas-Snchez,"Tuning-aided implicit space mapping," Int. J. RF and Microwave CAE, vol. 18, no. 5, pp. 445-453, Sep. 2008.

[27] J. L. Chvez-Hurtado, J. E. Rayas-Snchez, and Z. Brito-Brito, "Multiphysics polynomial-based surrogate modeling of microwave structures in frequency domain," in IEEE MTT-S Latin America Microwave Conf. (LAMC-2016), Puerto Vallarta, Mexico, Dec. 2016, pp. 1-3.

[28] S. Koziel, J.W. Bandler, and Q.S. Cheng, "Constrained parameter extraction for microwave design optimization using implicit space mapping," IET Microw. Antennas Propag., vol. 5, no. 10, pp. 1156-1163, Jul. 2011. 
[29] M. Sans et al., "Automated design of common-mode suppressed balanced wideband bandpass filters by means of agressive space mapping (ASM)," IEEE Trans. Microw. Theory Techn., vol. 63, no. 12, pp. 3896-3908, Dec. 2015.

[30] S. Koziel, J.W. Bandler, and Q.S. Cheng, "Tuning space mapping design framework exploiting reduced electromagnetic models," IET Microw. Antennas Propag., vol. 5, no. 10, pp. 1219-1226, Jul. 2011.

[31] F. Feng, C. Zhang, V.M.R. Gongal-Reddy, Q.J. Zhang, and J. Ma, "Parallel space-mapping approach to EM optimization," IEEE Trans. Microw. Theory Techn., vol. 62, no. 5, pp. 1135-1148, Apr. 2014.

[32] V.M.R. Gongal-Reddy, S. Zhang, C. Zhang, and Q. J. Zhang, "Parallel computational approach to gradient based EM optimization of passive microwave circuits," IEEE Trans. Microw. Theory Techn., vol. 64, no. 1, pp. 44-59, Jan. 2016.

[33] V.M.R. Gongal-Reddy, F. Feng, C. Zhang, S. Zhang, and Q. J. Zhang, "Parallel decomposition approach to gradient based EM optimization," IEEE Trans. Microw. Theory Techn., vol. 64, no. 11, pp. 3380-3399, Nov. 2016.

[34] W. Zhang, F. Feng, V.M.R. Gongal-Reddy, J. Zhang, S. Yan, J. Ma, and Q. J. Zhang, "Space mapping approach to electromagnetic centric multi-physics parametric modeling of Microwave Components," IEEE Trans. Microw. Theory Techn., vol. 66, no. 7, pp. 3169-3185, Jul. 2018. 
[35] P.H. Aaen, J. Wood, D. Bridges, L. Zhang, E. Johnson, T. Barbieri, J. Pla, C.M. Snowden, J. P. Everett, and M. J. Kearney, "Multi-physics modeling of RF and microwave high-power transistors," IEEE Trans. Microw. Theory Techn., vol. 60, no. 12, pp. 4013-4023, Dec. 2012.

[36] Y.Y. Hu, Z.R. Yu, W.J. Zhang, Q.T. Sun, and Q.H. Liu, "Multiphysics coupling of dynamic fluid flow and electromagnetic fields for subsurface sensing," IEEE J. Multiscale Multiphys. Comput. Techn., vol. 1, pp. 14-25, Apr. 2016.

[37] M.A Sanchez-Soriano et al., "Multi-physics study of RF/microwave planar devices: effect on the input signal power," in Proc. 15th Int. Conf. Threm., Mech. Multi-Phys. Simulation and Experim. Microelectron. Microsyst. (EuroSimE), Ghent, Belgium, Apr. 2014, pp. 1-7.

[38] W.Y. Yin and J.F. Mao, "Electromagnetic-thermal characterization of on-chip coupled asymmetrical interconnects," IEEE Trans. Adv. Packaging, vol. 30, no. 4, pp. 851-863, Nov. 2007.

[39] J. Sheng, Z. Jin, B. Lin, L. Ying, L. Yao, J. Zhang, Y. Li, and Z. Hong, "Electrical-thermal coupled finite element model of high temperature superconductor for resistive type fault current limiter," IEEE Trans. Appl. Supercond., vol. 22, no. 3, pp. 1-4, Jun. 2012.

[40] X. Guan, N. Shu, B. Kang, Q. Yan, Z. Li, H. Li, and X. Wu, "Multi-physics calculation and contact degradation mechanism evolution of GIB connector under daily cyclic loading," IEEE Trans. Magn., vol. 52, no. 3, pp. 1-4, Mar. 2016. 
[41] F. Feng, W. Zhang, J. Zhang, Z. Zhao, J. Jin, and Q.J. Zhang, "Recent advances in EM centric multi-physics parametric modeling," in IEEE MTT-S Int. Conf. Numerical Electromagnetic and Multiphysics Modeling and Optim., Cambridge, MA, USA, May. 2019, pp. 1-3.

[42] W. Zhang, F. Feng, S. Yan, W. Na, J. Ma, and Q. J. Zhang, "EM Centric Multiphysics Optimization of Microwave Components Using Parallel Computational Approach," IEEE Trans. Microw. Theory Techn., early access, Nov. 2019.

[43] F. Wang and Q. J. Zhang, "Knowledge based neural models for microwave design," IEEE Trans. Microw. Theory Techn., vol. 45, no. 12, pp. 2333-2343, Dec. 1997.

[44] V. B. Litovski, J. I. Radjenovic, Z. M. Mrcarica, and S. L. Milenkovic, "MOS transistor modeling using neural network," Elect. Lett., vol. 28, no. 18, pp. 17661768, Aug. 1992.

[45] V. K. Devabhaktuni, C. Xi, and Q. J. Zhang, "A neural network approach to the modeling of heterojunction bipolar transistors from S-parameter data," Proc. $28^{\text {th }}$ European Microw. Conf., Amsterdam, Netherlands, Oct. 1998, pp. 306-311.

[46] M. Vai and S. Prasad, "Qualitative modeling heterojunction bipolar transistors for optimization: A neural network approach," Proc. IEEE/Cornell Conf. Adv. Concepts in High Speed Semiconductor Dev. and Circuits, pp. 219-227, Aug. 1993. 
[47] K. Shirakawa, M. Shimizu, N. Okubo, and Y. Daido, "Structural determination of multilayered large signal neural-network HEMT model," IEEE Trans. Microw. Theory Techn., vol. 46, no. 10, pp. 1367-1375, Oct. 1998.

[48] A. H. Zaabab, Q. J. Zhang, and M. S. Nakhla, "A neural network modeling approach to circuit optimization and statistical design," IEEE Trans. Microw. Theory Techn., vol. 43, no. 6, pp. 1349-1358, June 1995.

[49] Y. Fang, M. C. E. Yagoub, F. Wang, and Q. J. Zhang, "A new macromodeling approach for nonlinear microwave circuits based on recurrent neural networks," IEEE Trans. Microw. Theory Techn., vol. 48, no. 12, pp. 2335-2344, Dec. 2000.

[50] A. Veluswami, M. S. Nakhla, and Q. J. Zhang, "The application of neural networks to EM-based simulation and optimization of interconnects in highspeed VLSI circuits," IEEE Trans. Microw. Theory Techn., vol. 45, no. 5, pp. 712-723, May 1997.

[51] Q. J. Zhang and M. S. Nakhla, "Signal integrity analysis and optimization of VLSI interconnects using neural network models," IEEE Int. Circuits Syst. Symp., London, England, May 1994, pp. 459-462.

[52] Y. Cao, L. Simonovich, and Q. J. Zhang, "A broadband and parametric model of differential via holes using space-mapping neural network," IEEE Microw. Wireless Compon. Lett., vol. 19, no. 9, pp. 533-535, Sep. 2009.

[53] X. Ding, J. Xu, M. C. E. Yagoub, and Q. J. Zhang, "A combined state space formulation/equivalent circuit and neural network technique for modeling of em- 
bedded passives in multilayer printed circuits," J. of the Applied Computational Electromagnetics Society, vol. 18, 2003.

[54] C. Christodoulou, A. E. Zooghby, and M. Georgiopoulos, "Neural network processing for adaptive array antennas," IEEE-APS Int. Symp., Orlando, FL, July 1999, pp.2584-2587.

[55] V. M. R. Gongal-Reddy, F. Feng, and Q. J. Zhang, "Parametric modeling of millimeter-wave passive components using combined neural networks and transfer functions," in Global Symposium On Millimeter Waves (GSMM), Montreal, QC, Canada, May 2015, pp. 1-3.

[56] P. M. Watson and K. C. Gupta, "Design and optimization of CPW circuits using EM-ANN models for CPW components," IEEE Trans. Microw. Theory Techn., vol. 45, no. 12, pp. 2515-2523, Dec. 1997.

[57] M. H. Bakr, J. W. Bandler, M. A. Ismail, J. E. Rayas-Sánchez, and Q. J. Zhang, "Neural space mapping EM optimization of microwave structures," IEEE MTTS Int. Microw. Symp. Dig., Boston, MA, Jun. 2000, pp. 879-882.

[58] H. Sharma and Q. J. Zhang, "Transient electromagnetic modeling using recurrent neural networks," IEEE MTT-S Int. Microw. Symp. Dig., San Francisco, CA, Jun. 2005, pp. 1597-1600.

[59] H. Kabir, Y. Wang, M. Yu, and Q. J. Zhang, "Neural network inverse modeling and applications to microwave filter design," IEEE Trans. Microw. Theory Techn., vol. 56, no. 4, pp. 867-879, Apr. 2008. 
[60] W. Zhang, F. Feng, J. Zhang, Z. Zhao, J. Ma, and Q. J. Zhang, "Parallel decomposition approach for wide range parametric modeling of microwave components," IEEE Trans. Microw. Theory Techn, submitted, Jan. 2020.

[61] Q. J. Zhang, G. Wilson, R. Venkatachalam, A. Sarangan, J. Williamson, and F. Wang, "Ultra fast neural models for analysis of electro/optical interconnects," Proc. IEEE Electronic Components and Techn. Conf. San Jose, CA, May 1994, pp. 1134-1137.

[62] G. Fedi, S. Manetti, G. Pelosi, and S. Selleri, "Design of cylindrical posts in rectangular waveguide by neural network approach," IEEE AP-S Int. Symp. Digest, Salt Lake City, UT, Jul. 2000, pp. 1054-1057.

[63] V. Rizzoli, A. Neri, D. Masotti, and A. Lipparini, "A new family of neural network-based bidirectional and dispersive behavioral models for nonlinear RF/microwave subsystems," Int. J. RF Microw. Comput.- Aided Eng., vol. 12, no. 1, pp. 51-70, Jan. 2002.

[64] J. Xu, M. C. E. Yagoub, R. Ding, and Q. J. Zhang, "Neural based dynamic modeling of nonlinear microwave circuits," IEEE Trans. Microw. Theory Techn., vol. 50, no. 12, pp. 2769-2780, Dec. 2002.

[65] Y. Cao, J. J. Xu, V. K. Devabhaktuni, R. T. Ding, and Q. J. Zhang, "An adjoint dynamic neural network technique for exact sensitivities in nonlinear transient modeling and high-speed interconnect design," in IEEE MTT-S Int. Microw. Symp. Dig., PA, Philadelphia, Jun. 2003, pp. 165-168. 
[66] T. Liu, S. Boumaiza, and F. M. Ghannouchi, "Dynamic behavioral modeling of 3G power amplifiers using real-valued time-delay neural networks," IEEE Trans. Microw. Theory Techn., vol. 52, no. 3, pp. 1025-1033, Mar. 2004.

[67] J. Jin, C. Zhang, F. Feng, W. Na, J. Ma and Q. J. Zhang, "Deep neural network technique for high-dimensional microwave modeling and applications to parameter extraction of microwave filters," IEEE Trans. Microw. Theory Techn., vol. 67, no. 10, pp. 4140 - 4155, Aug. 2019.

[68] G. Cybenko, "Approximation by superpositions of a sigmoidal function," Math. Control Signals Systems, vol. 2, pp. 303-314, 1989.

[69] K. Hornik, M. Stinchcombe, and H. White, "Multilayer feedforward networks are universal approximators," Neural Networks, vol. 2, pp. 359-366, 1989.

[70] T. Y. Kwok, and D. Y. Yeung, "Constructive algorithms for structure learning in feedforward neural networks for regression problems," IEEE Trans. Neural Networks, vol. 8, no. 3, pp. 630-645, May 1997.

[71] R. Reed, "Pruning algorithms - a Survey," IEEE Trans. Neural Networks, vol. 4, no. 5, pp. 740-747, Sep. 1993.

[72] A. Krzyzak, and T. Linder, "Radial basis function networks and complexity regularization in function learning," IEEE Trans. Neural Networks, vol. 9, no. 2, pp. 247-256, Mar. 1998. 
[73] W. Na and Q. J. Zhang, "Automated knowledge-based neural network modeling for microwave applications," IEEE Microw. Wireless Compon. Lett., vol. 24, no. 7, pp. 499-501, Jul. 2014.

[74] J. de Villiers and E. Barnard, "Backpropagation neural nets with one and two hidden layers," IEEE Trans. Neural Networks, vol. 4, no. 1, pp. 136-141, Jan. 1993.

[75] S. Tamura, and M. Tateishi, "Capabilities of a four-layered feedforward neural network: four layer versus three," IEEE Trans. Neural Networks, vol. 8, no. 2, pp. 251-255, Mar. 1997.

[76] I. S. Stievano, I. A. Maio, and F. G. Canavero, "Parametric macromodels of digital I/O ports," IEEE Trans. Adv. Packag., vol. 25, no. 5, pp. 255-264, May 2002.

[77] J. A. Garcia, et al., "Modeling MESFET's and HEMT's intermodulation distortion behavior using a generalized radial basis function network," Int. Journal of RF and Microwave CAE, Special Issue on Applications of ANN to RF and Microwave Design, Vol. 9, pp. 261-276, 1999.

[78] Q. H. Zhang, "Using wavelet network in nonparametric estimation," IEEE Trans. Neural Networks, vol. 8, no. 2, pp. 227-236, Mar. 1997.

[79] Q. H. Zhang and A. Benvensite, "Wavelet networks," IEEE Trans. Neural Networks, vol. 3, no. 6, pp. 889-898, Nov. 1992. 
[80] Y. C. Pati and P. S. Krishnaprasad, "Analysis and synthesis of feedforward neural networks using discrete affine wavelet transformations," IEEE Trans. Neural Networks, vol. 4, no. 1, pp. 73-85, Jan. 1993.

[81] J. Aweya, Q. J. Zhang, and D. Montuno, "A direct adaptive neural controller for flow control in computer networks," IEEE Int. Conf. Neural Networks, Anchorage, Alaska, pp. 140-145, May 1998.

[82] J. Aweya, Q. J. Zhang, and D. Montuno, "Modelling and control of dynamic queues in computer networks using neural networks," IASTED Int. Conf. Intelligent Syst. Control, Halifax, Canada, pp. 144-151, June 1998.

[83] L. H. Tsoukalas, and R. E. Uhrig, Fuzzy and Neural Approaches in Engineering, NY: Wiley-Interscience, 1997.

[84] J. Zhang, C. Zhang, F. Feng, W. Zhang, J. Ma, and Q. J. Zhang, "Polynomial chaos-based approach to yield-driven EM optimization," IEEE Trans. Microw. Theory Techn., vol. 66, no. 7, pp. 3186-3199, Jul. 2018.

[85] Y. Cao, G. Wang, and Q. J. Zhang, "A new training approach for parametric modeling of microwave passive components using combined neural networks and transfer functions," IEEE Trans. Microw. Theory Techn., vol. 57, no. 11, pp. 2727-2742, Nov. 2009.

[86] F. Feng, C. Zhang, J. Ma, and Q. J. Zhang, "Parametric modeling of EM behavior of microwave components using combined neural networks and pole- 
residue-based transfer functions," IEEE Trans. Microw. Theory Techn., vol. 64, no. 1, pp. 60-77, Jan. 2016.

[87] B. Gustavsen and A. Semlyen, "Rational approximation of frequency domain responses by vector fitting," IEEE Trans. Power Del., vol. 14, no. 3, pp. 10521061, Jul. 1999.

$\mathrm{lk}$

[88] J. W. Bandler, Q. S. Cheng, N. K. Nikolova, and M. A. Ismail, "Implicit space mapping optimization exploiting preassigned parameters," IEEE Trans. Microw. Theory Tech., vol. 52, no. 1, pp. 378-385, Jan. 2004.

[89] J. W. Bandler, M. A. Ismail, and J. E. Rayas-Sánchez, "Expanded spacemapping EM-based design framework exploiting preassigned parameters," IEEE Trans. Circuits Syst. I, Fundam. Theory Appl., vol. 49, no. 12, pp. 1833-1838, Dec. 2002.

[90] S. Koziel, J. W. Bandler, and K. Madsen, "Space mapping with adaptive response correction for microwave design optimization," IEEE Trans. Microw. Theory Techn., vol. 57, no. 2, pp. 478-486, Feb. 2009.

[91] L. Zhang, J. Xu, M. C. E. Yagoub, R. Ding, and Q. J. Zhang, "Efficient analytical formulation and sensitivity analysis of neuro-space mapping for nonlinear microwave device modeling," IEEE Trans. Microw. Theory Techn., vol. 53, no. 9, pp. 2752-2767, Sep. 2005. 
[92] M. H. Bakr, J. W. Bandler, M. A. Ismail, J. E. Rayas-Sánchez, and Q. J. Zhang, "Neural space-mapping optimization for EM-based design," IEEE Trans. Microw. Theory Techn., vol. 48, no. 12, pp. 2307-2315, Dec. 2000.

[93] V. Gutierrez-Ayala and J. E. Rayas-Sánchez, "Neural input space mapping optimization based on nonlinear two-layer perceptrons with optimized nonlinearity," Int. J. RF Microw. Comput.-Aided Eng., vol. 20, no. 5, pp. 512-526, Sep. 2010.

[94] F. Feng and Q. J. Zhang, "Neural space mapping optimization for EM design," in Proc. Asia-Pacific Microw. Conf., Nanjing, China, Dec. 2015, pp. 1-3.

[95] D. Gorissen, L. Zhang, Q. J. Zhang, and T. Dhaene, "Evolutionary neuro-space mapping technique for modeling of nonlinear microwave devices," IEEE Trans. Microw. Theory Techn., vol. 59, no. 2, pp. 213-229, Feb. 2011.

[96] S. Koziel, J. W. Bandler, and K. Madsen, "A space mapping framework for engineering optimization: theory and implementation," IEEE Trans. Microw. Theory Techn., vol. 54, no. 10, pp. 3721-3730, Oct. 2006.

[97] J. Meng, S. Koziel, J. W. Bandler, M. H. Bakr, and Q. S. Cheng, "Tuning space mapping: A novel technique for engineering design optimization," in IEEE MTT-S Int. Microw. Symp. Dig.,, Atlanta, Georgia, Jun. 2008, pp. 991-994.

[98] L. Zhang, P. H. Aaen, and J. Wood, "Portable space mapping for efficient statistical modeling of passive components," IEEE Trans. Microw. Theory Techn., vol. 60, no. 3, pp. 441-450, Mar. 2012. 
[99] C. Zhang, F. Feng, and Q. J. Zhang, "EM optimization using coarse and fine mesh space mapping," in Proc. Asia-Pacific Microw. Conf., Seoul, Korea, Dec. 2013, pp. 824-826.

[100] F. Feng, C. Zhang, V. M. R. Gongal-Reddy, and Q. J. Zhang, "Knowledgebased coarse and fine mesh space mapping approach to EM optimization," in Int. Conf. Numerical Electromagnetic Modeling and Optimization, Pavia, Italy, May. 2014, pp. 1-4.

[101] S. Koziel, Q. S. Cheng, and J. W. Bandler, "Fast EM modeling exploiting shape-preserving response prediction and space mapping," IEEE Trans. Microw. Theory Tech., vol. 62, no. 3, pp. 399-407, Mar. 2014.

[102] J. W. Bandler, Q. S. Cheng, S. A. Dakroury, A. S. Mohamed, M. H. Bakr, K. Madsen, and J. Sondergaard, "Space mapping: The state of the art," IEEE Trans. Microw. Theory and Techn., vol. 52, no. 1, pp. 337-361, Jan. 2004.

[103] J. W. Bandler, Q. S. Cheng, D. H. Gebre-Mariam, K. Madsen, F. Pedersen, and J. Sondergaard, "EM-based surrogate modeling and design exploiting implicit, frequency and output space mappings," in IEEE MTT-S Int. Microwave Symp. Dig., Philadelphia, PA, Jun. 2003, pp. 1003-1006

[104] N. M. Alexandrov, J. E. Dennis Jr., R. M. Lewis, and V. Torczon, "A trustregion framework for managing the use of approximation models in optimizations," Struct. Optim., vol. 15, no. 1, pp. 16-23, Feb. 1998. 
[105] N. M. Alexandrov and R. M. Lewis, "An overview of first-order model management for engineering optimization," Optim. Eng., vol. 2, no. 4, pp. 413-430, Dec. 2001.

[106] N. M. Alexandrov, R. M. Lewis, C. R. Gumbert, L. L. Green, and P. A. Newman, "Approximation and model management in aerodynamic optimization with variable-fidelity models," J. of Aircraft, vol. 38, no. 6, pp. 1093-1101, Dec. 2001

[107] R. Trobec, M. Vajtersic, and P. Zinterhof, Parallel Computing: Numerics, Applications, and Trends, Springer Dordrecht Heidelberg London New York, 2009.

[108] L. Zhang, Y. Cao, S. Wan, H. Kabir, and Q. J. Zhang, "Parallel automatic model generation technique for microwave modeling," in IEEE MTT-S Int. Microw. Symp. Dig., Honolulu, HI, Jun. 2007, pp. 103-106.

[109] F. Feng, V. M. R. Gongal-Reddy, C. Zhang, W. Na, S. Zhang, and Q. J. Zhang, "Recent advances in parallel EM optimization approaches," in IEEE MTT-S Int. Conf. Microw. Millimeter Wave Technology, Beijing, China, Jun. 2016, pp. 1-3.

[110] F. Feng, C. Zhang, S. Zhang, V. M. R. Gongal-Reddy, and Q. J. Zhang, "Parallel EM optimization approach to microwave filter design using feature assisted neuro-transfer functions," in IEEE MTT-S Int. Microw. Symp. Dig., San Francisco, CA, May. 2016, pp. 1-3. 
[111] J. Zhang, K. Ma, F. Feng, Z. Zhao, W. Zhang, and Q. J. Zhang, "Distributed parallel computing technique for EM modeling," in IEEE MTT-S Int. Conf. Numerical Electromagnetic and Multiphysics Modeling and Optim., Ottawa, Canada, Aug. 2015, pp. 1-3.

[112] J. Zhang, K. Ma, F. Feng, and Q. J. Zhang, "Parallel gradient-based local search accelerating particle swarm optimization for training microwave neural network models," in IEEE MTT-S Int. Microw. Symp. Dig., Phoenix, AZ, May. 2015, pp. 1-3.

[113] V. M. R. Gongal-Reddy, S. Zhang, Y. Cao, and Q. J. Zhang, "Efficient design optimization of microwave circuits using parallel computational methods," in Proc. Yth Eur. Microw. Integr. Circuits Conf., Amsterdam, The Netherlands, Oct. 2012, pp. 254-257.

[114] S. Koziel and J. W. Bandler, "Space mapping with distributed fine model evaluation for optimization of microwave structures and devices," in IEEE MTTS Int. Microw. Symp. Dig., Atlanta, GA, Jun. 2008, pp. 1377- 1380.

[115] S. Koziel and J. W. Bandler, "Distributed fine model evaluation for rapid space-mapping optimisation of microwave structures," IET Microw. Antennas Propag., vol. 3, no. 5, pp. 798-807, Aug. 2009.

[116] W. Zhang, F. Feng, J. Zhang, S. Zhang, V. M. R. Gongal-Reddy, and Q. J. Zhang, "Advanced parametric modeling using neuro-transfer function for EM 
based multiphysics analysis of microwave passive components," in IEEE MTT-S Int. Microw. Symp. Dig., San Francisco, CA, USA, May. 2016, pp. 1-3.

[117] S. Yan, Y. Zhang, X. Jin, W. Zhang, and W. Shi, "Multi-physics parametric modeling of microwave passive components using artificial neural networks," Prog. Electromagn. Res. M, vol. 72, pp. 79-88, Aug. 2018.

[118] W. Na, W. Zhang, S. Yan, F. Feng, W. Zhang, and Y. Zhang, "Automated neural network-based multiphysics parametric modeling of microwave components," IEEE Access, vol. 7, pp. 141153 - 141160, Sep. 2019.

[119] Z. Huang and J. Fang, "Multiphysics design and optimization of high-speed permanent-magnet electrical machines for air blower applications," IEEE Trans. Ind. Electron., vol. 63, no. 5, pp. 2766-2774, May 2016.

[120] S. Sirci, M. A: S. Soriano, and D. Jorge "Design and multiphysics analysis of direct and cross-coupled siw combline filters using electric and magnetic couplings," IEEE Trans. Microw. Theory Tech., vol. 63, no.12, pp.4341-4354, Dec. 2015 .

[121] F. Wu, "Solution of large-scale networks by tearing," IEEE Trans. Circuits Syst., vol. 23, no. 12, pp. 706-713, Dec. 1976.

[122] S. Liao, H. Kabir, Y. Cao, J. Xu, Q. J. Zhang, and J. Ma, "Neural-network modeling for 3-D substructures based on spatial EM-field cou-pling in finiteelement method," IEEE Trans. Microw. Theory Techn., vol. 59, no. 1, pp. 21-38, Jan. 2011. 
[123] H. Kabir, Y. Wang, M. Yu, and Q. J. Zhang, "High-dimensional neuralnetwork technique and applications to microwave filter modeling," IEEE Trans. Microw. Theory Techn., vol. 58, no. 1, pp. 145-156, Jan. 2010.

[124] J. W. Bandler and Q. J. Zhang, "An automatic decomposition approach to optimization of large microwave systems," IEEE Trans. Microw. Theory Techn., vol. 35 , no. 12 , pp. 12311239 , Dec. 1987

[125] S. R. Schmidt and R. G. Launsby, Understanding industrial designed experiments. Colorado Springs, CO: Air Force Academy, 1992.

[126] F. Feng and C. Zhang, J. Ma, and Q. J. Zhang, "Parametric modeling of microwave components using adjoint neural networks and pole-residue transfer functions with EM sensitivity analysis," IEEE Trans. Microw. Theory Techn., vol. 65, no. 6, pp. 1955-1975, Jun. 2017.

[127] C. Zhang, F. Feng, Q. J. Zhang, and J. W. Bandler, "Cognition-driven formulation of space mapping for equal-ripple optimization of microwave filters," IEEE Trans. Microw. Theory Techn., vol. 63, no. 7, pp. 2154-2165, Jul. 2015.

[128] X. Liu, L. P. B. Katehi, W. J. Chappell, and D. Peroulis, "Power handling of electrostatic MEMS evanescent-mode (EVA) tunable bandpass filters," IEEE Trans. Microw. Theory Techn., vol. 60, no. 2, pp. 270-283, Feb. 2012.

[129] V. K. Devabhaktuni, M. C. E. Yagoub, and Q. J. Zhang, "A robust algorithm for automatic development of neural-network models for microwave appli- 
cations," IEEE Trans. Microw. Theory Techn., vol. 49, no. 12, pp. 2282-2291, Dec. 2001.

[130] W. Na, and Q. J. Zhang, "Automated parametric modeling of microwave components using combined neural network and interpolation techniques," in IEEE MTT-S Int. Microw. Symp. Dig., Seattle, WA, USA, Jun. 2013, pp. 1-3.

[131] M. A. E. Sabbagh, M. H. Bakr and J. W. Bandler, "Adjoint higher order sensitivities for fast full-wave optimization of microwave filters," IEEE Trans. Microw. Theory Techn., vol. 54, no. 8, pp. 3339-3351, Aug. 2006. 